\title{
Nuclear spin physics in quantum dots: an optical investigation
}

\author{
Bernhard Urbaszek, Xavier Marie and Thierry Amand \\ Université de Toulouse, INSA-CNRS-UPS, LPCNO, 135 Av. Rangueil, 31077 Toulouse, France \\ Olivier Krebs and Paul Voisin \\ Laboratoire de Photonique et Nanostructures CNRS, route de Nozay, 91460 Marcoussis, France \\ Patrick Maletinsky \\ Department of Physics, University of Basel, Klingelbergstrasse 82, Basel CH-4056, Switzerland
}

Alexander Högele

Ludwig-Maximilians-Universität, Fakultät für Physik and CeNS, Geschwister-Scholl-Platz 1, D-80539 München, Germany

Atac Imamoglu

Institute of Quantum Electronics, ETH-Zurich, CH-8093, Zurich, Switzerland

\begin{abstract}
The mesoscopic spin system formed by the $10^{4}-10^{6}$ nuclear spins in a semiconductor quantum dot offers a unique setting for the study of many-body spin physics in the condensed matter. The dynamics of this system and its coupling to electron spins is fundamentally different from its bulk counter-part as well as that of atoms due to increased fluctuations that result from reduced dimensions. In recent years, the interest in studying quantum dot nuclear spin systems and their coupling to confined electron spins has been fueled by its direct implication for possible applications of such systems in quantum information processing as well as by the fascinating nonlinear (quantum-)dynamics of the coupled electron-nuclear spin system. In this article, we review experimental work performed over the last decades in studying this mesoscopic, coupled electron-nuclear spin system and discuss how optical addressing of electron spins can be exploited to manipulate and read-out quantum dot nuclei. We discuss how such techniques have been applied in quantum dots to efficiently establish a non-zero mean nuclear spin polarization and, most recently, were used to reduce fluctuations of the average quantum dot nuclear spin orientation. Both results in turn have important implications for the preservation of electron spin coherence in quantum dots, which we discuss. We conclude by speculating how this recently gained understanding of the quantum dot nuclear spin system could in the future enable experimental observation of quantummechanical signatures or possible collective behavior of mesoscopic nuclear spin ensembles.
\end{abstract}

\section{Contents}

\section{INTRODUCTION}

II. BASICS OF SEMICONDUCTOR QUANTUM DOTS

A. Growth and sample structures

B. Addressing individual charge states

C. Electronic states, Optical selection rules and Carrier Coulomb exchange interactions

D. Optical spectroscopy techniques

E. Electron spin orientation mechanisms

III. ELEMENTARY INTERACTIONS WITH NUCLEI IN QUANTUM DOTS

A. Magnetic coupling of electrons to nuclei: Hyperfine interaction

B. Electrostatic coupling: Nuclear quadrupole effects

IV. DYNAMICS OF ELECTRON SPINS COUPLED TO A FLUCTUATING NUCLEAR FIELD

A. The Merkulov-Efros-Rosen Model

B. Experimental studies of electron spin dephasing in zero external magnetic field

C. Electron spin dephasing in a longitudinal magnetic field: Faraday geometry

D. Electron spin dephasing in a transverse magnetic field: Voigt geometry
E. Influence of the fluctuating nuclear field on electron and hole spin pumping processes

F. Beyond the nuclear mean field approach

V. OPTICAL PUMPING OF NUCLEAR SPINS

A. Dynamic Nuclear Polarization : general features 21

B. Dynamic nuclear polarization in single quantum dot optics

1. Nonlinearity of the dynamic nuclear polarization 23

2. Dynamic nuclear polarization in the presence of nuclear quadrupole effects

3. Nuclear field bistability in magnetic field sweeps 25

4. Nonlinearity of the nuclear polarization as a function of excitation laser polarization

5. Overhauser effect in zero magnetic field : role of the Knight field

6. Nuclear field versus electron-hole exchange : the case of neutral excitons

C. Nuclear spin polarization under resonant optical excitation

1. Overhauser effects under resonant optical excitation

2. Pumping of Nuclear Spins by Optical Excitation of Spin-Forbidden Transitions

3. Locking of quantum-dot resonances to an incident laser

4. Preparation of nuclear spin states using periodic pulsed-laser excitation of quantum dot ensembles 39 


\section{NUCLEAR SPIN DYNAMICS IN QUANTUM} DOTS

A. Dynamics of nuclear spin polarization in low magnetic fields

1. Nuclear spin relaxation in semiconductor quantum dots

B. Nonlinear nuclear spin dynamics in high magnetic fields

C. Nuclear Spin dynamics at milliKelvin temperatures 45

D. Dynamic nuclear polarization in a transverse field: the anomalous Hanle effect

E. Optically detected NMR on semiconductor quantum dots

F. Irreversibility and hysteresis in demagnetization experiments

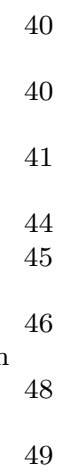

VII. HOLE SPINS COUPLED TO NUCLEAR SPINS51

A. Hole spin dephasing due to hyperfine interaction

B. Overhauser effect for holes

\section{PERSPECTIVES}

Acknowledgments

References

\section{INTRODUCTION}

Electronic spins in most semiconductors are relatively well decoupled from orbital or charge degrees of freedom. As a consequence, electronic spin coherence is not hindered by the prevalent charge decoherence, rendering spins good candidates for the realization of novel devices whose functionalities rely on quantum coherence. The isolation of spins from adverse effects of fluctuating charge environments is particularly effective in quantum dots (QDs) where electronic motion is quantum confined in all directions to length scales on the order of 10 nanometers. In such atom-like structures, hyperfine coupling is the dominant interaction for both the spin of the electron confined to the QD and the nuclear spins, making this system a nearly ideal realization of the central spin model.

In this Article, we review recent work literally shedding light on this unique coupled spin system. The basic principle of optical manipulation and measurement of QD spins we describe has its roots in the use of strong spin-orbit interaction of valence band states which allow for correlating the optically excited electron spin with the polarization of the excitation laser. This is in fact the same physics used in optical pumping experiments carried out in atomic vapors: in 1952 Kastler, Brossel and Winter investigated Mercury atoms in a weak magnetic field which splits the electron states into Zeeman sublevels. By irradiation of the atoms with circularly polarized light the authors could selectively populate one of the electron Zeeman levels (Brossel et al., 1952). Subsequent optical pumping experiments on atoms with nonzero nuclear spin resulted in direct preparation of correlated electron-nuclear spin states in atoms.

In pioneering work in the solid-state, Knight observed that polarized electrons lead to a shift in nuclear mag-

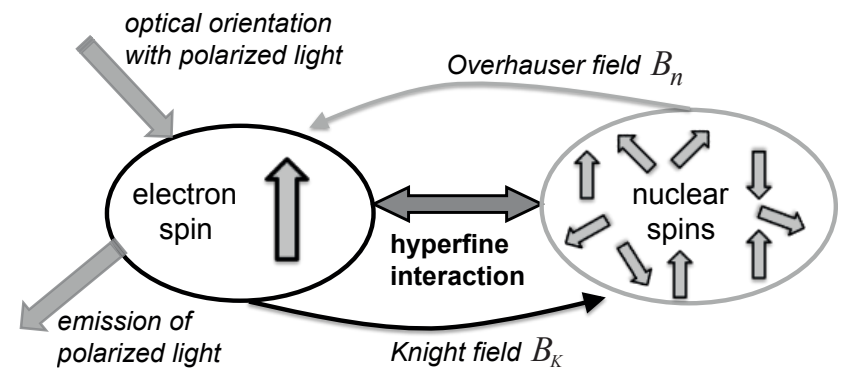

FIG. 1 Reciprocal interaction between electron and nuclear spins. The electron spin state is initialised through optical pumping.

netic resonance frequency (Knight, 1949). In 1953 Overhauser proposed to polarize nuclear spins by transferring spin polarization from electrons to the nuclear spin system. In this original proposal, a net electron spin polarization was created simply by allowing thermalisation in an applied longitudinal field (Overhauser, 1953). Soon afterwards the ideas of Overhauser and Brossel et $a l$ were combined in the first work on optical preparation of electron spins and the resulting interaction of these spin polarized electrons with the nuclear spin system in a semiconductor (Lampel, 1968). In this experiment performed with silicon the initial pumping of spin oriented conduction electrons induced by polarized light leads to a polarization of the nuclear spins of the atoms of the silicon lattice via the hyperfine interaction (Overhauser effect). This is based on the angular momentum transfer between photons and electrons and subsequently between electrons and nuclei. The nuclear polarization was detected by Lampel through the enhancement of the nuclear magnetic resonance signal. A detailed review of the nuclear spin effects in bulk semiconductor optics can be found in Meier and Zakharchenya (1984), where the key ingredients for strong hyperfine effects in solids were clearly identified: localization of the carrier wave function around a finite number of nuclei and temporal fluctuations in the electron spin system i.e. a short correlation time of the hyperfine interaction. As a result strong nuclear effects imprinted on the polarization of the emitted photons were observed in n-doped bulk semiconductors which show strong localisation of carriers around donors (Dzhioev et al., 2002).

Due to the strong localization of the carrier wave function in a $\mathrm{QD}$, the role of hyperfine interactions in spin dynamics is drastically enhanced; this is a direct consequence of enhanced fluctuations in the effective magnetic field seen by the electron spin (Overhauser field) due to its interactions with randomly oriented QD nuclei. Similarly, the effective magnetic field seen by each nucleus (Knight field) is more susceptible to fluctuations in the electron spin. Soon after the first observation of emission from single QDs (Marzin et al., 1994) it became clear that studies of the electron spin system cannot be done without taking nuclear effects into account. Conversely, ultra- 
narrow QD optical transition linewidths allow for a direct measurement of the nuclear field, greatly enhancing the possibilities for investigating nuclear spin dynamics using optical spectroscopy. This is shown in pioneering work on optically detected nuclear magnetic resonance ODNMR in GaAs dots in AlGaAs (Brown et al. 1998, Gammon et al. 1997).

Initialization of an individual electron or hole spin with a laser pulse is possible due to angular momentum transfer between photons and electrons, enabled by spin-orbit interaction and ensuing optical selection rules. Once initialized, the prospects for controlled, coherent manipulations of spins in QDs are very good as the main spin relaxation mechanisms known from experiments in bulk or 2D semiconductors do not apply to localized carriers in dots (Khaetskii and Nazarov, 2000, Kroutvar et al. 2004, Paillard et al., 2001, Pines et al., 1957). However, it had been pointed out early (Burkard et al., 1999, Dyakonov and Perel., 1973, 1974, Merkulov et al., 2002) that interactions with fluctuating, arbitrarily aligned nuclear spins of the atoms that form the QD might severely limit the electron spin coherence time. This prediction has indeed been confirmed independently for electrons in transport measurements and in optical spectroscopy (Braun et al. 2005, Petta et al. 2005). Extending the carrier spin coherence time for controlled quantum state (qubit) manipulation was one of the strong motivations that led to increased interest in nuclear spin physics in QDs (Bluhm et al. 2010). Many fascinating experiments have been reported confirming the strong, reciprocal interaction between the spin systems. For example, the magnitude and direction of the Overhauser field created via optical pumping can be tuned by adjusting laser power and polarization (Bracker et al., 2005, Eble et al. 2006, Maletinsky et al. 2009, Tartakovskii et al., 2007). The nuclear spin system can be stable up to several hours under certain conditions, which is interesting for information storage schemes (Taylor et al. 2003). The hyperfine interaction allows for tuning the exact energy of the electronic states and for controlling the polarization of the emitted light. This is particularly true for experiments in the absence of magnetic fields (Belhadj et al. 2009 , Lai et al. 2006, Larsson et al., 2011) and could become important for applications in photonics. For example, knowing the exact polarization basis is crucial when evaluating the degree of entanglement of a source of photon pairs based on optical transitions from the conduction to valence state in a single quantum dot (Akopian et al. 2006, Dousse et al., 2010, Stevenson et al., 2011).

Hyperfine effects in QDs can have other spectacular consequences, such as locking of a QD transition to a resonant pump laser (Chekhovich et al., 2010a; Latta et al. 2009, Xu et al., 2009), bistability of the nuclear spin system (Braun et al. , 2006b; Kaji et al., 2008, Maletinsky et al. 2007b; Tartakovskii et al., 2007) depending not only on the experimental parameters at the time the measurement was performed but also on the history of the experiment (non-Markovian behaviour). The meso- (a)

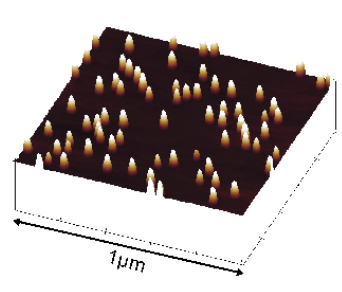

(b)

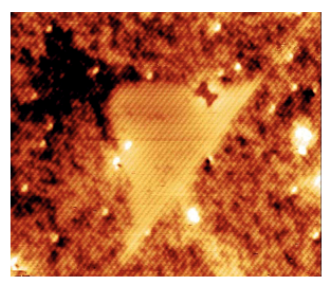

(c)

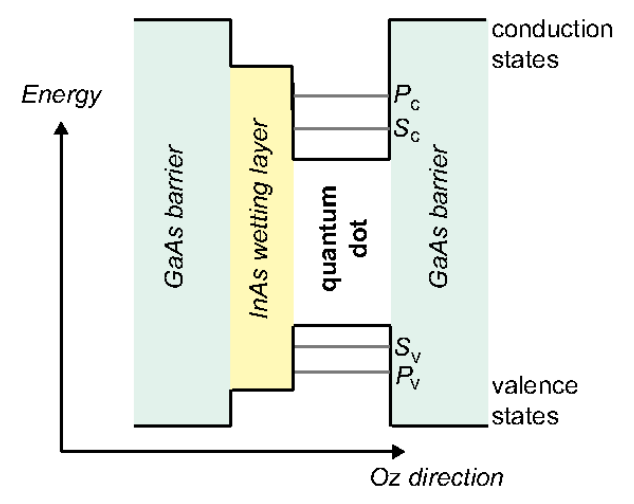

FIG. 2 (a) $1 \mu \mathrm{m} \times 1 \mu \mathrm{m}$ Atomic force microscopy image of InAs dots on GaAs (b) $40 \mathrm{~nm} \times 34 \mathrm{~nm}$ cross-sectional scanning tunneling microscopy image of a GaAs dot in AlGaAs (Keizer et al. 2010). (c) schematic energy level diagram for an InAs QD in GaAs, where the growth axis is along the $O z$ direction.

scopic nuclear spin system may enable observation of physical phenomena such as Levy flights (Issler et al. 2010), spin-squeezed states (Rudner et al., 2011) and dissipative quantum phase transitions (Kessler et al., 2012, 2010). Studies of hyperfine effects in dots are also relevant for other systems with localised carriers, such as nitrogen vacancy centres in diamond Balasubramanian et al. 2009, Childress et al. 2006).

\section{BASICS OF SEMICONDUCTOR QUANTUM DOTS}

Semiconductor QDs are nanometer sized objects that contain typically several thousand atoms of a semiconducting compound resulting in a quantum confinement of the carriers in the three spatial directions. As a consequence, the energy levels in semiconductor QDs are discrete. Micro-photoluminescence experiments (Marzin et al. 1994), photon correlation measurements (Michler et al., 2000) and resonant laser scattering (Högele et al. , 2004) have established the atom like character of the interband transitions. This motivated many research groups to probe and manipulate charge and spin states of individual carriers. These experiments test the possibility of using these QD states as qubits for quantum information processing (Henneberger and Benson, 2008). 


\section{A. Growth and sample structures}

Semiconductor QDs can be synthesized by a large variety of methods based on colloidal chemistry, molecular beam epitaxy (MBE) or metalorganic chemical vapor deposition (MOCVD). QDs can be formed at interface steps of thin quantum wells (Besombes et al., 2000, Gammon et al. 1997, 1996; Hours et al. , 2005) or by self assembly in the Stransky-Krastanov growth mode during molecular beam epitaxy (Goldstein et al., 1985, Leonard et al. 1994). The latter process is driven by the strain resulting from the smaller lattice parameter of the matrix (barrier) compared to that of the dots, for example $7 \%$ for InAs dots in GaAs. The QDs obtained in this well studied system are typically $20 \mathrm{~nm}$ in diameter and $5 \mathrm{~nm}$ in height (see Fig.2(a)) and are formed on a thin InAs quantum well called wetting layer, as can be seen in STM measurements (Offermans et al., 2005). Samples used for optical spectroscopy are then covered again by the barrier material. In realistic samples InAs dots contain a significant fraction $x$ of Ga, leading to the formation of $\mathrm{In}_{1-x} \mathrm{Ga}_{x}$ As dots. The Stransky-Krastanov growth mode is applied to a large variety of III-V and II-VI compounds. An interesting alternative for fabricating GaAs or InAs QDs is provided by a technique which is not strain driven, called molecular droplet epitaxy (Koguchi et al., 1991), see Fig.2(b) for a cross-sectional scanning tunneling microscopy image of a GaAs dot in AlGaAs (Keizer et al. 2010). The recently achieved high optical quality of GaAs droplet dots has allowed first investigations of carrier and nuclear spin dynamics (Belhadj et al., 2008, Sallen et al. 2011). Due to carrier confinement potentials between tens and hundreds of meV, the samples elaborated with the above techniques are suitable for optical spin manipulation often carried out at a temperature of $4 \mathrm{~K}$, with the possibility for detailed spectroscopy up to few tens of Kelvin.

This review will concentrate on the optical manipulation of spin states. A very high degree of control over carrier spin states and the mesoscopic nuclear spin system is also achieved in QDs defined by electrostatic potentials as summarized in the detailed review by Hanson et al. (2007). The electron (not hole) spin physics probed in these transport measurements at very low temperature $(100 \mathrm{mK})$ provide a powerful, complementary approach (Bluhm et al. 2010, Petta et al., 2005, Takahashi et al. 2011) to optical spectroscopy.

\section{B. Addressing individual charge states}

Controlling the charge state of QDs relies on the remarkable possibility of doping semiconductor materials with $n$-type or $p$-type impurities. In some cases the nonintentional residual doping is sufficient to obtain singlycharged QDs (Akimov et al. 2002, Belhadj et al. 2009), see Fig. 3(c), but usually a delta-doped layer is grown a few nanometers below the QD layer with a density ad- justed to reach the desired average QD charge Cortez et al., 2002, Greilich et al., 2006b; Laurent et al., 2006). This modulation doping technique can be significantly improved by controlling the chemical potential of the QD electrons with an electric voltage applied between the doped layer and a semitransparent top contact (Drexler et al. 1994). In these charge-tuneable structures a given QD is coupled to a reservoir of free carriers (a heavily doped layer) through a tunnel barrier as in Fig. 3(a). The energy levels of the QD can be adjusted with respect to the Fermi level in the highly doped barrier, to vary deterministically the charge state with the precision of a single elementary charge due to Coulomb blockade. This effect is clearly observed in micro-photoluminescence (PL) spectra by abrupt jumps of the (charged) exciton emission energy when the gate voltage is varied (see Fig. 3(b)) as a result of changes of the strong few particle direct Coulomb terms (Warburton et al., 2000)

\section{Electronic states, Optical selection rules and Carrier Coulomb exchange interactions}

The electronic structure of QDs can be analysed by techniques such as capacitance-voltage measurements, scanning-tunneling mircoscopy (Girard et al., 2009), electron-spin resonance, photo-current spectroscopy and a large variety of optical spectroscopy experiments. The latter allow a detailed study of the optically active electronic states and their symmetry by analysing the energy and polarization of absorbed or emitted photons. These experiments probe the interplay between carrier confinement, direct and exchange Coulomb terms and the hyperfine interaction. The orders of magnitude of the different effects that determine the optical and spin properties are given in tableI for the model system of InAs dots in GaAs.

QDs can be populated by valence holes and conduction electrons through optical excitation and/or through controlled tunneling in charge tuneable structures (Warburton et al., 1998). For a simplified calculation of optical transition energies between conduction band electron states and valence band hole states the single particle energies are determined by treating the electron-hole confinement potential within the harmonic approximation. For self-assembled as well as interface fluctuation dots the vertical confinement energies (along the growth axis $z$ ) are almost an order of magnitude larger than the lateral confinement energies in the $x y$ plane. The quantization energies of both electrons and holes are larger than the Coulomb energies. The Coulomb effects can therefore be treated as perturbations to the single particle structure (Warburton et al. 2000). At zero magnetic field the lowest lying conduction (valence) level $S_{c}\left(S_{v}\right)$ is twofold degenerate and the adjacent $P_{c}\left(P_{v}\right)$ level is fourfold degenerate in the case of axial symmetry, as in an ideal two-dimensional harmonic potential (Warburton 

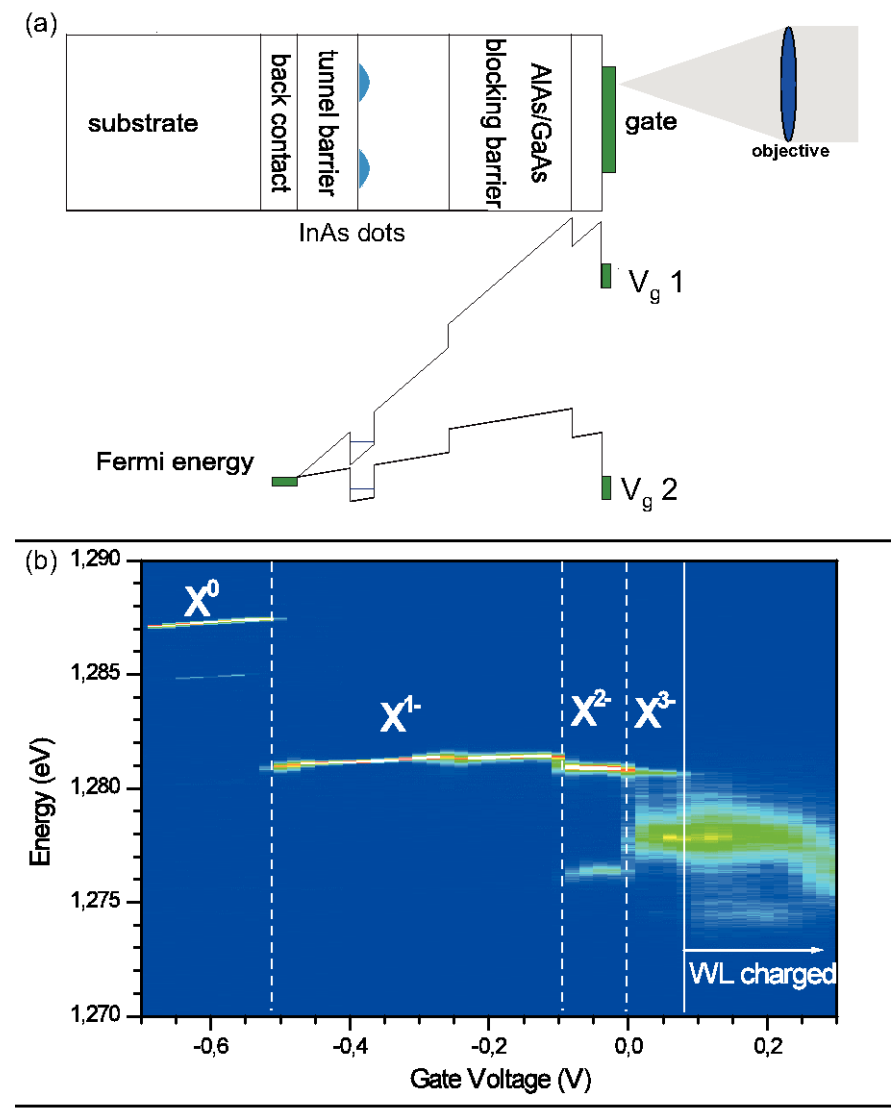

(c)

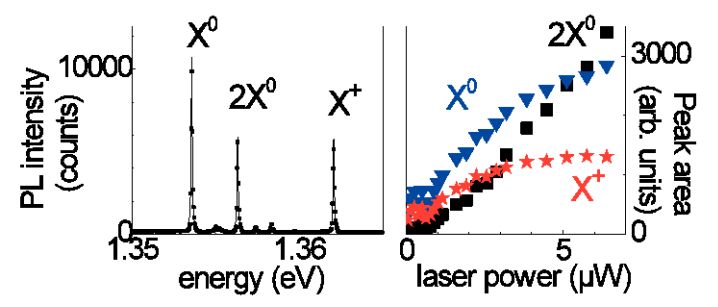

FIG. 3 Sample A (a) Scheme of InAs QDs embedded into a charge tunable device as in Warburton et al. (2000), where for a voltage $\mathrm{V}_{g 1}$ applied to the top gate the electronic level of the dot is above the Fermi energy of the highly n-doped back contact. The QD contains no conduction electron. For a gate voltage $\mathrm{V}_{g 2}$ the electronic level of the dot is now below the Fermi sea and an electron can tunnel into the dot. (b) The charging of a single InAs QD with electrons is accompanied by discrete jumps in the emission energy when going from the neutral exciton $\mathrm{X}^{0}$ (1 electron, 1 hole) to the charged exciton $\mathrm{X}^{-}$(2 electrons, 1 hole) etc until the wetting layer (WL) is charged. Sample B (c) Left: charge fluctuations (a doping hole or electron tunnel into and out of the dot) in non-intentionally doped dots allow the observation of neutral excitons $X^{0}$, charged excitons $X^{+}$and biexcitons $2 X^{0}$ in photoluminescence (PL) spectra that are integrated over seconds i.e. over times much longer than the charge fluctuation times (Belhadj et al. 2009) Right: In addition to the fine structure, the emission intensity of each transition as a function of optical excitation power allows to distinguish between different exciton complexes containing two, three or four optically generated charge carriers.
TABLE I Typical transition and interaction energies for a standard InAs QD in GaAs grown along the [001] axis, measured at a temperature of $4 \mathrm{~K}$, see for example Bayer et al. (2002); Urbaszek et al. (2003); Warburton et al. (1998), are listed. It is important to note that all properties which are linked to the QD size and shape and hence the exact confinement potential can vary considerably from dot to dot, this table merely indicates typical values for confinement energies, Coulomb interactions and Zeeman energies to establish the relative strength of the different interactions.

\begin{tabular}{ll}
\hline \hline interaction & energy in $\mathrm{eV}$ \\
\hline GaAs barrier & 1.519 \\
Electron to heavy hole transition in & 1.44
\end{tabular}
wetting layer

InAs dot electron to heavy hole 1.3 transition

electron confinement energy

heavy hole confinement energy

$50 \cdot 10^{-3}$

direct Coulomb interaction between two S electrons

exchange Coulomb interaction between an $\mathrm{S}$ and a $\mathrm{P}$ electron

fine structure splitting between $\mathrm{J}=2$ and $\mathrm{J}=1 X^{0}$ due to isotropic e-h Coulomb exchange interaction $\delta_{0}$

fine structure splitting of $\mathrm{J}=1 X^{0}$ due to anisotropic e-h Coulomb exchange interaction $\left|\delta_{1}\right|$

electron Zeeman splitting $\hbar \omega_{Z}^{e}$ at $B_{z}=1 \mathrm{~T}$

nuclear Zeeman splitting $\hbar \omega_{Z}^{n}$ at $B_{z}=1 \mathrm{~T}$

$$
\begin{aligned}
& 25 \cdot 10^{-3} \\
& 20 \cdot 10^{-3} \\
& 5 \cdot 10^{-3}
\end{aligned}
$$

$100 \ldots 500 \cdot 10^{-6}$

$0 \ldots 150 \cdot 10^{-6}$

$30 \cdot 10^{-6}$

$30 \cdot 10^{-9}$

et al. 1998), see Fig.2 for the energy level diagram. Here $S$ and $P$ refer to the symmetry of the envelope part of the Bloch function of the carrier state. For brevity, a Coulomb correlated electron-hole pair trapped inside a dot by the confinement potential will be called exciton in the following.

The electric-dipole interaction of an electromagnetic wave with carriers in a semiconductor is governed by strict optical selection rules (Meier and Zakharchenya, 1984). Energy and angular momentum are conserved for transitions between the valence and conduction band of a typical zincblende semiconductor like GaAs. The periodic part of the Bloch function of the conduction states is s-like, so the electron angular momentum is simply $m_{s}= \pm 1 / 2$ in units of $\hbar(\uparrow$ or $\downarrow$ ). The p-like valence states are determined by spin-orbit coupling and we consider here only the states with total angular momentum of $J=3 / 2$ as the split-off states $J=1 / 2$ are very far in energy (hundreds of meV in GaAs based samples) and can usually be neglected.

The quantization axis $z$ is chosen perpendicular to the QD plane and in most experiments $z$ is also parallel to the excitation light propagation direction. Following ab- 
sorption of a photon of suitable energy, an electron is promoted from a valence state to a conduction state. The absorption of a photon can increase the electron angular momentum by 1 for a $\sigma^{+}$polarized photon or lower it by 1 for a $\sigma^{-}$polarized photon, see Fig. 4 for all possible transitions between valence and conduction states in a simple picture. The selection rules for photon absorption and emission are identical. The unoccupied valence state left behind due to the promotion of the electron to the conduction state is called hole. The states with a projection of $J_{z}= \pm 3 / 2$ (介 or $\left.\Downarrow\right)$ are called heavy holes, $J_{z}= \pm 1 / 2$ are called light holes.

The heavy and light hole valence states are separated by an energy $\Delta_{H L}$ of typically several tens of meV due to quantum confinement and/or strain. For most of the experiments the light hole states can safely be ignored and optical exciton spin state preparation is straightforward. In practice however, strain, interface rotational symmetry breaking (Bester and Zunger, 2005; Grundmann et al. 1995, Krebs and Voisin, 1996) and shape anisotropy introduce heavy to light hole coupling which make all the transitions between the states indicated in Fig. 4 possible, yet with very different probabilities (Bayer et al., 2002, Belhadj et al., 2010, Koudinov et al. 2004 Léger et al., 2007).

Optical excitation of an empty dot with a suitable energy results in a transition from a valence to a conduction state and in the formation of a neutral exciton $X^{0}$, which allows to study carrier spin dynamics during the radiative lifetime of typically hundreds of picoseconds (Paillard et al. , 2000). For studies on longer time scales the spin information can be transferred to resident carriers in doped dots. In this review we focus on the three most relevant configurations: a conduction electron-valence hole pair $X^{0}$ with two optically active bright states ( $\downarrow$ or $\left.\Downarrow \uparrow\right)$ and two dark states ( $\uparrow$ or $\Downarrow \downarrow)$, the negatively charged exciton (trion) $\mathrm{X}^{-}(\Uparrow \uparrow \downarrow$ or $\Downarrow \uparrow \downarrow)$ and the positively charged exciton $\mathrm{X}^{+}(\Uparrow \Downarrow \uparrow$ or $\uparrow \downarrow \downarrow)$. Here $\uparrow(\downarrow)$ and $\uparrow(\Downarrow)$ represent the conduction electron spin and hole pseudo-spins, and for example $\Downarrow \uparrow \downarrow$ stands for $\frac{1}{\sqrt{2}}(\uparrow \downarrow-\downarrow \uparrow) \otimes \Downarrow$, where the antisymmetrization of the conduction states is more explicit. Due to strong localization of the carrier wave function, direct and exchange Coulomb, as well as correlation effects are very strong in dots. For the trions the direct and exchange Coulomb interaction lead to a renormalization of the transitions energies in the meV range but no fine structure splitting due to Kramers degeneracy (Bayer et al., 2002, Belhadj et al., 2008, Högele et al. 2004).

For neutral excitons (in zero magnetic field and in the absence of strong nuclear polarization) selection rules are affected by the electron hole Coulomb exchange interaction. This interaction includes an anisotropic contribution (Bayer et al. 2002, Tong and Wu, 2011) due to deviation of the real $\mathrm{QD}$ shape from a perfectly circular shape, see microscopy images in Fig.2 and/or due to the dot-semiconductor matrix interface anisotropy. Due to anisotropic exchange, $\mathrm{X}^{0}$ recombination results in a conduction electron states

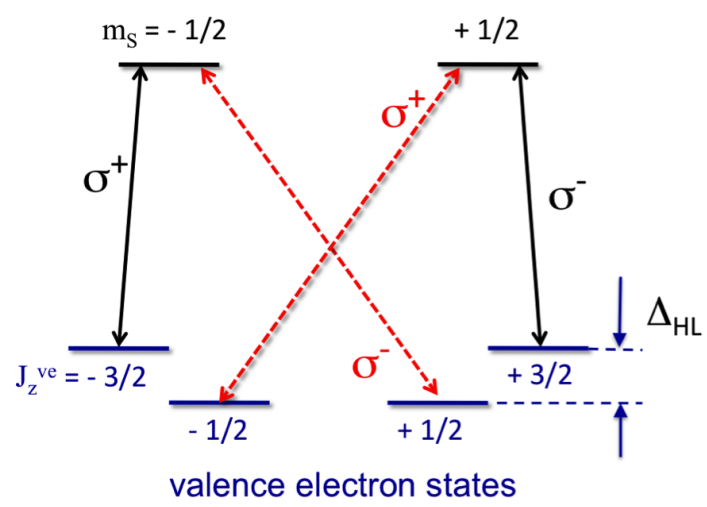

FIG. 4 Optical selection rules for inter-band transitions involving valence band electrons with angular momentum $J^{v e}=$ $3 / 2$ in units of $\hbar$, the corresponding photon polarization $\sigma^{+}$ or $\sigma^{-}$is indicated. Valence states with $J^{v e}=1 / 2$ are well separated in energy and are not shown here. Absorption of a $\sigma^{+}$polarized photon by a $J_{z}^{v e}=-3 / 2$ valence electron, results in the promotion of this electron to the conduction state $m_{s}=-1 / 2$ and a hole heavy hole $J_{z}=+3 / 2$ is left behind in the valence band. The energy separation between valence heavy and light hole states $\Delta_{H L}$ is typically several tens of $\mathrm{meV}$ in InAs dots in GaAs

doublet of linearly-polarized transitions, separated by an energy $\delta_{1}$ that varies from a few to a few tens of $\mu \mathrm{eV}$ from dot to dot in InAs/GaAs samples, see table I.

\section{Optical spectroscopy techniques}

To investigate the spin dynamics of carriers and nuclei a large variety of optical spectroscopy techniques have been developed, each adapted to the time scales relevant for the experiment. The typical radiative lifetime of a neutral or charged exciton is hundreds of picoseconds (Paillard et al., 2001, 2000), electron spin coherence times can be prolonged up to $200 \mu \mathrm{s}$ (Bluhm et al. 2010, Greilich et al. 2006b). Efficient collection of single dot photoluminescence following non-resonant excitation (Maletinsky et al. 2007a) and resonant fluorescence ( $\mathrm{Lu}$ et al. 2010) result in signal integration times well below the millisecond range, which provides the time resolution necessary to measure for example the nuclear polarization build-up time.

The discreteness of the QD energy states was demonstrated in optical spectroscopy experiments as early as 1994 (Marzin et al., 1994). Reducing the detection spot size in optical experiments to an area that contains only one nano-object permits studying directly the optical properties of an individual dot. A simple and powerful tool is non-resonant photoluminescence (PL), where carriers are optically excited in the surrounding semiconductor matrix by a laser tuned above the QD resonance energy i.e. either into wetting layer or barrier states. 
The carriers are subsequently trapped by the QD confinement potential and, following energy relaxation, recombine radiatively at the ground state energy, see $S_{c}$ to $S_{v}$ transition in Fig.2(c). More recently resonant fluorescence experiments where the excitation laser is resonant with the energy necessary for absorption from the highest lying valence level to the lowest lying conduction level (Muller et al. 2007) have shown beautiful analogies to atomic physics (Vamivakas et al., 2009). Two closely related, powerful techniques developed in charge tuneable structures are differential transmission and reflectivity, which also allow resonant probing of QD states (Alen et al., 2006; Högele et al., 2004). These experiments are carried out with pulsed or $\mathrm{cw}$ excitation. The challenge is to detect a very weak optical signal stemming from only one photon per recombination process. In practice efficient cw detection with Si-based CCD cameras and avalanche diodes are adapted to single dot measurements. For time resolved measurements and also to observe the spin physics in several thousand dots simultaneously experiments on QD ensembles are useful, that allowed important discoveries in the field, in resonant PL (Paillard et al. 2001), Kerr and Faraday rotation probing the real part of the refractive index (Greilich et al. 2006b) and photoinduced circular dichroism (Eble et al. $2009)$ probing the imaginary part of the refractive index. An interesting alternative to conventional pump-probe techniques is to passively detect the spectrum of intrinsic random spin fluctuations of carriers in thermal equilibrium (i.e., without optical pumping or initialization). This technique labelled spin noise spectroscopy has been successfully applied to electron (Crooker et al., 2010$)$ and hole spins (Dahbashi et al. 2012; Li et al., 2012), respectively, interacting with nuclear spins.

\section{E. Electron spin orientation mechanisms}

The target of this subsection is to explain how carrier spin states in QDs can be initialised in optical experiments. Two very different scenarios have to be distinguished: non-resonant and resonant optical excitation. The technical advantage of non-resonant optical excitation is the possibility to avoid blinding of the detector by the excitation laser light, thanks to spectral filtering. Also, contrary to atomic physics, non-resonant excitation is very efficient due to the high absorption probability of the thick barrier layer or $2 \mathrm{D}$ wetting layer. In the case of non-resonant excitation, the carriers have to relax in energy towards the quantum dot ground state $S_{v}$ for holes and $S_{c}$ for electrons. The average carrier spin $\left\langle S_{z}\right\rangle$ that can be initialised in the QD ground state is the result of the (i) spin initialisation upon photon absorption in the barrier or in the wetting layer according to the optical selection rules (ii) spin relaxation of the carrier during its presence in the barrier or wetting layer (iii) spin and energy relaxation during capture into the QD.
In spite of the expected phonon bottleneck ${ }^{1}$, it is observed that the carriers in the majority of samples relax on a picosecond time-scale towards the dot ground state (Verzelen et al., 2002). As a general rule, hole spin relaxation is efficient in bulk semiconductors and quantum wells (Damen et al., 1991; Dyakonov, 2008) i.e. during relaxation, whereas the electron keeps its spin orientation for longer and can to a high degree preserve its spin state during capture (Braun et al. 2005; Kalevich et al. 2001).

Excitation in the GaAs barrier for InAs dots (or the AlGaAs barrier for GaAs dots) involves both light and heavy hole transitions. As a result, a circularly polarized excitation creates both up and down electron spins (see Fig 4 for selection rules). The heavy-hole transition has a roughly 3 times larger oscillator strength than the light hole transition. As a result, under $\sigma^{-}$excitation for 3 spin $\uparrow$ electrons only 1 spin $\downarrow$ electron is created in a conduction state. This corresponds to an optical spin initialization of $\frac{n_{\uparrow}-n_{\downarrow}}{n_{\uparrow}+n_{\downarrow}}=\frac{3-1}{3+1}=50 \%$. To increase the optically generated average spin, excitation into the bi-dimensional wetting layer (if present in the sample) allows in principle injection of $100 \%$ spin polarized electrons when driving heavy hole transitions, which are separated in energy from the light hole transitions in the wetting layer due to confinement and/or strain.

The neutral exciton $X^{0}$.- For an empty QD, ground state electron and hole form an $X^{0}$. Due to the strong overlap of the carrier wavefunctions, Coulomb correlations are important and the anisotropic part of the exchange interaction results in two linearly polarized exciton eigenstates that are separated in energy by $\delta_{1}$ Gammon et al. (1996). Assume that the QD is excited with a pulsed laser (temporal pulse length $\tau_{L}$ ) that is $\sigma^{+}$polarized and for which $\hbar / \tau_{L}>\delta_{1}$ holds. Due to anisotropic exchange, the created exciton is not in an $X^{0}$ eigenstate, but in a superposition of the linearly polarized eigenstates, so during the radiative $X^{0}$ lifetime $\tau_{r}$ quantum beats in the $\sigma^{+/-}$basis are observed, for a detailed discussion see (Sénès et al., 2005). If the beat period $\approx \hbar / \delta_{1} \ll \tau_{r}$, then the time averaged circular polarization degree $\frac{I_{\sigma^{+}}-I_{\sigma^{+}}}{I_{\sigma^{+}+I_{\sigma^{+}}}}$, where $I_{\sigma^{+}}\left(I_{\sigma^{-}}\right)$are the $\sigma^{+}\left(\sigma^{-}\right)$ polarized emission intensities, will tend to zero. In general $\hbar / \delta_{1}$ and $\tau_{r}$ can be of similar magnitude, which leads in $\mathrm{cw}$ experiments to a decrease of exciton pseudo-spin polarization from initially $\rho_{c}^{0}$ down to $\rho_{c}$ during the radiative lifetime as $\rho_{c}=\rho_{c}^{0}\left(1+\omega^{2} \tau_{r}^{2}\right)^{-1}$ with $\hbar \omega=\delta_{1}$.

\footnotetext{
1 At first sight energy relaxation from the continuous barrier states to the quantum dot ground states via discrete states separated in energy by tens of meV seems very unlikely if the relevant energy level spacing does not exactly match the energy of lattice phonons. The anticipated slowing down of the relaxation via phonon emission is termed phonon bottleneck, but is rarely observed in practice as, for example, the involvement of polarons assures energy conservation during relaxation (Verzelen et al. 2002 .
} 
The positively charged exciton $X^{+}$.- In the case of the $X^{+}$exciton, a doping hole is present before the optically generated electron and hole are captured. The incoming hole spin is random, so the resident hole (which has a given spin orientation) and the optically generated hole can form a hole pseudo spin singlet. As a result, the subsequent evolution of the spin orientated electron can be monitored during the $X^{+}$lifetime (Krebs et al. 2008 ; Laurent et al. 2005) in the absence of Coulomb exchange effects. Recording the $X^{+}$emission from an InAs dot, initialisation of electron spin polarization as high as $80 \%$ $\left(\left\langle S_{z}\right\rangle=0.4\right)$ has been achieved through non-resonant excitation into the wetting layer, about $100 \mathrm{meV}$ above the dot ground state (Urbaszek et al. 2007 ).

The negatively charged exciton $X^{-}$.- Non-resonant excitation with a circularly polarized laser of a dot doped with a resident electron is in principle not expected to yield polarized emission, as the incoming electron will form a spin singlet with the resident electron (total spin $S=0$ ). The hole spin, completely randomised, will determine the polarization of the emitted photon after $X^{-}$recombination. But surprisingly, this prediction has not been confirmed in experiments; instead, non-resonant circularly-polarized excitation of a QD results in a partially polarized ground state emission with an helicity opposite to that of the excitation (Cortez et al. 2002 , Dzhioev et al., 1998b; Laurent et al., 2006, Oulton et al. 2007: Shabaev et al. 2009). The origin of this negative polarization has been ascribed to exchange related electron-hole spin flip-flop processes during carrier energy relaxation (Laurent et al., 2006, Ware et al., 2005). Another possible scenario involving the accumulation of dark excitons in the barriers, that are subsequently captured by the dots resulting in negative polarization is likely to be applicable to GaAs interface fluctuation dots (Bracker et al. 2005). Independent of its origin, changes in the negative polarization degree observed for $X^{-}$initialisation and subsequent recombination, can be used as a sensitive probe for nuclear spin effects Auer et al. 2009).

Strictly resonant and quasi-resonant optical excitation.- Although experimentally simple, non-resonant excitation has several disadvantages: (i) Initialisation of the QD in a well defined coherent superposition of polarization states is not possible, as coherence is lost during relaxation. (ii) As carriers with well defined spin orientation have been injected into the barrier and/or wetting layer material, the electron can interact with the nuclear spins during its presence in these layers. If the QD emission shows that nuclear spins in the dot are polarized, in the case of non-resonant excitation one cannot be $100 \%$ sure that this polarization originates from nuclear spins in the QD only, or, if the QD is simply a nanoscopic probe of a macroscopic nuclear spin polarization created inside the sample through spin diffusion (Paget, 1982). A first step to circumvent these problems is to use what is termed quasi-resonant excitation, for example 1LO phonon energy above the $\mathrm{S}_{v}-\mathrm{S}_{c}$ transition or

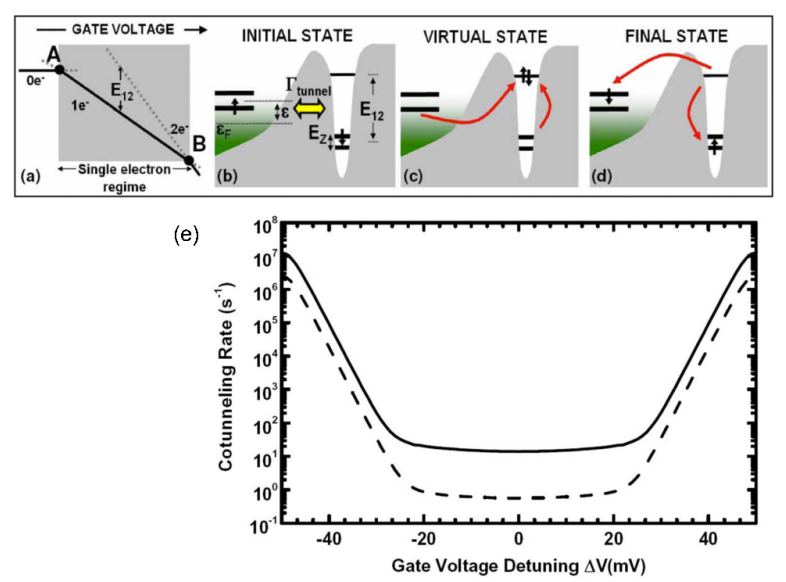

FIG. 5 (a) to (d) a QD exchanges an electron with the reservoir via a virtual two electron state and (e) calculation of the co-tunneling rate as a function of gate voltage after Dreiser et al. (2008) for tunneling rates $0.1 \mathrm{~ns}^{-1}$ (solid curve) and $0.02 \mathrm{~ns}^{-1}$ (dashed curve).

directly the $\mathrm{P}_{v}-\mathrm{P}_{c}$ transition. Reaching the ground state via emission of a single LO phonon partially preserves coherence (Flissikowski et al., 2001, Scheibner et al., 2003, Sénès et al., 2005$)$. In addition, as in these experiments the photon is absorbed directly by the QD states, one can be sure that the nuclear spins in the QD are the direct source of the detected nuclear spin polarization. This is demonstrated, for example, by illuminating the dot with a fixed laser polarization and changing the sign of the nuclear polarization by varying the gate voltage applied to a charge tunable structure i.e. by going from the $\mathrm{X}^{+}$to the $\mathrm{X}^{-}$emission (Eble et al., 2006, Lai et al. 2006). Resonant experiments allow precise control over the created spin state or superposition of states (Greilich et al. 2006a). Whereas in non-resonant experiments the hyperfine interaction will have negligible influence on the photon absorption probability, in resonant experiments under certain conditions the hyperfine interaction determines the polarization and energy of the preferentially absorbed photons (Chekhovich et al., 2010a, Gerardot et al. , 2008, Kloeffel et al. | 2011; Klotz et al., 2010; Latta et al. (2009), as discussed in Sec.V.C. Once carrier spin initialisation has been achieved, the spin will interact with its nuclear spin environment during its lifetime.

Electron co-tunneling in charge tunable structures.Our main focus in this review is on the interaction with nuclear spins, but before going into details an important spin interaction present in charge tunable structures has to be mentioned. An electron in a dot embedded in a charge tuneable structure is coupled to the continuum of delocalised electron states in the n-doped layer (Fermi sea) via the tunnel barrier. The physical problem itself of a single spin coupled coherently to the Fermi sea has parallels to the Kondo effect (Dreiser et al., 2008; Latta et al. 2011a; Smith et al., 2005). Essentially, each charge 
state corresponds to the ground state for a given gate voltage range $\Delta V=V_{\text {start }}-V_{\text {end }}$ over typically several tens of $\mathrm{mV}$. Close to $V_{\text {start }}$ and $V_{\text {end }}$ the exchange coupling to the electron reservoir is strong; in between it is weak (charging plateau), see Fig.5e. When the coupling is strong the electron spin can flip via an intermediate virtual transition either to an empty or doubly-occupied QD state, as shown in Fig.5(a)-(d) (Dreiser et al., 2008). The net result of exchanging an electron with the reservoir is a spin flip of the electron inside the dot characterized by a spin flip co-tunneling rate. So for experiments where stable carrier spins are required, the structures must be operated at a gate voltage close to the centre of the charging plateau where the co-tunneling rate is low and hence the spin state of the resident electron is long-lived.

\section{ELEMENTARY INTERACTIONS WITH NUCLEI IN QUANTUM DOTS}

Nuclear spin effects are important for experiments in QDs that investigate optical carrier spin manipulation. Three striking examples are shown in Fig.6. Figures (a) and (b) show that the Zeeman splitting of an exciton in a longitudinal magnetic field $B_{z}$ depends in a strongly non-linear fashion on the laser excitation power as nuclear spins start to get polarized (Tartakovskii et al. 2007), see Sec.V. These measurements show that hyperfine effects in III-V dots are of the same order as the fine structure of bright excitons, see also Fig.6e and tableI. Figure 6(c) shows absorption of the charged exciton $X^{-}$line at zero Tesla, with a text book Lorentzian lineshape. Figure 6(d) represents a highly unusual absorption spectrum at $B_{z}=4.5 \mathrm{~T}$, that is strongly broadened, asymmetric and changes with laser scan direction (Latta et al. , 2009). In this experiment nuclear spin polarization allows the QD transitions to be locked to the driving laser field as it changes frequency, see section V.C.3. So nuclear spin effects have to be taken into account even in a simple measurement of the transition energy of the $X^{-}$in an applied magnetic field. A direct proof that this type of behaviour is related to nuclear spins comes from original ODNMR measurements by Gammon et al. (1997), as detailed in section VI.E The electron Zeeman splitting in a longitudinal field of $B_{z}=1 \mathrm{~T}$ for single GaAs/AlGaAs dots changes, as nuclear spins are depolarized by a chirped radio-frequency source ( $\mathrm{RF}$ on) scanning the nuclear spin resonances for Ga and As, see Fig.6(e). The measurements show that the dynamic nuclear polarization created through optical pumping has an effect on the electrons that is comparable to the applied magnetic field, see Sec. $\mathrm{V}$.

Below we highlight the basics of the magnetic and electrostatic coupling between electrons and nuclei that will allow us in the following sections to interpret quantitatively, whenever possible, the fascinating nuclear spin effects observed in optical spectroscopy experiments in quantum dots for a wide range of experimental condi-
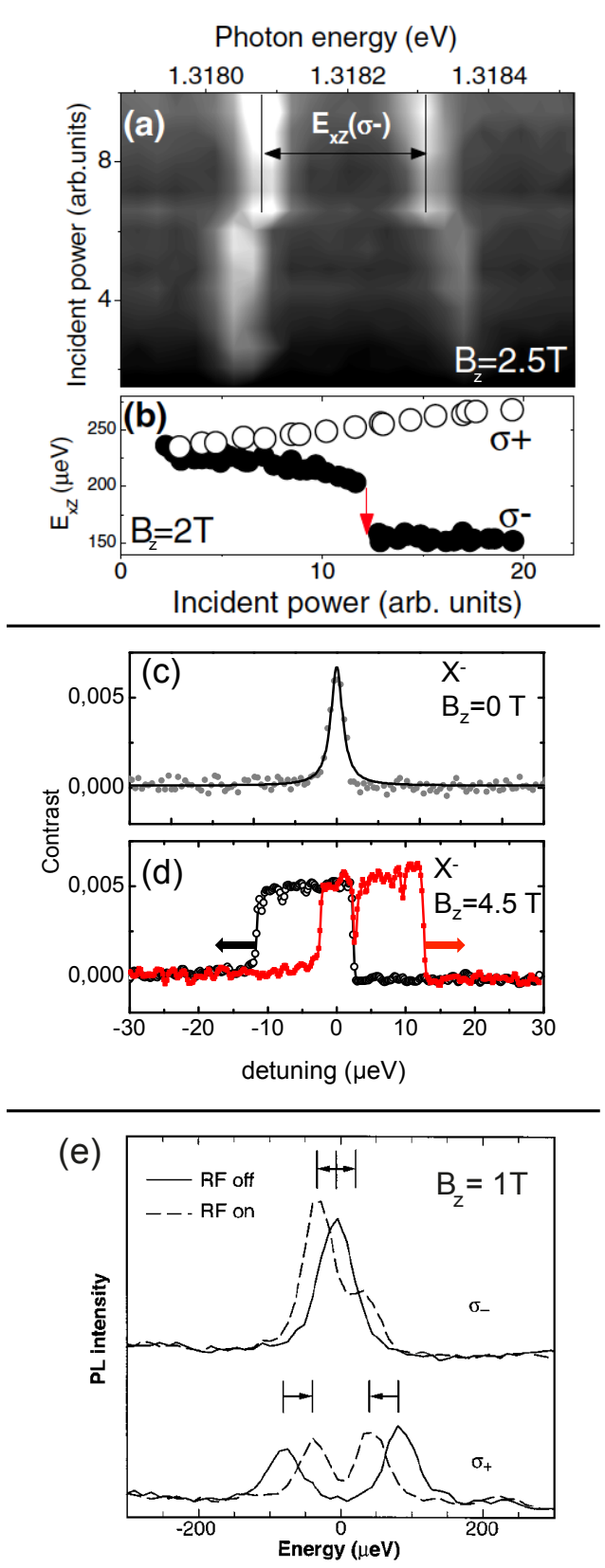

FIG. 6 (a) Gray-scale plot showing exciton PL spectra recorded for an individual InGaAs dot. The spectra are recorded at $B_{z}=2.5 \mathrm{~T}$ using unpolarized detection. (b) power dependences measured at $B_{z}=2 \mathrm{~T}$ for $\sigma^{+}$and $\sigma^{-}$ excitation polarizations (after Tartakovskii et al. (2007)) (c) trion $X^{-}$absorption at zero magnetic field with Lorentzian fit of linewidth $2 \mu \mathrm{eV}$ and strong deviation from Lorentzian lineshape at $B_{z}=4.5 \mathrm{~T}$ (d) after Latta et al. (2009). (e) Zeeman splitting for a single GaAs interface fluctuation QD. Due to dynamic nuclear polarization the Zeeman splitting for $\sigma^{+}$and $\sigma^{-}$polarized excitation are different. Randomizing the nuclear spin orientation with a radiofrequency field (RF on) results in the same Zeeman spliting for both laser polarizations, after Gammon et al. (1997). 
TABLE II Hyperfine constants in GaAs, InAs, InP and CdTe for a cell containing two atoms, see Testelin et al. (2009) and references therein. Please note that an average is quoted for $\mathrm{Ga}$ and In for which two stable isotopes exist.

\begin{tabular}{llll}
\hline \hline isotope & $\begin{array}{l}\text { nuclear } \\
\text { spin I }\end{array}$ & $\begin{array}{l}\text { abundance } \\
(\%)\end{array}$ & $\begin{array}{l}\text { hyperfine } \\
\text { constant } \\
\text { A in } \mu \mathrm{eV}\end{array}$ \\
\hline $\mathrm{In}$ & $9 / 2$ & 100 & 56 \\
$\mathrm{Ga}$ & $3 / 2$ & 100 & 42 \\
$\mathrm{As}$ & $3 / 2$ & 100 & 46 \\
$\mathrm{P}$ & $1 / 2$ & 100 & 44 \\
$\mathrm{Cd}$ & $1 / 2$ & 25 & -30 \\
$\mathrm{Te}$ & $1 / 2$ & 8 & -45 \\
& & & \\
\hline \hline
\end{tabular}

tions.

\section{A. Magnetic coupling of electrons to nuclei: Hyperfine interaction}

The strength of the hyperfine interaction in QDs is enhanced compared to semiconductor bulk or quantum well structures due to the strong localization of the electron wavefunction over typically only $10^{5}$ lattice sites. This number is too small for efficient cancellation of the total nuclear spin by averaging (Burkard et al., 1999: Merkulov et al., 2002), yet too large to address each nuclear spin state individually. In III-V QDs like GaAs, InP and InAs, $100 \%$ of the lattice sites have a non-zero nuclear spin and these materials are taken here as model systems. Even for solids with very few isotopes carrying a nuclear spin like diamond (Childress et al. 2006), ZnO (Liu et al. 2007) or CdSe (Feng et al., 2007) hyperfine effects still play a key role in the carrier spin state evolution.

There are two main contributions to the hyperfine interaction (Abragam, 1961): (i) The Fermi contact interaction is efficient when there is a physical overlap of the carrier wavefunction with the lattice site. This type of interaction is dominant for $s$-type wave functions (periodic part of the Bloch function) of conduction electrons. (ii) The dipole-dipole interaction is effective for p-type (non-zero orbital angular momentum) wave functions. This term is therefore dominant for valence-band states (holes). It is about one order of magnitude weaker than the Fermi contact interaction for conduction electrons (Chekhovich et al., 2011a; Desfonds et al., 2010, Eble et al. |2009: Fallahi et al., 2010, Fischer et al. 2008). In the bulk of this paper, namely sections [IV], [V] VI we concentrate on the interaction of a conduction electron with nuclear spins, the interaction between valence holes and nuclear spins will be discussed separately in Sec.VII.

To introduce the orders of magnitude of the energy shifts due to the hyperfine interaction between electron and nuclear spins, a comparison with the Zeeman splitting of the spin levels in an external magnetic field $\boldsymbol{B}=$
TABLE III Electron Overhauser splitting in $\mu \mathrm{eV}$ for 100 $\%$ nuclear polarization $\hbar \omega_{O S}^{e}=I^{\mathrm{Ga}} A^{\mathrm{Ga}}+I^{\mathrm{As}} A^{\mathrm{As}}$ for $\mathrm{GaAs}$ and InAs quantum dots, using the nuclear spin and hyperfine constant values from table II

\begin{tabular}{ll}
\hline \hline InAs & 315 \\
$\mathrm{In}_{0.5} \mathrm{Ga}_{0.5} \mathrm{As}$ & 230 \\
$\mathrm{GaAs}$ & 135 \\
\hline \hline
\end{tabular}

$\left(0,0, B_{z}\right)$ is helpful (Abragam, 1961; Dyakonov, 2008): The Zeeman energy of an electron spin with $\hat{S}_{z}^{e}=\frac{1}{2} \hat{\sigma}^{e}$ is

$$
\hat{H}_{Z e}=\mu_{B} g_{e} B_{z} \hat{S}_{z}^{e}=\hbar \omega_{Z}^{e} \hat{S}_{z}^{e}
$$

where $g_{e}$ is the longitudinal electron g-factor and $\mu_{B}=$ $9.27 \times 10^{-24} \mathrm{~J} / \mathrm{T}=58 \mu \mathrm{eV} / \mathrm{T}$. The Zeeman energy of a system of nuclear spins $I^{j}$ is given by:

$$
\hat{H}_{Z N}=-\mu_{N} \sum_{j} g_{N j} B_{z} \hat{I}_{z}^{j}
$$

summing over all nuclei $j$ in the system. Here $g_{N}$ is the nuclear g-factor and $\mu_{N} \simeq \frac{\mu_{B}}{2000}$ is the nuclear magneton. For an order of magnitude calculation, we take the example of Indium and an electron g-factor of 0.6, and find $\left(g_{e} \mu_{B}\right) /\left(g_{N} \mu_{N}\right) \simeq 1000$. The energy separation between the nuclear spin states is therefore negligible compared to that of the electron spins.

The Fermi contact $(f c)$ hyperfine interaction in a QD between an electron spin and the $N$ nuclei of the atoms forming the dot is (Abragam, 1961, Gammon et al. 2001):

$$
\hat{H}_{h f}^{f c}=\frac{\nu_{0}}{2} \sum_{j} A^{j}\left|\psi\left(\boldsymbol{r}_{j}\right)\right|^{2}\left(2 \hat{I}_{z}^{j} \hat{S}_{z}^{e}+\left[\hat{I}_{+}^{j} \hat{S}_{-}^{e}+\hat{I}_{-}^{j} \hat{S}_{+}^{e}\right]\right)
$$

where $\nu_{0}$ is the two atom cell volume, $\boldsymbol{r}_{j}$ is the position of the nuclei $j$ with spin $\hat{I}^{j}$ and $\psi\left(\boldsymbol{r}_{j}\right)$ is the normalized electron envelope function. The nuclear spin is $3 / 2$ for $\mathrm{Ga}$ and $\mathrm{As}, 5 / 2$ for $\mathrm{Al}, 9 / 2$ for In in units of $\hbar . A^{j}$ is the constant of the hyperfine interaction with the electron in the order or $50 \mu \mathrm{eV}$ for In, Ga and As, see table II.

As an electron interacts simultaneously with about $10^{5}$ lattice sites, one can consider in the mean field approach that the electron spin is affected by a mean nuclear spin polarization $\left\langle\hat{\boldsymbol{I}}^{j}\right\rangle$ acting like an effective magnetic field $B_{n}$ (Overhauser field) :

$$
\boldsymbol{B}_{n}=\frac{\nu_{0} \sum_{j} A^{j}\left|\psi\left(\boldsymbol{r}_{j}\right)\right|^{2}\left\langle\hat{\boldsymbol{I}}^{j}\right\rangle}{g_{e} \mu_{B}}
$$

For uniform nuclear polarization, the field $B_{n}$ is independent of the electron localization volume and is in the order of $B_{n}^{\max } \simeq 5 \mathrm{~T}$ for fully polarized nuclei in GaAs (Paget et al. 1977), as the maximum Overhauser shift is simply $g_{e} \mu_{B} B_{n}^{\max }=I^{G a} A^{G a}+I^{A s} A^{A s}=135 \mu \mathrm{eV}$.

The hyperfine interaction is reciprocal, see scheme in Fig.1. so also the nuclei are effected by the average electron spin polarization acting like an effective magnetic 
field $B_{K}$ (Knight field). The time averaged Knight field acting on one specific nucleus $j$ is given by:

$$
\boldsymbol{B}_{K j}=f_{e} \frac{\nu_{0} A^{j}}{g_{N} \mu_{N}}\left|\psi\left(\boldsymbol{r}_{j}\right)\right|^{2}\left\langle\hat{\boldsymbol{S}}^{e}\right\rangle
$$

where $f_{e}$ is the filling factor $\in[0,1]$ characterizing the occupation of the dot by electrons, underlining that the Knight field is zero in the absence of electrons. The maximum Knight field can be estimated as $B_{K}^{\max } \simeq$ $\frac{B_{n}^{\max }}{N} \frac{g_{e} \mu_{B}}{g_{N} \mu_{N}}$, so for $N \simeq 10^{5}$ results in $B_{K}^{\max }$ in the tens of $\mathrm{mT}$ range. The amplitude of the Knight field for a nucleus situated in the centre of the dot (where electron occupation probability is strongest) will be higher than for a nucleus in the dot periphery. The Knight field experienced by the nuclei leads to frequency shifts in ODNMR spectra of individual QDs (Brown et al. 1998).

Introducing $\tilde{A}$ as the average of the hyperfine constants $A^{j}$ and assuming a strongly simplified, uniform electron wavefunction $\psi(\boldsymbol{r})=\sqrt{2 /\left(N \nu_{0}\right)}$ over the involved nuclei, Eq. 3 simplifies to:

$$
\hat{H}_{h f}^{f c}=\frac{2 \tilde{A}}{N}\left(\hat{I}_{z} \hat{S}_{z}^{e}+\frac{\hat{I}_{+} \hat{S}_{-}^{e}+\hat{I}_{-} \hat{S}_{+}^{e}}{2}\right)
$$

where $\hat{I}=\sum_{j=1}^{N} \hat{I}^{j}$.

The energy level splittings between the different nuclear and electron spin states are determined by the hyperfine interaction in combination with the applied magnetic field $B_{z}$. $\hbar \omega_{O S}=2 \tilde{A}\left\langle\hat{I}_{z}\right\rangle / N=\mu_{B} g_{e} B_{n}$ relates the Overhauser shift $\hbar \omega_{O S}$ to the average nuclear polarisation. We can therefore access the average nuclear polarisation by measuring $\hbar \omega_{O S}$ in single dot spectroscopy, as in Fig.6e. For example, when the nuclear spins are polarized (i.e. the RF source is off) the total electron Zeeman splitting $\hbar \omega_{e}$ in Fig.6e is given by $\hbar \omega_{e}=\hbar\left(\omega_{Z}^{e}+\omega_{O S}^{e}\right)$. When the RF source is on, the nuclei are depolarized, the Overhauser field $B_{n}$ is vanishingly small and $\hbar \omega_{e}=\hbar \omega_{Z}^{e}$. The difference between the two cases allows to measure the Overhauser shift $\hbar \omega_{O S}^{e}$.

The hyperfine interaction is time dependent since the electron lifetime is finite and its spin may also relax during its lifetime. The time dependence of the second term in Eq. 6 can be explicitly written as: $\hat{H}_{1}(t)=$ $\frac{\tilde{A}}{N}\left(\hat{I}_{+} \hat{S}_{-}^{e}+\hat{I}_{-} \hat{S}_{+}^{e}\right) h_{1}(t)$. This term allows for spin transfer via simultaneous spin flips (flip-flop) of a carrier and nuclear spin. As the nuclear Zeeman splitting is negligible, the electron Zeeman splitting plays a crucial role in determining the probability of these spin flip-flops, as already pointed out in the original paper by Overhauser (1953). It should be emphasized that while the term $\propto \hat{I}_{z} \hat{S}_{z}^{e} h_{1}(t)$ also fluctuates in time, it does not directly induce any spin flips. Depending on the exact experimental conditions, the electron-nuclear spin flip-flop term can lead to electron spin dephasing (Braun et al. 2005), dynamic nuclear polarization (Gammon et al., 1997) or nuclear spin dephasing (Abragam, 1961, Merkulov et al., 2002).
$\hat{H}_{1}(t)$ can be visualized as a random perturbation between states split in energy by $\hbar \omega_{e}$. The function $h_{1}(t)$ is characterized by its mean value $\overline{h_{1}(t)}=f_{e}$ and a simple, auto-correlation function $\overline{h_{1}(t) h_{1}^{*}(t+\tau)}=\exp \left(-\frac{|\tau|}{\tau_{c}^{e}}\right)$ with a correlation time $\tau_{c}^{e}$. The fraction of time the quantum dot contains an electron $f_{e}$ takes values between 0 and 1 . The rate of nuclear polarisation will depend on the splitting $\hbar \omega_{e}$ and the level broadening $\hbar / \tau_{c}^{e}$, (Eble et al. 2006, Urbaszek et al. 2007), as discussed in Sec.V.

For commonly achieved nuclear spin polarization values well below 100\%, the nuclear field fluctuates around a mean value $\left\langle B_{n}\right\rangle$. The fluctuations (root mean square deviation) can be written as an effective field $\delta B_{n}=$ $\sqrt{\left\langle B_{n}^{2}\right\rangle-\left\langle B_{n}\right\rangle^{2}}$. Several theoretical studies have predicted that the dominant mechanism of electron spin relaxation in QDs at low temperature and zero external magnetic field is due to the hyperfine interaction with these nuclear field fluctuations $\delta B_{n}$ (Burkard et al., 1999 ; Khaetskii et al., 2002; Merkulov et al., 2002; Semenov and Kim, 2003). The reason for the non-negligible $\delta B_{n}$ lies in the finite number of nuclei within the dot: The mesoscopic nuclear spin system of a QD is described by the nuclear spin operators $\hat{I}_{x}, \hat{I}_{y}, \hat{I}_{z}$. These operators do not commute, it is therefore impossible to determine the $x, y$ and $z$ components of the nuclear spin system with equal precision i.e. they can not all be exactly zero. In the absence of DNP repeated measurements of the expectation value of $B_{n}$ at time intervals longer than the nuclear spin correlation time of the order of $10^{-4} \mathrm{~s}$ give an average of $\left\langle B_{n}\right\rangle=0$. But, employing a useful qualitative physical picture ${ }^{2}$, an electron spin will interact during its lifetime (about $1 \mathrm{~ns}$ in InAs QDs) with a field of typical magnitude $\delta B_{n}$ and random orientation during about $10^{-4} \mathrm{~s}$; this is referred to as the frozen fluctuation model (Merkulov et al., 2002) and is detailed in Sec.IV.

An important interaction between nuclear spins is the dipole-dipole interaction that allows for example nuclear spin diffusion in bulk GaAs samples (Paget, 1982) with spatially inhomogeneous nuclear polarization. The dipole-dipole interaction of a nucleus $n$ with the other nuclei $n^{\prime}$ separated by the translation vector $\boldsymbol{r}_{n n^{\prime}}$ can be written as (Abragam, 1961):

$$
\hat{H}_{d d}=\frac{\mu_{N}^{2}}{2} \sum_{n \neq n^{\prime}} \frac{g_{n} g_{n^{\prime}}}{r_{n n^{\prime}}^{3}}\left(\hat{\boldsymbol{I}}^{n} \hat{\boldsymbol{I}}^{n^{\prime}}-3 \frac{\left(\hat{\boldsymbol{I}}^{n} \boldsymbol{r}_{n n^{\prime}}\right)\left(\hat{\boldsymbol{I}}^{n^{\prime}} \boldsymbol{r}_{n n^{\prime}}\right)}{r_{n n^{\prime}}^{2}}\right)
$$

As a result of the dipole-dipole interaction each nucleus experiences a fluctuating local effective magnetic field $\delta B_{L}$, where $\delta B_{L} \simeq 0.15 \mathrm{mT}$ in GaAs, created by the other nuclei. Via the non-secular (non spin conserving) part of the dipole-dipole interaction nuclear spin is

\footnotetext{
2 The electron really interacts with a quantum field of indeterminate magnitude and direction at any time scale for $B=0$.
} 
transferred to the crystal as a whole and is not conserved, see Ch. VIII.E of Abragam (1961) were secular and nonsecular parts of the dipole-dipole interaction are detailed. The precession of the nuclear spins around $B_{L}$ is one of the reasons why dynamic nuclear polarization in GaAs bulk in the absence of any applied magnetic field is not possible (Meier and Zakharchenya, 1984). In QDs two interactions, namely the Knight field $B_{K}$ and the nuclear quadrupole interaction can in principle dominate $B_{L}$ already at zero field, as discussed in detail in Sec.III.B and V.B.5

\section{B. Electrostatic coupling: Nuclear quadrupole effects}

Due to lattice strain and atomic inter-diffusion electric quadrupolar effects are strong for nuclei in QDs compared to the influence of alloy disorder in unstrained bulk samples and hence play a central role in nuclear spin dynamics in QDs (Dzhioev and Korenev, 2007), see Bulutay (2012) for a detailed discussion. Quadrupolar effects are at the heart of many of the surprising effects that go beyond the nuclear spin physics known from bulk and quantum well systems, such as for example zero field DNP (Lai et al., 2006: Oulton et al., 2007) (see Sec, V.B.5), strongly suppressed spin diffusion (Maletinsky et al. , 2009) (see Sec VI.F), the anomalous Hanle effect (Krebs et al., 2010) (see Sec/VI.D) and the locking of quantum-dot resonances to an incident laser (Högele et al. 2012, Latta et al. 2009) (see Sec V.C.3).

Nuclei have no electric dipole moment and are thus insensitive to homogeneous electric fields (Abragam, 1961). But the non-spherical (prolate) charge distribution of atomic nuclei with spin $I>1 / 2$ presents an electric quadrupolar moment, as sketched in Fig.7k, which can couple to inhomogeneous electric fields produced by electron clouds, expressed as an electric field gradient $\partial^{2} V / \partial x_{\alpha} \partial x_{\beta}$ where $V$ is the electrical potential due to local charge distribution. If the nuclear environment has cubic symmetry, the electric field gradient vanishes and so does the quadrupolar coupling (Abragam, 1961: Slichter, 1990). This situation prevails in bulk GaAs, but the cubic symmetry breaks down in self-assembled QDs like InAs/GaAs because of large biaxial strain associated with the $\sim 7 \%$ lattice mismatch between InAs and GaAs. Also, inter-diffusion of In and Ga atoms during QD growth results in a substantial fraction of As atoms for which all first neighbours are no longer identical. The local tetrahedral symmetry is then lost and an electric field gradient arises along one of the crystallographic directions $\langle 111\rangle$ or $\langle 100\rangle$ (Meier and Zakharchenya, 1984).

This electrostatic coupling of the electronic system with the nuclear spin system can manifest itself in principle in the analysis of either system. For the conduction electrons most relevant for the physics described here the quadrupolar interaction vanishes (for $s$-electrons i.e. $l=0$ ) (Abragam, 1961) and in what follows we only consider the effect of the electric field gradients on the (a)
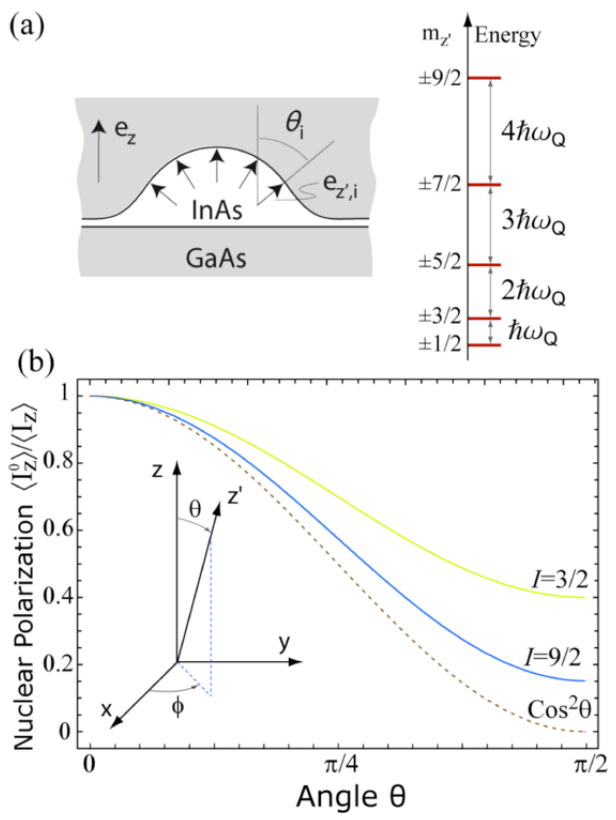

(c)

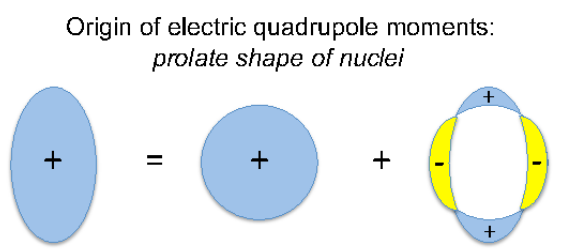

FIG. 7 (a) Sketch of the strain distribution in an InAs/GaAs QD and splitting of energy levels in zero field due to axially symmetric quadrupolar interaction for a nuclear spin $I=9 / 2$. (b) Reduction factor of nuclear polarization along $z$ due to the inclination $\theta$ of the quadrupolar main axis. In the approximation of high spin temperature, it is given by $\left\langle I_{z}\right\rangle /\left\langle I_{z}^{0}\right\rangle=$ $(I+3 / 4)(I-1 / 2) \cos ^{2} \theta /(I(I+1))+3(I+1 / 2) /(4 I(I+1))$ (c) A nucleus with non-spherical, prolate charge distribution is equivalent to a spherically symmetric charge distribution plus some positive charge shared between the two polar regions and a band of equal negative charge added around the equator. This addition has no dipole moment but does have electric quadrupole moment, after Williams (1991).

nuclear spin system. For simplicity we assume that the electric field gradients in the dot have cylindrical (axial) symmetry i.e. the strength of the interaction does not depend on the angle $\phi$ in the $x-y$ plane, defined in Fig. $7 \mathrm{~b}$ with respect to the growth axis $z$. The influence of the electric field gradients oriented along an axis $z^{\prime}$ on a nucleus of spin $I$ can then be described by Abragam, 1961):

$$
\hat{H}_{\mathrm{Q}}=\frac{\hbar \omega_{\mathrm{Q}}}{2}\left(\hat{I}_{z^{\prime}}^{2}-\frac{I(I+1)}{3}\right)
$$

where $\hbar \omega_{\mathrm{Q}}$ is the quadrupolar splitting proportional to the nuclear quadrupolar moment and to the electric field 
gradient, and $\hat{I}_{z^{\prime}}$ is the angular momentum projection on the principal axis $z^{\prime}$ of the electric field gradient. Without loss of generality one can assume $\hat{I}_{z^{\prime}}=\hat{I}_{z} \cos \theta+\hat{I}_{x} \sin \theta$ to analyze the effect of the Eq. (8) on the nuclear polarization:

$$
\begin{aligned}
\hat{H}_{\mathrm{Q}} & =\frac{\hbar \omega_{\mathrm{Q}}}{2}\left[\hat{I}_{z}^{2} \cos ^{2} \theta-\frac{I(I+1)}{3}\right. \\
& \left.+\left(\hat{I}_{z} \hat{I}_{x}+\hat{I}_{x} \hat{I}_{z}\right) \sin \theta \cos \theta+\hat{I}_{x}^{2} \sin ^{2} \theta\right]
\end{aligned}
$$

In zero magnetic field, the $(2 I+1)$ spin levels are split according to the square of their angular momentum projection $m_{z^{\prime}}$ onto $z^{\prime}$. For a half-integer spin $I$, this leads to pairs of levels $m_{z^{\prime}}= \pm 1 / 2, \pm 3 / 2 \ldots \pm I$, which are separated from each other by $1,2, \ldots(I-1 / 2) \times \hbar \omega_{\mathrm{Q}}$, see Fig.7(a).

Although the quadrupolar interaction is qualitatively different from a magnetic field, for the purpose of quantitative comparison it is useful to express $\hbar \omega_{\mathrm{Q}}$ as an effective field $B_{\mathrm{Q}}$, where $g_{N} \mu_{N} B_{\mathrm{Q}} \equiv \hbar \omega_{\mathrm{Q}}$. In self-assembled InAs QDs estimated values of $B_{\mathrm{Q}}$ fall in the $100 \mathrm{mT}$ range. (Dzhioev and Korenev, 2007, Krebs et al., 2008, Maletinsky et al., 2009). As a result, the dipolar coupling between nuclear states of angular momentum difference $\left|\Delta m_{z^{\prime}}\right|=1$ or $\left|\Delta m_{z^{\prime}}\right|=2$ is strongly inhibited. Only the states $m_{z^{\prime}}= \pm 1 / 2$ still experience the local small fluctuating field due to surrounding nuclear spins in the order of $\delta B_{L} \approx 0.15 \mathrm{mT}$ in GaAs. The polarization relaxation induced by the non-secular part of the dipolar interaction is then essentially suppressed for the levels $\left|m_{z^{\prime}}\right|>1 / 2$ in agreement with the substantial Overhauser shift observed in QDs for zero external field, as discussed in section V.B.5. The quadrupolar shifts also lead to an energy mismatch between nuclear spin levels of atoms inside the dot compared to atoms in the surrounding barrier material which leads to a strong suppression of nuclear spin diffusion from the dot towards the barrier. As a result the mesoscopic spin system of $\approx 10^{5}$ nuclear spins in a highly strained QD like InAs in GaAs is well isolated from its surroundings.

The inclination $\theta$ of the quadrupolar axis induces oscillations of the nuclear polarization component perpendicular to $z^{\prime}$, while keeping constant the longitudinal projection along $z^{\prime}$. Under cw optical excitations this transverse part vanishes and the nuclear polarization created in a quantum dot along the external field direction $z$ is reduced by $\sim \cos ^{2} \theta$, as shown in Fig. 7(b) for nuclear spins $I=3 / 2$ and $I=9 / 2$. This effect which has to be averaged over the angle dispersion of $z^{\prime}$ may contribute to the enhancement of the effective nuclear spin relaxation observed experimentally in low magnetic fields, see Sec.V.B.3.

\section{DYNAMICS OF ELECTRON SPINS COUPLED TO A FLUCTUATING NUCLEAR FIELD}

In bulk semiconductor or quantum well structures, the electron spin dephasing induced by the interaction with nuclear spins is usually much weaker than the well-known mechanisms originating from spin-orbit interactions, well documented in Meier and Zakharchenya (1984) and Dyakonov (2008). Due to the absence of translational carrier motion in semiconductor QDs, the discrete energy levels due to carrier localization and the corresponding lack of energy dispersion lead to a strong suppression of these well-known electron spin relaxation processes (Burkard et al. 1999; Khaetskii and Nazarov, 2000, Paillard et al., 2001). The spin relaxation time due to hyperfine interaction with lattice nuclei was first derived by Dyakonov and Perel for donor-bound electrons (Dyakonov and Perel., 1973, 1974, Dzhioev et al., 2002, Paget et al., 1977) and subsequently in great detail for electrons confined to QDs (Burkard et al. 1999 , Khaetskii et al. 2002, Merkulov et al., 2002, Semenov and Kim, 2003). We review in this section the experimental and theoretical work on this topic. Throughout this section the mean nuclear spin polarization $\left\langle B_{n}\right\rangle$ is taken to be zero, i.e. no dynamic nuclear polarization (DNP) is created, see sections V/and VI for a detailed discussion about build-up, manipulation and decay of nuclear spin polarization.

\section{A. The Merkulov-Efros-Rosen Model}

Three distinct time scales are relevant for describing the electron-nuclei spin system evolution in a QD according to the Merkulov-Efros-Rosen (MER) model (Merkulov et al., 2002):

(1) the first time corresponds to the electron-spin precession around the frozen nuclear field fluctuations given by $\delta \boldsymbol{B}_{\boldsymbol{n}}$ (Burkard et al., 1999, Khaetskii et al., 2002, Semenov and Kim, 2003): the typical dephasing time is of the order of $T_{\Delta} \approx \frac{h}{g \mu_{B} \delta B_{n}} \sim 1$ ns for InAs QDs containing $10^{5}$ nuclei (see Fig. 8 ).

(2) The second time is controlled by nuclear-spin precession in the inhomogeneous hyperfine field of the localized electron (Knight field $\boldsymbol{B}_{\boldsymbol{K}}$ ): the typical time is given by $T_{K \Delta} \simeq \sqrt{N} T_{\Delta}$ which results for $N=10^{5}$ in $T_{K \Delta} \sim 1 \mu \mathrm{s}$.

(3) The third time is given by the nuclear-spin relaxation due to dipole-dipole interaction with nuclei in the vicinity of the QDs : its order of magnitude is given by the average precession time of a nuclear spin in the local field fluctuation $\delta \boldsymbol{B}_{\boldsymbol{L}}$, occurring on a typical timescale $T_{\text {Dipole }} \sim 100 \mu \mathrm{s}$.

During the first two stages, the total angular magnetic moment of an electron and the nuclei interacting with this particular electron is conserved. Thus the global coherence of the electron-nuclear spin system is preserved, while during the last stage it is not, since the dipolar 


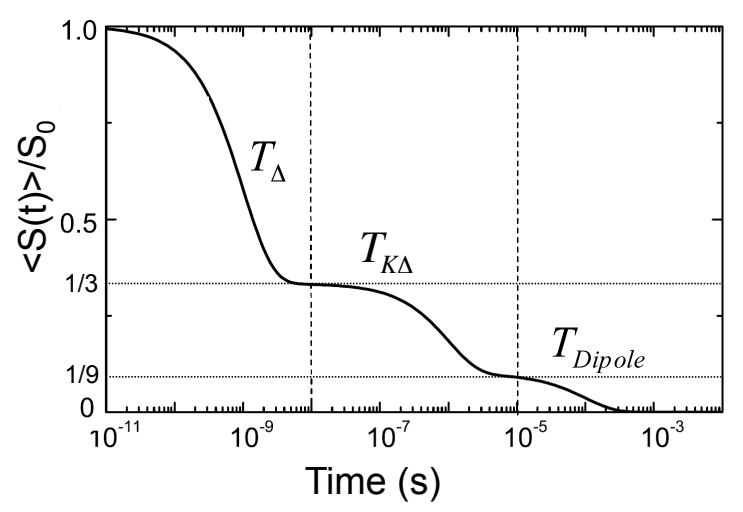

FIG. 8 Electron spin relaxation induced by nuclei in a QD. The calculation is done for $N=10^{5}$ nuclei per dot (after Chapt. 11 in Dyakonov (2008)).

interaction does not conserve the total angular magnetic moment (see Ch.VIII.E of Abragam (1961)).

Let us focus first on the shortest decay time corresponding to the electron-spin precession around the frozen nuclear field fluctuations. An electron spin in a QD interacts with a large but finite number of nuclei for instance $N \sim 10^{5}$ in an InAs QD. In the frozen fluctuation model, the sum over the interacting nuclear spins gives rise to an average local effective hyperfine field $\boldsymbol{B}_{\boldsymbol{n}}$ with a fluctuation characterized by $\delta \boldsymbol{B}_{\boldsymbol{n}}$. The dispersion of the nuclear hyperfine field $\boldsymbol{B}_{\boldsymbol{n}}$ in the absence of dynamic nuclear polarization can be described by a Gaussian distribution $W\left(B_{n}\right) \propto \exp \left(-3 B_{n}^{2} / 2 \delta B_{n}^{2}\right) . W\left(B_{n}\right)$ has a spherical symmetry indicating that $\boldsymbol{B}_{\boldsymbol{n}}$ has no preferred spatial orientation. To estimate the magnitude of $\delta B_{n}$, consider a QD made of $N$ identical nuclear spins $I$. The average amplitude of the fluctuating hyperfine field reads $\delta B_{n}=\frac{1}{g_{e} \mu_{B}} \frac{2 \tilde{A}}{\sqrt{N}} \sqrt{I(I+1)}$. The maximum Overhauser field corresponding to a $100 \%$ nuclear spin polarization is $B_{n}^{\max }=\frac{1}{g_{e} \mu_{B}} 2 \tilde{A} I$ in the order of several Tesla in InAs and GaAs. As a result $\delta B_{n} \sim B_{n}^{\max } / \sqrt{N}$ which corresponds to an effective field in InAs dots of typically $30 \mathrm{mT}$ which arbitrarily changes orientation on a time scale of $10^{-4} \mathrm{~s}$ (Merkulov et al., 2002). The exact value of $\delta B_{n}$ extracted from measurements in InAs/GaAs QDs varies typically from 20 to $40 \mathrm{mT}$. This variation has two main origins:

(i) The exact value of the hyperfine constant $\tilde{A}$ and the relevant nuclear spin $I$ depends on the exact chemical composition of the dot influenced, for example, by Gallium inter diffusion into nominally pure InAs dots, resulting in reality in $\mathrm{In}_{1-x} \mathrm{Ga}_{x}$ As dot formation.

(ii) The exact QD dimensions and hence the number of nuclei $\mathrm{N}$ interacting with the electron spin vary from dot to dot even within the same sample wafer.

Although an electron spin precesses coherently around $\delta \boldsymbol{B}_{\boldsymbol{n}}$ in a given dot, the amplitude and the direction of this effective nuclear field vary strongly from dot to dot. The average electron spin $\langle\mathbf{S}(t)\rangle$ in an ensemble of dots will thus decay during this first stage as a consequence of the random distribution of the local nuclear effective field (Braun et al. 2005; Khaetskii et al. 2002; Merkulov et al. 2002):

$$
\langle\mathbf{S}(t)\rangle=\frac{\mathbf{S}_{0}}{3}\left\{1+2\left[1-2\left(\frac{t}{2 T_{\Delta}}\right)^{2}\right] \exp \left[-\left(\frac{t}{2 T_{\Delta}}\right)^{2}\right]\right\}
$$

where $S_{0}$ is the initial spin and

$$
T_{\Delta}=\hbar\left(\frac{3 N}{2 n \sum_{j=1}^{n} I^{j}\left(I^{j}+1\right)\left(A^{j}\right)^{2}}\right)^{1 / 2}
$$

is the dephasing time due to the random electron precession frequencies in the randomly distributed frozen fluctuation of the nuclear hyperfine field; here $n$ is the number of atoms per unit cell of the lattice and the in$\operatorname{dex} j$ runs over the nuclei of a unit cell. The fluctuating field $\delta \boldsymbol{B}_{\boldsymbol{n}}$ is assumed to be isotropic for electron spin dynamics. If the electron spin is initially orientated along the $\mathrm{z}$ axis, only the components of $\delta \boldsymbol{B}_{\boldsymbol{n}}$ in the $\mathrm{x}$ and $\mathrm{y}$ directions will contribute to spin dephasing (see Fig.9.(b)). Hence only $2 / 3$ of the initial electron spin polarization is lost. Figure 8 shows that the calculated average electron spin $\langle\mathbf{S}(t)\rangle$ drops down to about $1 / 3$ of its initial value on a characteristic time $T_{\Delta} \sim 1 \mathrm{~ns}$.

The second stage of spin relaxation, occurring on a characteristic time $T_{K \Delta}$, is due to the Larmor precession of the nuclear spins in the inhomogeneous Knight field due to the electron spin (see Eq. (5)). This precession results in a new configuration of the random nuclear field. During this second stage:

$$
\langle\boldsymbol{S}(t)\rangle=\frac{\boldsymbol{S}_{0}}{3}\left\langle\boldsymbol{B}_{n}(t) \cdot \boldsymbol{B}_{n}(0)\right\rangle_{N} /\left\langle\boldsymbol{B}_{n}^{2}(0)\right\rangle_{N}
$$

where the time origin is set here at a delay of the or-

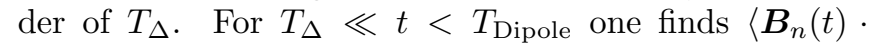
$\left.\boldsymbol{B}_{n}(0)\right\rangle_{N} /\left\langle\boldsymbol{B}_{n}^{2}(0)\right\rangle_{N} \sim 1 / 3$, since only the components $\left\langle\hat{I}_{x}\right\rangle$ and $\left\langle\hat{I}_{y}\right\rangle$ of the nuclear field precess about $\left\langle S_{z}^{e}\right\rangle \vec{e}_{z}$. The average electron spin evolves like the nuclear field correlation function (Merkulov et al., 2002$)$. The time $T_{K \Delta}$ is much longer than $T_{\Delta}$ because the interaction of an electron spin with a given nucleus is $\sqrt{N}$ times weaker compared to the interaction with the effective magnetic field of the nuclear fluctuations: $T_{K \Delta} \sim T_{\Delta} \cdot \sqrt{N} \sim 1 \mu \mathrm{s}$. During this second stage the electron spin feels a slow variation of the effective nuclear field yielding again a decrease of the average electron spin down to $1 / 9$ of its initial value $\langle\boldsymbol{S}(t)\rangle=\frac{\boldsymbol{S}_{0}}{9}$, as indicated in Fig. 8.

Finally the third stage of electron spin relaxation, labelled $T_{\text {Dipole }} \sim 100 \mu \mathrm{s}$, is due to the dipole-dipole interaction of nuclear spins. In contrast to the two first stages, this dipole-dipole interaction does not conserve the total nuclear spin and is thus the upper limit for the electron spin coherence $T_{2}$. Whereas the electron spin 

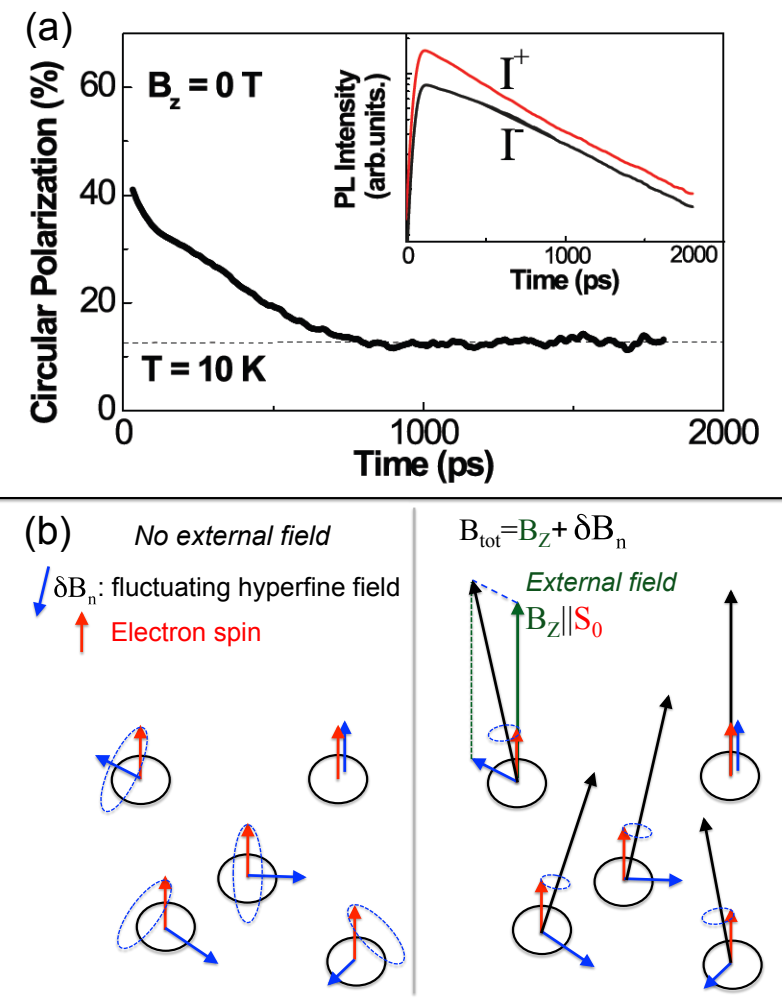

FIG. 9 (a) Circular polarization dynamics of the positively charged exciton $X^{+}$luminescence for $B=0$ for an ensemble of InAs dots. $X^{+}$is formed by a photocreated electron-hole pair and a resident hole. Since unpolarized holes form a zero spin singlet, the spin-polarized electron is not coupled to the holes by the exchange interaction, which can dominate the hyperfine interaction (Braun et al., 2005, Dyakonov, 2008). The inset displays the $I^{+}$and $I^{-}$luminescence dynamics. (b) Schematics of the electron spin precession around (left) the effective nuclear field $\delta B_{n}$ and (right) the total field $B_{t o t}=$ $B_{z}+\delta B_{n}$ if an external magnetic field $B_{z}$ is applied.

dephasing time in the fluctuating nuclear field $\left(T_{\Delta}\right)$ has been measured by different groups (see the next section), the two other stages occurring on much longer timescales are still under discussion (Fras et al., 2011). Using a terminology associated to quantum coherence, the $T_{\Delta}$ time is sometimes referred to as an inhomogeneous electron spin dephasing time.

It is important to note that the nuclear spin dynamics predicted during the second and third stage of the Merkulov model will be strongly influenced by inhomogeneous quadrupolar coupling of the nuclear spins with local electric field gradients, present in InAs QDs due to local anisotropic strain, as introduced earlier in section III.B. In practice, the strong quadrupolar effects in
InAs quantum dots (Flisinski et al., 2010, Maletinsky et al. 2009) will prevent the nuclei from precessing around the Knight field and also suppress dipolar relaxation for $B_{z}<B_{Q}$. As a result, for QD systems with strong nuclear quadrupolar coupling the description by the Merkulov model of the first stage of the electron spin relaxation in the frozen average nuclear field fluctuations on a timescale $T_{\Delta}$ is by far the most relevant of the three.

\section{B. Experimental studies of electron spin dephasing in zero external magnetic field}

In optical experiments performed on undoped QDs the photogenerated electron feels a strong effective magnetic field due to the exchange interaction with the photogenerated hole in the neutral exciton $X^{0}$ (Bayer et al., 2002, Sénès et al. , 2005). This exchange field (with a characteristic energy $\delta_{0}$ of typically hundreds of $\mu \mathrm{eV}$ ) is much stronger than the effective field due to the fluctuating nuclear field $\delta B_{n}$ of the nuclei, which thus plays a negligible role for the spin dynamics of the electron in $X^{0}$ (Erlingsson et al. 2001) in most experiments (see Stevenson et al. (2011) for experiments investigating electron dephasing due to $\delta B_{n}$ in neutral QDs). The positively charged excitons $X^{+}$, consisting of one electron and two holes forming a spin singlet, is the ideal configuration to probe the electron spin relaxation mediated by nuclei in QDs with optical experiments. Due to Kramer's theorem the anisotropic contribution to the exchange interaction between the electron and the two holes does not lead to any fine structure splitting of the $X^{+}$ground state. Thus the analysis of the circular polarization $\rho_{c}$ of the $X^{+}$luminescence in p-doped QDs following a circularly polarized laser excitation probes directly the spin polarization of the electron as $\left\langle S_{z}^{e}\right\rangle=-\rho_{c} / 2$.

Figure 9 (a) displays the circular polarization dynamics of the $X^{+}$photoluminescence from an ensemble of InAs/GaAs QDs (Braun et al. 2005). The inset shows the time evolution of the polarised luminescence intensity components. The circular polarization dynamics in Fig.9(a) presents two regimes: The polarization decays within the first 800 ps down to about $1 / 3$ of its initial value; then it remains stable with no measurable decay on the radiative life-time scale. The observed electron spin relaxation is due to the hyperfine interaction with the nuclei (Merkulov et al. 2002) : from Eq. (11), we calculate $T_{\Delta} \sim 0.5 \mathrm{~ns}$, in agreement with the observed decay time in Fig.9. (a). This corresponds to a dispersion of the nuclear hyperfine field distribution $\delta B_{n} \sim 45 \mathrm{mT}$. The subsequent electron spin dephasing $T_{K \Delta}$, which is the result of the variations of the random nuclear field direction, occurs on a time scale typically 100 times longer than $T_{\Delta}$. Thus it cannot be observed on the $X^{+}$radiative lifetime scale $(\sim 1 \mathrm{~ns})$ accessible in PL measurements. 


\section{Electron spin dephasing in a longitudinal magnetic field: Faraday geometry}

An external magnetic field applied along the $z$ growth axis (Faraday configuration) which adds to the nuclear field fluctuations $\delta B_{n}$, can stabilize the electron spin, which will then precess about the resulting total field $B_{\text {tot }}=B_{z}+\delta B_{n}$. This effect is sometimes referred to as the screening of $\delta B_{n}$ by the external field. $B_{z}$ must be larger than $\delta B_{n}$, to ensure that the Zeeman interaction of the electron spin with the magnetic field is stronger than the interaction with the nuclei (Merkulov et al., 2002). Figure 10 displays the circular polarization dynamics of the $X^{+}$luminescence with a magnetic field $B_{z}=100 \mathrm{mT}$; the dynamics for $B_{z}=0$ is also presented for comparison (Braun et al. 2005). Note that the Zeeman splitting energy of the electron in this weak magnetic field is about 100 times smaller than $k_{B} T$. By applying a field of $B_{z}=100 \mathrm{mT}$, the initial decay is suppressed since the total field affecting the electron spin becomes almost parallel to the initial spin direction as in Fig.9(b) (right). Let us emphasize that this does not mean the nuclear field fluctuations disappeared: they still strongly affect the $S_{x}$ and $S_{y}$ electron spin components which is a key obstacle for use of electron spin states in quantum information schemes, as shown in Sec.IV.D. This pronounced effect of the small external magnetic field observed in Fig.10 agrees well with the predicted influence of the external magnetic field on the electron spin relaxation by nuclei in InAs QDs (see inset of Fig. 10, Merkulov et al. (2002), Semenov and Kim (2003)) or in InP QDs (Pal et al. 2007). The effect observed here is similar to the suppression of the nuclear hyperfine interaction effects measured for localized electrons in lightly doped bulk n-GaAs (Colton et al. 2004, Dzhioev et al., 2002). For larger external magnetic fields the relaxation of the $z$ component of the electron spin is no longer governed by hyperfine interaction effects but by spin-orbit mechanisms mediated by phonon coupling (Khaetskii and Nazarov, 2000). Electron spin relaxation times of from milliseconds to seconds can then be measured for magnetic fields of the order of a few Tesla (Amasha et al., 2008, Elzerman et al., 2004, Kroutvar et al., 2004).

For repeated measurements on a single QD the hyperfine interaction has the same effect as for an ensemble of dots : $B_{n}$ will change orientation from one measurement to another since the signal integration times are commonly much longer than $T_{\text {Dipole }} \sim 100 \mu$ s so that the average is taken over a large number of uncorrelated nuclear spin configurations, as nicely demonstrated by Dou et al. (2011). Figure 11 presents the measurements of circular polarization on the $X^{+}$luminescence in a single InAs dot following a right circularly polarized excitation light (Braun et al. 2006a). Here, the time-integrated circular polarization appears to be limited to about $35 \%$ for $B_{z}=0$ as a result of the electron spin dephasing induced by the fluctuating nuclear field. At $B_{z}=240 \mathrm{mT}$ the circular polarization increases significantly up to $\sim 60 \%$. In

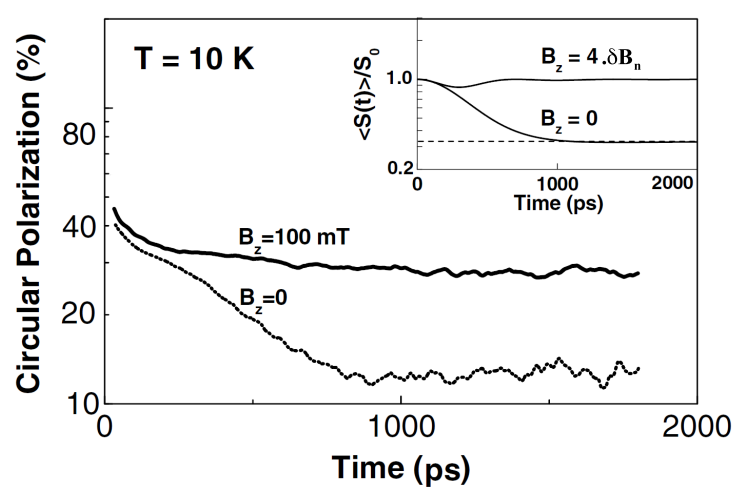

FIG. 10 Circular polarization dynamics of the $X^{+}$luminescence in an ensemble of InAs dots for $B_{z}=0, B_{z}=100 \mathrm{mT}$. The inset displays the calculated time dependence of the average electron spin $\langle\mathbf{S}(t)\rangle / S_{0}$ (Braun et al., 2005. Merkulov et al. 2002).

Fig.11(b) the experimental data show a very good agreement with the theoretical field dependence according to the MER model (Merkulov et al., 2002) which has been calculated here with $T_{\Delta}=470 \mathrm{ps}$ and an initial photogenerated electron spin polarization of the $X^{+}$state of $62 \%$. Note that the excitation polarization in this experiment is modulated at $50 \mathrm{kHz}$ between $\sigma^{+}$and $\sigma^{-}$ to prevent the dynamic polarization of nuclear spins. In the presence of DNP, the resulting mean nuclear field $\left\langle\boldsymbol{B}_{\boldsymbol{n}}\right\rangle$ would screen its own fluctuations allowing for a strong $X^{+}$circular polarization even for very weak (or even zero) external magnetic fields (Krebs et al., 2008).

A spin dephasing time $T_{\Delta} \sim 16$ ns for a resident electron in a single GaAs QD defined by monolayer fluctuations in a GaAs/AlGaAs quantum well has also been deduced from cw photominescence Hanle experiments (Bracker et al. 2005) ; this corresponds to $N \sim 5 \cdot 10^{6}$ interacting nuclei, which is consistent with the larger size of these GaAs dots (diameter $\sim 170 \mathrm{~nm}$ ) compared to the InAs QDs (diameter $\sim 20 \mathrm{~nm}$ ).

The electron spin dephasing induced by nuclei was also intensively investigated in transport experiments in gatedefined GaAs double QDs (Hanson et al. , 2007) at very low temperature $(\sim 100 \mathrm{mK})$. Rapid electrical control of the exchange interaction in gate-defined double QD devices allow the measurement of the single electron spin dynamics with an average value that decays on a characteristic time $T_{\Delta}$. The measurements show that the separated electron spins in the two QDs loose coherence in $T_{\Delta} \sim 10 \mathrm{~ns}$ (see Fig. 12. The increase of the long time saturation value of the average electron spin in a weak external magnetic field $(\sim 100 \mathrm{mT})$ is also clearly observed (Petta et al., 2005).

The electron spin dephasing induced by the nuclear field fluctuations has also been clearly evidenced in ma- 

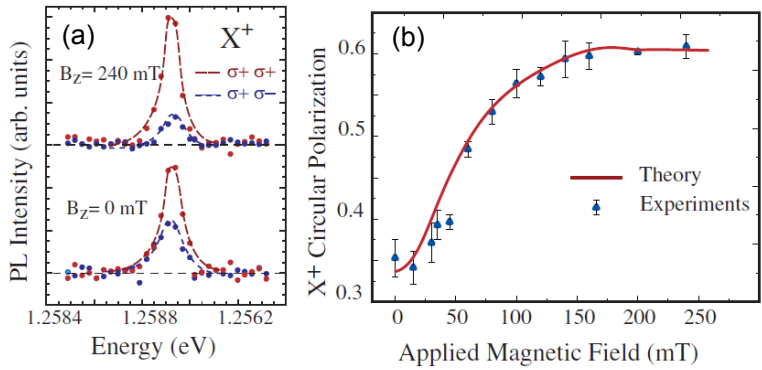

FIG. 11 Single InAs dot measurement (a) Polarizationresolved spectra of $X^{+}$luminescence for two different magnetic fields. (b) Circular polarization of $X^{+}$line versus external magnetic field $B_{z}$. The excitation polarization is provided by a $50 \mathrm{kHz}$-photo-elastic modulator to avoid the build up of a nuclear polarization through optical pumping (Braun et al. 2006a).

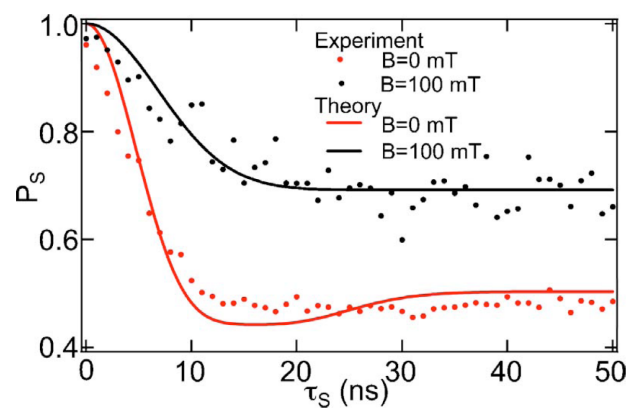

FIG. 12 Time dependence of the singlet (two-electron-spin) return probability in an electrically-defined GaAs double-QD. The data are fitted using a semi-classical model (Schulten and Wolynes, 1978) which is similar to the Merkulov-EfrosRosen approach. The fits correspond to $\delta B_{n}=2.3 \mathrm{mT}$ and a corresponding $T_{2}^{*}=10$ ns. (Petta et al., 2005).

terials where only a fraction of the nuclei has non-zero nuclear spins (in InAs or GaAs, all the nuclei have a nonzero spin) such as $\mathrm{ZnO}$ (Liu et al., 2007; Whitaker et al. 2010), diamond (Balasubramanian et al. | 2009; Childress et al. 2006) and CdSe (Akimov et al., 2006). The electron spin relaxation dynamics in colloidal n-type $\mathrm{ZnO}$ QDs has been studied using electron paramagnetic resonance spectroscopy. In $\mathrm{ZnO}$, only the isotope ${ }^{67} \mathrm{Zn}$ has a non zero nuclear spin $(\mathrm{I}=5 / 2)$ with a natural abundance of $4.1 \%$. The nuclear spin contents in the $\mathrm{ZnO}$ QDs can be controlled chemically by preparing nanocrystals from precursors containing different concentrations of ${ }^{67} \mathrm{Zn}$ (see Fig. $13(\mathrm{a})$ ). As expected, the electron spin

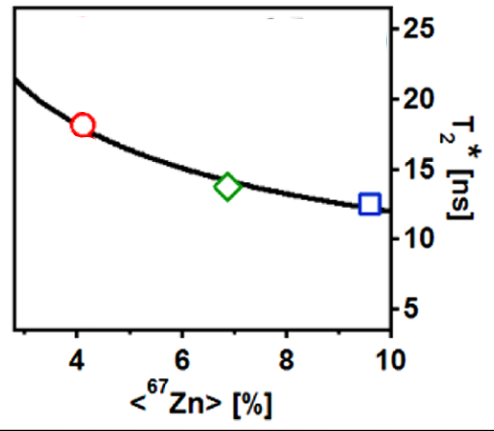

(a)

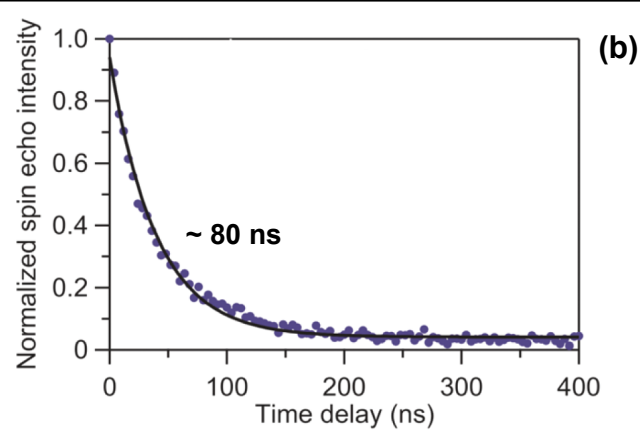

FIG. 13 (a) Electron spin dephasing time $T_{2}^{*}=T_{\Delta}$ in colloidal n-type $\mathrm{ZnO}$ nanocrystals as a function ${ }^{67} \mathrm{Zn}$ : (circle) $4.1 \%$ natural abundance, (diamond) $6.8 \%$, and (square) $9.6 \%$ ${ }^{67}$ Zn. (Liu et al. 2007) ; (b) Spin-echo decay curve measured by Electron Paramagnetic Resonance spectroscopy of $\mathrm{ZnO}$ nanocrystals $\left(d \sim 4.0 \mathrm{~nm},{ }^{67} \mathrm{Zn}=4.1 \%\right)$ at $5 \mathrm{~K}$ (Whitaker et al. 2010).

dephasing time decreases as the ${ }^{67} \mathrm{Zn}$ concentration increases. Note that this dependence was observed at room temperature.

As $T_{\Delta}$ is governed by inhomogeneous contributions of the fluctuating nuclear field, spin echo measurements should yield much longer spin coherence times. Indeed single pulse Hahn-echo techniques were successfully applied to transport measurements in a gate defined GaAs QD-molecule with a measured coherence time on the order of $30 \mu \mathrm{s}$ (Bluhm et al., 2010$)$ and also in optical studies of single InAs QDs (Press et al. 2010$)$. However this time does not correspond to a true $T_{2}$ time, since the slow time evolution of the nuclear field $\left(T_{K \Delta}\right.$, see Sec.IV.A leads to an incomplete recurrence. One origin of the inhomogeneity comes from the different precession period of the nuclear spins due to the different nuclear species in the GaAs dot $\left({ }^{69} \mathrm{Ga},{ }^{71} \mathrm{Ga},{ }^{75} \mathrm{As}\right)$. By implementing a multiple-pulse echo sequence and taking into account these different precession periods, a record coherence time of $200 \mu \mathrm{s}$ has been measured (Bluhm et al. 2010), see also an interesting theoretical discussion in this context (Cywinski et al. 2009). In ZnO, pulsed electron paramagnetic resonance measurements also yield a longer spin coherence time (spin echo decay time $\sim 80 \mathrm{~ns}$ 
for ${ }^{67} \mathrm{Zn}=4 \%$ compared to $T_{2}^{*} \sim 20 \mathrm{~ns}$ ) as displayed in Fig. 13(b). In practice, long spin coherence times reaching $T_{2}=3.0 \mu \mathrm{s}$ for individual dots in an ensemble of InAs dots, were also demonstrated for resident electrons using an original mode-locking technique (Greilich et al. 2006b) which can be described in the framework of narrowed state dynamics, see (Yao et al., 2006) for discussion.

\section{Electron spin dephasing in a transverse magnetic field: Voigt geometry}

When an external magnetic field $B_{x}$ is applied perpendicular to the initial electron spin orientation which corresponds to the excitation light propagation axis $\mathrm{z}$ (Voigt geometry), the electron spin will precess coherently around the external magnetic field axis. The damping of these oscillations reflects directly the spin dephasing time (Dutt et al., 2005). As shown in Sec.IV.B the time- and polarization resolved $X^{+}$photoluminescence signal following a circularly polarized pulsed excitation in an ensemble of p-doped InAs QDs can directly probe the electron spin dynamics during the charged exciton $X^{+}$ radiative lifetime. Figure 14 shows the damping of the PL circular polarization oscillations in a transverse magnetic field $B_{x}=750 \mathrm{mT}$. The observed damping of the oscillations due to electron spin dephasing has two origins. The first one is due to the $\delta B_{n}$ induced spin dephasing, with a characteristic dephasing time $T_{\Delta} \sim 500 \mathrm{ps}$ (Lombez et al., 2007a). This is the same value obtained in the absence of an external magnetic field and confirms that prolonging the macroscopic coherence time can not simply be achieved through application of a magnetic field. A true narrowing of the nuclear spin distribution i.e. lowering of $\delta B_{n}$ is necessary, for example via dynamic nuclear polarization, discussed in detail in Sec.V. Yet, the characteristic spin dephasing time $T_{\Delta} \sim 500 \mathrm{ps}$ is not enough to explain all experimental observations, see Fig.14 (top curve). At the origin of the second contribution lies the dispersion of the transverse electron Lande $\mathrm{g}$ factor, due to the inherent inhomogeneity of the system. This magnetic-field-dependent damping arises simply from the variations of the electron $g$ factor over the QD ensemble (Dutt et al. 2005: Greilich et al., 2006a), resulting in a spreading of the Larmor frequencies with increasing $B_{x}$ (Yugova et al. 2007 ). A typical fluctuation of $\Delta g / g=0.07$ explains the observed damping of the electron spin oscillations (see Fig.14, bottom curve) which follows the simple law:

$$
T_{2}^{*}=T_{\Delta} / \sqrt{1+2\left(\frac{\Delta g}{g} \frac{B}{\delta B_{n}}\right)^{2}}
$$

where $\delta B_{n}=\hbar / g \mu_{B} T_{\Delta}$.

The ensemble spin dephasing observed in Fig.14 does not lead to a destruction of the individual spin coherence, but masks it due to phase differences among different spins in the different dots. An elegant technique,

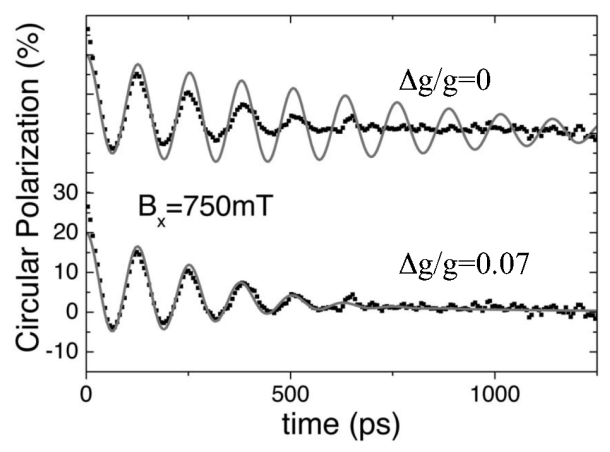

FIG. 14 Comparison of experimental (dotted line) and theoretical curves of time-resolved photoluminescence circular polarization of $X^{+}$for a transverse magnetic field $B_{x}=750 \mathrm{mT}$. The damping reflects the electron spin dephasing in the ensemble of InAs dots. The theoretical curves (gray line) is given with $\Delta g / g=0.07$ or without $\Delta g / g=0$ electron gfactor fluctuations (Lombez et al., $2007 \mathrm{a}$ ).

called mode-locking of electron spins, can be used to measure the single electron coherence time with the measurements performed in an ensemble of dots (Greilich et al. 2006b). The principle is to excite an ensemble of n-doped QDs (containing a single resident electron) with a periodic train of polarized picosecond laser pulses resonant with the $X^{-}$state and then probe the spin dynamics by Faraday rotation technique. This excitation will spin polarize the resident electrons which will precess around the transverse magnetic field $B_{x}$. The key point is that the pulse train will yield a synchronization of the electron spin precession. If the pulse period, $T_{R}$, is equal to an integer number $n$ times the electron spin precession period, the action of such pulses leads to almost complete electron spin alignment along $z$ at each pulse arrival time (see Fig.15(a)).

In the ensemble of excited QDs, the electrons do not precess with the same frequency because of the fluctuations of the electron $\mathrm{g}$ factors. In this ensemble, some QDs will have a precession frequency which fulfils this synchronization relation with the laser, termed phase synchronization condition (PSC). When a given pump pulse excites the sample the spin coherence generated by the previous pulse has the same orientation as the one which the subsequent pulse induces for these dots. In other words the contributions of all pulses in the train are constructive. In contrast, in the non-PSC dots the contributions have arbitrary orientations (see right panel of Fig.15(a)) and for these dots the degree of spin synchronization will vanish. The fraction of dots in the ensemble that fulfil the PSC will increase when nuclear spin induced frequency focussing starts to be efficient, as discussed in the follow-up work by Greilich et al. (2007), see discussion in section V.C.4 and references therein. In the experiment a PSC dot makes a stronger contribution to the Faraday rotation signal than a non-PSC dot. These 

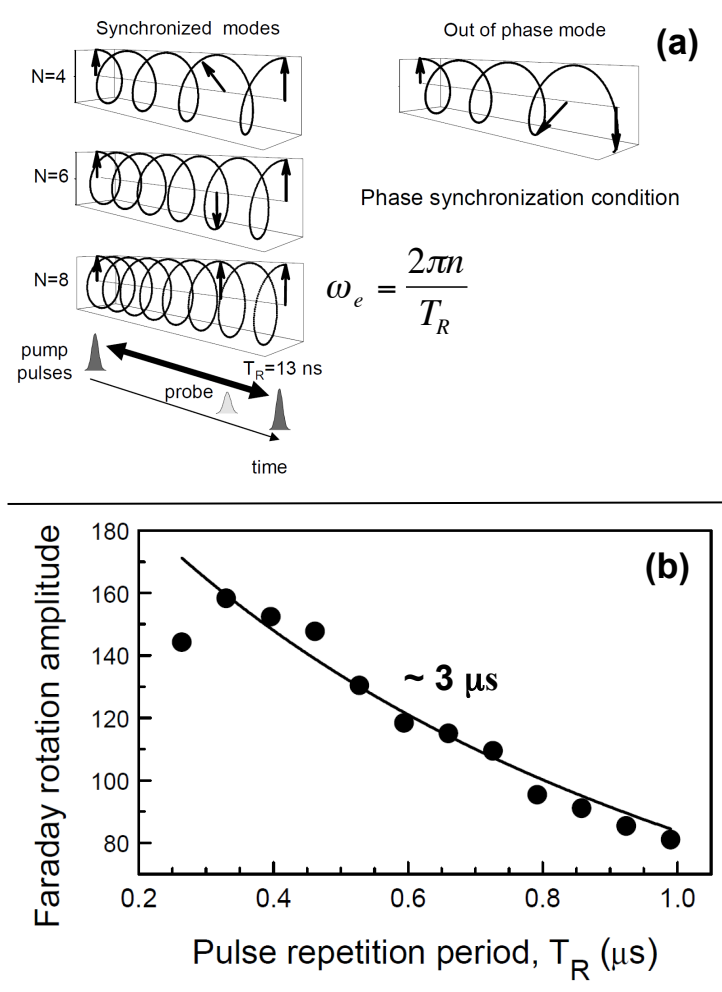

FIG. 15 (a) Schematics of the mode-locking of electron spins in an ensemble of spins precessing around a transverse magnetic field at different frequencies due to the $\mathrm{g}$ factor fluctuations from dot to dot (see text). (b) Faraday rotation amplitude at negative delay in an ensemble of n-doped InAs QDs as a function of the time interval between subsequent pump pulses measured at $B_{x}=6 \mathrm{~T}$ and $\mathrm{T}=6 \mathrm{~K}$ (Dyakonov 2008. Greilich et al. 2006b).

latter dots do not contribute to the average electron spin polarization $S_{z}(t)$ at times $t \gg T_{2}^{*}$, due to dephasing. The sum of oscillating terms from all synchronized subsets leads to a constructive interference of their contributions to the Faraday rotation signal around the times of pump pulse arrival. By measuring the Faraday signal at such delays, the synchronized spin dynamics which move on a background of dephased electrons, can be measured. When the pump pulse period becomes comparable with the electron spin coherence time, the amplitude of the signal decreases, Fig.15(b).

This yields the measurement of a spin coherence time of the order of a few $\mu s$ whereas the ensemble spin dephasing time $T_{\Delta}=0.4 \mathrm{~ns}$ at $B_{x}=6 \mathrm{~T}$. This measured value of $T_{2}^{*}$ with respect to $T_{\Delta}$ is however not limited by the spin relaxation time within one particular dot. The nuclear field fluctuations may help to achieve the phase synchronisation condition, by using $\frac{1}{\hbar}\left(g_{e} \mu_{B}\left(B_{n}+\delta B_{n}\right) T_{R}\right)=2 \pi n$. However, this condition cannot hold at times longer than the correlation time of the nuclear field fluctuations. As a consequence, the nuclear field fluctuations contribute towards limiting the spin coherence time $T_{2}^{*}$.

\section{E. Influence of the fluctuating nuclear field on electron and hole spin pumping processes}

The interaction of the localized electron spin in a quantum dot with the surrounding fluctuating nuclear spins plays an important role for the QD spin state preparation, a key issue from the perspective of quantum information processing (Imamoglu et al., 1999). High-fidelity preparation of a QD spin state via laser cooling, also referred to as optical spin pumping, has been convincingly demonstrated for electrons (Atature et al., 2006) and holes (Gerardot et al., 2008). This is at first sight surprising, given the strong influence of fluctuating nuclear fields on electron spin dephasing. But as shown below, the presence of a fluctuating nuclear field can be exploited to enable efficient carrier spin pumping processes.

Electron spin pumping.- In the experiment of Atature et al. (2006) optical coupling of electronic spin states was achieved using resonant excitation of the negatively charged exciton (trion) transition $\mathrm{X}^{-}$. A singlycharged QD is described as a four-state system consisting of twofold degenerate ground and excited states, coupled by two vertical optical transitions, as illustrated in Fig. 16(left). The ground state $|\uparrow\rangle(|\downarrow\rangle)$ is coupled to the trion state consisting of two electrons in a singlet and a heavy hole $\Uparrow(\Downarrow)$ according to the optical selection rules for $\sigma^{+}\left(\sigma^{-}\right)$optical transition. The diagonal transition between the trion state $|\uparrow \downarrow \Downarrow\rangle$ and the electron $|\uparrow\rangle$ is forbidden for a heavy hole with pure $\pm 3 / 2$ angular momentum. In reality the diagonal transition characterized by a rate $\gamma$ is permitted and the efficiency of electron spin pumping depends on the relative magnitude of $\gamma$ and $\gamma_{\mathrm{hf}}$. There are three physically distinct contributions that determine the strength of $\gamma$ : (i) Valence band mixing due to the reduced symmetry of the QD (elongation, strain anisotropy, interfaces), leading to a small light hole admixture of the heavy hole state (Belhadj et al. 2010 , Calarco et al., 2003, Krizhanovskii et al. 2005). (ii) The (unintentional) application of a transverse magnetic field (Xu et al. , 2007), arising from a slight tilt in the sample holder. (iii) In-plane component of the fluctuating Overhauser field.

In a small applied longitudinal field $B_{z} \leq \delta B_{n}$ no efficient electron spin state preparation can be achieved using resonant excitation since the strong hyperfine interaction of the resident electron spin with the QD nuclear spin ensemble leads to random spin-flip events at rate $\gamma_{\mathrm{hf}}$ (Merkulov et al. 2002), see Sec.IV.B.

As shown in Sec.IV.C, a longitudinal magnetic field exceeding $\delta B_{n}$ suppresses the effect of this interaction. As a consequence, the $|\uparrow\rangle$ state can be prepared and an absorption drop in differential transmission exper- 


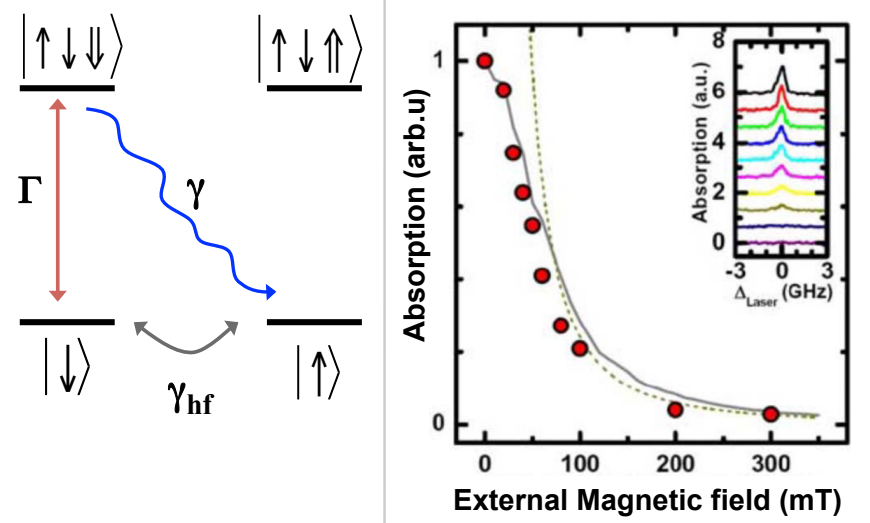

FIG. 16 Electron spin pumping in a single dot. (Left) Fourlevel system describing the singly charged QD. The fluctuations of the hyperfine field lead to a slowly varying coherent coupling of the spin ground states with a rate $\gamma_{h f}$ and the heavy hole - light hole mixing yields a diagonal transition with a rate $\gamma$ (Right) Absorption of the single dot as a function of the magnetic field $B_{z}$. The absorption decreases with increasing $B_{z}$ due to Optical Spin Pumping which becomes more efficient since the efficieny of the hyperfine field coupling of the ground state decreases. The inset shows the corresponding raw laser scans from $0 \mathrm{~T}$ (top) to $300 \mathrm{mT}$ (bottom) (Atature et al., 2006).

iments is clearly observed in Fig.16 when an external magnetic field is applied. With increasing $B_{z}$, the QD becomes transparent which is a signature of optical electron-spin pumping into the spin $|\uparrow\rangle$ state due to the weak recombination path $(\gamma)$ that dominates over the hyperfine-induced bidirectional spin-flip process with a rate $\gamma_{\mathrm{hf}}\left(B_{z}\right)$ which is decreasing as a function of the applied magnetic field (see Fig.16). For $B_{z}=200 \mathrm{mT}$, it has been shown that the electron spin can be prepared with an average polarization as high as $98.5 \%$ (Dreiser et al., 2008). In summary, the hyperfine interaction of the resident electron with the fluctuating nuclear spins prevents the realization of spin-state preparation with high fidelity in negatively charged QDs. The application of an external magnetic field is essential to achieve this spin state preparation.

Hole spin pumping. - In a positively charged QD (i.e a QD containing a resident hole), the situation is reversed as compared to the $X^{-}$case: the interaction between the electron in the positively charged exciton $X^{+}$with the nuclear spins allows coherent coupling between the two electron spin states, i.e. between $|\Uparrow \Downarrow, \downarrow\rangle$ and $|\Uparrow \Downarrow, \uparrow\rangle$ yielding an efficient hole spin cooling (Eble et al., 2009, Gerardot et al., 2008), as schematically shown in Fig. 17(a). The hole spin cooling is efficient since the rate $\gamma_{\mathrm{hf}}$ is faster than the spontaneous radiative recombination rate $\Gamma_{0}$. If a longitudinal magnetic field larger than $\delta B_{n}$ is applied, the coherent (a)
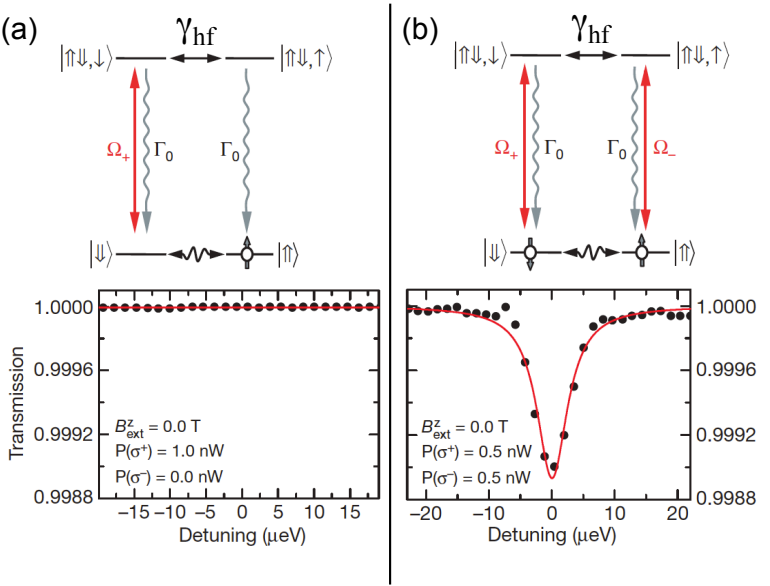

FIG. 17 Hole spin pumping in a single dot. (a) A laser with $\sigma^{+}$polarization drives the $|\Downarrow\rangle \longleftrightarrow|\Uparrow \downarrow, \downarrow\rangle$ transition. No transmission dip is observed demonstrating that the hole population is shelved into state $|\Uparrow\rangle$. (b) simultaneous excitation with both $\sigma^{+}$and $\sigma^{-}$at the same frequency. A large transmission dip is observed, as the additional $\sigma_{-}$excitation leads to a re-pumping of the electron spin and thereby suppresses electron spin pumping (Gerardot et al. 2008).

coupling between the two electron spin states within the trion is diminished and as a consequence the hole spin preparation fidelity decreases (Gerardot et al. 2008 ). Although hole spin pumping is very efficient for $B_{z}=0$, it need not be absent for $B_{z}>0$. When an exciting laser strictly resonant with the $X^{+}$transition in a single QD has circular polarization, either $\sigma^{+}$(see Fig. 17(a)) or $\sigma^{-}$, no absorption is observed, demonstrating the efficient hole spin cooling. However by pumping with two lasers with identical wavelength and with the same total power, one with $\sigma^{+}$and one with $\sigma^{-}$polarization, a clear absorption appears (Fig.17(b)). This arises because spin pumping with $\sigma^{+}$polarization is frustrated by the $\sigma^{-}$excitation, and vice-versa.

A comparison of hole and electron spin pumping is shown in Desfonds et al. (2010) which highlights that at zero applied magnetic field hole spin pumping is possible, whereas electron spin pumping is not. Both the hyperfine mediated electron and hole spin flips as well as heavy hole - light hole mixing are limiting processes for electron spin pumping, as discussed in the context of a quantumdot-spin single-photon interface in Lu et al. (2010) and Yilmaz et al. (2010).

\section{F. Beyond the nuclear mean field approach}

In order to go beyond the nuclear mean field approach developed above, powerful quantum models have been developed (Khaetskii et al. 2003, Zhang et al., 2006). G. G. Kozlov proposed a full quantum model for describing the time evolution of a single electron spin $S=1 / 2$ in- 
teracting with an even number of nuclei $2 \mathrm{~N}\left(\mathrm{~N} \approx 10^{3}\right)$ in a single QD (Kozlov, 2007). The main assumptions of this model are: (i) the electron envelope function is considered as constant within the dot; (ii) The nuclear spin interacting with the electron are identical and possess an angular momentum $I^{j}=1 / 2$. The first assumption allows the use of the total angular momentum of the QD nuclei to classify their spin states. At zero external magnetic field, the solution of the coupled system evolution problem is analytical, and the time dependence of the electron average spin $\left\langle\hat{S}_{z}(t)\right\rangle$ is then identical to the one obtained in the Merkulov model, see Eq. 12 . It thus reproduces quantitatively the fast relaxation of the electron spin observed experimentally just after the pulsed laser excitation of an individual dot (Dou et al., 2011). This is in contrast to measurements on ensembles, where this initial dip is usually masked due to variations of this particular electron spin relaxation time from dot to dot. Some fundamental differences exist between the quantum and the effective field model (Petrov et al., 2009). In the mean field approach, coherences between the electron spin states arise if one considers a single dot excited with a single pulse i.e. an electron spin precesses coherently around $\delta B_{n}$. These coherences will disappear in the averaging process of repeated laser pulses, when a sufficiently high number of different nuclear field configurations have been probed within the measurement process. On the other hand, in the microscopic model, coherences arise only within the coupled electron-nuclear spin system, which becomes fully entangled as time increases. As a consequence, it can be shown that no spin coherences can develop in the electron subsystem (Petrov et al. 2009). The spin populations are then evaluated through repeated measurements.

\section{OPTICAL PUMPING OF NUCLEAR SPINS}

Starting in sec.II.E we have described experiments that allow preparing an electron spin state with a suitable laser pulse. In this section we explore under which conditions the electron spin polarization can be transferred to the nuclear spin ensemble in the QD.

As discussed in Sec.IV.A the hyperfine interaction leads to electron spin dephasing within a characteristic time $T_{\Delta} \sim 1$ ns due to the statistical distribution of nuclear Overhauser field $\boldsymbol{B}_{n}$ in a QD ensemble (Merkulov et al. 2002). This effect is directly evidenced in the time domain for an ensemble of positively charged QDs by monitoring the PL polarization decay of $X^{+}$trions shown in Fig.9 (Braun et al. 2005). Since the correlation time $T_{2}$ of $\boldsymbol{B}_{n}$ amounts to $\approx 10^{-4} \mathrm{~s}$, this spin dephasing also manifests itself when repeatedly recording the emission stemming from a single QD for a signal integration time $\tau_{i} \gg T_{2}$. Under such conditions, the time-integrated circular polarization of a single $X^{+}$line excited by circularly
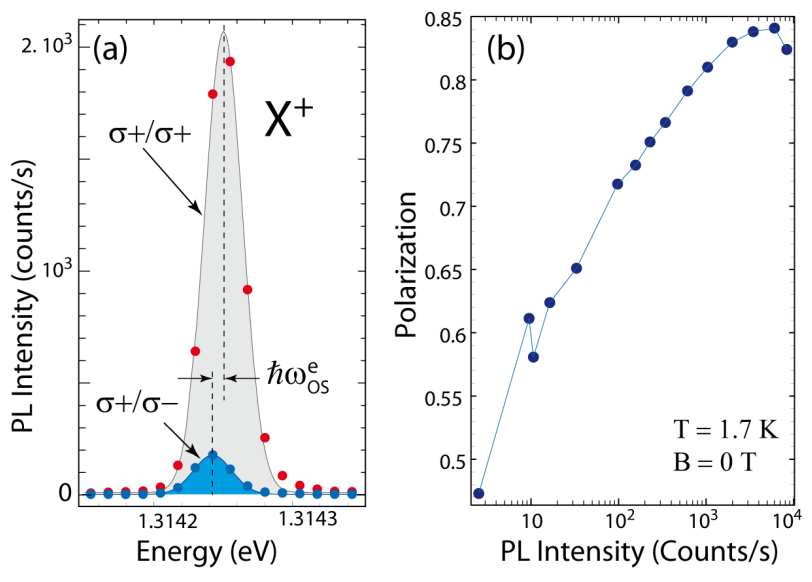

FIG. 18 (a) PL spectra of a single $X^{+}$line in zero magnetic field measured under co-polarized $\left(\sigma^{+} / \sigma^{+}\right)$and crosspolarized $\left(\sigma^{+} / \sigma^{-}\right)$excitation/detection configuration. (b) Evolution of the corresponding circular polarization as a function of the total PL intensity for an incident laser excitation varied from $150 \mathrm{nW}$ to $250 \mu \mathrm{W}$.

polarized light reads:

$$
\rho_{c}=2 \Gamma \int\left\langle S_{z}(t)\right\rangle \exp (-\Gamma t) d t
$$

where $\left\langle S_{z}(t)\right\rangle$ is the electron spin evolution averaged over the distribution of random nuclear fields and $\Gamma=1 / \tau_{r}$ is the $X^{+}$recombination rate. Using Eq. 12 from Sec.IV.A for $\left\langle S_{z}(t)\right\rangle$ with a nuclear field fluctuation of $\delta \overline{B_{n} \approx}$ $30 \mathrm{mT}$, the maximum degree of polarization for an initially photo-created spin $S_{z}(0)=1 / 2$ is estimated to be $\rho_{c}^{\max } \sim 50 \%$. However, this limit is commonly exceeded in experiments measuring the PL polarization of single $X^{+}$line under cw excitation for signal integration times $\tau_{i} \sim 1 \mathrm{~s}$ (Ebbens et al. 2005 ; Eble et al., 2006; Lai et al. 2006). This is illustrated in Fig. 18 (a) and (b) showing that the circular polarization of a single $X^{+}$line reaches more than $80 \%$ under quasi-resonant excitation. This apparent discrepancy turns out to be the manifestation of efficient DNP acting back on the electron spin dynamics. The power-dependence of the circular polarization shown in Fig. 18(b) supports this interpretation: at very low excitation density the $X^{+}$circular polarization reduces to $\approx 50 \%$ because the average nuclear spin polarization vanishes. The experimental approach is often as follows: The electron spin is initialised via optical pumping that induces DNP. The back action of the nuclear spin system on the electron is then monitored by recording the resulting changes in electron spin orientation and transitions energy, revealing the surprising bistability, memory and line dragging effects detailed below.

\section{A. Dynamic Nuclear Polarization : general features}

The dynamic nuclear polarization in semiconductor quantum dots results from the scalar form $\hat{\boldsymbol{S}}^{e} \cdot \hat{\boldsymbol{I}}$ of the hy- 
perfine interaction which conserves the total spin. When an electron spin relaxes its initial orientation via this interaction, its spin angular momentum is transferred to the nuclear spins. This corresponds to the electron-nuclei flip-flop term in Eq. (6). To achieve a significant nuclear polarization via the Overhauser effect (Overhauser, 1953) it is required to maintain the spin polarization of unpaired electrons far from their equilibrium value $\left\langle S_{z}^{e}\right\rangle_{0}$ which is usually determined by the lattice temperature $T$ and the external magnetic field $B_{z}$. The trend of electrons to return to their thermal equilibrium populations leads to an increase of the nuclear spin polarization via the spin flip channel provided by the hyperfine interaction. Since the nuclei are themselves much less coupled to the lattice, their polarization relaxes on a much longer timescale than electron polarization and a large DNP degree can be reached. The relation between the DNP build-up time and its relaxation time will be discussed more closely in the following sections.

In many experiments the out-of-equilibrium electron spin polarization is achieved by saturating the electron spin resonance by an RF field to achieve equal populations of spin $\uparrow$ and $\downarrow$ in a non-zero magnetic field. Yet, in semiconductors and more specifically in QDs, a large electron spin polarization can be created optically, even in zero magnetic field, due to the selection rules permitting excitation of specific spin states with the light polarization as discussed in Sect.II.E. The very efficient optical orientation of electrons in p-type InAs QDs is therefore well suited for generation of a large DNP via the Overhauser effect. Interestingly, the polarization-resolved PL spectroscopy of such individual QDs provides an access to both the electron spin component $\left\langle S_{z}^{e}\right\rangle$ via the circular polarization, and the average nuclear field $B_{n, z}$ via the energy splitting of the $\sigma^{+}$and $\sigma^{-}$components. In Fig. 18(a) for $B_{z}=0$, the $\sigma^{+}$PL line is clearly blue-shifted from the $\sigma^{-}$line by $\hbar \omega_{O S}^{e}=7 \mu \mathrm{eV}$. This corresponds to an average nuclear field $B_{n, z}=\hbar \omega_{O S}^{e} /\left(g_{e} \mu_{\mathrm{B}}\right)=-200 \mathrm{mT}$ which is notably larger than its own fluctuations $\delta B_{n}$. In this experiment, a stationary regime is thus established, where the relaxation by the hyperfine interaction is efficiently quenched due to the nuclear field it has itself established during some initial DNP transient.

The spin flip-flops permitted by the hyperfine interaction are however not energy-conserving in a finite external magnetic field. Electron and nuclear spins have indeed very different Landé factors yielding a ratio of their Larmor frequencies $\omega_{Z}^{n} / \omega_{Z}^{e} \sim 10^{-3}$, so that the electron Zeeman energy $\hbar \omega_{Z}^{e}$ has to be exchanged with some other degrees of freedom. This is the reason why the coupling of the electron to a reservoir is in general important for the generation of a sizeable Overhauser field: it provides a finite width (given by a finite correlation time $\tau_{c}^{e}$ ) for the electronic levels necessary to account for the energy cost of flip-flops, see (Baugh et al., 2007) for a related discussion ${ }^{3}$. In QDs, this issue of energy conservation is essential for explaining the emergence of strong nonlinearities of the electron-nuclei system that will be discussed in the next sections.

A different mechanism for DNP called 'solid-state effect' was first observed in crystals with paramagnetic impurities (Abragam, 1961). Here the energy necessary for a spin flip-flop is provided by an external RF source. In close analogy in optical pumping experiments the energy necessary for a spin flip-flop can be provided by a driving laser, in experiments similar to the solid-state effect carried out by Chekhovich et al. (2010a), where a resonant laser drives an optical transitions that is only weakly allowed due to hyperfine coupling, see Sec.V.C.

In general, the specific conditions of QD optical excitation (e.g. strictly- or quasi-resonant, with our without applied magnetic fields) determine the DNP mechanism that dominates and hence controls the magnitude and sign of the nuclear field experienced by electrons in the QD volume. If the electron spin coherence time resulting from optical excitation is short, then the flip-flop term $\propto\left(\hat{I}_{+}^{j} \hat{S}_{-}^{e}+\hat{I}_{-}^{j} \hat{S}_{+}^{e}\right)$ of Eq. 6 introduced in Sec. III.A will be the dominant nuclear spin pumping mechanism; this is typically the case for quasi-resonant or non-resonant excitation. In strong magnetic fields and under resonant excitation of the fundamental exciton or trion resonances on the other hand, the electron spin flip is too costly in energy and nuclear spin flips can be induced by two additional forms of electron-nuclear spin coupling, that do not require a simultaneous electron spin flip:

(i) The nuclear spin dynamics in strained QDs will also be influenced by strong nuclear quadrupole effects, introduced in Sec.III.B, giving rise to a non-collinear coupling term, which we will use in the following simplified form (Huang and $\mathrm{Hu}, 2010$ ):

$$
\hat{H}_{h f}^{n c}=\sum_{i} A_{\mathrm{nc}}^{i} \hat{I}_{\mathrm{x}}^{i} \hat{S}_{\mathrm{z}}^{e}
$$

with a non-collinear hyperfine interaction constant $A_{\mathrm{nc}}$ that is typically $A_{\mathrm{nc}} \approx 0.01 A$. The physical origins of this coupling are detailed in Sec.V.C.3. $\hat{H}_{h f}^{n c}$ can induce under certain conditions nuclear spin relaxation, as detailed in Sec.V.B.2. Interestingly, this term is also at the origin of bi-directional DNP and explains the experimentally observed locking of a QD transition to a resonant

\footnotetext{
3 There are several physical mechanisms that will influence the correlation time, for example any physical process that limits the electron spin lifetime. Following quasi-resonant or non-resonant optical excitation, the spin flip-flop with a nucleus can take place while the electron is in an excited state in the QD, leading to $\tau_{c}^{e}$ in the tens of ps range, or for an electron in the QD ground state, so $\tau_{c}^{e}$ is limited by the radiative lifetime $\tau_{r} \simeq 1 \mathrm{~ns}$. The electron spin lifetime could also be limited by co-tunneling events in charge tunable structures. In addition, the hyperfine induced flip-flop events themselves will limit the correlation time.
} 
driving laser ('dragging') (Högele et al., 2012, Yang and Sham, 2010) described in Sec.V.C.3.

(ii) Even as direct electron-nuclear spin-flip processes are forbidden by energy conservation at high magnetic fields, elimination of the flip-flop terms of Eq.6. using a Schrieffer-Wolff transformation (Schrieffer and Wolff, 1966) shows that the QD electron can still strongly influence the nuclear dynamics: in addition to the nuclear dipole-dipole interaction given by Eq.7, nuclear spins can also be coupled to each other by an indirect interaction mediated by the electron in the dot which has the form Abragam, 1961: Klauser et al. 2006):

$$
\hat{H}_{i n d}=\sum_{i, j} \frac{A^{i} A^{j}}{\omega_{Z}^{e}} \hat{S}_{z} \hat{I}_{+}^{i} \hat{I}_{-}^{j} .
$$

This Hamiltonian ensures the conservation of the total nuclear spin polarization but leads to nuclear spin diffusion (Latta et al. 2011b), as discussed in Sec.VI.C. Indirect coupling of nuclear spins causing spin relaxation is also induced in charge tunable structures (Warburton et al. 2000) through electron co-tunneling (Dreiser et al. 2008 Smith et al. 2005) introduced in Sec.II.E.

It is the intricate balance between these different nuclear spin pumping and depolarization mechanisms that gives rise to the intriguing experimental findings detailed below.

\section{B. Dynamic nuclear polarization in single quantum dot optics}

\section{Nonlinearity of the dynamic nuclear polarization}

The very first observations of nuclear polarization in InAs quantum dots (Braun et al., 2006b Eble et al. 2006 Maletinsky et al., 2007b Tartakovskii et al., 2007) have revealed a striking asymmetry of the magnitude of the generated Overhauser field $\boldsymbol{B}_{n}$ as a function of the light helicity $\sigma^{+}$or $\sigma^{-}$used for excitation when a magnetic field $\boldsymbol{B}$ of only a few $100 \mathrm{mT}$ is applied parallel to the optical axis (Faraday configuration) ${ }^{4}$. The general expression for $\boldsymbol{B}_{n}$ due to the Overhauser effect in bulk semiconductors is however essentially symmetrical with respect to the electron spin direction (Meier and Zakharchenya, 1984) :

$$
\boldsymbol{B}_{n}=b_{n} \frac{\boldsymbol{B}\left(\boldsymbol{B} \cdot \boldsymbol{S}^{e}\right)}{\boldsymbol{B}^{2}+\xi B_{L}^{2}}
$$

Here $b_{n}$ is a proportionality constant and $B_{L} \sim 0.15 \mathrm{mT}$ is the small local effective magnetic field experienced by the nuclei due to their mutual dipole-dipole interaction and $\xi$ is a coefficient close to unity. Note that

\footnotetext{
4 This asymmetry was not observed in the well characterised GaAs interface fluctuation dots (Gammon et al. 2001).
}

this expression assumes that a nuclear spin temperature exists ${ }^{5}$. This expression is valid for moderate fields where the equilibrium spin polarization $\left\langle S_{z}^{e}\right\rangle_{0}=$ $1 / 2 \tanh \left(g_{e} \mu_{\mathrm{B}} B_{z} / k_{\mathrm{B}} T\right)$ can be neglected. For magnetic fields below $\sim 10 \mathrm{mT}$, the Knight field $\boldsymbol{B}_{K} \propto\left\langle\boldsymbol{S}^{e}\right\rangle$ should also be added to $\boldsymbol{B}$ in Eq. (17). This introduces an asymmetry which however vanishes for fields above $100 \mathrm{mT}$. Also the strong nuclear quadrupole effects in strained QDs introduced in Sec.III.B need to be included when analysing the magnitude and orientation of $\boldsymbol{B}_{n}$.

To establish an expression similar to Eq. (17) valid in the case of QDs where large nuclear fields are generated, the dependence of the electron-nuclei flip-flop rate (hidden in $b_{n}$ ) on the total magnetic field $\boldsymbol{B}+\boldsymbol{B}_{n}$ has to be taken into account explicitly. This will account for the non-energy-conserving character of the hyperfine interaction. Assuming a uniform electron wavefunction $\psi(\mathbf{r})=\sqrt{2 / N \nu_{0}}$ spanning over $N$ nuclei in the QD of volume $\nu_{0} N / 2$, one can first derive the electron-induced relaxation rate $T_{1 e}^{-1}$ of nuclear spins due to the temporal fluctuations of the flip-flop term $\propto\left(\hat{S}_{-}^{e} \hat{I}_{+}+\hat{S}_{+}^{e} \hat{I}_{-}\right) h_{1}(t)$ of the hyperfine coupling (Abragam, 1961, Eble et al. 2006, Meier and Zakharchenya, 1984):

$$
T_{1 e}^{-1}=\left(\frac{\tilde{A}}{N \hbar}\right)^{2} \frac{2 f_{e} \tau_{c}^{e}}{1+\left(\omega^{e} \tau_{c}^{e}\right)^{2}}
$$

where $\hbar \omega^{e}=g_{e} \mu_{\mathrm{B}}\left(B_{z}+B_{n}\right)$ is the electron spin splitting in the total field, $\tau_{c}^{e}$ the correlation time of the hyperfine perturbation $\hat{H}_{1}(t)$, and $f_{e}$ is the fraction of time that the QD contains an unpaired electron. The average constant $\tilde{A}$ of the hyperfine interaction in the QD is used to obtain an expression for $T_{1 e}^{-1}$ independent of the nuclear species. Let us recall that the $A^{j}$ 's vary indeed only slightly for different nuclear spins $I^{j}$ in InAs/GaAs QDs, see table II. In this limit of homogeneous coupling, the approximation of the existence of a high nuclear spin temperature is valid (Abragam, 1961) and allows one to derive a simple differential equation for the dynamic polarization of the average nuclear spin $z$ component per nucleus $\left\langle\tilde{I}_{z}\right\rangle=\frac{1}{N} \sum_{j}\left\langle\hat{I}_{z}^{j}\right\rangle$ in an external magnetic field along $z$ (Dyakonov and Perel., 1974):

$$
\frac{d\left\langle\tilde{I}_{z}\right\rangle}{d t}=-\frac{1}{T_{1 e}}\left[\left\langle\tilde{I}_{z}\right\rangle-\widetilde{Q}\left(\left\langle\hat{S}_{z}^{e}\right\rangle-\left\langle\hat{S}_{z}^{e}\right\rangle_{0}\right)\right]-\frac{\left\langle\tilde{I}_{z}\right\rangle}{T_{d}}
$$

The first term in the right-hand side of Eq. 19 is the DNP source driven by the departure of the electron spin from its thermal equilibrium $\left(\left\langle\hat{S}_{z}^{e}\right\rangle-\left\langle\hat{S}_{z}^{e}\right\rangle_{0}\right)$. It is strictly zero when the electron-nuclear spin system is in thermal equilibrium. Note that the equilibrium value of nuclear spin $\left\langle I_{z}\right\rangle_{0}$ is assumed to be zero in the usual experimental

\footnotetext{
5 The validity of the spin temperature concept in InAs/GaAs QDs in the presence of nuclear quadrupole effects is investigated in detail in section VI.F
} 
conditions $^{6}$. The factor $\widetilde{Q}=\sum_{j} I_{j}\left(I_{j}+1\right) /(N S(S+$ 1)) is a numerical constant which amounts to $\sim 15$ for realistic $\mathrm{In}_{1-x} \mathrm{Ga}_{x}$ As QDs containing a fraction $x \sim 0.5$ of Gallium.

The second term in Eq. (19) accounts for the return to equilibrium of the average nuclear spin following an exponential decay with the time constant $T_{d}$. This term is necessary because the stationary solution of Eq. (19) without nuclear spin relaxation would lead to nuclear polarization much higher than what has been observed experimentally. Different mechanisms may contribute to this relaxation: (i) the dipolar interaction between nuclei responsible for fast depolarization in a very weak field and for a slower field-independent spin diffusion. (ii) Because of local anisotropic strain, the quadrupolar coupling with local electric field gradients introduced in Sec. III.B could also produce an important reduction of the nuclear polarization $z$ component in magnetic fields, depending on the exact angle $\theta$ defined in Fig.7(b) of the principal axis given by the electric field gradients with respect to the quantization axis $z$ Huang and $\mathrm{Hu}$, 2010). This effect will be discussed in more detail in the next Sec.V.B.2. For InAs QDs, the experimental evidence suggests that the quadrupolar fields not only render point (ii) important in many experiments, but also suppress the contribution of argument (i) to nuclear spin relaxation. It is worth noting that in a non-zero magnetic field, say above a few $10 \mathrm{mT}$, the nuclear polarization can survive for minutes or even hours as long as the QD is neither excited (i.e. does not contain any charge carrier) nor coupled to a Fermi sea (Chekhovich et al. $2010 \mathrm{~b}$, Maletinsky et al. 2009). The decay of nuclear polarization must therefore contain a term associated to the presence of the electron in the QD and proportional to $f_{e}$. The associated relaxation mechanism originates most likely from the temporal fluctuations of the Knight field $h_{1}(t)(\tilde{A} / N)\left(\hat{S}_{z}^{e}(t)-\left\langle\hat{S}_{z}^{e}\right\rangle\right) \hat{I}_{z}$ coupled to quasi-static nondiagonal perturbations experienced by the nuclei like the dipole-dipole (Gammon et al., 2001) or quadrupolar interactions (Huang and $\mathrm{Hu}, 2010)$ as detailed in the next Sec.V.B.2. The time-dependent quadrupolar interaction associated to the fluctuations of electrical gradients induced by the creation or annihilation of an exciton could also directly contribute to the relaxation as estimated for donors in GaAs (Paget et al., 2008).

Because of the dependence of $T_{1 e}^{-1}$ on the generated nuclear field $B_{n, z} \equiv 2 \tilde{A} \sum_{j}\left\langle\hat{I}_{z}^{j}\right\rangle /\left(g_{e} \mu_{\mathrm{B}}\right)$, Eq. [19] acquires a non-linear character. Its steady state solution, that would be equivalent to Eq. (17) in the case of a constant $T_{1 e}^{-1}$ and $T_{d} \propto B_{z}^{2}$, gives rise to an implicit equation for

\footnotetext{
${ }^{6}\left\langle I_{z}\right\rangle_{0}$ on the order of $10^{-5}$ to $10^{-4}$ at several Tesla is negligible compared to $\left\langle I_{z}\right\rangle$ on the order of up to about $50 \%$ achieved via optical pumping (Urbaszek et al. 2007).
}

$B_{n, z}$ reading (Eble et al. 2006):

$$
B_{n, z}=\frac{2 \tilde{A} \widetilde{Q}}{g_{e} \mu_{\mathrm{B}}} \frac{\left\langle\hat{S}_{z}^{e}\right\rangle-\left\langle\hat{S}_{z}^{e}\right\rangle_{0}}{1+\varsigma\left[1+\left(g_{e} \mu_{\mathrm{B}}\left(B_{z}+B_{n, z}\right) \tau_{c}^{e} / \hbar\right)^{2}\right]}
$$

where $\varsigma=\left(\frac{N \hbar}{\tilde{A}}\right)^{2} /\left(2 \tau_{c}^{e} f_{e} T_{d}\right)$ is a constant corresponding to the ratio $T_{1 e} / T_{d}$ when the total magnetic field vanishes (i.e. $\left.\left|B_{z}+B_{n, z}\right| \ll \hbar /\left(g_{e} \mu_{\mathrm{B}} \tau_{c}^{e}\right)\right)$. It determines the minimal value $(1+\varsigma)^{-1}$ of the leakage factor limiting the magnitude of the nuclear polarization. Equation 20 shows that the effect of $\varsigma$ is amplified by $\left[1+\left(g_{e} \mu_{\mathrm{B}}\left(B_{z}+B_{n, z}\right) \tau_{c}^{e} / \hbar\right)^{2}\right]$ which represents the influence of the electron spin splitting on the flip-flop rate. Clearly, this introduces a dependence on the relative signs of $B_{n, z}$ and $B_{z}$. The build-up of $B_{n, z}$ is favored when both fields point in opposite directions (i.e. partially compensate each other), whereas DNP tends to be inhibited when the fields are parallel, as confirmed experimentally (Eble et al. 2006). Moreover this simple formalism predicts possible regimes of nuclear field bistability since Eq. 20 is actually a $3^{r d}$ order polynomial equation that may accept three real solutions (2 stable, 1 unstable) determined by its coefficients i.e. the experimental conditions (Braun et al. 2006b, Maletinsky et al. 2007b).

\section{Dynamic nuclear polarization in the presence of nuclear quadrupole effects}

A quantitative description of DNP achievable in optical pumping experiments in QDs has to take into account the nuclear quadrupole interaction introduced in Sec.III.B induced by strain and alloy disorder (Bulutay, 2012). In addition to zero order effects, the quadrupolar interaction is also responsible for a specific mechanism of nuclear spin relaxation which arises even for small $\theta$ and which is induced by the longitudinal part $\propto \hat{S}_{z}^{e} \hat{I}_{z}$ of the hyperfine interaction fluctuations. If the quadrupolar axis is tilted by an angle $\theta$ the momentum operator $\hat{I}_{z}$ couples the nuclear eigenstates of angular momentum difference $\left|\Delta m_{z^{\prime}}\right|=1$ (except the pair of levels $m_{z^{\prime}}= \pm 1 / 2$ ) which tends to equalize the populations of these states and therefore cancel their respective polarization. In the interaction representation and for small $\theta$, the time-dependent coupling is proportional to $\left(h_{1}(t) A / N\right) \theta \omega_{\mathrm{Q}} / \Delta \omega_{n}$ where the relevant energy detuning is the sum of external field and contributions from the nuclear quadrupole effects $\Delta \omega_{n}=\left(\gamma_{n} B_{z}+n \omega_{\mathrm{Q}}\right)$ where $n=m_{z^{\prime}}+1 / 2$ corresponds to the energy splitting between the states $\left|m_{z^{\prime}}\right\rangle$ and $\left|m_{z^{\prime}}+1\right\rangle$. Remarkably, in contrast to the flip-flop relaxation induced by the transverse part of the hyperfine interaction, no electron spin-flip is required if we take into account the non-collinear hyperfine coupling $\hat{H}_{\mathrm{hf}}^{\mathrm{nc}}$ given by Eq. 15 . This makes the associated relaxation independent of the electron spin splitting. Besides, since the nuclear splitting $\Delta \omega_{n} \sim 10 \mathrm{neV}$ 

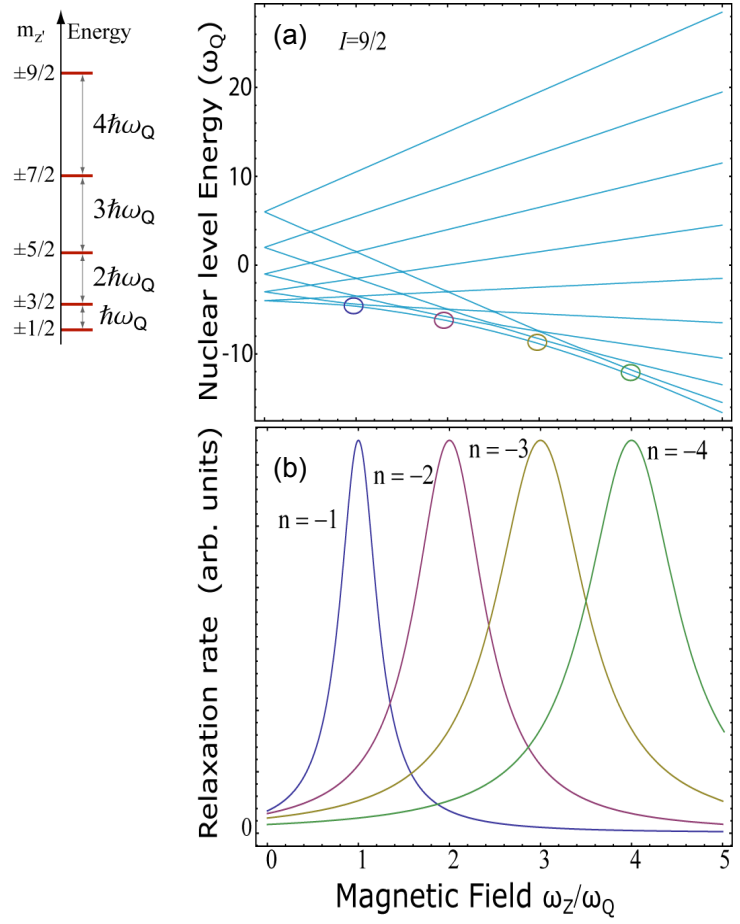

FIG. 19 (a), (b) Energy levels and relaxation rates according to Eq.(21) for a spin $I=9 / 2$ and $\theta=0.05$. The anti-crossings are marked by circles of different color in (a), the corresponding relaxation rates are shown in (b) in the same color.

is small in usual experimental conditions compared to the inverse correlation time $\left(\tau_{c}^{e}\right)^{-1} \sim 10 \mu \mathrm{eV}$ determined in Sec.V.B.3, the issue of energy conservation does not require special consideration and the spectral density of $h_{1}(t)$ reads simply $2 \tau_{c}^{e} /\left(1+\left(\Delta \omega_{n} \tau_{c}^{e}\right)^{2}\right) \approx 2 \tau_{c}^{e}$. Because of the non-harmonicity produced by the quadrupolar splitting $\left(\Delta \omega_{n}\right.$ depends on $\left.n\right)$ it is not possible to derive a single relaxation time for the nuclear polarization, as done for the flip-flop term in Sec.V.B.3. However, the transition rate between the specific levels $|n \pm 1 / 2\rangle$ where $n \in\{-I+1 / 2, \ldots, I-1 / 2\}$ can still be calculated perturbatively according to Redfield's theory (Abragam, 1961. Slichter, 1990) or from the Fermi's golden rule assuming a Lorentzian broadening $\hbar / \tau_{c}^{e}$ of the level spectral density (Huang and $\mathrm{Hu}, 2010$ ). To first order in $\theta$ we obtain
$7:$

$$
\frac{1}{T_{\mathrm{e}-\mathrm{Q}}^{I, n}}=\left(\frac{A_{I}}{2 \hbar N}\right)^{2} \frac{f_{e} \tau_{c}^{e}}{1+\left(\Delta \omega_{n} / c_{I, n} \theta \omega_{\mathrm{Q}}\right)^{2}}
$$

where $c_{I, n}=n \sqrt{(I+1 / 2)^{2}-n^{2}}$. For each pair of levels the relaxation rate is maximum at the magnetic field $B_{z}=-n B_{\mathrm{Q}}$ corresponding to a minimum of $\Delta \omega_{n}$ and follows a Lorentzian dependence with a typical width given by $2 c_{I, n} \theta \omega_{\mathrm{Q}}$. Figure 19 (b) shows the relative evolution of the different rates for a spin $I=9 / 2$ (case of Indium) and assuming a small angle $\theta=0.05$. This magnetic field dependent nuclear spin relaxation mechanism will be considered in the following section that details the evolution of DNP in measurements at variable magnetic fields.

\section{Nuclear field bistability in magnetic field sweeps}

For a given set of experimental parameters there exist several stable nuclear spin configurations, see Eq.20. The experimentally achieved nuclear spin polarization degree will in addition depend on the history of the experiment (non-Markovian behaviour). Different bistability regimes of DNP were observed in single QD optics by varying continuously different parameters in the experiment like the polarization of the optical excitation (Braun et al. 2006b), the laser excitation power (Belhadj et al., 2008; Kaji et al., 2008; Skiba-Szymanska et al., 2008: Tartakovskii et al., 2007) or more commonly the external magnetic field (Braun et al., 2006b; Kaji et al. 2008: Krebs et al., 2008 Maletinsky et al., 2007b). Figure 20(a) shows the typical evolution of the PL from an $X^{+}$trion as a function of a the applied longitudinal magnetic field when the QD is excited with a quasi resonant, $\sigma^{-}$polarized laser. The Zeeman splitting measured between the $\sigma^{+}$and $\sigma^{-}$components increases steadily up to a critical field $B_{c} \approx 4.5 \mathrm{~T}$ where it undergoes an abrupt increase. This provides direct evidence that up to $B_{c}$, a nuclear field $B_{n}$ is created in the quantum dot in the direction opposite to $B_{z}$. When the magnetic field is swept back, a similar abrupt reduction of the Zeeman splitting takes place but at a lower critical field $B_{c}^{\prime} \approx 1 \mathrm{~T}$.

The Overhauser shift $\hbar \omega_{O S}^{e} \equiv\left|g_{e} \mu_{\mathrm{B}} B_{n, z}\right|$ due to the nuclear field can be extracted as shown in Fig.20(b) by subtracting the Zeeman splitting of the trion $\left(g_{h}-\right.$ $\left.g_{e}\right) \mu_{\mathrm{B}} B_{z}$ exclusively due to the external field, where $g_{h} \simeq 2.4$ is the $g$-factor of the heavy-hole pseudo-spin $1 / 2$ for this particular $\mathrm{QD}$ and $g_{e} \simeq-0.6$. Note that the Zeeman splitting used for reference is precisely determined from a measurement where $B_{n}=0$ : under linearly

\footnotetext{
7 Please note that as a consequence of keeping only first order terms in $\theta$ for small angles we can neglect transitions with $\Delta I_{z}=$ 2 .
} 

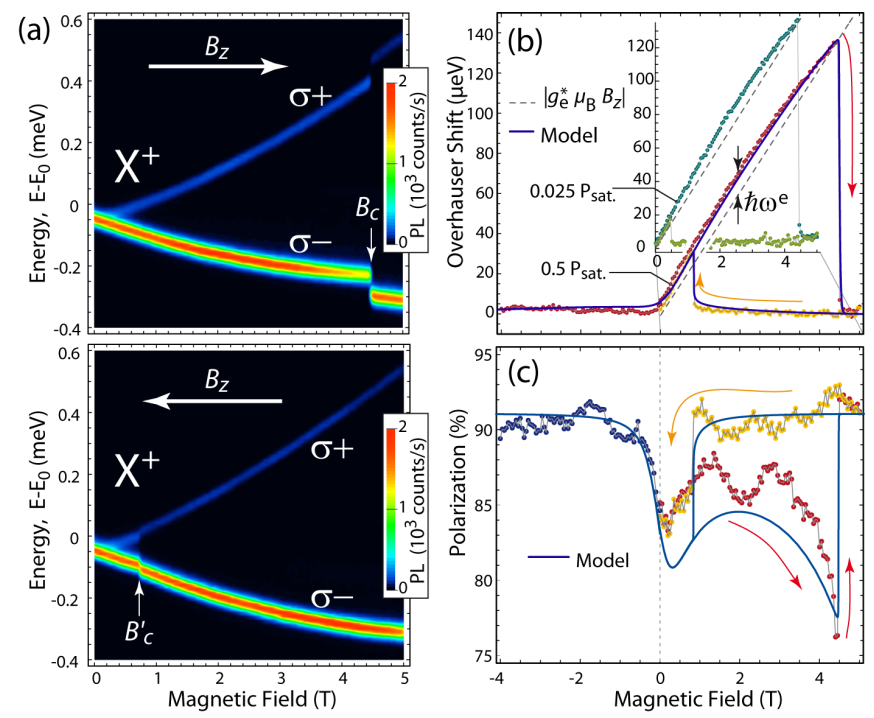

FIG. 20 (a) Density plots of $X^{+}$PL intensity from a single $\mathrm{QD}$ at $\mathrm{T}=1.7 \mathrm{~K}$ as a function of an increasing or decreasing magnetic field $B_{z}$, detected over a $1 \mathrm{meV}$ energy range around $E_{0}=1.3143 \mathrm{eV}$. The quasi-resonant $\sigma^{-}$polarized excitation is $\sim 60 \mathrm{meV}$ above the trion line in zero field. (b) Absolute Overhauser shift of the Zeeman splitting for two excitation powers, and (c) circular polarization as a function of $B_{z}$. The measurement at $0.025 P_{\text {sat }}$ has been shifted and slightly reduced in size for clarity. adapted from Krebs et al. (2008)

polarized excitation in moderate external fields (below $\sim 3 \mathrm{~T}$ ) since in this case $\left\langle\hat{S}_{z}^{e}\right\rangle=0$ due to optical pumping, $\left\langle\hat{S}_{z}^{e}\right\rangle_{0} \approx 0$ due to thermalisation result in zero nuclear polarization. The asymmetrical dependence of $B_{n}$ on $B_{z}$ and the bistability regimes of DNP are clearly evidenced in Fig. 20(b). Remarkably, the Overhauser shift grows almost linearly with the positive $B_{z}$ (for $\sigma^{-}$excitation) in order to remain above the dashed line which represents the electron Zeeman splitting. As long as this condition is fulfilled, the nuclear field $B_{n}$ fully compensates the applied field (even slightly overcompensates) so that the amplification of $\varsigma$ in Eq. 200 remains moderate of the order of unity. When $B_{z}$ approaches the critical field $B_{c}$, the electron spin splitting $\hbar \omega^{e}$ vanishes, indicating that the nuclear field has reached its maximum in the conditions of the experiment. Beyond this point a further increase of $B_{z}$ yields a reduction of $B_{n, z}$. This provides a negative feedback ( $\varsigma$ in Eq. 200 is amplified) and the Overhauser shift suddenly drops as the applied field is slightly increased. The maximum Overhauser shift of $137 \mu \mathrm{eV}$ achieved here would amount to an average nuclear polarization of $43 \%$ for a pure InAs QD, but it is certainly closer to $60 \%$ for a more realistic $\mathrm{In}_{0.5} \mathrm{Ga}_{0.5} \mathrm{As}$ intermixed QD, using the values for $100 \%$ nuclear polarization in table III as references. By adjusting the parameters of the model it is possible to reproduce the experimental magnetic field dependence of $\hbar \omega_{O S}^{e}$ reasonably well using the numerical solution of Eq. (19). In practice only $\tau_{c}^{e}, g_{e}$ and the product $f_{e} T_{d}$ have to be var- ied for the fit, whereas other parameters can be fixed to the values expected for usual InAs QDs $\left(N=5 \cdot 10^{4}\right.$, $\tilde{Q}=13, T=1.7 \mathrm{~K})$.

To improve the agreement around zero field where the experimental slope $\left(\partial \omega_{O S}^{e} / \partial B_{z}\right)$ presents a kind of discontinuity, it is necessary to include a field-dependent nuclear relaxation rate $T_{d}^{-1}$. One plausible approach is to include the gradual inhibition of nuclear spin relaxation due to the quadrupolar coupling as the longitudinal magnetic field increases (Abragam, 1961; Huang and $\mathrm{Hu}$, 2010, Meier and Zakharchenya, 1984). In realistic QDs we have to average over the distribution of quadrupolar interactions both in magnitude and direction, and hence the relaxation resonances in Eq. (21) are drastically broadened compared to the estimations shown in Fig.19. Also, the applied longitudinal field will restore the eigenaxis along $z$. This justifies the incorporation of a simplified magnetic field dependence as we assume in addition to a constant term a Lorentzian part describing qualitatively the slowdown of relaxation at high fields:

$$
T_{d}^{-1}=T_{d_{\infty}}^{-1}+\frac{T_{d_{0}}^{-1}}{1+\left(B_{z} / B_{Q^{\prime}}\right)^{2}}
$$

A manual fit of the model to the experimental points is plotted in Fig. 20.(b) (solid line) with $\tau_{c}^{e}=75 \mathrm{ps}, g_{e}=$ -0.6 for the electron g-factor ${ }^{8}, f_{e} T_{d_{0}}=65 \mu \mathrm{s}, f_{e} T_{d_{\infty}}=$ $5 \mathrm{~ms}$ and $B_{Q^{\prime}}=0.4 \mathrm{~T}$, where $B_{Q^{\prime}}$ is a measure of the strength of the nuclear quadrupole effects, typically on the order of $100 \mathrm{mT}$. The inclusion of this magnetic field dependent nuclear spin relaxation rate improves the fit considerably for $B_{z}<1 \mathrm{~T}$ (Krebs et al. 2008$)$.

In the present simple model the fraction of time of QD occupation $f_{e}$ and the relaxation time $T_{d}$ can not be distinguished since only their product appears in $\varsigma$. Furthermore, when the laser excitation power (proportional to $f_{e}$ below the saturation power $P_{\text {sat }}$ ) is reduced from $P_{\text {sat }} / 2$ to $P_{\text {sat }} / 40$, the generation of the nuclear field is almost not affected as shown in Fig. 20(b): despite a smaller initial value in zero field, the Overhauser shift still follows the increase of $B_{z}$ up to about the same critical field $B_{c}$ around $4.5 \mathrm{~T}$, reaching the same maximum Overhauser shift $\simeq 135 \mu \mathrm{eV}$ with $P_{\text {sat }} / 40$ as achieved with $P_{\text {sat }} / 2$. This is a clear indication that while the power decreases the relaxation time $T_{d}$ increases. To reach the same $B_{c}$ in the simulations an almost constant product $f_{e} T_{d}$ is required. It can therefore be inferred that $T_{d} \propto f_{e}^{-1}$ in qualitative agreement with the very

\footnotetext{
${ }^{8}$ It was assumed that the hyperfine interaction with the hole reduces the theoretical Overhauser shift $\left|2 \tilde{A}\left\langle I_{z}\right\rangle\right|$ by about $10 \%$, see Sec.VII.B This enables us to use the nominal value expected for the electron $g$-factor instead of the reduced effective factor $g_{e}^{\star}=0.9 g_{e}$ determined from the maximum Overhauser shift $\hbar \omega_{O S}^{e}$ at $B_{c}$ and corresponding to the dashed line in Fig. 20. b). This is not a critical assumption since it improves only slightly the agreement with the experimental data in weak fields.
} 
slow nuclear relaxation observed when the sample is left in the dark i.e. does not contain any charge carriers (see Sec.VI.F . This observation points towards a depolarization mechanism due to Knight field fluctuations coupled to the quasi-static local dipolar field (Gammon et al. 2001) or to the quadrupolar interaction (Huang and $\mathrm{Hu}$, 2010) as the dominant cause of nuclear spin relaxation in optically excited QDs.

The $X^{+}$is created through quasi-resonant optical excitation. The electron-nuclear spin flip-flop can either occur during the trion lifetime $\tau_{r} \sim 1$ ns, or alternatively, during the shorter stage of energy relaxation from the initial excited level since the correlation time extracted from the fits $\tau_{c}^{e} \ll \tau_{r}$. A reduction of $\left\langle S_{z}^{e}\right\rangle=-\rho_{c} / 2$ due to this spin flip-flop is expected and we shall consider if this decrease is observable in realistic experiments: From Eq. (19) one can infer the spin-flip time $T_{1 n}$ experienced by the electron due to the nuclei to be $T_{1 n}=$ $T_{1 e} f_{e} /(N \tilde{Q})$. From the fitting parameters used above, it typically amounts to $\sim 10 \mathrm{~ns}$ in the magnetic field range where a non-zero Overhauser shift is created. The probability of an electron spin-flip during its lifetime is therefore not completely negligible $\left(\tau_{r} / T_{1 n} \approx 0.1\right)$ and the polarization of the photons emitted by $X^{+}$recombination should be affected by the preceding electron-nuclear spin flip-flop process. Also, when the electron splitting $\hbar \omega^{e}$ becomes of the order of $\tilde{A}(\tilde{Q} / N)^{1 / 2} \sim 1 \mu \mathrm{eV}^{9}$, the first order effect of spin precession in the nuclear field fluctuations (as discussed in Sec.IV.A) becomes predominant and should appreciably enhance the electron spin relaxation because $T_{\Delta} / T_{1 n} \sim \tilde{A} \tau_{c}^{e} / \hbar(\tilde{Q} / N)^{1 / 2} \sim 0.1$ (Krebs et al. 2008). This is confirmed experimentally as a clear correlation between the measured $X^{+}$polarization and the achieved Overhauser shift is found, see Fig. 20(c). The polarization is reduced by $\sim 5 \%$ in the conditions where DNP is built up efficiently i.e. when $T_{1 n}$ is short. More specifically the $X^{+}$polarization exhibits a pronounced dip down to $76 \%$ when approaching the critical field $B_{c}$, immediately followed by an abrupt increase to a roughly constant level of $91 \%$. For a qualitative comparison the theoretical reduction of the polarization $\rho_{c}$ of the $X^{+}$emission is plotted in Fig.20(c) using $\rho_{c}=\rho_{c}^{\max } /\left(1+\tau_{r} / T_{1 n}\right)$ with $\rho_{c}^{\max }=0.91$ and $\tau_{r}=0.7 \mathrm{~ns}$. This formula is only approximate because it assumes that electron-nuclei flip-flops take place for the whole radiative lifetime of $X^{+}$although the correlation time used in the model is much shorter, but on the other hand it neglects the faster spin relaxation rate $\sim T_{\Delta}^{-1}$ near $B_{c}$. The general idea is captured: the smaller the splitting in energy $\left|\hbar \omega^{e}\right|$ between electron spin up and spin down states, the shorter the spin lifetime and consequently the smaller the measured polarization, irrespective of the actual spin-flip mechanism. The dip observed at $B_{c}$ is clearly related to

\footnotetext{
9 The secular approximation used to derive the expression of $T_{1 n}$
} to the second order in perturbation is no longer valid.
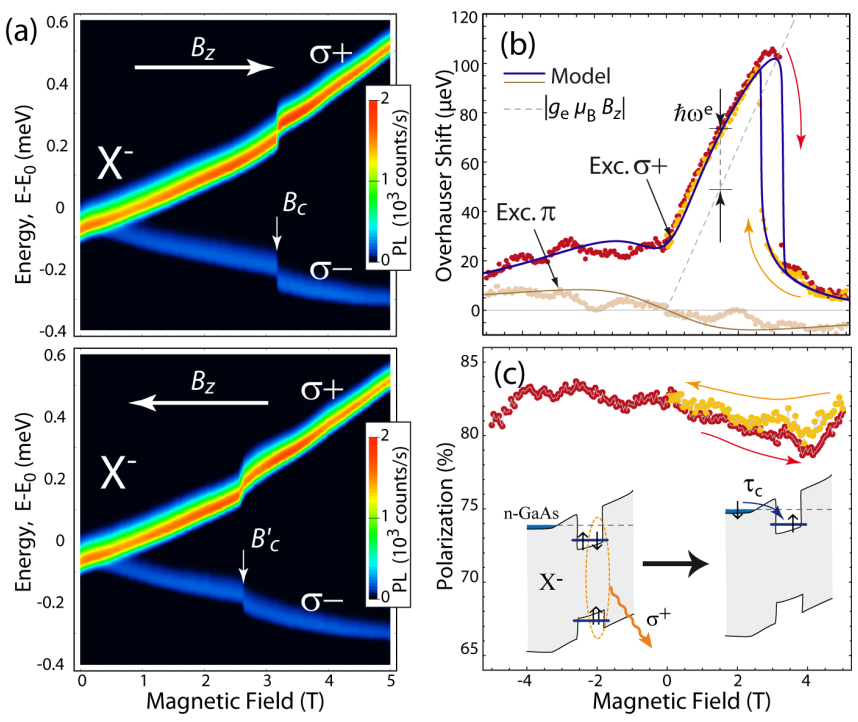

FIG. 21 (a) Density plots of $X^{-}$PL intensity from the same QD of Fig. 20 at $\mathrm{T}=1.7 \mathrm{~K}$ as a function of an increasing or decreasing magnetic field $B_{z}$, detected around $E_{0}=1.305 \mathrm{eV}$. The quasi-resonant $\sigma^{+}$or $\pi$ polarized excitation is $\sim 40 \mathrm{meV}$ above the trion line in zero field. (b) Overhauser shifts of the Zeeman splitting for both excitation polarizations with superimposed fits of the model using the same parameters (solid lines). The measurements under $\pi$ polarization, performed in positive fields, have been duplicated anti-symmetrically in negative fields. (c) Circular polarization under $\sigma^{+}$excitation as a function of $B_{z}$ showing no correlation to the Overhauser shift in contrast to $X^{+}$. Inset illustrates schematically $X^{-}$ emission, followed by the capture of a second resident electron defining the correlation time $\tau_{c}^{e} \sim 20 \mathrm{ps}$ in the model. adapted from Krebs et al. (2008)

the vanishing $\left|\hbar \omega^{e}\right|$. It is remarkable that the Zeeman splitting of the electron is zero in an applied field $B_{z}$ of more than 4 Tesla, and an electron depolarization due to the Merkulov effect is observed, in analogy to the observed decrease in electron polarization around zero magnetic field (Braun et al., 2005; Merkulov et al., 2002).

Figure 21] shows for comparison similar measurements for the same QD but in the negative charge state giving rise to the PL emission of an $X^{-}$line located about $10 \mathrm{meV}$ below the $X^{+}$line. This charge state is achieved by increasing the gate voltage applied to the $n$-Schottky device by $+0.8 \mathrm{~V}$, in a regime where the QD is actually charged by two electrons. The quasi-resonant optical excitation creates a hole confined in the QD valence ground state and an electron in an excited state which escapes from the dot in a few picoseconds. This leads to $X^{-}$ formation in a polarization state determined by the hole spin (Laurent et al., 2005). Non-linear DNP is again observed when a longitudinal magnetic field is applied, see Fig. 21(a),(b), with some notable differences compared to the $\bar{X}^{+}$case:

(i) the Overhauser shift develops when the $\sigma^{+}$branch of the trion (i.e. the Zeeman line which blue-shifts with the applied magnetic field) is predominantly pumped by the 
$\sigma^{+}$excitation. This inversion of the required polarization with respect to $X^{+}$case results from the symmetry between $X^{+}$and $X^{-}$(Eble et al. 2006$)$ : As shown in Fig. 21(c) inset, a $\sigma^{+}$polarized $X^{-}$does not itself polarize the nuclei because the conduction electrons are in a singlet state, but after recombination with the $\Uparrow$ hole, it yields an unpaired electron with $\uparrow$ spin exactly as for a $\sigma^{-}$photo-created $X^{+}$trion. The same regime of DNP can therefore take place in a positive magnetic field which is still opposite to the generated nuclear field $B_{n}$.

(ii) In contrast to $X^{+}$, the measured PL polarization in Fig.21(c) shows no correlation with the measured Overhauser shift, because it is determined by the optically created hole spin polarization.

(iii) The effective electronic splitting $\left|\hbar \omega^{e}\right| \simeq 25 \mu \mathrm{eV}$ is significantly larger, the critical magnetic fields $B_{c} \simeq 3.3 \mathrm{~T}$ and $B_{c}^{\prime} \simeq 2.8 \mathrm{~T}$ associated with the abrupt decrease and recovery of nuclear polarization are respectively reduced and enhanced, which results in a narrower domain of bistability, and eventually there is only a partial decrease of $\left|\hbar \omega_{\text {OS }}^{e}\right|$ in negative fields leading to a residual shift of $\sim 15 \mu \mathrm{eV}$ at $B_{z}=-5 \mathrm{~T}$.

All these changes can still be reproduced by the above model as shown by the simulation plotted in Fig. 21.(b) (solid line) assuming $\tau_{c}^{e}=18 \mathrm{ps}, g_{e}=-0.62, f_{e} T_{d_{0}}=$ $1 \mathrm{~ms}, f_{e} T_{d_{\infty}}=8.7 \mathrm{~ms}$ and $B_{Q^{\prime}}=0.7 \mathrm{~T}$. The reduction of the non-linearity quantified by $\left(B_{c}-B_{c}^{\prime}\right) /\left(B_{c}+B_{c}^{\prime}\right)$ results essentially from the shortening of $\tau_{c}^{e}$ which leads to a smoothing of the Lorentzian factor in the right-hand side of Eq. 20 and makes the condition $\hbar \omega^{e} \approx 0$ less critical. This explains also why in negative fields as well as for $B_{z}>B_{c}$, a finite Overhauser shift can still be generated. There is however a clear asymmetry between the measured degree of DNP at $B_{z}=+5 \mathrm{~T}$ and $-5 \mathrm{~T}$ indicating a favored DNP in large negative fields. In this situation the electron splitting $\hbar \omega^{e}$ is dominated by the Zeeman part so that the flip-flop rate $T_{1 e}^{-1}$ should be essentially symmetrical. The reason for the asymmetry comes now from the increase of the equilibrium spin polarization $2\left\langle S_{z}^{e}\right\rangle_{0}$ which amounts to $\pm 53 \%$ at $T=1.7 \mathrm{~K}$ and $B_{z}= \pm 5 \mathrm{~T}$. The departure from equilibrium in Eq. (20) is therefore drastically altered according to the field direction. The role played by this equilibrium spin $\left\langle S_{z}^{e}\right\rangle_{0}$ is confirmed in measurements with a linearly polarized $(\pi)$ excitation which does not directly create any spin polarization $\left(\left\langle S_{z}^{e}\right\rangle=0\right)$, but still show a finite Overhauser shift in high fields, see Fig.21(b).

\section{Nonlinearity of the nuclear polarization as a function of excitation laser polarization}

The case of single $X^{-}$trions is particularly interesting because the circular polarization of the corresponding PL line allows for a direct determination of the unpaired electron spin left in the QD just after the optical recombination and responsible for the subsequent DNP. The validity of Eq. 20 which relates the Overhauser shift $\propto\left\langle\hat{I}_{z}\right\rangle$

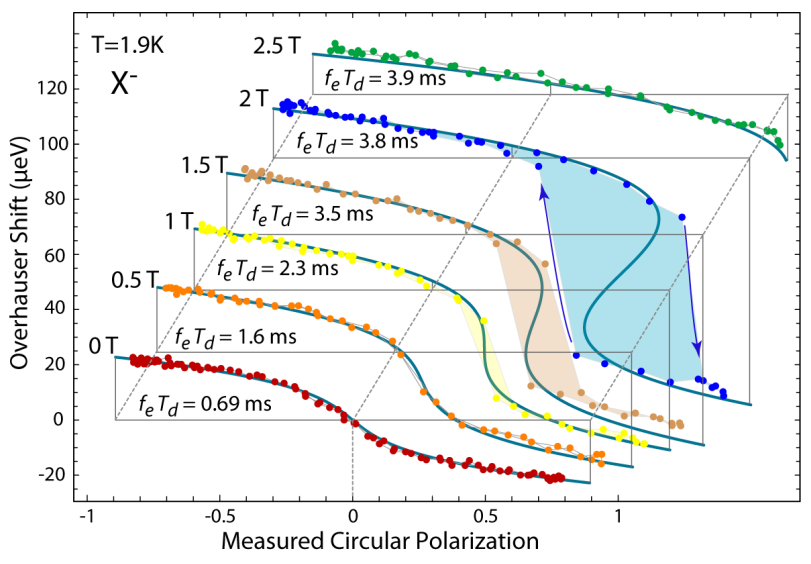

FIG. 22 Overhauser shift $\propto\left\langle\hat{I}_{z}\right\rangle$ in a single InAs/GaAs QD as a function of the measured circular polarization from the $X^{-}$PL line (essentially $\propto\left\langle S_{z}^{e}\right\rangle$ of the electron left behind) around $E_{0}=1.265 \mathrm{eV}$ at different longitudinal magnetic fields. The polarization of the quasi-resonant excitation is varied by stepwise rotating a quarter-wave plate over a total angle of $\pi$, which achieves the complete polarization cycle $\pi \rightarrow \sigma^{+} \rightarrow \pi \rightarrow \sigma^{-} \rightarrow \pi$. Solid lines represent fits of Eq. 20 with $\tau_{c}^{e}=31 \pm 2 \mathrm{ps}, g_{e}^{\star}=0.54$ and the product $f_{e} T_{d}$ adjusted for each field as indicated. The curves and experimental points at different magnetic fields have been translated for clarity.

to the average electron spin $\left\langle S_{z}^{e}\right\rangle$ can thus be tested in a fixed magnetic field without the need of Eq. 22 (Eble et al. 2006). Figure 22 shows a series of such measurements where the polarization of the excitation light is changed stepwise by rotating the quarter-wave plate through which the linearly polarized laser passes, allowing essentially to plot $\left\langle\hat{I}_{z}\right\rangle$ as a function of $\left\langle S_{z}^{e}\right\rangle$. Along a $\pi$ rotation the incident polarization covers the cycle $\pi \rightarrow \sigma^{+} \rightarrow \pi \rightarrow \sigma^{-} \rightarrow \pi$, as a result, the recorded circular polarization degree of the PL varies between -0.8 and +0.8 . For each excitation polarization the $X^{-}$PL emission is measured in $\sigma^{+}$and $\sigma^{-}$polarization separately, typically for a $1 \mathrm{~s}$ integration time which is long enough to reach the steady state regime described by Eq. 20. Fig.22 clearly shows that the DNP achieved via optical pumping depends in a highly non-linear fashion on the average injected electron spin $\left\langle S_{z}^{e}\right\rangle$ for magnetic fields below the critical field $B_{c}$ which amounts to $\simeq 2.2 \mathrm{~T}$ for the $X^{-}$of this QD. This non-linearity manifests itself as an asymmetry of the Overhauser shift as $\left\langle S_{z}^{e}\right\rangle$ changes from positive to negative, in perfect agreement with the asymmetry observed when then magnetic field changes sign, as discussed earlier for Fig. 21.

In zero applied field the Overhauser shift shown in Fig. 22 is as expected exactly anti-symmetrical with respect to a change in sign of $\left\langle S_{z}^{e}\right\rangle$. Interestingly, it still exhibits a non-linear dependence on $\left\langle S_{z}^{e}\right\rangle$ indicating that the nuclear field which develops is strong enough to restrain the DNP rate $T_{1 e}^{-1}$ : a negative feedback develops as any further spin flip-flop will increase $\hbar \omega^{e}=\hbar \omega_{\text {OS }}^{e}$ and hence make the next spin flip-flop less likely than the previ- 


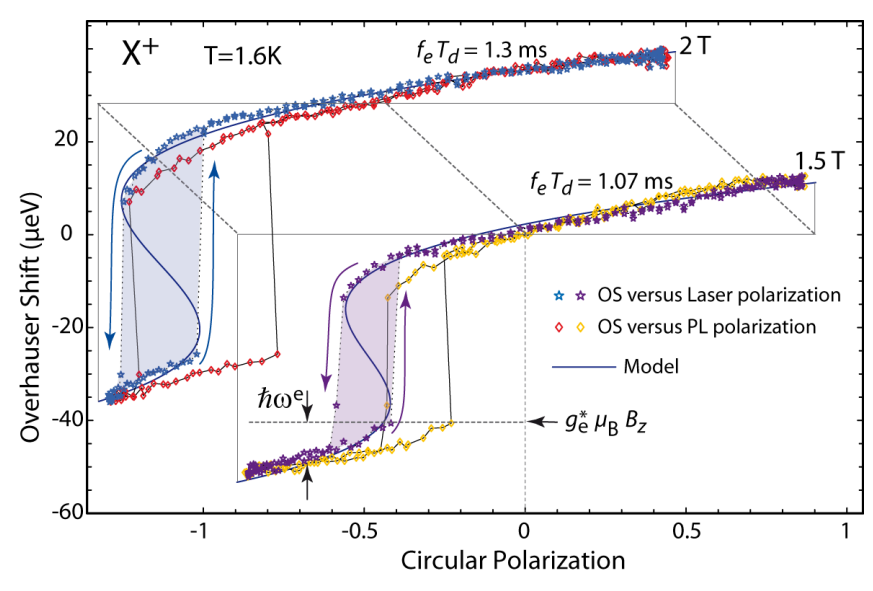

FIG. 23 Overhauser shift in a single InAs/GaAs QD as a function of the Laser or PL circular polarization for an $X^{+} \mathrm{PL}$ line around $E_{0}=1.348 \mathrm{eV}$ at two longitudinal magnetic fields. The polarization of the quasi-resonant excitation is changed by stepwise rotating a quarter-wave plate as in Fig.22. Solid blue lines represent fits of Eq. 20 with $\tau_{c}^{e}=31 \pm 2 \mathrm{ps},\left|g_{e}\right|=$ $0.5,\left|g_{e}^{\star}\right|=0.45$ and the product $f_{e} T_{d}$ as indicated. The curves and experimental points for both magnetic fields have been translated for clarity.

ous one. At higher positive fields, this drastic feedback shifts the non-linear response of the nuclear system towards positive values of $\left\langle S_{z}^{e}\right\rangle$ and enhances its magnitude to the point that a bistability region shows up, as evidenced by the measurements at $2 \mathrm{~T}$ in Fig.22 (Braun et al. 2006b; Maletinsky et al., 2007b). When the external field exceeds $B_{c}$, the level of high nuclear polarization can no longer be reached by increasing the electron spin polarization. The system remains in the low nuclear polarization state which now depends almost linearly on $\left\langle S_{z}^{e}\right\rangle$ because the role of $B_{n, z}$ in $T_{1 e}^{-1}$ is negligibly small $\left(\left|B_{n, z}\right| \ll\left|B_{z}\right|\right)$. Note that there is yet a residual Overhauser shift of a few $\mu \mathrm{eV}$ at $\left\langle S_{z}^{e}\right\rangle=0$ due the finite equilibrium spin $\left\langle S_{z}^{e}\right\rangle_{0}$ in Eq. 20). As shown in Fig.22, the agreement with the model is very good. From Eq. 20p one can express $\left\langle S_{z}^{e}\right\rangle$ as a function of the nuclear field $B_{n, z}$ and therefore determine $\tau_{c}^{e}, f_{e} T_{d}$ and $g_{e}$ from a least-square fit to the experimental points. All the theoretical curves in Fig.22 are obtained with the same set of parameters except for $\tau_{c}^{e}$ and $f_{e} T_{d}$. The estimated values of the correlation time which presumably should not depend on the magnetic field varies indeed very moderately $\left(\tau_{c}^{e}=31 \pm 2 \mathrm{ps}\right)$, while in contrast the product $f_{e} T_{d}$ increases appreciably with the field in qualitative agreement with the Lorentzian dependence assumed in Eq. 222.

Figure 23 shows the analogous evolution of the Overhauser shift for an $X^{+}$trion when the excitation polarization varies from $\sigma^{+}$to $\sigma^{-}$and back. The global behavior is very similar, with a bistability region in magnetic fields below $B_{c}$. The main difference, besides the symmetry change between $X^{+}$and $X^{-}$case already discussed above, is that the Overhauser shift can no longer be satisfactorily correlated to the measured PL circular polarization according to Eq. 20p.

The difference between the laser polarization $\left(\propto\left\langle S_{z}^{e}\right\rangle_{t=0}\right)$ and the $X^{+}$PL polarization $\left(\propto\left\langle S_{z}^{e}\right\rangle_{t=\tau_{r}}\right)$ is a measure of the impact of the electron-nuclei spin flip-flops on the average electron polarization $\left\langle S_{z}^{e}\right\rangle$. As can be expected, the difference between excitation polarization and emitted polarization in Fig.23 is largest when nuclear polarization is strongest, in close analogy to the dip at $B_{c}$ in Fig.20(c). Good agreement with Eq. 20 can still be obtained by assuming $\left\langle S_{z}^{e}\right\rangle$ proportional to the nominal laser polarization, see fits to the data in Fig.23.

Investigating the non-linearity of DNP as a function of the electron or laser circular polarization gives access to the key parameters $\tau_{c}^{e}$ and $f_{e} T_{d}$ without causing their simultaneous variation, as is the case when varying the magnetic field or excitation power. As seen in Fig.22 this can be used to evidence and possibly measure the evolution of the relaxation time $T_{d}$ in magnetic field. In Urbaszek et al. (2007), this method was employed to study the effect of temperature on the magnitude of the optically generated nuclear polarization. Figure 24(a) shows that the Overhauser shift in a magnetic field $B_{z}=2 \mathrm{~T}$ appreciably increases with the temperature in the range $30-55 \mathrm{~K}$, for both $\sigma^{+}$and $\sigma^{-}$excitation polarizations. This general trend could seem rather counterintuitive because both the electron and nuclear spin lifetimes decrease at higher temperatures when they are measured independently ( $\mathrm{Lu}$ et al., 2006 , Sénès et al. 2004). However, the sensitivity of the DNP on the energy cost of electron-nuclei flip-flops can lead to a specific dependence. Indeed, when the temperature is increased one may naturally expect a shortening of the correlation time $\tau_{c}^{e}$. As depicted in Fig. 24(b), the competition between the broadening $\hbar / \tau_{c}^{e}$ of the levels involved in the flip-flop and the energy cost $\hbar \omega^{e}$ reaches a new equilibrium corresponding roughly to $\left|\omega^{e} \tau_{c}^{e}\right| \sim 1$ obtained for a larger nuclear field.

This qualitative interpretation is confirmed by the determination of $\tau_{c}^{e}$ at different temperatures from the fitting of the model to the non-linearity of the Overhauser shift $\delta_{n}=\hbar \omega_{O S}^{e}$ as a function of $\left\langle S_{z}^{e}\right\rangle$, see Fig. 24(c). $\tau_{c}^{e}$ has to be reduced from $60 \mathrm{ps}$ at $5 \mathrm{~K}$ to only $10 \mathrm{ps}$ at $45 \mathrm{~K}$ to neatly reproduce the experimental data, but interestingly this also requires to increase the relaxation time of the nuclei $T_{d}$ (note that in Fig. 24(c), $f_{e}$ is taken as an independent constant). Strikingly, the relative increase of $T_{d}$ is approximately the same as the relative reduction of $\tau_{c}^{e}$. This behavior is expected if the nuclear relaxation is dominated by the Knight field fluctuations produced by the electron in the QD. In this case it can be shown (see Huang and $\mathrm{Hu}(2010))$ that $T_{d}^{-1} \propto f_{e} \tau_{c}^{e} /\left(1+\left(\tilde{\omega}^{n} \tau_{c}^{e}\right)^{2}\right)$ where $\tilde{\omega}^{n}$, the typical nuclear spin-splitting due to external field and quadrupolar interaction, is a small correction that can be neglected since $\left|\tilde{\omega}^{n} \tau_{c}^{e}\right| \ll 1$ for $\tau_{c}^{e} \sim 50$ ps. 
(a)

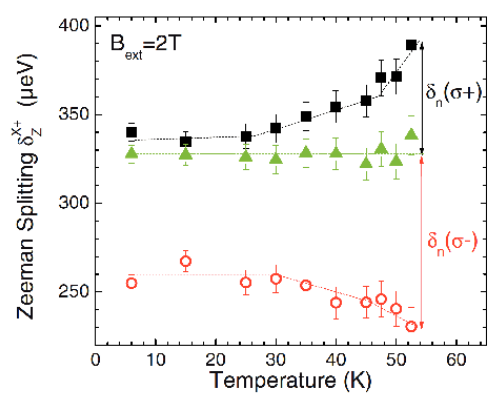

(b)

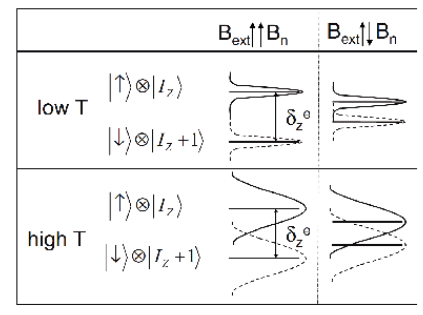

(c)

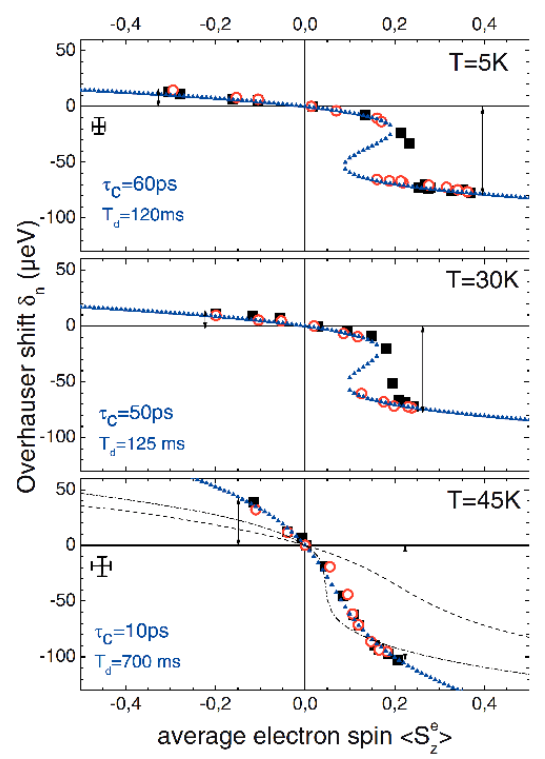

FIG. 24 (a) Spin splitting in a single InAs/GaAs QD for an $X^{+}$PL line as a function of temperature. The black (red) symbols are measured following $\sigma^{+}\left(\sigma^{-}\right)$polarized laser excitation and the green symbols following linear laser excitation, for which DNP is absent. (b) Schematics of the electronnuclei level splitting and the change of broadening due to temperature raising. (c) Evolution of the Overhauser shift as a function of $\left\langle S_{z}^{e}\right\rangle$ deduced from the PL circular polarization $\left(\left\langle S_{z}^{e}\right\rangle=-\rho_{c} / 2\right)$ for different temperatures. Solid lines are fit of the model to the experimental data with the corresponding correlation time $\tau_{c}^{e}$ and relaxation time $T_{d}$ as indicated (after Urbaszek et al. (2007)).

As a result, the effect of the temperature in the range $2-55 \mathrm{~K}$ is essentially governed by the reduction of the correlation time $\tau_{c}^{e}$. On the one hand it produces an increase of both relaxation times $T_{1 e}$ and $T_{d}$, equivalent to the phenomenon of motional narrowing. However, these changes compensate each other and therefore do not affect the magnitude of nuclear polarization. Note that the parameter $\varsigma$ in Eq. 20 is indeed a constant independent of $\tau_{c}^{e}$ when $T_{d}^{-1} \propto \tau_{c}^{e}$. On the other hand, the flip-flop probability is directly favored by the level broadening, which finally gives rise to higher DNP. The reduction of $\left\langle S_{z}^{e}\right\rangle$ with the temperature due to concurrent relaxation mechanisms (Sénès et al. 2004) still limits the effective DNP magnitude that can be reached in this way. 
5. Overhauser effect in zero magnetic field : role of the Knight field

A very remarkable observation in InAs QDs is the occurrence of substantial nuclear spin polarization in zero external magnetic field (Lai et al., 2006). In contrast, in n-type bulk semiconductor samples this requires a small external field of a few millitesla to suppress the nuclear spin relaxation induced by the dipolar interaction (Meier and Zakharchenya, 1984). For each nucleus this spinspin interaction indeed amounts to a randomly oriented local effective magnetic field $B_{\mathrm{L}} \sim 0.15 \mathrm{mT}$ with an associated spin precession period of $T_{2} \sim 100 \mu$ s determining the timescale of both the nuclear spin relaxation and the reorientation of $B_{\mathrm{L}}$. In comparison, the intrinsic nuclear spin decay time $T_{d}$ is in the $1-100 \mathrm{~ms}$ range, from an analysis of the non-linearity (assuming e.g. $f_{e}=0.1$ ) or from direct measurements (see below Sec. VI). The fact that $T_{d} \gg T_{2}$, is definitely a remarkable feature of InAs QDs. In Lai et al. (2006) it was proposed that the electron spin $\left\langle S_{z}^{e}\right\rangle$ could produce a Knight field $\boldsymbol{B}_{\mathrm{K}} \propto\left\langle\boldsymbol{S}^{e}\right\rangle$ (see Eq. (5) in Sec.III.A), strong enough compared to $B_{\mathrm{L}}$ to stabilize the nuclear polarization along $z$ in zero external field. As shown in Fig.25(a) for a QD charged by a single electron, the Overhauser shift is almost constant over the $\pm 6 \mathrm{mT}$ range around zero field, with still a reduction at $\sim \pm 0.6 \mathrm{mT}$ for $\sigma^{\mp}$ circular polarization of excitation. These dips would result from the compensation of the Knight field by the external field, which in turn yields a faster decay of nuclear spin polarization and a reduction of Overhauser shift. They are also observed on the circular polarization of $X^{-}$PL, see Fig.25(b), although in this case the polarization should solely reflect the hole spin polarization. This can be interpreted as (i) the partial inhibition by the nuclear field of the hole spin relaxation due to anisotropic electron-hole exchange during $X^{-}$formation or alternatively (ii) the hole spin itself becoming more sensitive to the nuclear field fluctuations, as discussed in section VII.A. To discuss the Knight field hypothesis more quantitatively, one can use Eq. (17) where the field $\boldsymbol{B}$ experienced by the nuclei is the sum $\boldsymbol{B}=\boldsymbol{B}_{\text {ext }}+\boldsymbol{B}_{\mathrm{K}}$, since the non-linearity due to the field-dependence of Eq. (18) is negligible in this very narrow field range. Accordingly, the Overhauser shift should indeed be reduced at $\boldsymbol{B}_{\text {ext }}=-\boldsymbol{B}_{\mathrm{K}}$. Furthermore, the non-uniform nature of the Knight field allows to explain very satisfactorily both the partial reduction of the Overhauser shift, whereas Eq. (17) predicts complete cancellation, and the enhancement of the corresponding magnetic width compared to $B_{\mathrm{L}}$.

However, the exact role played by the Knight field in stabilizing the nuclear field needs to be clarified in the light of the recently discovered importance of the nuclear quadrupole interaction introduced in Sec.III.B, revealed for instance in experiments carried out in transverse magnetic fields (Krebs et al. 2010): they show that an external field perpendicular to $z$ in the $10-100 \mathrm{mT}$ range, i.e. a few orders of magnitude larger than $B_{\mathrm{L}}$ and $B_{\mathrm{K}}$, does

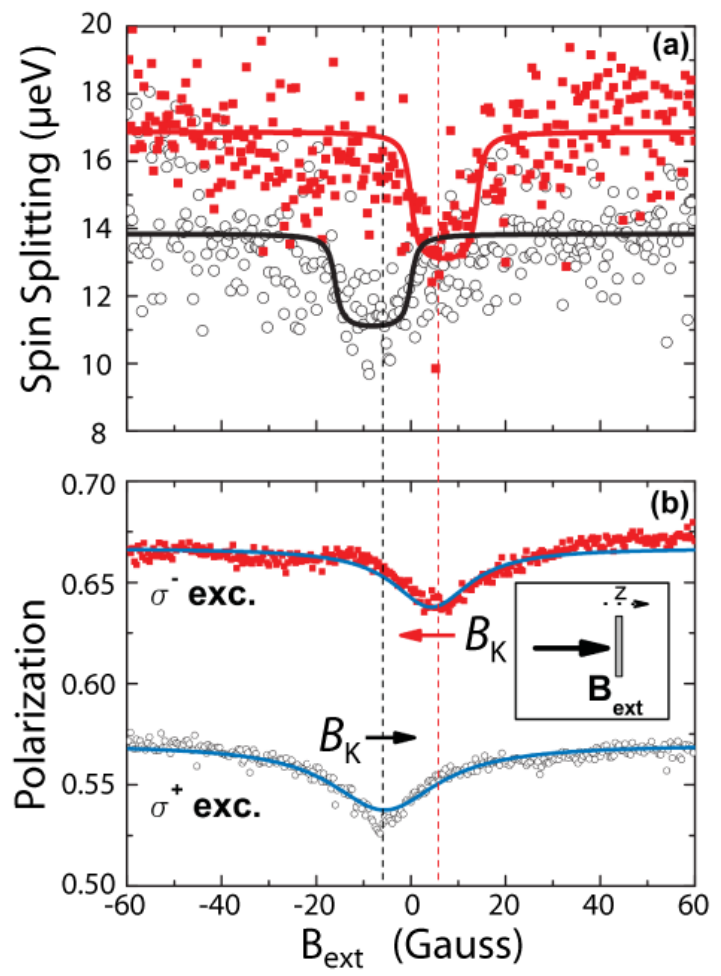

FIG. 25 (a) Overhauser shift and (b) circular polarization of a single $X^{-}$PL line from an InAs/GaAs QD as a function of a small longitudinal magnetic field for $\sigma^{+}$and $\sigma^{-}$circularly polarized excitation. (after Lai et al. $(2006)$ ).

not destroy the nuclear polarization along $z$. In Dzhioev and Korenev (2007) it was suggested that the nuclear quadrupole interaction is more likely to be responsible for allowing DNP at (near) zero applied magnetic field, as depolarization of the nuclei via the dipole-dipole interaction is quenched. This alternative explanation will be reviewed in more detail in Sec.VI.D together with the striking observation of the anomalous Hanle effect. Still, the assignment of the dips observed in Fig.25 to the Knight field compensation is the most likely explanation. Since the polarization of the $\pm 1 / 2$ nuclear spin sublevels is not protected from $B_{\mathrm{L}}$ by the quadrupolar interaction, their sensitivity to any magnetic field of the order of $B_{\mathrm{L}}$ should still reflect the contribution of the Knight field to the stabilization of the total nuclear field.

\section{Nuclear field versus electron-hole exchange : the case of neutral excitons}

The nuclear field generated in QDs under quasiresonant excitation with circularly polarized light is definitely a local property: on the one hand, distinct dots illuminated under the same laser spot of about $1 \mu \mathrm{m}$ diameter exhibit distinctive nuclear field effects; on the other hand all excitonic states in a given QD which involve the same conduction electron in its ground or excited state 
experience the same nuclear field. This property is clearly evidenced by the abrupt cancellation of the nuclear field $B_{n}$ in magnetic field sweeps which take place accordingly at different or identical critical fields, see Fig.26. Note that the coexistence of different excitonic states in the same QD in a PL spectrum results from the random capture of excitons and individual charges under quasiresonant excitation conditions. In the measurement of Fig. 26 the spin-polarized positive trions $X^{+}$attract a photo-created electron during their lifetimes and transform into neutral biexcitons $2 X^{0}$ with no polarization. For QDs inserted in a Schottky structure such effect can be favored by tuning the gate voltage near critical values separating different charge states.

One could expect the coexistence of biexcitons and trions to affect the DNP magnitude. Biexcitons indeed recombine to an exciton state with no specific circular polarization so that the average electron spin driving the nuclear field in Eq. 20 should be reduced. However, the electron-nucleus flip-flop turns out to be largely inhibited for neutral excitons because it couples bright ( $\Downarrow$ or $\downarrow \uparrow)$ to dark excitons ( $\uparrow$ or $\downarrow \Downarrow)$ which are separated by the electron-hole exchange energy $\delta_{0}$. For InAs/GaAs QDs where $\delta_{0} \simeq 0.2-0.5 \mathrm{meV}$ (Urbaszek et al. 2003 ) this would correspond to a magnetic field of about $15 \mathrm{~T}$ to be compensated. The possible contribution of excitons to DNP is therefore quite negligible compared to that of trions under usual experimental conditions. However, in interfacial GaAs QDs for which $\delta_{0} \sim 100 \mu \mathrm{eV}$ the observation of large nuclear polarization in weak fields around $100 \mathrm{mT}$ could still be assigned to flip-flops between neutral exciton states (Gammon et al. 2001). The low DNP rate $T_{1 e}^{-1} \propto \delta_{0}^{-2}$ estimated to $2.5 \mathrm{~s}^{-1}$ was compensated in this case by a vanishing nuclear spin relaxation assumed to be only due to dipolar coupling. More recently, neutral excitons have been proposed to be at the origin of a strong Overhauser effect measured for InP/InGaP QDs at low optical excitation power in a strong magnetic field $B_{z}=6 \mathrm{~T}$ (Chekhovich et al. 2011b) near the dark-bright exciton crossing point.

Even though neutral excitons do not necessarily contribute to DNP they still experience the nuclear field produced by trions (Eble et al. 2006). To some extent this effect can be used to control the intrinsic polarization of neutral excitons in zero magnetic field (Belhadj et al. 2009, Larsson et al., 2011, Moskalenko et al., 2009). The optical orientation of neutral excitons $X^{\mathrm{U}}$ in InAs/GaAs QDs is usually not possible because of the anisotropic electron-hole exchange $\delta_{1} \sim 30 \mu \mathrm{eV}$ which splits the levels into linearly-polarized states. An external field substantially larger than $\delta_{1} / g_{X^{0}} \mu_{\mathrm{B}} \sim 150 \mathrm{mT}$ would be required to restore circularly-polarized eigenstates. As shown in Fig. 27, this can be partially achieved when $X^{+}$trions are formed alternatively with $X^{0}$ excitons under circularly-polarized non-resonant optical pumping, since the former can generate a nuclear field $B_{n}$ reaching several $100 \mathrm{mT}$. Note however that $B_{n}$ is the effective magnetic field experienced by the electron only and de-

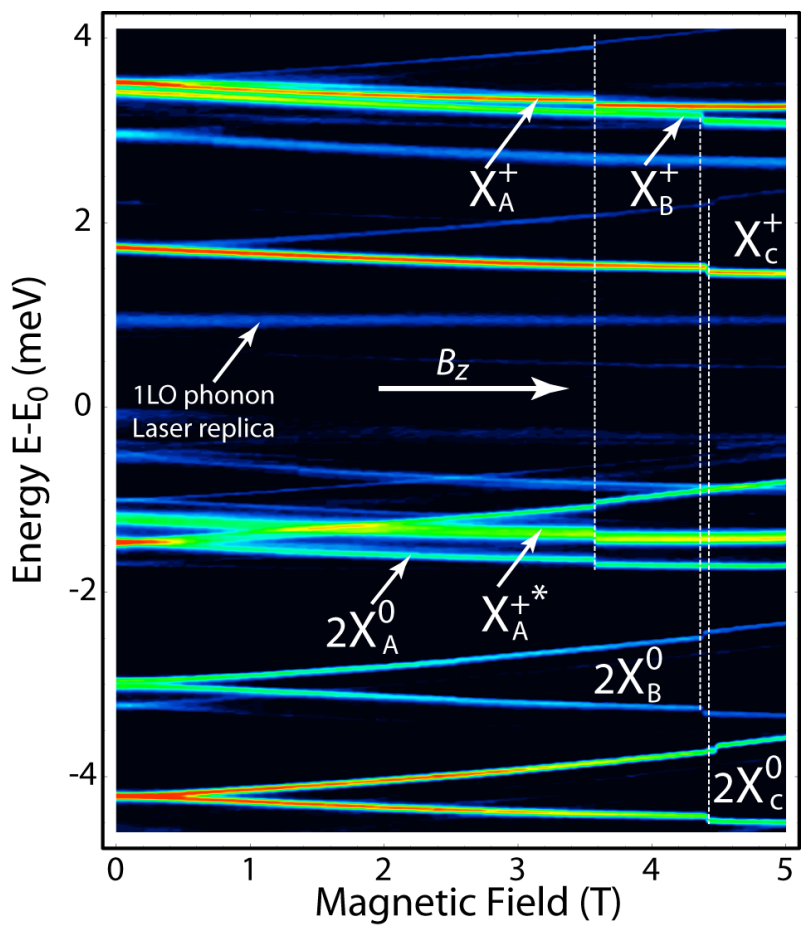

FIG. 26 Density plot of PL spectra of three different QDs (labelled A, B and C) around $E_{0}=1.36 \mathrm{eV}$ as a function of a longitudinal magnetic field under $\sigma^{-}$excitation at about $1 \mathrm{LO}$ phonon energy $\left(37 \mathrm{meV}\right.$ ) above $E_{0}$. The specific critical fields at which the nuclear polarization collapses vary from dot to dot and allow to recognize different excitonic lines originating from the same QD.

duced from its Overhauser shift according to $\hbar \omega_{O S}^{e} / g_{e} \mu_{\mathrm{B}}$. The $X^{0}$ eigenstates are actually determined by the ratio $\eta=\left|\delta_{1} / \hbar \omega_{O S}^{e}\right|$. When an exciton is created with an initial circular polarization $\rho_{c}^{0}$, it experiences quantum beats during its lifetime $\tau_{r}$ which, if $\tau_{r}$ is long enough, results in an average circular polarization $\rho_{c}^{0}\left(1+\eta^{2}\right)^{-1}$ and a linear polarization $\rho_{c}^{0} \eta\left(1+\eta^{2}\right)^{-1}$ of the emitted PL (Dzhioev et al. 1998a). This effect is demonstrated in Fig. 27(b), with notably a substantial conversion of the initial circular polarization into a linear polarization when the magnitude of the Overhauser shift increases with the excitation power and becomes comparable to $\delta_{1}$.

\section{Nuclear spin polarization under resonant optical excitation}

To investigate the coupled electron-nuclear spin dynamics under resonant excitation sophisticated pump probe techniques (Chekhovich et al. 2010a, Greilich et al. 2007), photocurrent experiments (Klotz et al. 2010) or schemes with spectator states (Kloeffel et al. 2011) have been developed. These experiments have uncovered original nuclear pumping cycles, not accessible under non-resonant excitation conditions. In Sec.V.C.1 

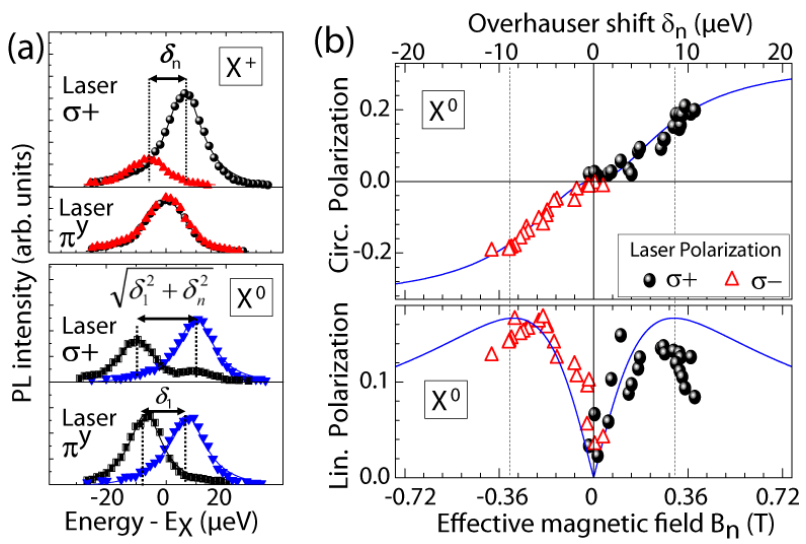

FIG. 27 (a) PL spectra of $X^{0}$ and $X^{+}$from the same InAs/GaAs QD in zero external magnetic field under the same experimental conditions, yet measured in $\left(\pi^{x}, \pi^{y}\right)$ and $\left(\sigma^{+}, \sigma^{-}\right)$basis respectively. The same Overhauser shift $\delta_{n} \equiv$ $\hbar \omega_{O S}^{e}$ generated under $\sigma^{+}$circular polarization is evidenced for both $X^{0}$ and $X^{+}$. (b) Evolution of circular (top) and linear (bottom) polarization of $X^{0} \mathrm{PL}$ as a function of the measured Overhauser shift which increases in absolute value when the power of the $\sigma^{ \pm}$excitation increases. Solid lines follow the simple theoretical analysis given in Belhadj et al. (2009))

an efficient electron spin initialization scheme is presented, that results in DNP via the Overhauser effect. In Sec.V.C.2 we will describe how a resonant laser can drive an optically forbidden transition, where photon absorption is accompanied by a simultaneous spin flip of an electron and a nuclear spin. The DNP achieved in these original schemes is based on the flip-flop term $\propto\left(\hat{I}_{+} \hat{S}_{-}^{e}+\hat{I}_{-} \hat{S}_{+}^{e}\right)$ in Eq. (6). In Sec. V.C.3 resonant experiments will be presented that involve nuclear polarization build-up that does not require the simultaneous spin-flip of a carrier spin, i.e. that involves non-collinear terms of the form $\propto \hat{I}_{x} \hat{S}_{z}^{e}$, paving the way for novel nuclear spin control schemes.

\section{Overhauser effects under resonant optical excitation}

An original cycle for efficient DNP generation through resonant optical pumping has been developed by Kloeffel et al. (2011) in small applied longitudinal fields $B_{z}=0.5 \mathrm{~T}$. In this scheme the driving laser polarization and more surprisingly it's frequency will define which electron spin state $(\uparrow$ or $\downarrow)$ is initialised, determining the direction of the nuclear spin polarization created via the Overhauser effect. Here an InAs QD is placed in a charge tunable structure with a highly n-doped layer, separated from the dot by a $25 \mathrm{~nm}$ thick GaAs tunnel barrier. In the experiment the bias voltage is set just between the $\mathrm{X}^{0}$ and $\mathrm{X}^{-}$plateaus. The efficient cycle for electron spin initialisation, see Fig. 28, starts with a dot that is unoccupied before laser excitation, as the first electronic state is just above the Fermi sea of electrons. In the experiment the laser polarisation is set to $\sigma^{+}$. Absorption of a photon leads to the formation of neutral exciton $\mathrm{X}^{0}$ state $|\Uparrow \downarrow\rangle$. The Coulomb attraction of the hole lowers the electronic level just below the Fermi sea, so that after a time $\tau_{\text {in }}$ of typically tens of picoseconds an electron tunnels into the dot. Pauli exclusion determines the incoming electron to be in the $|\uparrow\rangle$ state and the charged exciton $\mathrm{X}^{-}$state $|\Uparrow \downarrow \uparrow\rangle$ is formed. The $\mathrm{X}^{-}$does not polarize the nuclei efficiently since the two electrons form a singlet and the coupling of the unpaired hole to nuclear spins is much weaker than for a single electron (see Sec.VII) ${ }^{10}$. This charged exciton exists for its radiative lifetime $\tau_{r} \approx 1 \mathrm{~ns}$, leaving behind a single electron after photon emission. The intensity of the $\mathrm{X}^{-}$emission is a measure for the $\mathrm{X}^{0}$ absorption (Simon et al. 2011). The unpaired electron $|\uparrow\rangle$ left behind interacts with the nuclear spin system. But this interaction is limited in time: in the absence of the hole the electron energy is above the Fermi sea, therefore the electron will tunnel out of the dot within $\tau_{\text {out }}$ of tens of picoseconds. This short tunneling time (i.e. broadened Zeeman levels) limits the correlation time for the electron-nuclear spin interaction, allowing flip-flops between electron and nuclear spins without violating energy conservation. Once the electron has tunneled out, the dot is again unoccupied and another $\sigma^{+}$photon resonant with the $\mathrm{X}^{0}$ state can be absorbed. DNP builds up by going repeatedly around this cycle.

For a perfectly pure $\mathrm{X}^{0}$ state $|\Uparrow \downarrow\rangle$ one would expect one sharp resonance in absorption. But very surprisingly there is a second resonance in the absorption curve, see Fig. 28(b). This resonance corresponds to the energy of the other $\mathrm{X}^{0}$ bright state $|\Downarrow \uparrow\rangle$, separated from the $|\Uparrow \downarrow\rangle$ exciton, by an energy of $\approx 40 \mu \mathrm{eV}$. Both exciton states are coupled by the anisotropic part of the Coulomb interaction, making the $|0\rangle \longrightarrow|\Downarrow \uparrow\rangle$ transition weakly allowed under $\sigma^{+}$excitation. Once $|\Downarrow \uparrow\rangle$ is created, the dot will receive an additional electron from the Fermi sea, that has to be $|\downarrow\rangle$ due to Pauli exclusion, and the $\mathrm{X}^{-}$state $|\Downarrow \uparrow \downarrow\rangle$ is formed. Radiative recombination leads to $\sigma^{-}$polarized emission, as shown in the top part of Fig. 28(b), and most importantly the electron spin is now $|\downarrow\rangle$ interacting with the nuclei, leading to nuclear spin polarization that has the opposite direction as compared to the previous case. Therefore, the sign of the electron polarization and hence the nuclear polarization can be tuned finely by varying the excitation laser energy (Kloeffel et al., 2011), as shown in Fig.28(b). The DNP generation as a function

10 It is experimentally confirmed that the Ising like term $\propto \hat{I}_{z} \hat{S}_{z}$ is one order of magnitude weaker for heavy holes than for conduction electrons. In addition, as discussed in Sec.VII the interaction of hole spins with nuclei can be strongly anisotropic, see Eq. 33 and discussion in (Fischer et al. 2008 Testelin et al. 2009). As a result, the amplitude of the terms responsible for hole spin - nuclear spin flip flops strongly depend on the degree of valence band mixing and can therefore be several orders of magnitude weaker than for electrons and eventually tend to zero for pure heavy hole states. 

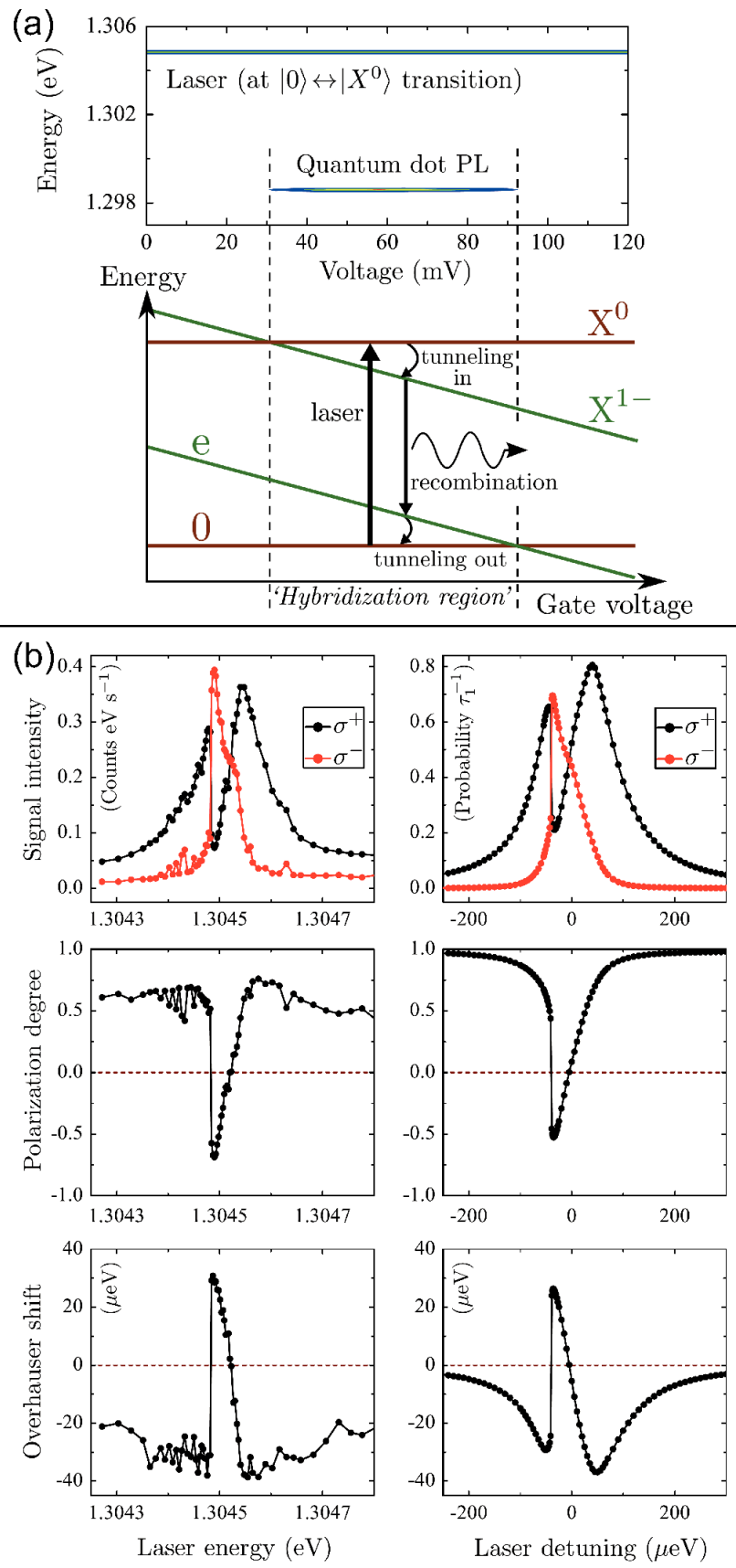

FIG. 28 (a) Top: Photoluminescence (PL) at $4.2 \mathrm{~K}$ from a single $\mathrm{QD}$ versus applied bias driven with excitation at $\mathrm{X}^{0}$ energy. $\mathrm{X}^{-} \mathrm{PL}$ appears in a narrow range of voltage. Bottom: Energy dependence versus bias for the QD vacuum state $|0\rangle$ and the single electron state $|e\rangle$, showing a crossing where the ground state changes. $\mathrm{X}^{0}$ and $\mathrm{X}^{-}$cross at lower bias on account of the hole (Coulomb interaction). For the chosen bias region (Hybridization region), automatic cycling takes place when a laser is tuned to the $|0\rangle \leftrightarrow\left|X^{0}\right\rangle$ transition. (b) Left: Experimentally measured signal intensity, polarization degree and Overhauser shift versus laser energy (detuning) for a $\sigma^{+}$pump and an external field of $+0.5 \mathrm{~T}$ at fixed bias in the center of the hybridization region. Right: Comparison with theory from Kloeffel et al. (2011) of detuning shows strong non-linearities as the transition energies themselves change with each nuclear spin flip, driving the transitions towards or away from the laser energy as described in detail in the model developed by Kloeffel et al. (2011). Because of this feedback, the QD transition can be 'pushed' or 'pulled' by changing the laser frequency, a phenomenon even more pronounced in the experiments described in the Sec.V.C.3. The presented resonant excitation cycle is an efficient way of creating a net electron spin polarization that is subsequently transferred to the nuclear spins via the Overhauser effect. The fact that the carrier excitation is resonant has no direct consequences for the DNP build-up process itself (here based on the standard Overhauser effect), in contrast to the experiments described below.

\section{Pumping of Nuclear Spins by Optical Excitation of Spin-Forbidden Transitions}

Experiments carried out on the $\mathrm{X}^{+}$transitions in $\mathrm{InP}$ dots in a longitudinal magnetic field of 2.5T (Chekhovich et al. 2010a) are shown in Fig.29, where the nuclear spin polarization is measured in non-resonant $\mathrm{PL}$ (probe) as a narrow excitation laser is swept across the $\mathrm{X}^{+}$transitions (pump). In these experiments photon absorption is accompanied by an electron-nuclear spin flip-flop. There is a very important difference for the energy balance of the spin flip-flop as compared to non-resonant excitation: the energy for the electron spin flip is now directly provided by the excitation laser, in close analogy to the solid state effect discussed by Abragam (1961) and more recently in the context of QDs by Bracker et al. (2008) and Korenev (2007).

Assuming initially $100 \%$ pure optical selection rules, the hole $|\Downarrow\rangle$ state absorbs a $\sigma^{+}$photon to become $|\Downarrow \Uparrow \downarrow\rangle$, the hole $|\Uparrow\rangle$ state absorbs a $\sigma^{-}$photon to become $|\Downarrow \Uparrow \uparrow\rangle$ and as the electron and hole g-factors are unequal $\left(\left|g_{h}\right|>\right.$ $\left.\left|g_{e}\right|\right)$ the energies of the $\sigma^{+}$and $\sigma^{-}$transitions are different. In this case one expects when scanning a $\sigma^{+}$polarized laser across the Zeeman branches one absorption line and no DNP, as no carrier spin flip takes place. This is in stark contrast to the reported experiments (Chekhovich et al. 2010a): Driving the dot with $\sigma^{+}$laser light results as expected in absorption when in resonance with the $|\Downarrow\rangle \longrightarrow|\Downarrow \Uparrow \downarrow\rangle$ transition but, and this is the first surprise, there is a second resonance at higher energy for the normally spin forbidden transition $|\Downarrow\rangle \longrightarrow|\Downarrow \Uparrow \uparrow\rangle$. The second surprise is that DNP is created for both resonances, with the normally spin-forbidden absorption scheme polarizing nuclear spins even more efficiently.

Optically assisted nuclear spin-flips can occur either in the absorption or the ensuing spontaneous emission. The corresponding absorption/emission cycles, as depicted in Fig.29(c) are:

(1) The $\sigma^{+}$laser energy is tuned to the nominally forbidden $|\Downarrow\rangle \longrightarrow|\Downarrow \Uparrow \uparrow\rangle$ transition that is difficult to saturate due to weak oscillator strength. In a second order 


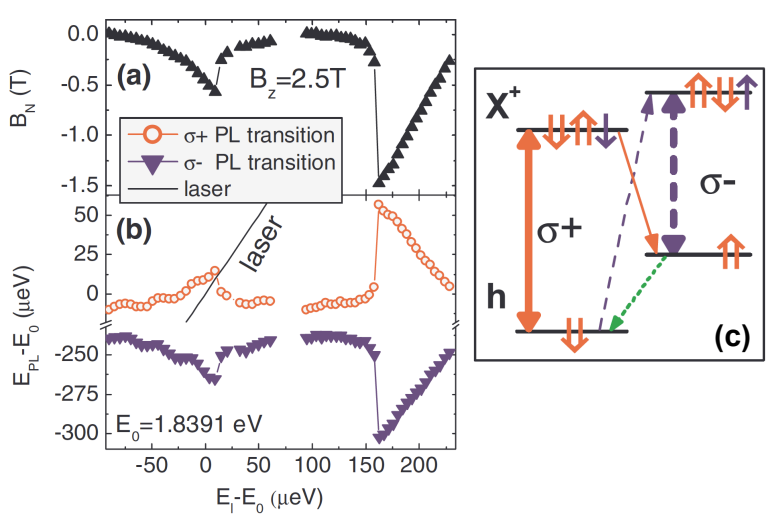

FIG. 29 (a) Overhauser field $B_{N}$ in the dot for $\mathrm{B}_{z}=2.5 \mathrm{~T}$ as a function of laser energy (b) PL transition energy as a function of laser energy (c) Energy level diagram of a positively charged dot in a magnetic field $\mathrm{B}_{z}$. Long thick arrows show "allowed", thin arrows "forbidden" optical transition (Chekhovich et al. 2010a).

process, $\sigma^{+}$photon absorption assisted by a flip of the electron spin and a nuclear spin results in $|\Downarrow \Uparrow \uparrow\rangle$ formation. Subsequently the spin allowed optical recombination $|\downarrow \uparrow \uparrow\rangle \longrightarrow|\uparrow\rangle$ results in $\sigma^{-}$photon emission. For the pumping cycle to start again, the hole spin has to relax from $|\Uparrow\rangle \longrightarrow|\Downarrow\rangle$, a process that is fast enough for this dot system in strong magnetic fields not to limit the efficiency of the spin pumping cycle (Chekhovich et al. 2010a).

(2) Starting again from $|\Downarrow\rangle$ but tuning the laser to the spin allowed $|\Downarrow\rangle \longrightarrow|\Downarrow \Uparrow \downarrow\rangle$ transition results in strong and easily saturable optical absorption. Re-emission of a $\sigma^{+}$photon at the same energy back to $|\Downarrow\rangle$ is the most likely course of events. But due to the hyperfine interaction, the transition $|\Downarrow \Uparrow \downarrow\rangle \longrightarrow|\Uparrow\rangle$ becomes weakly allowed, when assisted by a electron-nuclear spin flip-flop. The final step of this cycle relies again on hole spin relaxation $|\uparrow\rangle \longrightarrow|\Downarrow\rangle$. Completing either one of these two cycles once results in lowering the nuclear spin $z$ projection by 1 . To maximize the generated DNP, the rates characterizing the optical absorption, emission and the resident hole spin flip can be optimized by changing the applied field $\mathrm{B}_{z}$ and/or the laser intensity. Cycle (2) starting with an allowed optical transition saturates already at low pumping power, whereas for cycle (1) the absorption assisted by the hyperfine interaction continues to grow in strength when increasing the laser power. This results in more efficient nuclear spin pumping at high laser powers using the cycle (1), as confirmed by the experiments as well as the theoretical model detailed in Chekhovich et al. (2010a).

\section{Locking of quantum-dot resonances to an incident laser}

Several groups have observed that the textbook-like Lorentzian absorption line shape that one expects as a spectrally narrow single-mode laser field is scanned across the QD resonance gets strongly modified at magnetic fields $B_{z}$ exceeding 1 Tesla. As the laser is tuned within $\sim 2$ linewidths $(\Delta \nu)$ of the $\mathrm{QD}$ resonance, the absorption abruptly turns on. When the laser is further scanned across the resonance (in either direction), the absorption strength remains close to its maximum value for a laser detuning that can exceed $\pm 10 \Delta \nu$, see Fig. 30 .
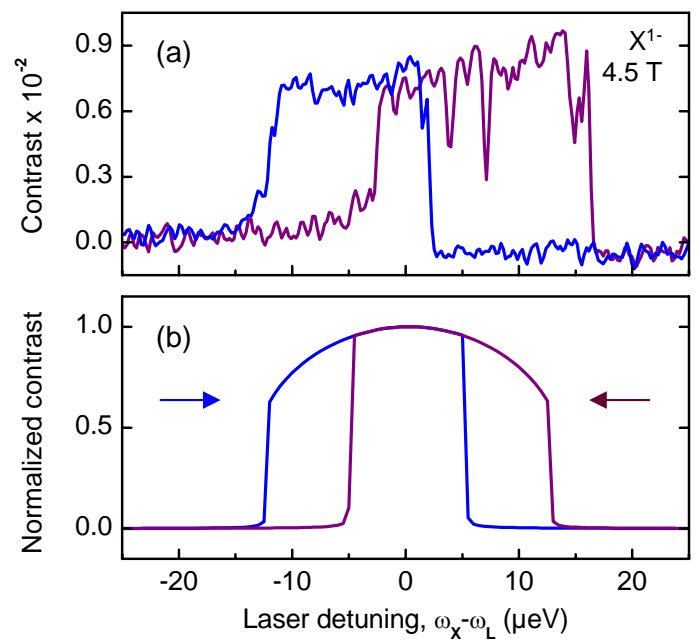

FIG. 30 Comparison of the dragging spectra of the blueshifted Zeeman branch for the $\mathrm{X}^{-}$trion as a function of laser detuning for a sample with $25 \mathrm{~nm}$ thick tunnel barrier (a) and the prediction of the rate equation 28 (b) for the following parameters: $N=3.2 \cdot 10^{4}$, where $N$ is the number of nuclear spins, $\tilde{A}^{i}=120 \mu \mathrm{eV} / N, B_{z}=4.5 \mathrm{~T}, A_{\mathrm{nc}}^{i}=0.012 \tilde{A}^{i}$, $\hbar \Gamma=0.58 \mu \mathrm{eV}, \Omega_{\mathrm{L}}=0.7 \Gamma$, step size $\Delta \delta=0.23 \mu \mathrm{eV}$ and dwell time $t_{c}=0.2 \mathrm{~s} .($ after Högele et al. $(2012)$ ).

Latta et al. (2009) and Högele et al. (2012) have carried out a detailed experimental study of this so-called dragging effect in charge tunable InAs/GaAs QDs (Warburton et al. 2000). The dependence on the laser intensity, laser (or gate voltage) scan-speed and the electron spin-relaxation (co-tunneling) rate have been mapped out. Their findings demonstrate that dragging is a consequence of nuclear spin polarization that enables locking of the QD resonance to the incident probe laser field frequency. Remarkably, for any given (linear or circular) laser polarization, nuclear spin polarization is bidirectional, allowing the combined electron-nuclear spin system to track the changes in laser frequency dynamically on both sides of the resonance. This latter observation suggests that the underlying mechanism is not related to optical pumping of QD electron spin, that is used to explain quasi-resonant DNP experiments for which the flip-flop term of the form $\propto\left(\hat{I}_{+} \hat{S}_{-}^{e}+\hat{I}_{-} \hat{S}_{+}^{e}\right)$ in $\hat{H}_{\mathrm{hf}}^{\mathrm{fc}}$ from Eq. 3 dominates.

Experiments carried out on different charge-tunable 
structures show that any QD transition that has an electron spin decay rate $\lesssim 10^{7} s^{-1}$ will exhibit some degree of dragging. In particular it has been shown that $\mathrm{X}^{0}, \mathrm{X}^{-}$and $\mathrm{X}^{+}$transitions in self-assembled QD samples grown in different laboratories under different conditions, exhibit dragging both in Faraday and Voigt geometries. There is however, a striking difference in the dragging profile of the blue-shifted and the red-shifted Zeeman transition of a neutral (or singly charged) QD: in contrast to the blue-Zeeman transition which exhibits the symmetric flat-top response with maximal absorption described earlier (shown in Fig. 30 and in Fig.6(d)), nuclear spin polarization in the red Zeeman transition ensures that the QD resonance is pushed away from the incident laser frequency. In addition, the line shape is asymmetric with respect to the laser (or gate voltage) scan direction. Before we present an explanation of these features, we highlight the general characteristics of dragging, determined by examining the blue trion transition.

Figure 31(a) shows the two-dimensional (2D) map of resonant absorption (resonance fluorescence in Fig. 31(b)) of the blue-shifted trion transition as a function of laser frequency and gate voltage $V_{\mathrm{g}}$ for two different sample structures exhibiting radically different ranges of the co-tunneling rate $\kappa$ (see section II.E for an introduction to co-tunneling). For a QD that is separated from the Fermi sea by a $25 \mathrm{~nm}$ GaAs barrier (Fig.31(a)), the co-tunneling rate ranges from $>10^{9} s^{-1}$ at the edge of the charging plateau to $\sim 10^{6} s^{-1}$ in the center. Consequently, we observe that the bi-directional dragging effect is strongest in the plateau center and is completely suppressed at the edges $\left(V_{\mathrm{g}} \sim 160 \mathrm{mV}\right.$ and $\sim 260 \mathrm{mV}$ in Fig.31(a), and $V_{\mathrm{g}} \sim 220 \mathrm{mV}$ and $\sim 320 \mathrm{mV}$ in Fig.31(b)), where the electron spin orientation is completely randomized due to the spin flip assisted co-tunneling introduced in Sec.II.E In Fig.31(a), each horizontal cut is obtained by scanning the gate voltage for a fixed laser frequency; red (blue) bars show data obtained by scanning the gate voltage such that the detuning $\Delta \omega=\omega_{\mathrm{X}}-\omega_{\mathrm{L}}$ decreases (increases), where $\omega_{\mathrm{X}}$ is the frequency of the QD transition and $\omega_{\mathrm{L}}$ is the laser frequency. Figure 31(b) on the other hand, shows the resonance fluorescence map, obtained by scanning the single-mode laser frequency at a fixed gate voltage, for a QD separated from the Fermi sea by a $35 \mathrm{~nm}$ tunnel barrier. Due to strong electron spin pumping in the center, dragging in this case is restricted to the edges of the single-electron charging plateau. The overall range for dragging is $\sim 30 \mu \mathrm{eV}$ for $B_{z}=5 \mathrm{~T}$, which has to be compared with the theoretical absorption linewidth in the radiative limit $\hbar / \tau_{r} \approx 1 \mu \mathrm{eV}$. The range of dragging depends on the laser Rabi frequency $\Omega_{\mathrm{L}}$; while some degree of dragging is observed for $\Omega_{\mathrm{L}}$ ranging from $\sim 0.3 \Delta \nu$ to $3 \Delta \nu$, the maximum width is obtained at saturation intensity.

Experiments carried out on QDs in all samples showed that the dragging width (in energy) increases sub-linearly with $B_{z}$ beginning at $\sim 1 \mathrm{~T}$ (Högele et al., 2012). This re-

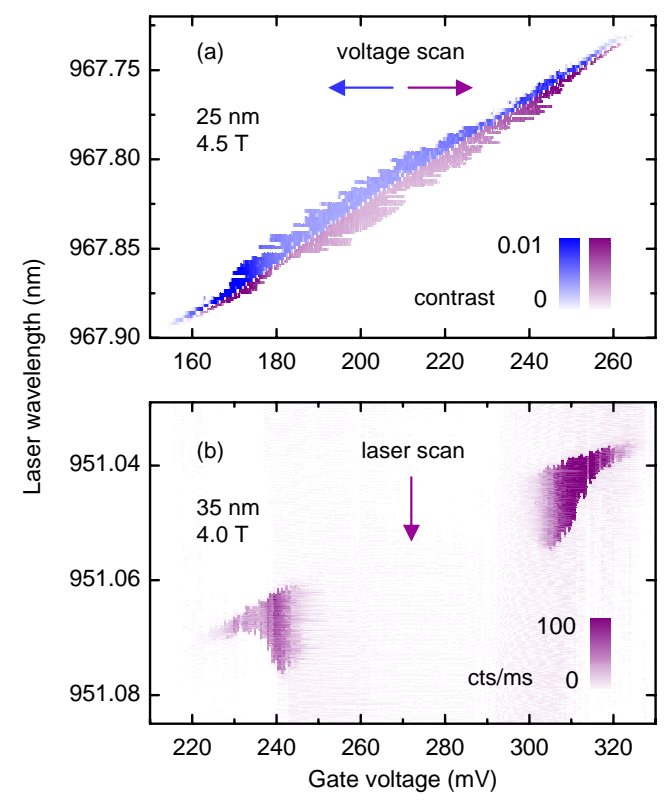

FIG. 31 Differential transmission as a function of gate voltage showing a dragging plateau for a charge tunable sample with a tunnel barrier thickness of (a) $25 \mathrm{~nm}$ at $B_{z}=4.5 \mathrm{~T}$ and (b) as (a), but measured in resonance fluorescence for tunnel barrier thickness $35 \mathrm{~nm}$ at $B_{z}=4.0 \mathrm{~T}$ sample.

sult is at first sight unexpected, given that the maximum Overhauser field that builds up as a result of dragging is a factor of 5 smaller than the external field: therefore, a compensation of the electron Zeeman splitting due to $B_{z}$ by the built-in Overhauser field $B_{n}$ is not taking place. If the mechanism for DNP was based on Fermi-contact hyperfine interaction $\hat{H}_{h f}^{f c}$ of Eq.3. we would have expected the polarization rate at high $B_{z}$ to be suppressed due to energy non-conservation of the hyperfine induced spin flip-flop processes. We emphasize here that in contrast to the quasi-resonant experiments, the electron spin coherence time in resonant experiments is an order of magnitude longer. Since the exchange interaction with the Fermi reservoir needs to be suppressed to observe dragging, electron-nuclear flip-flop processes accompanied by a co-tunneling event only lead to weak directional Overhauser effect. Similar arguments apply to spin-flip spontaneous Raman scattering leading to reverse Overhauser effect (Latta et al., 2009).

The experimental observations therefore suggest that DNP based on hyperfine flip-flop interaction and electron spin-pumping are unlikely to explain the dragging effect. Noting this difficulty, Yang and Sham (Yang and Sham, 2010) proposed that the presence of a small non-collinear hyperfine coupling of the form given in Eq. 15 between the heavy-hole and the nuclear spins would lead to symmetric bi-directional nuclear spin polarization. Below it is argued that non-collinear hyperfine coupling is a key ingredient to explain dragging. The strength of the heavy-hole to nuclear spin coupling does rely on heavyhole light-hole mixing, see also Sec.VII of this review. 
In the sample material used by Latta et al. (2009) and Högele et al. (2012), however, experimentally determined values of hole-hyperfine interaction would suggest that the resulting nuclear spin-flip rates would be an order of magnitude too small to explain the relative insignificance of the directional reverse Overhauser effect. In addition, the large apparent variation in heavy-light-hole mixing in QDs as indicated by the measured in-plane hole $g$-factors would suggest that dragging features would change appreciably from $\mathrm{QD}$ to $\mathrm{QD}$, if the mechanism was due to hole-hyperfine interaction, described in detail in Sec.VII This is in contradiction with the experimental observations. Moreover, recent experiments (Latta et al., 2011b described in Sec.VI.C demonstrate that non-collinear hyperfine interaction between the electron and the nuclei plays a significant role in determining QD nuclear spin dynamics even in the absence of optically generated holes. As an alternative origin to the proposed coupling of holes to nuclei, this important noncollinear interaction with electrons can be induced by large quadrupolar fields in strained self-assembled QDs (introduced in Sec.III.B) which ensure that nuclear spin projection along $B_{z}$ is not a good quantum number. We recall that an effective non-collinear interaction between the electron in the dot and the nuclei of the simple form

$$
\hat{H}_{h f}^{n c}=\sum_{i} A_{\mathrm{nc}}^{i} \hat{I}_{x}^{i} \hat{S}_{z}^{e}
$$

for highly strained self-assembled QDs was introduced by Huang and $\mathrm{Hu}$ (Huang and $\mathrm{Hu}, 2010)$. Here, $A_{\mathrm{nc}}^{i}=$ $A^{\text {nc }} / N$, and $A^{\text {nc }}$ is the non-collinear counterpart of $2 \tilde{A}$ in $\hat{H}_{h f}^{f c}$ of Eq. (6).

Our next target is to understand the physical origin of this effective non-collinear coupling. Let us recall (see section III.B that the quadrupolar interaction Hamiltonian for a nuclear spin with strain axis (i.e. the principal axis of the electric field gradient) tilted by an angle $\theta$ from the $z$-axis in the $x z$ plane in the limit of small angles (compare with Eq.9), can be written as:

$$
\begin{aligned}
\hat{H}_{\mathrm{Q}} & =B_{Q}\left[\hat{I}_{z}^{2} \cos ^{2} \theta-\frac{I(I+1)}{3}\right. \\
& \left.+\left(\hat{I}_{z} \hat{I}_{x}+\hat{I}_{x} \hat{I}_{z}\right) \sin \theta \cos \theta\right] .
\end{aligned}
$$

The first two terms are very small corrections to the energy and will not be considered in the following. We focus our attention on the term $\propto\left(\hat{I}_{z} \hat{I}_{x}+\hat{I}_{x} \hat{I}_{z}\right)$, which can be treated as a perturbation to the nuclear Zeeman energy under the experimental conditions $B_{z}=5 T \gg B_{Q}$, and apply a Schrieffer-Wolff (SW) transformation (Schrieffer and Wolff, 1966). When this transformation is applied to the $\sum_{i} A_{i} S_{z} I_{z}$ term of the Fermi-contact hyperfine interaction Hamiltonian $\hat{H}_{h f}^{f c}$, we obtain

$$
\hat{H}_{h f-q u a d}=\sum_{i} \tilde{A}_{\mathrm{nc}}^{i} \hat{S}_{z}^{e}\left[\hat{I}_{x}^{i} \hat{I}_{z}^{i}+\hat{I}_{z}^{i} \hat{I}_{x}^{i}\right]
$$

with $\tilde{A}_{\mathrm{nc}}^{i}=A^{i} \frac{B_{Q}^{i}}{\omega_{Z}^{n}} \sin 2 \theta^{i}$. In $\hat{H}_{h f-q u a d}$ we have only kept the terms that describe processes which leave the electron spin-state unchanged, since contributions that flip the electron spin will have a negligible contribution at high external fields $B_{z}$ as they are energetically forbidden.

The transformed hyperfine interaction Hamiltonian reads $\hat{H}_{h f}=\hat{H}_{h f}^{f c}+\hat{H}_{h f-q u a d}$. A calculation of $B_{Q}^{i}$ and $\theta_{i}$ for $\mathrm{In}_{0.7} \mathrm{Ga}_{0.3}$ As QDs has been carried out where atomistic strain and nuclear quadrupolar distributions are extracted over a relaxed structure (Högele et al., 2012). These calculations yield an average value $A_{\mathrm{nc}}^{i} \simeq 0.07 A^{i}$. Anionic As nuclei dominate the non-collinear hyperfine coupling term by contributing $75 \%$ to the average value; the residual $25 \%$ are due to the cations, where the contribution of In nuclei due to their large nuclear spin of $9 / 2$ is a factor of 10 larger than that of Ga nuclei.

We emphasize that $\hat{H}_{h f-q u a d}$ differs from the noncollinear hyperfine term of Eq. (15) we introduced earlier, since it does not allow for a coupling between nuclear spin states with positive and negative spin-projection along the $z$-axis (the difference compared to the $\hat{I}_{x}$ operator is that $\hat{I}_{x} \hat{I}_{z}+\hat{I}_{z} \hat{I}_{x}$ does not couple the $\pm 1 / 2$ states to each other). It could be argued however that even for large $B_{z}$, the dominant role of flip-flop terms of Fermi-contact hyperfine interaction is to induce indirect interaction between the QD nuclei (Latta et al., 2011b) as introduced in Eq.16. the primary effect of this interaction, in the presence of fast optical dephasing of the electronic spin resonance, is to ensure that the nuclear spin population assumes a thermal distribution on timescales fast compared to the polarization timescale determined by $A_{\mathrm{nc}}^{i}$. In this limit, the dynamics due to $\hat{H}_{\text {hyp-quad }}$ in Eq. (24) will be indistinguishable from that described by $\hat{H}_{n c}$ in Eq. 15). Aiming at this stage for a qualitative understanding of the dragging mechanism further analysis is based on the reasonable assumption that the quadrupolar fields in highly strained QDs can give rise to noncollinear hyperfine interaction with effective dynamics of the general form $\hat{I}_{x}^{i} \hat{S}_{z}^{e}$ provided we take $\tilde{A}_{\mathrm{nc}}^{i}=A_{\mathrm{nc}}^{i}$.

The fact that $\hat{H}_{h f}^{n c}$ as defined in Eq. 15 could explain dragging is at first sight surprising since its dominant effect appears to be nuclear spin diffusion. However, a careful inspection shows that the same Hamiltonian also leads to a small polarization term whose direction is determined by the sign of the optical detuning. To explain this, we focus on $\mathrm{X}^{-}$and simplify the physical system by considering only the blue-shifted trion transition. For this system, we can write down an effective Hamiltonian:

$$
\begin{aligned}
\hat{H}_{d r a g} & =\Delta \omega_{\mathrm{L}} \hat{\sigma}_{t t}+\omega_{\mathrm{Z}}^{\mathrm{n}} \hat{I}_{z}+\sum_{i} 2 \tilde{A}^{i} \hat{I}_{z}^{i} \hat{S}_{z}^{e} \\
& +\Omega_{\mathrm{L}}\left(\hat{\sigma}_{t \uparrow}+\hat{\sigma}_{\uparrow t}\right) \\
& +\sum_{i} A_{\mathrm{nc}}^{i} \hat{S}_{z}^{e} \hat{I}_{x}^{i} .
\end{aligned}
$$

with $\hat{\sigma}_{e g}=|e\rangle\langle g|$. Here, $|t\rangle$ and $|\uparrow\rangle$ denote the excited trion state and the single-electron spin ground states of 
$\mathrm{X}^{-} ; \Delta \omega_{\mathrm{L}}=\omega_{t}-\omega_{\mathrm{L}}$ is the optical detuning with respect to the bare trion resonance at $\omega_{t}$ and $\Omega_{\mathrm{L}}$ is the Rabi frequency of the laser field. The complete system dynamics is given by a master equation for the reduced density operator $\rho$ involving coherent dynamics due to $\hat{H}_{\text {drag }}$ and a dissipative evolution given by the Liouvillian $\mathcal{L}(\rho)=0.5 \Gamma\left(2 \hat{\sigma}_{\uparrow t} \rho \hat{\sigma}_{t \uparrow}-\hat{\sigma}_{t t} \rho-\rho \hat{\sigma}_{t t}\right)$, with $\Gamma$ denoting the spontaneous emission rate of the trion state.

In Eq. 25), we have neglected the flip-flop terms of the Fermi-contact hyperfine interaction. To justify this assumption, we consider the limit of a large external magnetic field where $\omega_{\mathrm{Z}}^{\mathrm{e}} \gg \Omega_{\mathrm{L}} \sim \Gamma$. If we in addition assume $\omega_{\mathrm{Z}}^{\mathrm{n}} \gg \Omega_{\mathrm{L}} \sim \Gamma$, the nuclear spin-flip processes described by the last term in Eq. (25) are energetically forbidden to first order in perturbation theory. Eliminating these terms by a SW transformation we arrive at the following correction terms due to the non-collinear hyperfine interaction to the laser-trion coupling

$$
\hat{H}_{n c-l a s e r}=i \sum_{i} \frac{\Omega_{\mathrm{L}} A_{\mathrm{nc}}^{i}}{2\left(\omega_{\mathrm{Z}}^{\mathrm{n}}-\tilde{A}^{i}\right)}\left(\left(\hat{\sigma}_{\uparrow t}-\hat{\sigma}_{t \uparrow}\right) \hat{I}_{y}^{i}\right) .
$$

Application of the same SW transformation to the Liouvillian term leads to nuclear-spin-flip assisted spontaneous emission terms with rate $\simeq \Gamma\left(A_{\mathrm{nc}}^{i} / 4 \omega_{\mathrm{Z}}^{\mathrm{n}}\right)^{2}$. The corresponding terms for spin-flip Raman scattering processes arising from the $\hat{H}_{h f}^{f c}$ take place at a rate $\simeq$ $\Gamma\left(2 \tilde{A}^{i} / 4 \omega_{\mathrm{Z}}^{\mathrm{e}}\right)^{2}$; given that $\omega_{\mathrm{Z}}^{\mathrm{e}} \simeq 1000 \omega_{\mathrm{Z}}^{\mathrm{n}}$ and $A_{\mathrm{nc}}^{i} \simeq 0.07 \tilde{A}^{i}$, we conclude that the latter processes will take place at a rate that is $\sim 1000$ times slower.

To obtain a qualitative understanding of the coupled electron-nuclear-optical dynamics, we consider the energy level diagram in Fig.32, which shows a ladder of two-level quantum systems coupled by nuclear spin-flip processes. Here we adopt a mean-field description of the nuclear spins by neglecting the quantum fluctuations in the Overhauser field $\left(I_{z}=\left\langle\hat{I}_{z}\right\rangle\right)$. We also make the assumption that the electron couples equally to all nuclear spins with a coupling strength $\tilde{A}^{i}=\tilde{A} / N$. For a given nuclear spin polarization $I_{z}$ we can label the two level system by the states $\left|\uparrow, I_{z}\right\rangle$ and $\left|t, I_{z}\right\rangle$, where $|t\rangle$ stands for the $X^{-}$trion formed by an individual hole and two electrons in a spin-singlet state. The excited states $\left|t, I_{z}\right\rangle$ do not couple to the nuclear spins (we neglect the hole Overhauser field) and differ by an energy $\omega_{\mathrm{Z}}^{\mathrm{n}}$. The ground states which couple to the nuclear spins differ by an energy $\omega_{\mathrm{Z}}^{\mathrm{n}}-\tilde{A} / N$. The strong direct laser coupling and spontaneous emission processes are depicted in black, whereas the hyperfine assisted processes are depicted in red.

The diagonal spontaneous emission processes taking place at rate $\Gamma_{+}=\Gamma_{-}=\Gamma_{\text {diff }}=\Gamma\left(A_{\mathrm{nc}}^{i} / 2 \omega_{\mathrm{Z}}^{\mathrm{n}}\right)^{2}$ cause non-directional nuclear spin diffusion, limiting the range of achievable DNP. The transition rate associated with hyperfine-assisted laser coupling on the other hand is

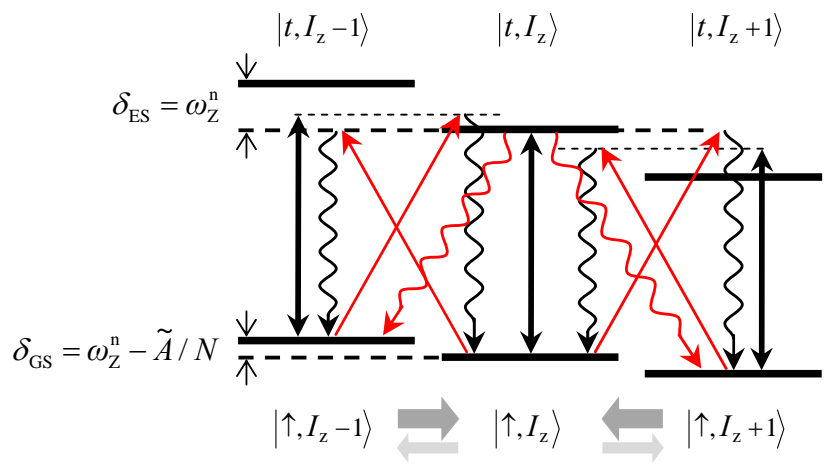

FIG. 32 Energy level diagram depicting the nuclear spin flip assisted transitions for the $X^{-}$trion higher energy Zeeman branch in a finite magnetic field applied along the growth direction $z$ : a resonant laser field couples dipole allowed and dipole forbidden transitions (straight and diagonal arrows, respectively) of the trion-nuclear spin manifold. The lower states of the manifold are electron spin-up states $|\uparrow\rangle$ split by the sum of nuclear Zeeman energy and the Overhauser field, $\delta_{\mathrm{GS}}=\omega_{\mathrm{Z}}^{\mathrm{n}}-\tilde{A} / N$, according to their nuclear spin projection $I_{z}$ along $z$. The upper states are $X^{-}$trion states (an individual hole and two electrons in a spin-singlet state) split by the nuclear Zeeman energy through $\delta_{\mathrm{ES}}=\omega_{\mathrm{Z}}^{\mathrm{n}}$. Nuclear spin polarization occurs through spin-flip assisted diagonal transitions (diagonal arrows) followed by spin preserving radiative decay (wavy arrows). Finite laser detunings lead to an imbalanced competition between the bidirectional nuclear spin diffusion processes within the manifold (horizontal arrows): the coupled trion-nuclear spin system reaches steady state by locking to the laser resonance.(after Högele et al. (2012)).

given by

$$
\begin{aligned}
W_{ \pm}\left(I_{z}\right)= & \left(\frac{\Omega_{\mathrm{L}} \tilde{A}_{\mathrm{nc}}^{i}}{4\left(\omega_{\mathrm{Z}}^{\mathrm{n}}-\tilde{A}^{i}\right)}\right)^{2} \\
& \frac{\Gamma}{4\left(\Delta \omega_{\mathrm{L}}-\tilde{A}^{i}\left(I_{z} \pm 1\right) \mp \omega_{\mathrm{Z}}^{\mathrm{n}}\right)^{2}+\Gamma^{2}}
\end{aligned}
$$

A remarkable feature of $W_{ \pm}\left(I_{z}\right)$ is its dependence on the sign of the laser detuning entering through the effective optical detuning $\delta=\Delta \omega_{\mathrm{L}}-\tilde{A}^{i}\left(I_{z} \pm 1\right) \mp \omega_{\mathrm{Z}}^{\mathrm{n}}$ : when the incident laser field is red (blue) detuned, the transition rate $W_{+}\left(I_{z}\right)\left(W_{-}\left(I_{z}\right)\right)$ dominates over $W_{-}\left(I_{z}\right)$ $\left(W_{+}\left(I_{z}\right)\right)$ and ensures that the Overhauser field increases (decreases). This directional nuclear spin polarization will on the other hand result in a decrease of $\delta$ from $\Delta \omega_{\mathrm{L}}-I_{z}$ to $\Delta \omega_{\mathrm{L}}-I_{z}-\tilde{A}^{i}\left(\Delta \omega_{\mathrm{L}}-I_{z}+\tilde{A}^{i}\right)$. If initially $I_{z} \ll N / 2$, then DNP will then continue until $\delta \simeq 0$, in consistence with the experimental observations.

To obtain a more quantitative prediction, we consider 
the rate equation ${ }^{11}$ :

$$
\frac{d I_{z}}{d t}=W_{+}\left(I_{z}\right)\left(N / 2-I_{z}\right)-W_{-}\left(I_{z}\right)\left(N / 2+I_{z}\right)-\Gamma_{\operatorname{diff}} I_{z}
$$

which exhibits bistability due to the nonlinear $I_{z}$ dependence of the rates $W_{ \pm}\left(I_{z}\right)$. A comparison between the predictions of this rate equation and experiments is shown in Fig. 30. Figure 30(a) shows two laser scans across the blue Zeeman line of $X^{-}$at $4.5 \mathrm{~T}$ with opposite sweep directions. For both scans, DNP was erased by waiting at a gate voltage of strong co-tunneling for 10 s. A calculation of the differential transmission signal is potted in Fig. 30(b). The experimental findings are qualitatively well reproduced by the theoretical description based on the non-collinear hyperfine coupling.

So far, we have not discussed the red-shifted trion transition. While a laser scan across the blue transition leads to a positive feedback of the nuclear spins to ensure locking condition, a scan across the red Zeeman line will cause an anti-dragging effect. To understand this, we note that the transition rate associated with hyperfineassisted laser coupling in this case is given by

$$
\begin{aligned}
R_{ \pm}\left(I_{z}\right)= & \left(\frac{\Omega_{\mathrm{L}} \tilde{A}_{\mathrm{nc}}^{i}}{4\left(\omega_{\mathrm{Z}}^{\mathrm{n}}-\tilde{A}^{i}\right)}\right)^{2} \\
& \frac{\Gamma}{4\left(\Delta \omega_{\mathrm{L}}+2 \tilde{A}^{i}\left(I_{z} \pm 1\right) \mp \omega_{\mathrm{Z}}^{\mathrm{n}}\right)^{2}+\Gamma^{2}} .
\end{aligned}
$$

The simple sign change in the effective optical detuning from $\delta=\Delta \omega_{\mathrm{L}}-\tilde{A}^{i}\left(I_{z} \pm 1\right) \mp \omega_{\mathrm{Z}}^{\mathrm{n}}$ in Eq. 27) for the blue trion to $\delta=\Delta \omega_{\mathrm{L}}+\tilde{A}^{i}\left(I_{z} \pm 1\right) \mp \omega_{\mathrm{Z}}^{\mathrm{n}}$ renders the exact resonance between the laser field and the trion transition an unstable state. The DNP that ensues in the presence of a small but non-zero $\delta$ will result in nuclear spin flip processes that increase $|\delta|$ resulting in pushing the QD trion transition away from the laser field.

These considerations allow to explain the dragging observed for the blue- and red-shifted transitions (Högele et al. 2012), suggesting that the dragging phenomenon in resonantly driven QD transitions is due to non-collinear electron hyperfine interaction. The main requirement for DNP via resonant laser scattering is the presence of an unpaired electron spin with a long spin-flip-time, either in the initial or the final state of the optical transition. This condition is generically satisfied by all fundamental QD transitions, i. e. the neutral exciton $X^{0}$ as well as the singly negatively and positively charged excitons, $X^{-}$ and $X^{+}$. In all cases, the Overhauser field experienced by the unpaired electron facilitates the feedback that modifies the QD transition energy. Whether or not this feedback leads to resonance seeking or resonance avoiding excitations, as in the blue and red Zeeman branch transitions, respectively, depends on the spin orientation of the electron that couples to the incident laser field.

\footnotetext{
${ }^{11}$ For simplicity we consider a system of spin $\mathrm{I}=1 / 2$ nulcei.
}

4. Preparation of nuclear spin states using periodic pulsed-laser excitation of quantum dot ensembles

Given the large inhomogeneous distribution of QD optical transition energies, we would expect that nuclear spin manipulation in an ensemble of QDs would only be possible either with non-resonant excitation or by resonant short-pulse/broad-bandwidth laser pulses. Even then, sizeable dot-to-dot variations in electron g-factor would make it practically impossible to assess the degree of nuclear spin polarization and it was far from obvious that there could be any signatures of nuclear spin effects beyond electron-spin decoherence in an ensemble setting.

Remarkably, Bayer and co-workers (Greilich et al. 2007) have demonstrated that hyperfine interaction effects are manifest in electron spin dynamics when an ensemble of QDs is driven by periodic short-laser pulsetrains. In their experiments (and as an extension to the experiments discussed in Sect.IV.D), electron spin polarization is generated along the growth $(z)$ direction using circularly polarized laser pulses with a duration of $\tau_{p}=1.5 \mathrm{ps}$ and a repetition rate of $75.6 \mathrm{MHz}$, corresponding to an inter-pulse separation of $T_{R}=13.2 \mathrm{~ns}$. Even at relatively high external (Voigt configuration) magnetic field strength of $B_{x}=6 \mathrm{~T}$, the electron dynamics can be considered to be completely frozen during the optical excitation. Following the excitation/polarization, the electron spins undergo precession around $B_{x}$ and dephase on a timescale given by $T_{2}^{*}<1$ ns: this anticipated dynamics, depicted in the top panel of Fig. 33, is monitored using the Faraday rotation of a second linearlypolarized probe laser pulse with a variable time-delay with respect to the excitation pulse.

When a second pulse is applied at time $T_{D}$ with $T_{D}=$ $T_{R} / k(k \in \mathbb{Z})$, the authors have observed a burst at a time delay of $2 T_{D}$, indicating a revival of the electron spin coherence in a manner reminiscent of spin-echo. The fact that the observed Faraday signal cannot be explained as a simple echo is evident because the spin polarization also recovers before the arrival of the second laser pulse, as well as at $n T_{D}$ with $n>2$. More strikingly, they observed that the bursts continue to appear minutes after the second (delayed) pulse excitation is turned off.

These stunning observations could be explained if the pulsed-excitation protocol leads to a nuclear spin polarization in each dot that ensures that the Larmor frequency is an integer multiple of $2 \pi / T_{D}$ and hence of $2 \pi / T_{R}$. Even as one starts from an ensemble of QDs, each with a different g-factor, a quasi-continuous Overhauser field generated by nuclear spin polarization and intra-dot diffusion could ensure that this condition is satisfied. If the intrinsic nuclear spin lifetime is $\tau_{n}$, then this polarization is maintained for times $\sim \tau_{n}$ even after the pulses are turned off; since recent single QD experiments have revealed nuclear spin polarization decay times exceeding hours (Latta et al. 2011b; Maletinsky et al., 2009), it is no longer surprising to see the memory of the second pulse train survive for minutes time-scales. 


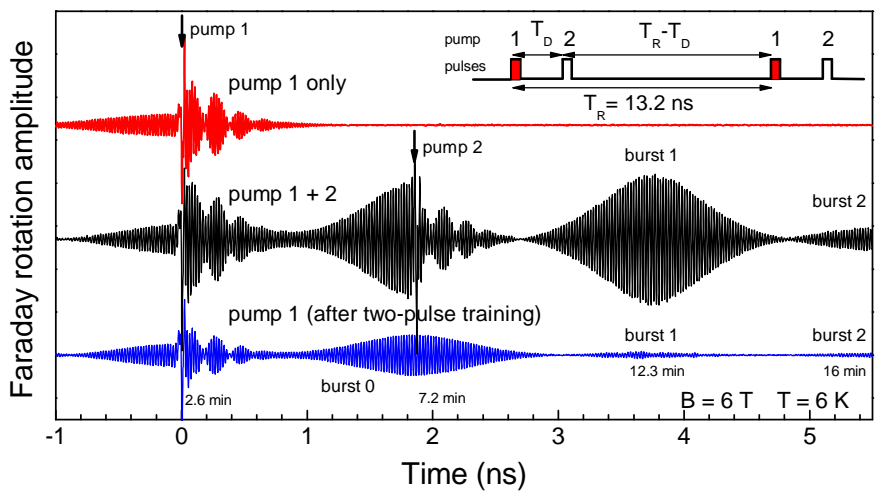

FIG. 33 Faraday rotation signal from an ensemble of singleelectron-charged QDs resonantly excited with a periodic laser pulse train. The data is taken in Voigt geometry at $B_{x}=6 \mathrm{~T}$. The top panel shows the Faraday signal when the ensemble is excited with a single pulse train. Remarkably, excitation with a two-pulse train leads to bursts in Faraday signal at time delays that are integer multiples of the time-delay of the two laser pulses, where electron spins recover a high degree of polarization. Even after the second laser pulse is turned-off, the system continues to produce bursts, suggestive of memory times exceeding minutes (Greilich et al. 2007).

To explain the observed locking effect, we introduce the concept of time-dependent electronic dark-states: recently, it has been shown theoretically that coherent population trapping (CPT) achieved using continuous-wave laser fields satisfying two-photon resonance condition in a single QD in Voigt geometry could lead to preparation of Overhauser field eigenstates with uncertainty that can be as small as the Overhauser field due to a single nuclear spin (Issler et al., 2010). This nuclear spin cooling mechanism by Overhauser field selective CPT relies on pure unbiased optical excitation induced diffusion of QD nuclear spins. The counter-part of CPT electron-spin dark states in the right-hand circularly-polarized $\left(\sigma_{+}\right)$pulsedlaser-train excitation setting is the time-dependent superposition

$$
\left|\psi_{\text {dark-td }}(t)\right\rangle=\frac{1}{\sqrt{2}}\left(\left|\uparrow_{x}\right\rangle-e^{i \phi_{p}} e^{i\left(\omega_{x, j}^{e}-I_{x, j}\right) t}\left|\downarrow_{x}\right\rangle,\right.
$$

where the phase $\phi_{p}$ is set by the pulse-train; this phase together with an Overhauser field $I_{x, j}$ specific to the $j^{t h}$ QD ensure that at times satisfying $t=T_{D}$, the QD electron spin is in state $\left|\downarrow_{z}\right\rangle$, making it dark against optical excitation by a $\sigma_{+}$laser pulse. Provided that the nuclear spin flips are only possible upon excitation of the trion state, once this specific value of $I_{x, j}$ is attained by optical excitation induced unbiased nuclear spin diffusion, nuclear spin dynamics will freeze, ensuring that the dark state condition is satisfied for times that are only limited by $\tau_{n}$.

Given the dominant role non-collinear hyperfine interaction $\hat{H}_{\mathrm{hf}}^{\mathrm{nc}}$ plays in continuous-wave resonant laser induced nuclear spin polarization discussed in Sec.V.C.3. it is natural to consider the origin of the nuclear spin diffusion in the experiments of Greilich et al. (2007). Having seen that at high external magnetic fields, noncollinear hyperfine interaction leads to nuclear spin-flip rates that are more than an order of magnitude stronger than those induced by Fermi-contact flip-flop terms, we argue that nuclear spin diffusion in self-assembled QDs under pulsed-laser excitation should be primarily due to $\hat{H}_{\mathrm{hf}}^{\mathrm{nc}}$. While the directional nuclear spin polarization due to $\hat{H}_{\mathrm{hf}}^{\mathrm{nc}}$ during laser excitation plays a key role in the dragging experiments (Högele et al. 2012), the corresponding processes are negligible in the experiments reported by Greilich et al. (2007) due to the short laserpulse duration; simple considerations show that the probability of a nuclear spin flip during optical excitation (induced by $\left.\hat{H}_{\mathrm{hf}}^{\mathrm{nc}}\right)$ is smaller by a factor $\sim\left(\omega_{z}^{n} \tau_{p}\right)^{2}$ as compared to those taking place during radiative recombination from the trion state. We conclude therefore that unlike continuous-wave excitation, the role of $\hat{H}_{\mathrm{hf}}^{\mathrm{nc}}$ in pulsedlaser excitation is to induce pure unbiased nuclear spin diffusion, allowing the QD nuclei+electron system to find the time-dependent dark state and thereby satisfying the observed phase synchronization condition Greilich et al. 2007).

The reader is referred to Barnes and Economou (2011) and Glazov et al. (2012) for interesting complementary models that help understanding the experimentally observed spin locking effects.

\section{NUCLEAR SPIN DYNAMICS IN QUANTUM DOTS}

A key ingredient for the understanding of the coupled electron-nuclear spin system is the knowledge of the relevant timescales of the dynamics of nuclear spin polarization. This has already become apparent in Sec.V.B.1. where Eq. 19 shows that the maximal nuclear spin polarization in a QD is limited by the ratio of buildup and decay times of the nuclear spins. Many other aspects like the respective roles of nuclear spin diffusion, quadrupolar relaxation and trapped excess QD charges can influence the dynamics of DNP. The buildup time of DNP ( $\left.\tau_{\text {buildup }}\right)$ typically depends on the experimental conditions under which the nuclear spin system is addressed. For an empty QD (free of any charge carriers) the DNP decay time $\left(\tau_{\text {decay }}\right)$ is an inherent property of the isolated nuclear spin system. Furthermore, an experimental determination of $\tau_{\text {decay }}$ is essential for understanding the limits of electron spin coherence in QDs (Merkulov et al. 2002 ), as $\tau_{\text {decay }}$ directly yields the correlation time of the fluctuations of the Overhauser field along the axis in which the nuclei are polarized (see Sect.IV)

\section{A. Dynamics of nuclear spin polarization in low magnetic fields}

The buildup and decay of DNP were studied by several groups (Belhadj et al., 2008, Chekhovich et al., 
2010b; Cherbunin et al., 2009; Makhonin et al., 2010, Maletinsky et al. [2007a; Nikolaenko et al. . 2009) using optical "pump-probe" measurements. The principle of these measurements is illustrated in Fig. 34(a). Short "pump" and "probe" pulses of duration $\tau_{\text {pump }}$ and $\tau_{\text {probe }}$ are used to polarize the nuclear spins and read out the resulting degree of DNP after a waiting time $\tau_{\text {wait }}$. In order to measure the buildup (decay) time of DNP in these experiments, $\tau_{\text {pump }}\left(\tau_{\text {wait }}\right)$ are varied, respectively, while keeping all other experimental parameters fixed.

Figure 34(b) and (c) show examples for buildup and decay curves of DNP obtained with the above-mentioned technique at zero magnetic field in a single InAs QD. In this regime, buildup and decay both follow exponential curves with time-constants $\tau_{\text {buildup }}=9.4 \mathrm{~ms}$ and $\tau_{\text {decay }}=1.9 \mathrm{~ms}$, respectively. The timescale of few tens of ms for optical pumping of nuclear spins at low magnetic fields have additionally been confirmed in different QD systems by Nikolaenko et al. (2009) and Chekhovich et al. 2010b).

Compared to the rather slow timescales of nuclear spin buildup suggested by Gammon et al. (2001) and Maletinsky et al. (2007b) the observed few-ms buildup times are relatively fast. However, these early estimates were based on high-field measurements of DNP, which leads to slower and, due to the nonlinear electronnuclear spin coupling, more complex dynamics of DNP as will be shown in the next section. A further shortening of $\tau_{\text {buildup }}$ compared to earlier studies arises from the strong localization of carriers in self-assembled QDs. Such localization has been shown to be an important ingredient for efficient nuclear spin polarization (Malinowski et al. 2001) as it increases the mean value of the Knight field (Eq. (5) ) and therefore the rate of electron-mediated nuclear spin relaxation in QDs (see Eq. (18)).

\section{Nuclear spin relaxation in semiconductor quantum dots}

While the buildup time of nuclear spin polarization depends on various experimental parameters (like optical excitation intensity etc.), the dynamics of nuclear spin decay is a more intrinsic property of the QD nuclear spin system. Three possible relaxation mechanisms for nuclear spins in semiconductor QDs have been discussed in the past:

- nuclear spin diffusion: Dipolar coupling of like-spins can lead to diffusion of nuclear spin polarization to the surrounding, unpolarized bulk(Abragam, 1961). The terms responsible for nuclear spin diffusion are internuclear flip-flop terms which are energetically only allowed between nuclear spins exhibiting the same levelspacing. In addition to the diffusion mediated by the dipole-dipole interaction, the Fermi-contact hyperfine interaction also leads to nuclear spin diffusion spatially limited to the regions where the electron wave-function is non-vanishing (Klauser et al. , 2006, Latta et al. $2011 \mathrm{~b}$, Paget et al. 1977), see section VI.C

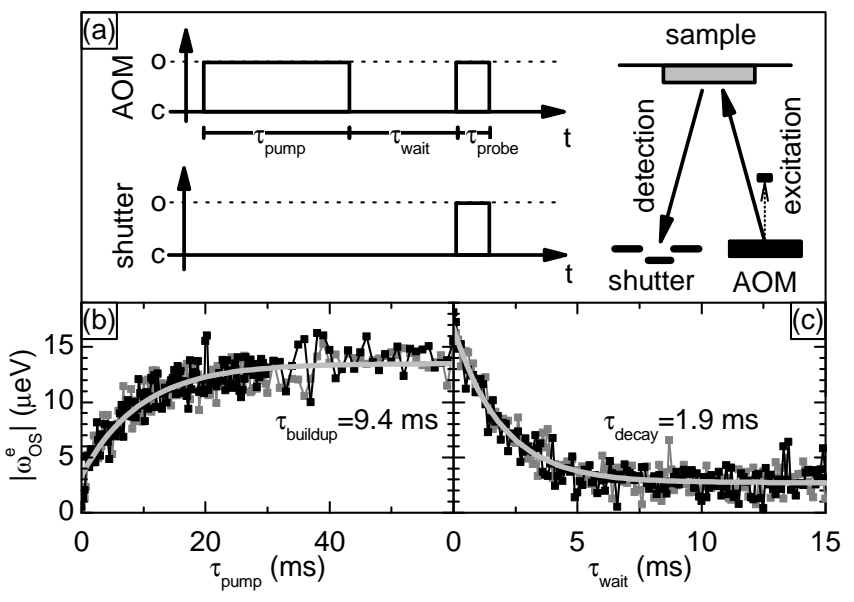

FIG. 34 (a) Schematic of the pulse sequences used in the buildup and decay time measurements of DNP in zero external magnetic field. An optical modulator is used as a fast switch for the PL excitation laser, where o and c denote the open and closed state, respectively. This creates pump (probe) pulses of respective lengths $\tau_{\text {pump }}\left(\tau_{\text {probe }}\right)$, separated by a waiting time $\tau_{\text {wait }}$. A mechanical shutter blocks the pump-induced luminescence from reaching the spectrometer, while letting the probe-induced luminescence pass. (b) Buildup of nuclear spin polarization measured by varying $\tau_{\text {pump }}$ at fixed $\tau_{\text {wait }}(0.5 \mathrm{~ms})$ and $\tau_{\text {probe }}(0.2 \mathrm{~ms})$ for $\sigma^{-}$ $\left(\sigma^{+}\right)$excitation (black and grey, respectively). (c) DNP decay curves obtained by varying $\tau_{\text {wait }}$ at fixed $\tau_{\text {pump }}(50 \mathrm{~ms})$ and $\tau_{\text {probe }}(0.5 \mathrm{~ms})$. Exponential fits (light gray) yield timeconstants $\tau_{\text {buildup }}=9.4 \mathrm{~ms}$ and $\tau_{\text {decay }}=1.9 \mathrm{~ms}$. Adapted from Maletinsky et al. (2007a)

- electron-mediated nuclear spin relaxation: Fluctuations of the spin of electrons occupying a QD typically lead to nuclear spin relaxation through the same processes that allow for nuclear spin pumping in the first place (i.e. electron-nuclear flip-flop interactions). These spin fluctuations can either occur if the charge-state of a given QD is fluctuating, or if a $\mathrm{QD}$ is occupied by an odd number of (unpaired) electrons that are coupled to a nearby charge reservoir, which induces electron spin relaxation through spin-flip co-tunneling introduced in Sec.II.E.

- quadrupolar relaxation of nuclear spins: As introduced in Sec.III.B nuclear spins couple to electric field gradients in the lattice through their quadrupolar moment. Modulation of electric field gradients at nuclear sites can therefore lead to nuclear spin relaxation (Huang and $\mathrm{Hu}, 2010$ ) described in detail in Sec.V.B.2 This modulation can either occur in the form of latticevibrations (phonons) or through QD charge-fluctuations. While phonon-induced quadrupolar relaxation is typically suppressed for temperatures below $20 \mathrm{~K}$ (McNeil and Clark, 1976), quadrupolar relaxation of QD nuclei is relevant even at low temperatures as QD charge fluctuations can be induced either by optical excitation (Paget et al. 2008) or by charges tunneling in and out of a QD. 
Which of these nuclear spin relaxation mechanisms has to be taken into account to explain experimental observations, depends greatly on the QD system under investigation and the experimental parameters. As will be shown below, the more "open" nuclear spin system of QDs formed by interface fluctuations in quantum wells (Gammon et al. 1997) is more likely to exhibit nuclear spin diffusion. In contrast, the nuclear spin system in self-assembled QDs, such as InAs QDs in GaAs, has been shown to be very well isolated from its surrounding (Maletinsky et al., 2009); in fact, the experiments reviewed in this Section demonstrate that nuclear spin relaxation in these systems takes place only when the QD is occupied by an unpaired resident electron.

The exceedingly high nuclear spin relaxation times reported by Greilich et al. (2007), Maletinsky et al. (2009) and Latta et al. (2011b) for self-assembled QDs indicate that nuclear spin diffusion from the QD to its surrounding is negligible in strained InAs/GaAs dots. This is in stark contrast to interfacial QD systems studied by Gammon et al. (1996) or nuclear spin pumping experiments performed by Paget (1982) on shallow donors in GaAs. These "open" nuclear spin systems couple to their bulk surrounding through spin diffusion and therefore exhibit nuclear spin relaxation times on the order of few seconds. A recent study on interfacial QDs however (Nikolaenko et al. , 2009) has shown that even in these systems, nuclear spin diffusion can be inhibited under certain experimental conditions. The exact physical mechanism leading to this suppressed nuclear spin diffusion remains unknown up to now. The different experiments suggest that the dipolar-induced spin diffusion is governed by the chemical contrast, as strongly reduced nuclear spin diffusion measured between two GaAs quantum wells separated by AlGaAs barriers seems to indicate (Malinowski et al., 2001).

In the following we will focus on nuclear spin dynamics in self-assembled QDs, where nuclear spin diffusion from the QD nuclei towards the surrounding matrix is irrelevant and electron-mediated nuclear spin relaxation as well as quadrupolar relaxation can be studied in more detail.

The influence of the QD charge state on nuclear spin polarization has been investigated by Maletinsky et al. (2007b). The experimental arrangement was analogous to the decay measurements presented in Fig. 34(a) with the addition that the QD charge state during the decay time was varied using QD gate electrodes (cf. Sec.II.B). Figure 35(b) shows a comparison between nuclear spin decay for the same QD in the presence (gray line) and absence (black lines) of a single electron. The effect of the QD charge state on DNP lifetime is striking. A further extension of the decay interval (Fig. 35(c)) shows that the nuclear spin lifetime in these QD systems significantly exceeds one hour. This observation is in accordance with earlier measurements of nuclear spin memory time performed by Greilich et al. (2007) on ensembles of self assembled InAs QDs. This near-perfect isolation of the QD

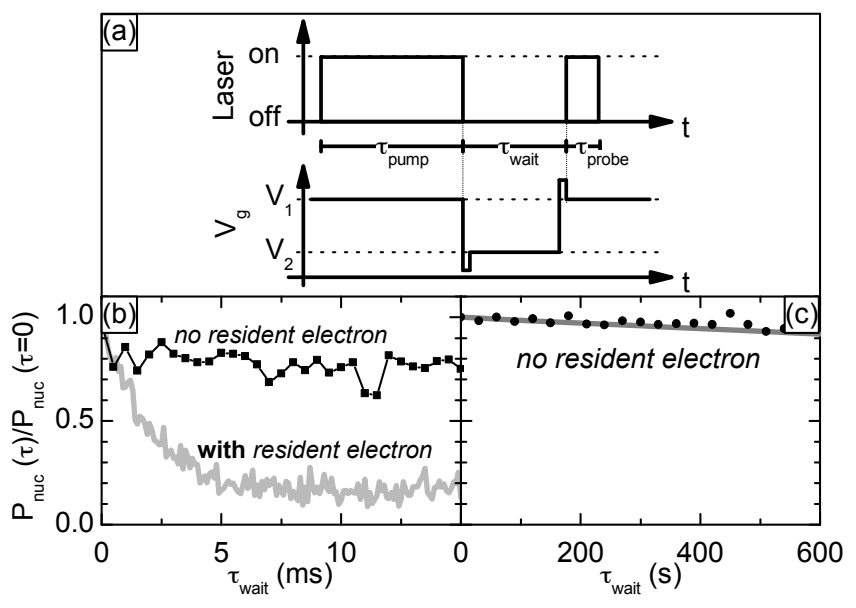

FIG. 35 Measurements in zero external magnetic field.(a) Timing diagram for the gate voltage switching experiment: DNP is established at a gate voltage $V_{1}$, corresponding to the center of the $X^{-1}$ stability plateau. During the period $\tau_{\text {wait }}$, the QD gate voltage is switched to a value $V_{2}$ and the nuclei are left to decay. (b) Measurement of DNP decay, with $V_{2}$ corresponding to the center of the $X^{0}$ stability plateau. The gray (black) data points represent DNP decay under $\sigma^{+}-\left(\sigma^{-}-\right)$ excitation. For comparison, the light gray curve shows the mean of the data presented in Fig.34(b). (c) Same measurement as in (b), but over a longer timescale. The exponential trace (light gray) indicates a decay time constant of 1 hour. Adapted from Maletinsky (2008)

nuclear spin system from its unpolarized environment is a consequence of the strong inhomogeneous quadrupolar shifts of the QD nuclei (c.f. Sec.III.B) as well as the material-mismatch between the QD and its surrounding, which taken together almost completely suppress nuclear spin diffusion out of the QD.

Two mechanisms could lead to the efficient decay of DNP due to the residual QD electron. The first mechanism is caused by the randomization of the electrons spin through co-tunneling to the close-by electron reservoir. Smith et al. (2005) showed that co-tunneling occurs on a timescale of $\tau_{\text {cot }} \approx 3$ ns in the center of the single-electron charging plateau for a structure similar to the one studied in this work. The resulting electron spin depolarization is mapped onto the nuclear spin system via hyperfine flip-flop events. Taking into account the detuning $\omega_{Z}^{e}$ of the two electron spin levels and assume $\omega_{Z}^{e} \gg 1 / \tau_{\text {cot }}$, the nuclear spin depolarization rate can be estimated through Eq. (18) as $T_{1 \mathrm{e}}^{-1} \approx\left(\tilde{A} / N \hbar \omega_{Z}^{e}\right)^{2} / \tau_{\text {cot }}$ (Meier and Zakharchenya 1984). In this expression, $\tau_{\cot }$ plays the role of the hyperfine interaction correlation time in Eq. 18). With $\omega_{Z}^{e}$ assumed constant and equal to half the maximum measured Overhauser shift for a rough estimate, one obtains a nuclear spin depolarization time on the order of $10 \mathrm{~ms}$ in qualitative agreement with the measurement presented in Fig. 35(b).

A second possible mechanism is the indirect coupling of nuclear spins due to the presence of a conduction electron in the QD (Abragam, 1961) as given by Eq.16. Such 

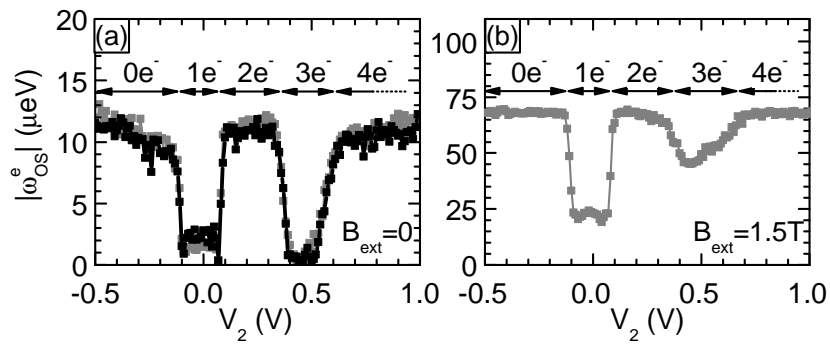

FIG. 36 (a) Nuclear spin polarization after free evolution of the nuclear spins during $\tau_{\text {wait }}=20 \mathrm{~ms}$ at a gate voltage $V_{2}$ for (a) $B_{e x t}=0$ and (b) $B_{\text {ext }}=1.5 \mathrm{~T}$. For odd numbers of QD electrons, DNP decays in a ms-timescale, while the decay takes seconds for an even number of electrons (gray (black) data points correspond to QD excitation with $\sigma^{+}$$\left(\sigma^{-}-\right)$polarized light. Adapted from Maletinsky (2008)

indirect interactions result in a long-range coupling of nuclear spins and can thereby significantly enhance nuclear spin diffusion rates. While this process conserves the total angular momentum of the nuclear spin system, it can lead to a decay of the Overhauser shift by re-distributing the nuclear spin polarization within the QD and by increasing the nuclear spin diffusion rate out of the QD. The resulting decay rate for the nuclear field has been estimated (Klauser et al. , 2006) to be on the order of $T_{\text {ind }}^{-1} \simeq \tilde{A}^{2} / \hbar^{2} N^{3 / 2} \omega_{Z}^{e}$, assuming a weakly polarized nuclear spin system and $\hbar \omega_{Z}^{e} \gg \tilde{A}$. It is important however that $T_{\text {ind }}$ gives the timescale over which coherent evolution due to the indirect interaction takes place and as such, only marks an upper limit to the nuclear spin relaxation rate due to indirect interactions (Klauser et al. 2006). An estimate of nuclear spin diffusion induced by indirect interactions is given in Sect.VI.C. Finally, indirect interaction would only lead to a partial relaxation of DNP, in contrast to the full decay shown in Fig. 34(b).

Electron-mediated nuclear spin relaxation is not limited to the regime where QDs are occupied by a single electron. Figure 36 shows a comparison of nuclear spin relaxation for QD charges between zero and four electrons. For this, the QD nuclei were first polarized at a QD gate voltage $V_{1}$ and then left to evolve for a fixed period of $\tau_{\text {wait }}=20 \mathrm{~ms}$ at a variable gate voltage $V_{2}$, before the final degree of DNP was measured with a short probe laser pulse. Figure 36 shows the resulting Overhauser shift as a function of the gate voltage $V_{2}$. Regions of fast and slow DNP decay can clearly be distinguished and correspond to QD occupations with an odd or even number of electrons, respectively ${ }^{12}$. When two electrons occupy the QD orbital ground state, the Pauli principle ensures that they form a spin singlet, which does not couple to the QD nuclei and therefore results in a prolonged lifetime

12 The QD charge state for a given gate voltage was independently identified through corresponding features in the QD PL spectra, as discussed by Urbaszek et al. (2003) of DNP on the order of seconds. The third QD electron occupies the next QD orbital (the $p$-shell), where it can again interact with the QD nuclei and lead to a fast depolarization of nuclear spins on a ms timescale. Injecting a fourth electron into the $\mathrm{QD}$ at $V_{g} \approx 0.6 \mathrm{~V}$ increases the DNP lifetime again, indicating that the two $p$-shell electrons form a spin-singlet state.

This last observation is at first surprising, since Hunds rule states that the first two electrons occupying the $p$-shell of a QD should form a spin-triplet at $B_{\text {ext }}=0$ (Warburton et al., 1998) and should therefore still couple to the QD nuclei. While the corresponding singlet-triplet splitting is typically on the order of $1 \mathrm{meV}$ (Karrai et al., 2004, Urbaszek et al., 2003), interactions breaking the rotational symmetry of the QD such as QD deformations (Schulhauser, 2004) or lattice effects (Bester et al. , 2007) can lower the singlet-triplet splitting and break Hunds rule, which explains the apparent singlet-character of the $p$-shell electrons in this QD. It is interesting to note that in this experiment, the QD nuclear spins can be seen as a probe for the properties of the QD and allow for an efficient test of Hunds rule in an individual QD.

To conclude this section, we would like to point out certain subtleties, originally discussed in Chapt. 11 of Dyakonov (2008), regarding the interpretation of the previously described measurements of DNP dynamics at low magnetic fields. For external magnetic fields smaller than the typical nuclear dipolar coupling (characterized by a dipolar field $B_{\mathrm{L}} \approx 0.1 \mathrm{mT}$ ), nuclear spin is in general not a conserved quantity, as dipolar interactions include "non-secular", spin non-conserving processes. Therefore, a nuclear polarization at zero magnetic field should completely relax within the nuclear coherence time $T_{\text {Dipole }} \propto 1 / B_{\mathrm{L}} \approx 100 \mu \mathrm{s}$. The long nuclear spin lifetimes shown in Fig. 35, seem to contradict this statement as DNP persists for a few minutes even at zero magnetic field (Maletinsky et al. 2007a). Two mechanisms could explain this discrepancy:

- If the relevant energy scale for QD nuclei is set by an interaction other than nuclear dipolar interaction, the above argument is invalid as non-secular dipolar coupling terms will be suppressed. This is the case if an external magnetic field is applied to the nuclei, but can also be induced at zero external field by quadrupolar interactions (see Sec.,V.B.2.

- As was already discussed in Sec.V.B.5, the Knight field of the electron can ensure nuclear spin polarization and thereby nuclear spin cooling, even at zero magnetic field ${ }^{13}$. In contrast to spin polarization, nuclear spin temperature is known to have

13 In the absence of any external magnetic field the natural spin quantization axis is normal to the QD plane, set by the stronger confinement along growth $(z)$ direction. The combination of circularly polarized excitation and the large spin-orbit interaction 


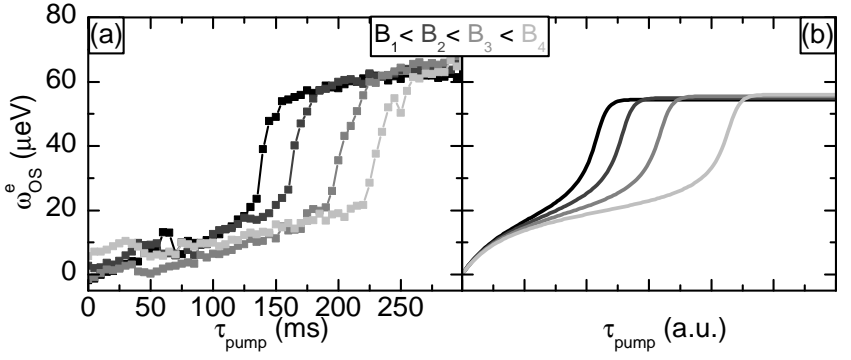

FIG. 37 (a) Buildup of DNP in external magnetic fields on the order of the final Overhauser field. Experiments were performed with the procedure and external parameters described in the main text at magnetic fields $B_{1}=1.1 \mathrm{~T}, B_{2}=1.2 \mathrm{~T}$, $B_{3}=1.3 \mathrm{~T}$ and $B_{4}=1.4 \mathrm{~T}$. (b) Simulations according to the classical nonlinear rate equation 19. The magnetic fields used for the simulation are $B_{1}=1.22 \mathrm{~T}, B_{2}=1.24 \mathrm{~T}$, $B_{3}=1.26 \mathrm{~T}$ and $B_{4}=1.28 \mathrm{~T}$. Adapted from Maletinsky (2008)

a slow relaxation time constant (Goldman, 1970) (characterized by $T_{1}$ ) and can therefore persist over timescales much longer than $T_{\text {Dipole }}$.

These two scenarios can be distinguished experimentally by changing the light-polarization of the probe pulse used in nuclear pump-probe experiments: In the case where nuclear spins are stabilized by quadrupolar interactions, the detected nuclear spin polarization should be independent of this polarization. Conversely, in the case of Knight-field stabilized nuclear spins, the sign of the nuclear spin polarization measured in the probe-pulse should follow the helicity of the probe laser-light. In particular, DNP should be zero for a linearly polarized probe pulse and parallel (antiparallel) to the initially created nuclear polarization if the probe pulse helicity is equal (opposite) to the pump pulse helicity.

These additional tests have been performed by Maletinsky (2008) and showed that in the case of self-assembled InAs QDs long nuclear spin lifetimes at zero magnetic field are indeed enabled by the strong nuclear quadrupolar shifts introduced in Sec. III.B ${ }^{14}$.

\section{B. Nonlinear nuclear spin dynamics in high magnetic fields}

In view of the nonlinear coupling between the electron and the nuclear spin system that was demonstrated

in the valence band then ensures that high-purity electron spin and heavy hole pseudo spin eigenstates can be prepared using laser pulses propagating along the $z$ direction. The average optically prepared electron spin is oriented parallel to the $z$ direction in this case.

14 We note that there is still a substantial degree of Knight-field stabilization in these systems as evidenced by the discussion in Sec.V.B.5 These Knight field related effects are likely to be caused by nuclei occupying spin states $m_{I}= \pm 1 / 2$. in Sec.V.B.1 the purely exponential buildup and decay curves presented in Sec.VI.A might come as a surprise. Since the nuclear spin relaxation rate $T_{1 \mathrm{e}}$ due to the QD electron depends on electron spin detuning, the buildup and decay rates of DNP should depend on the degree of nuclear spin polarization and therefore change during the time traces presented in Fig. 34. These nonlinear effects should be most prominent at the moment where the external and nuclear magnetic fields cancel and are therefore more easily observed at elevated external magnetic fields.

Nonlinearities in buildup and decay of DNP have been studied by Chekhovich et al. (2010b) and Maletinsky (2008) using pump-probe techniques similar to the ones described in the previous section. Figure 37(a) shows DNP buildup curves measured at various external magnetic fields and clearly demonstrates the discussed nonlinear buildup dynamics. A numerical simulation of the dynamics described by the nonlinear equation of motion Eq. (19) at the corresponding magnetic fields is presented in Fig. 37(b) and shows qualitative agreement with the experimental data.

A much more interesting situation arises for the decay of DNP in sizable external magnetic fields. Since the nuclear spin decay rate depends strongly on the electronic environment of the nuclei, the dependence of the electron-mediated DNP decay rate on $\omega_{Z}^{e}$ can have various forms, depending on the relative importance of the different possible mechanisms discussed in Sec.VI.A.1.

A good picture of the different decay characteristics at various $\mathrm{QD}$ gate voltages in high magnetic fields can be obtained by measuring DNP simultaneously as a function of gate voltage and time. The nuclei are first initialized in a state of maximally achievable DNP. The gate voltage is then switched to a value $V_{2}$ and DNP is measured after a waiting time $\tau_{\text {wait }}$. The measurement result as a function of $V_{2}$ and $\tau_{\text {wait }}$ is shown in Fig.38(a), where the final degree of DNP is encoded in gray-scale. In accordance with the discussion in Sec.VI.A.1, significant nuclear spin relaxation is only observed if the QD is occupied with a single electron. There, the decay rate shows a marked increase when $V_{2}$ approaches the edge of the $1 e^{-}$-plateau, where co-tunneling rates (introduced in section II.E increase substantially (Smith et al., 2005). This illustrates the importance of co-tunneling in electronmediated DNP decay, which is twofold: Co-tunneling ensures that the mean electron spin polarization is zero due to the coupling to the (unpolarized) electron reservoir and therefore sets the equilibrium nuclear spin polarization. Furthermore, co-tunneling shortens the electron spin correlation time $\tau_{c}^{e}$ which broadens the electron spin states and therefore allows for electron-nuclear spin flips to happen.

Some features in the data shown in Fig. 38 (a) seem to contradict the picture of electron-mediated nuclear spin decay. When approaching the $1 e^{-}-2 e^{-}$transition point $\left(0.02 \mathrm{~V}<V_{2}<0.04 \mathrm{~V}\right)$, nuclear spin lifetime increases again, even though the stable configuration of the QD 


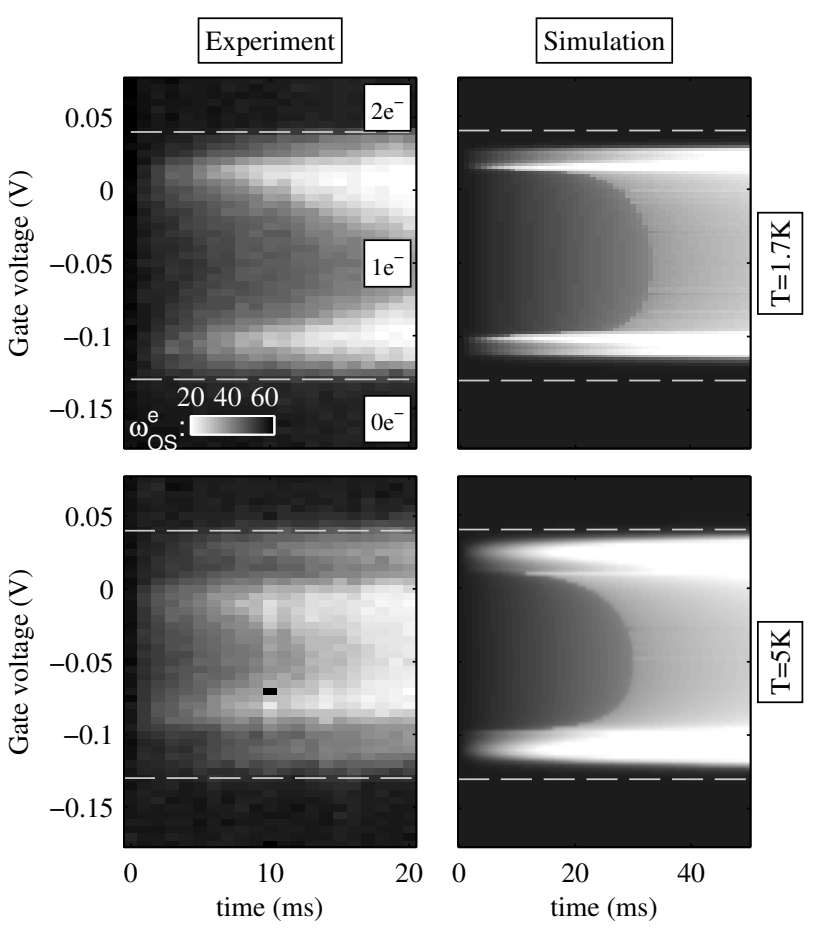

FIG. 38 Decay of DNP in an external magnetic field of $B_{\text {ext }}=1 \mathrm{~T}$. The nuclear spin polarization was initialized with a $100 \mathrm{~ms}, \sigma^{+}$-polarized pump pulse at a gate voltage $V_{1}$ corresponding to the center of the $1 e^{-}$-plateau, resulting in an initial Overhauser shift $\omega_{O S}^{e}\left(\tau_{\text {wait }}=0\right) \approx 55 \mu \mathrm{eV}$. Immediately after this nuclear spin initialization, the gate voltage was switched to a value $V_{2}$. (Left) Measurement of $\omega_{O S}^{e}$ in $\mu \mathrm{eV}$ as a function of waiting time $\tau_{\text {wait }}$ and gate voltage $V_{2}$ at $T=1.7 \mathrm{~K}$ (upper panel) and $T=5 \mathrm{~K}$ (lower panel). The dashed, gray lines indicate the transition between the QD charging states (Right) Simulations according to Eq. 18. Adapted from Maletinsky (2008)

is still singly charged. This observation is a signature of motional narrowing of the nuclear spins: While a finite $\tau_{c}^{e}$ is necessary to overcome the energy mismatch of the initial and final states of an electron-nuclear spin flip-flop, the nuclei cannot undergo such a transition if the electron spin fluctuations become too fast Abragam, 1961, Meier and Zakharchenya, 1984). This becomes apparent by inspecting Eq. (18) which shows that $T_{1 e}$ has a maximum for $\tau_{c}^{e}=1 / \omega^{e}$, after which $T_{1 e}^{-1}$ drops for decreasing $\tau_{c}^{e}$. We observe the maximal electron-nuclear spin relaxation rate at a gate voltage $V_{2}=0.02 \mathrm{~V}$. Since at $\tau_{\text {wait }}=0$ the total electron Zeeman splitting, $\hbar \omega^{e}$, amounts to $\sim 20 \mu \mathrm{eV}$, the corresponding electron cotunneling rate at this gate voltage should be on the order of $30 \mathrm{GHz}$, according to Eq. (18). This is in reasonable agreement with independent calculations of the electron co-tunneling rate in QD structures similar to the one studied here (Smith et al., 2005$)$. Motional narrowing is not observed on the $0 e^{-}-1 e^{-}$transition, where one would at first expect a similar behavior as in the $1 e^{-}-2 e^{-}$ transition. However, the electron tunneling rate is grow- ing with increasing gate voltage which leads to slower co-tunneling rates for the $0 e^{-}-1 e^{-}$transition compared to the $1 e^{-}-2 e^{-}$transition. $\tau_{c}^{e}$ might therefore never reach the value $1 / \omega^{e}$ on the low-voltage side of the $1 e^{-}$-plateau.

\section{Nuclear Spin dynamics at milliKelvin temperatures}

The experiments we described earlier in charge tunable InAs QDs have identified the role of exchange coupling to an electron gas in determining the nuclear spin relaxation in single-electron charged QDs. It is then natural to ask if elimination of the ensuing co-tunneling processes by either increasing the tunnel barrier thickness or by reducing the temperature would reveal other, intrinsic Overhauser field decay mechanisms ${ }^{15}$. Motivated by this question, Latta et al. (2011b) have studied the Overhauser field dynamics on a $35 \mathrm{~nm}$ tunnel barrier sample (compared to the more conventional $25 \mathrm{~nm}$ studied in the previous section) in a regime where the coupling to the degenerate electron gas is vanishingly small and the Overhauser field dynamics is determined solely by the coupling of each nucleus to a confined electron spin. These experiments revealed that in an external magnetic field of $\mathrm{B}_{z}=5 \mathrm{~T}$ in Faraday geometry and temperatures of $\sim 200 \mathrm{mK}$ there are two distinct mechanisms for the Overhauser field decay:

(1) a spatially limited, temperature-independent, nuclear spin diffusion within the dot originating from electron mediated nuclear spin interactions

(2) a co-tunneling mediated, temperature dependent (Dreiser et al., 2008 ), decay of the Overhauser field approaching $\tau_{\text {decay }} \approx 10^{5}$ s. Remarkably, the diffusion induced reduction in the Overhauser field taking place on $\sim 100 \mathrm{~s}$ timescale can be strongly suppressed by repeating the preparation cycle consisting of polarization (pump) and free-evolution (wait). In these experiments an Overhauser field was established by resonant dragging of the blue-shifted Zeeman line of the $X^{0}$ transition, as described in Sec.V.C.3 followed by a waiting period $\tau_{\text {wait }}$ at a gate voltage where the $\mathrm{QD}$ contained a single electron and the laser field is completely off-resonant with all QD transitions. Finally the Overhauser field remaining after $\tau_{\text {wait }}$ was determined by first ejecting the resident electron from the QD and then scanning the laser field across the $X^{0}$ resonance quickly so as to measure but not destroy the QD nuclear polarization.

When the gate voltage is chosen such that the QD is singly charged with a co-tunneling rate $\left(\kappa \geq 10^{7} s^{-1}\right)$ that does not allow for appreciable electron spin pumping (Atature et al. 2006), the Overhauser field exhibits a fast decay (Latta et al., 2009, Maletinsky et al., 2007b) on

\footnotetext{
15 The optical investigation of nuclear spin dynamics is carried out almost exclusively in the regime where $k T>\omega_{Z}^{e}$ (for the singular exception of this section VI.C. In this regime, the spin relaxation and excitation rates are both proportional to $\mathrm{kT}$.
} 
the order of a few seconds. The observed decay is temperature dependent, consistent with the predictions of the co-tunneling mediated process discussed in Sect.VI.A.1.

When the exchange coupling between the QD electron and the Fermi reservoir is minimized by choosing a gate voltage during $\tau_{\text {wait }}$ corresponding to the center for the $X^{-}$plateau, the temperature dependence of the long-time decay rate becomes even more prominent: Figure 39 (a) shows two measurements at $4 \mathrm{~K}$ and $200 \mathrm{mK}$ of the Overhauser field magnitude as a function of waiting time $\tau_{\text {wait }}$. In both cases, there is an initial partial decay taking place at $\sim 100 \mathrm{~s}$ time-scale which saturates after the Overhauser field decays to half of its value. The slow decay at $4 \mathrm{~K}$ takes place on a time scale of $5 \times 10^{3} \mathrm{~s}$, whereas at $200 \mathrm{mK}$ the corresponding decay time exceeds $10^{4} \mathrm{~s}$.

The temperature-dependent decay of the Overhauser field can be explained by a second order process originating from an effective non-collinear dipolar hyperfine interaction $\hat{H}_{\mathrm{hf}}^{\mathrm{nc}}$ of Eq.15. as discussed in Sec. V.C.3 such an effective coupling appears when the quadrupolar axes $z^{\prime}$ of the QD nuclear spins are not parallel to the external field $B_{z}$. The energy conservation in this irreversible nuclear spin flip process is ensured by the exchange coupling of the QD electron to the degenerate electron gas leading to a co-tunneling rate $\kappa=1 / \tau_{\text {cot }}$; the corresponding Overhauser field decay rate can then be shown to be $\left(A_{\mathrm{nc}}^{i} / \hbar \omega_{Z}^{n}\right)^{2} / \tau_{\text {cot }}$. In the studied sample $\tau_{\text {cot }}$ is estimated to be on the order of $10 \mathrm{~ms}$ at $4 \mathrm{~K}$ in the plateau center which allows to obtain the value of $A_{\mathrm{nc}}^{i} \simeq 0.03 \tilde{A}_{i}$ for the effective non-collinear dipolar hyperfine coupling constant between the electron and the nuclei. It should be emphasized that the energy difference of the states coupled by $\hat{H}_{\mathrm{hf}}^{\mathrm{nc}}$ is given by $\omega_{Z}^{n}<10 \mathrm{mK}$ which is in turn much smaller than the electron temperatures that can be reached. This observation suggests that the relevant co-tunneling rate and hence the Overhauser field decay rate will simply be linearly proportional to the electron temperature. Finally, even though $1 / \tau_{\text {cot }} \ll \omega_{Z}^{n}$, the fact that $T \gg \omega_{Z}^{n}$ ensures the validity of the Markov approximation in describing the nuclear spin flips associated with $\hat{H}_{\mathrm{hf}}^{\mathrm{nc}}$.

As is seen in Fig. 39(a), the observed nuclear spin dynamics is much richer than a simple exponential decay curve. To explain the initial partial decay of DNP taking place on $\mathrm{a} \sim 100 \mathrm{~s}$ time scale, we consider the nuclear spin spatial diffusion mediated by an indirect interaction through the electron present in the dot already introduced in Sec.V.A.

$$
\hat{H}_{\text {ind }}=\sum_{i, j} \frac{A^{i} A^{j}}{\omega_{Z}^{e}} \hat{S}_{z} \hat{I}_{+}^{i} \hat{I}_{-}^{j}
$$

This Hamiltonian ensures the conservation of the total nuclear spin polarization and leads to diffusion within the region where the electron wave-function is non-vanishing. Although the total magnitude of QD nuclear spin polarization does not decrease due to this diffusion process, the
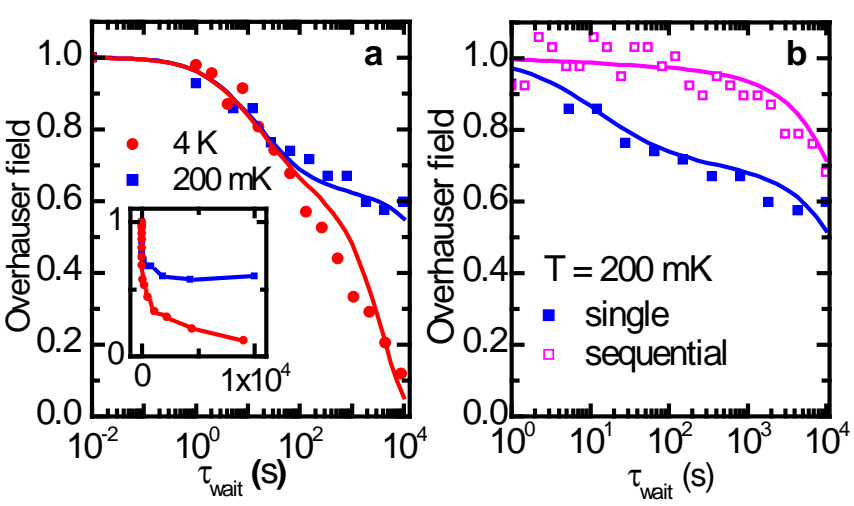

FIG. 39 a) Decay of the Overhauser field with negligible cotunneling for the case of a resident electron $\left(V_{\text {wait }}=530 \mathrm{mV}\right)$ at $200 \mathrm{mK}(\boldsymbol{\square})$ and $4 \mathrm{~K}(\bullet)$. The inset shows the same data in a linear-linear plot. b) Demonstration of the spatially limited nuclear spin diffusion: By sequential polarization of the nuclear spins, the polarization can be saturated, suppressing further nuclear spin diffusion (Latta et al. 2011b)

Overhauser field seen by the electron decays partially due to a redistribution of the nuclear spin polarization within the QD.

A strong evidence that the initial partial decay of the experienced Overhauser field stems from $\hat{H}_{\text {ind }}$ is provided by repeating the polarization-wait-measure cycle and observing its effect on the decay dynamics. In these experiments, it was ensured that the initial value of the Overhauser field (immediately after the polarization cycle) is identical for all repetitions. The total waiting time in each cycle was chosen to be $200 \mathrm{~s}$, which is longer than the time scale over which the initial limited decay takes place. The corresponding decay of the Overhauser field is shown in Fig. 39(b). Clearly, the initial limited decay is suppressed in this case, indicating a saturation of the nuclear spin polarization within the QD. The experimental data are in excellent agreement with numerical calculations based on semi-classical rate equations taking into account electron mediated diffusion and the pure decay of nuclear spin polarization (Latta et al., 2011b). The model assumes that the coherent evolution described by $\hat{H}_{\text {ind }}$ is interrupted by a dephasing process; the absence of temperature dependence of the saturable decay and hence the dephasing process suggests that the relevant noise is channelled through electrical wiring. Finally, the finite bandwidth of the measurement set-up ensured that this electrical noise has vanishing contribution at $\omega_{Z}^{n}$, explaining why there is no temperature-independent contribution to the decay processes stemming from $\hat{H}_{\mathrm{hf}}^{\mathrm{nc}}$.

\section{Dynamic nuclear polarization in a transverse field: the anomalous Hanle effect}

Since a finite nuclear polarization can be achieved in InAs/GaAs QDs along the optical axis $z$ in zero external magnetic field (see Sec.V.B.5), it is obviously interesting 
to study its evolution against a transverse magnetic field $\boldsymbol{B}=B_{x} \boldsymbol{u}_{x}$. In particular, the quadrupolar interaction with principal axis $\| z$ is expected to inhibit the alignment of the nuclear polarization parallel or anti-parallel to $\boldsymbol{B}$, as it could be expected from Eq. (17), up to fields of the order of a few $B_{\mathrm{Q}}$ 's. The splitting $n \times \hbar \omega_{\mathrm{Q}}$ between the states $|n \pm 1 / 2\rangle$ reduces indeed drastically the effect of the magnetic coupling, except for the states $| \pm 1 / 2\rangle$ $(n=0)$ which still split linearly in low field according to $\Delta_{ \pm 1 / 2}=2 \hbar \gamma_{n} B_{x}$. Their spin polarization $\| z$ should thus be cancelled in a very small transverse field. In contrast, the pairs of levels $| \pm m\rangle$ with $|m|>1 / 2$ have a vanishingly small splitting $\propto \hbar \gamma_{n} B_{x}\left(B_{x} / B_{\mathrm{Q}}\right)^{2 m-1}$ as long as $B_{x}<B_{\mathrm{Q}}$. One may thus infer that their nuclear polarization should be substantially preserved in fields below $B_{\mathrm{Q}}$, maintaining a nuclear field $\| z$ of a few $100 \mathrm{mT}$ 's.

Interestingly, the evolution of the nuclear field can be investigated through the decrease of electron spin polarization in the transverse field $B_{x}$ (Hanle effect) which is directly accessible from the photoluminescence of $X^{+}$ trion photo-created with a circularly polarized excitation. In the absence of nuclear effects, such Hanle depolarization curves assume a Lorentzian profile with half width $B_{1 / 2}=\hbar /\left(\left|g_{e}\right| \mu_{B} \tau_{r}\right)$. Deviations from this well understood behavior are usually caused by nuclear effects and can thus be used to study DNP in QDs. Such measurements of the Hanle effect have been very successful in the past to demonstrate nuclear spin cooling in the electron Knight field at very low external fields for bulk semiconductor, as well as the magnetic anisotropy of nuclei due to the quadrupolar interaction in alloys like AlGaAs (Meier and Zakharchenya, 1984). More recently, the Hanle effect has been measured in an ensemble of p-type doped InP/InGaP QDs (Dzhioev and Korenev, 2007): the halfwidth $B_{1 / 2}$ of the Lorentzian decrease was found to be 3 times larger when a nuclear field was created (under constant $\sigma^{+}$excitation) than in absence of nuclear polarization (excitation with $50 \mathrm{kHz}$ modulated $\sigma^{+} / \sigma^{-}$polarization). From this observation it was concluded that a finite nuclear field is maintained perpendicular to the external field thanks to the quadrupolar splitting of the levels as described above. However, a direct measurement of the Overhauser field to confirm this hypothesis is not possible in experiments on ensembles of QDs.

The study of individual InAs/GaAs QDs allows to measure both the Hanle effect and the Overhauser field to further refine this interpretation, while revealing more pronounced anomalies of the Hanle depolarization curves.

Hanle effect in single QDs has been first investigated in interfacial GaAs QDs (Bracker et al. 2005 ), yet in conditions where no nuclear field was generated. Besides, for these unstrained and almost pure GaAs QDs the electric field gradient should cancel out on nuclear sites making the quadrupolar interaction vanishingly small. Depolarization curves following a Lorentzian profile were indeed obtained by Bracker et al. with a $B_{1 / 2}$ field in good agreement with the expected estimate. In con-
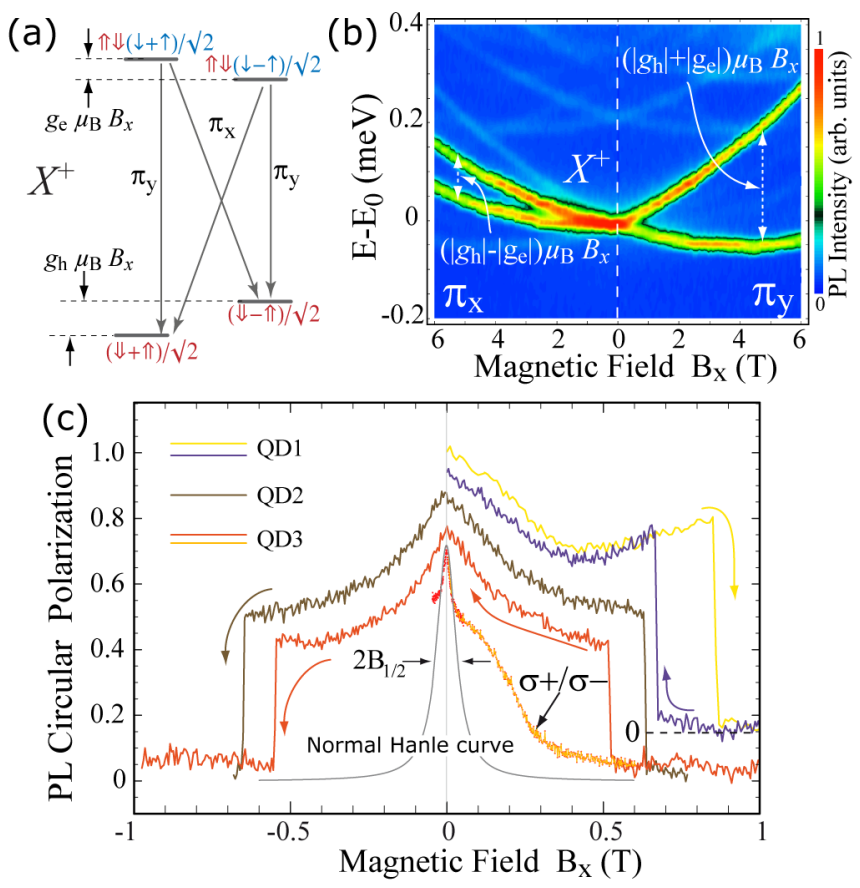

FIG. 40 (a) Schematics of optical transitions and polarization rules for an $X^{+}$trion in a transverse magnetic field $B_{x}$. (b) Typical density plot of $X^{+}$PL intensity from a single QD against magnetic field $B_{x}$ and detection energy around $E_{0}=1.355 \mathrm{eV}$. The excitation is linearly polarized and the detection either $\pi_{x}$ or $\pi_{y}$ as indicated. (c) Depolarization curves (Hanle effect) of $X^{+}$lines from 3 different QDs under $\sigma^{+}$quasi-resonant excitation. QD1 measurements are vertically shifted for clarity. (after Krebs et al. (2010))

trast, a strongly anomalous Hanle effect is reported for InGaAs/GaAs QDs under excitation conditions producing DNP in zero field (Krebs et al., 2010). For these QDs the theoretical $B_{1 / 2}$ field (in absence of nuclear polarization) amounts to $\sim 30 \mathrm{mT}$. Note that the value of $g_{e}$ which determines $B_{1 / 2}$ can be precisely inferred from the Zeeman effect in a magnetic field $B_{x} \gtrsim 1 \mathrm{~T}$, since it produces 4 distinct linearly polarized transitions with splittings given by $\left(\left|g_{e}\right| \pm\left|g_{h}\right|\right) \mu_{\mathrm{B}} B_{x}$, see Fig.40(a),(b). As shown in Fig. 40(c), the experimental Hanle curves of $X^{+}$trions reveal that a sizeable electron spin polarization $\sim 50 \%$ is maintained in fields as high as $\sim 1 \mathrm{~T}$, up to a critical field $B_{x}^{c}$ where it abruptly collapses. Moreover, this evolution is symmetrical in magnetic field which means that it does not depend on the specific helicity $\sigma^{+}$or $\sigma^{-}$ of the illumination while keeping the field direction constant. However, under excitation with $50 \mathrm{kHz}$-modulated $\sigma^{+} / \sigma^{-}$polarization such that no nuclear field $\| z$ is created, the electron spin stabilization is significantly reduced.

These observations agree qualitatively well with the interpretation that a nuclear field $\| z$ would be maintained thanks to the quadrupolar interaction. Yet, the magnitude of this nuclear field should be as high as $B_{x}^{c}$ in order to keep the electron spin polarization above $50 \%$ up to 
$B_{x}^{c}$. The direct measurement of this nuclear field component $B_{n, z}$, deduced as in Faraday configuration from the splitting of the $\sigma^{ \pm}$lines, indicates in contrast that $B_{n, z}$ monotonically decreases with $B_{x}$ from a maximum value around $0.35 \mathrm{~T}$, down to $\sim 0 \mathrm{~T}$ at $0.5 \mathrm{~T}$ (Krebs et al. 2010), which obviously invalidates the above interpretation. If a nuclear field is responsible for the electron spin stabilization, it must be nearly opposite to the external field $\left(B_{n, x} \approx-B_{x}\right)$, such that the in-plane component of the total field remains smaller than $B_{1 / 2}$ up to $B_{x}^{c}$.

Determining the actual magnetic field experienced by the electron in the field range of interest, namely around $B_{x}^{c}$ requires in principle a very high spectral resolution to separate the four different lines of $X^{+}$. However, if the hole $g$-factor is sufficiently large, this requirement can be circumvented because the splittings of the $\pi_{x}$-polarized lines and $\pi_{y}$-polarized lines can be measured separately for fields as small as $\sim 0.5 \mathrm{~T}$, with a $\pi_{x^{-}}$or $\pi_{y}$-polarized detection while keeping a $\sigma^{+}$excitation, see Fig. 41(a). For both polarizations the $X^{+}$splitting turns out to be reduced solely to the hole Zeeman splitting $g_{h} \mu_{\mathrm{B}} B_{x}$ for fields below $B_{x}^{c}$ and present jumps at $B_{x}^{c}$ where they recover their normal values $\left(g_{h} \pm g_{e}\right) \mu_{\mathrm{B}} B_{x}$, see Fig. 41.(c). This behavior proves that the anomalous Hanle effect is due to a nuclear field which essentially cancels out the applied field, as depicted in Fig.41(b). The detailed mechanism leading to the establishment of this in-plane nuclear field is yet to be elucidated, the possible role of the noncollinear hyperfine interaction (see Sec.V.C.3 deserves further investigation. In this context, we remark that a mean field approach such as the one proposed in Dzhioev and Korenev (2007) predicts in contrast the build-up of an in-plane nuclear field pointing in the same direction as the transverse field.

\section{E. Optically detected NMR on semiconductor quantum dots}

The Overhauser shift of the emission spectrum of a single dot allows for a direct measurement of the steady state nuclear spin polarization. Further information about the QD composition and the QD nuclear spin system can in principle be gained through optically detected nuclear magnetic resonance (NMR) experiments. Under optical pumping conditions, the spin polarization of the nuclei can be decreased by applying an oscillating magnetic field orthogonal to the nuclear spin quantization axis at a frequency which matches the nuclear level splitting in the RF range. This drives transitions between nuclear spin states and the corresponding change in nuclear spin polarization can be detected via changes of the Overhauser shift. The first such experiment has been demonstrated by Gammon et al. (1997) in GaAs interface fluctuation QDs and has provided the first direct proof of nuclear spin pumping in these QD systems. Figure 42 (a) shows the observed NMR spectra of ${ }^{75} \mathrm{As}$ and ${ }^{69} \mathrm{Ga}$; the corresponding resonance lines are remarkably
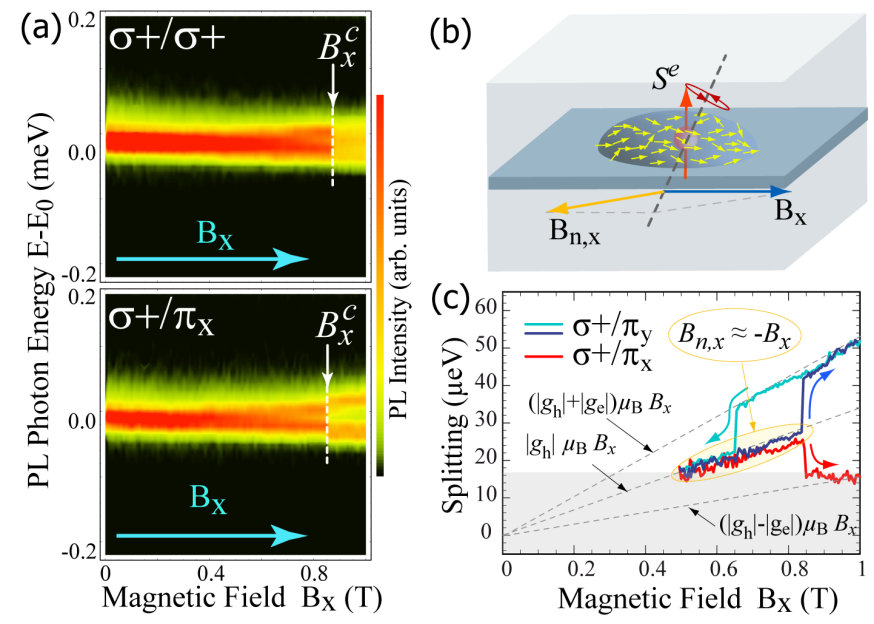

FIG. 41 (a) Comparison of $X^{+}$PL density plots measured under $\sigma^{+} / \sigma^{+}$or $\sigma^{+} / \pi_{x}$ configurations of excitation/detection. polarizations. The latter enables one to resolve the transition splittings for fields above $\sim 0.5 \mathrm{~T}$ and thus to infer the nuclear field. (b) Sketch of the nuclear polarization generated almost parallel to $B_{x}$ giving rise to a nuclear field $B_{n, x} \approx-B_{x}$ responsible for the anomalous Hanle effect. (c) Splitting of $X^{+} \pi_{x^{-}}$and $\pi_{y}$-polarized transitions evidencing the cancellation of the external field for $B_{x}<B_{x}^{c}$. Normal splittings are recovered above $B_{x}^{c}$ when the nuclear polarization is eventually destroyed. (adapted from Krebs et al. (2010) )

narrow, indicating that the nuclear spin system in interfacial QDs is rather unperturbed by inhomogeneous strain or other line-broadening mechanisms linked to nuclear quardrupole effects (see Sec.III.B ${ }^{16}$. Another linebroadening mechanism can stem from (inhomogeneous) Knight-shifts of NMR lines which are caused by the effective magnetic field that a spin-polarized electron exerts on the QD nuclei (see Sec.III.A). NMR line-broadening and line-shifts due to this Knight-field were recently observed by Makhonin et al. (2010) in NMR experiments in individual interfacial QDs.

Performing optically detected NMR experiments on the well isolated nuclear spin system in self assembled QDs, such as InAs QDs in GaAs, has remained an open challenge for many years. Only very recently first optically detected NMR experiments on large ensembles of self assembled InAs QDs were reported by Cherbunin et al. (2011); Flisinski et al. (2010). The difficulty in observing NMR in self-assembled QDs lies in the significant inhomogeneity of the nuclear spin system due to the strongly strained lattice of these QDs. Strain in the QD lattice results in strong, inhomogeneous quadrupolar splittings of the nuclear spin states. As a result, NMR

\footnotetext{
16 Gammon et al. (1997) note however a certain variability of NMR frequencies between different QDs as well as occasional lines which are anomalously broad.
} 
lines become strongly broadened and are difficult to observe.

Flisinski et al. (2010) partly circumvent these difficulties in two ways: The splitting between nuclear $m_{s}=$ $\pm 1 / 2$-states is invariant under nuclear quadrupole interactions (Abragam, 1961) and the corresponding NMR lines are thus expected to be insensitive to inhomogeneous quadrupolar fields. Alternatively, by sweeping the NMR driving frequency over a broad range, all possible nuclear spin transitions can be addressed simultaneously. Flisinski et al. (2010) employ both these techniques to detect NMR in Hanle depolarization experiments performed on a large ensemble of self-assembled InGaAs QDs. Fig.42(b) shows modifications of Hanle curves due to the presence of a RF field which depolarizes the nuclei. This data clearly shows that nuclear spins in self-assembled QDs can be addressed using RF driving fields together with optical detection of DNP. By keeping the frequency of the NMR driving field fixed, Flisinski et al. (2010) were able to observe resonant NMR features in the low-field region of the Hanle depolarization curves (Fig. 42(b), inset). These resonances correspond to transitions between $m_{I}= \pm 1 / 2$ states of ${ }^{71} \mathrm{Ga}$ and ${ }^{75} \mathrm{As}$ and provide an additional fingerprint of the strong quadrupolar interactions present in the QDs studied in this work: For 3/2-spins (such as ${ }^{71} \mathrm{Ga}$ and ${ }^{75} \mathrm{As}$ ) the gyromagnetic ratio for the $m_{I}= \pm 1 / 2$ is enhanced by a factor of two in the presence of strong quadrupolar interactions and a magnetic field applied perpendicular to the quantization axis of the spins. The data shown in Fig.42(c) confirms this picture and demonstrates that the strain in the QDs under study is predominantly oriented along the QD growth direction. The broadening of the NMR lines in Fig. 42(b) can then be interpreted as a variation of strainaxis, within individual QDs or between different QDs in the ensemble.

Finally we note that recent advances in optically detected NMR spectroscopy in single dots have allowed an isotope sensitive determination of hyperfine constants for holes coupled to nuclear spins in both strained and unstrained dot systems (Chekhovich et al. 2011).

\section{F. Irreversibility and hysteresis in demagnetization experiments}

A remarkable feature of the nuclear-spin system of InAs/GaAs QD is the excellent isolation from its environment if the QD is charged with an even number of electrons and kept in the dark (see Sec.VI.A and VI.B. In the specific case where all charge carriers are removed from the QD after a sequence of optical DNP, the relaxation of the nuclear polarization turns out to be extremely slow. Its characteristic time is shown to exceed one hour when the QD is subject to a longitudinal magnetic field $B_{\text {ext }}$ in the $0-2$ T range, see Fig. 35 and Maletinsky et al. (2009). This clearly indicates that not only the dipolar relaxation but also the nuclear spin (a) GaAs / AIGaAs dot
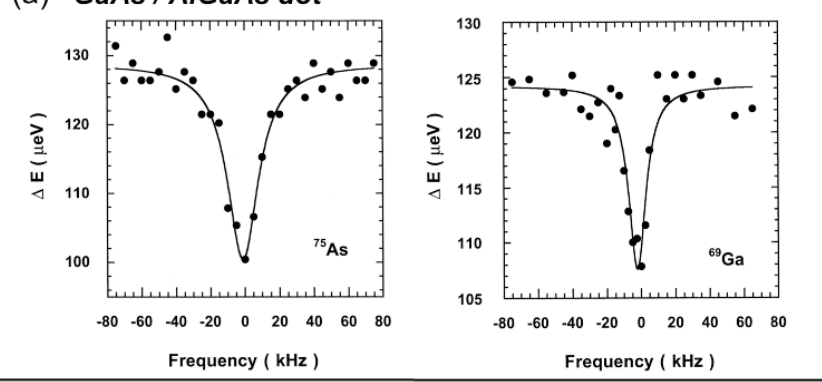

InGaAs / GaAs dots
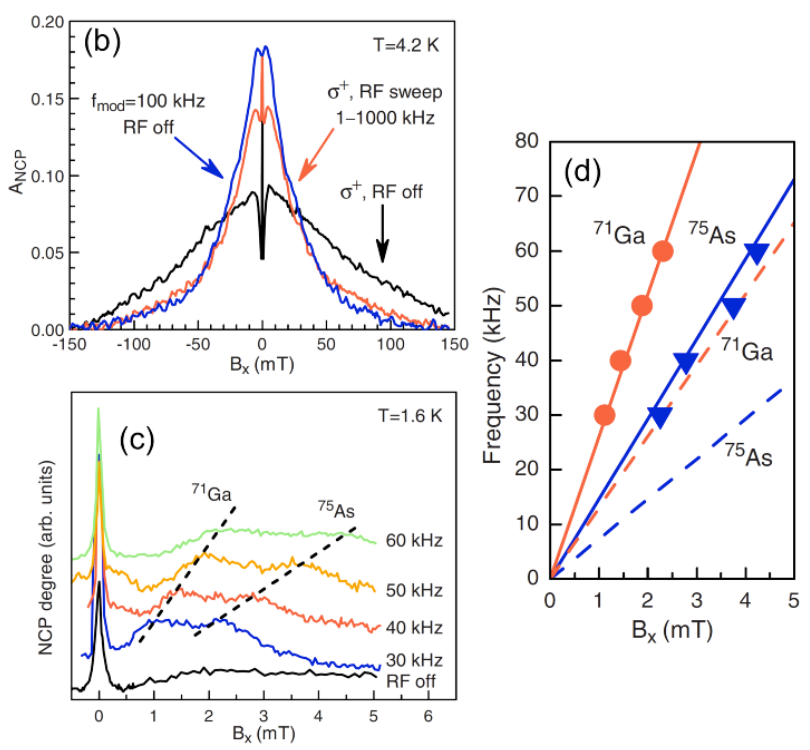

FIG. 42 Optically detected nuclear magnetic resonance in semiconductor QDs (a) NMR spectra of a single interfacial, GaAs QD: The Zeeman splitting of the QD in a longitudinal magnetic field of $1 \mathrm{~T}$ changes as a function of the frequency of an applied transverse RF magnetic field when the frequency matches the nuclear Larmor precession frequency (adapted from Brown et al. (1998)). Frequency offset for ${ }^{75} \mathrm{As}$ : $7.274 \mathrm{MHz}$, for ${ }^{69} \mathrm{Ga}: 10.193 \mathrm{MHz}$. (b) Hanle curves measured on an ensemble of self-assembled InAs QDs for $\sigma^{+}$-polarized excitation in absence and presence of an RF field applied along the z-axis. The radio-frequency is scanned over all the In and As nuclear magnetic resonances for the relevant magnetic field range. For comparison, the Hanle curve detected for excitation with polarization modulation at $f_{\text {mod }}=100 \mathrm{kHz}$ is shown. (c) Effect of fixed radio-frequency irradiation (with $\mathrm{RF}$ field along the $\mathrm{y}$ axis), which reveals distinct nuclear resonances for the low-field range indicated by the dashed rectangle (curves are shifted vertically for clarity). (d) Magnetic field dependencies of resonant frequencies extracted from the inset in (a). The dependencies of NMR for $m_{I}= \pm 1 / 2$ states in ${ }^{71} \mathrm{Ga}$ and ${ }^{75} \mathrm{As}$ nuclei calculated with and without quadrupole interaction are shown by the solid and dashed lines, respectively (adapted from Flisinski et al. (2010)). 
diffusion towards the surrounding material are strongly suppressed. Under this circumstance, manipulating the nuclear polarization by slowly varying an external parameter like the magnetic field is technically possible. Besides its intrinsic interest it may also provide valuable information on the thermodynamics of the nuclear-spin system in one QD.

While the nuclear polarization achieved under constant circularly polarized optical excitation depends drastically on the magnetic field (see Sec.V.B.3), if the QD is isolated, under conditions where relaxation is suppressed, its polarization should evolve adiabatically as a function of a slowly varying magnetic field. In the absence of quadrupolar interaction, the nuclear spin component $\| z$ is a good quantum number so that the change of the external field should not produce any change of the nuclear level populations and the initial nuclear polarization should remain constant. Only in magnetic fields $\lesssim B_{L}$, the local field induced by the dipolar interaction between nuclei, should the polarization decrease and eventually vanish in strictly zero field ${ }^{17}$. Yet, if the system is well isolated (no heat flow) its entropy does not change during the adiabatic demagnetization, so that applying again a magnetic field above $B_{L}$ restores in principle the initial polarization. This thermodynamic approach corresponds to a description of the system by a spin temperature $T_{\mathrm{s}}$ which is related to the nuclear polarization according to Curie's law. Since the entropy of the QD spin ensemble depends only on the ratio $\left(B_{\text {ext }}^{2}+B_{\mathrm{L}}^{2}\right) / T_{\mathrm{s}}^{2}$ (Slichter, 1990), the adiabatic demagnetization leads to a reduction (in absolute value) of $T_{\mathrm{s}}$ proportional to the field until $B_{\text {ext }} \approx B_{\mathrm{L}}$. Remarkably, when a magnetic field above $B_{\mathrm{L}}$ is re-applied Curie's law predicts that the polarization should be recovered and aligned parallel to the field, independent of its orientation, in the same or opposite direction depending only on the sign of $T_{\mathrm{s}}$. Such reorientation of an initially created nuclear polarization $\| z$ to a transverse direction was nicely evidenced in bulk GaAs (Meier and Zakharchenya, 1984), demonstrating convincingly the validity of the spin temperature concept in this case. Clearly, in a self-assembled QD with large quadrupolar interaction the validity of the spin temperature concept requires further consideration.

To address this issue in the case of InAs/GaAs QDs, adiabatic demagnetization experiments have been performed in a longitudinal magnetic field (Maletinsky et al. 2009). The procedure is sketched in Fig.43(a). The nuclear spins of a single QD are first polarized by optical pumping for a duration $\tau_{\text {pump }} \approx 600 \mathrm{~ms}$ and in an initial field $B_{\mathrm{i}}=2 \mathrm{~T}$ parallel to $z$. When the excitation is switched off, the gate voltage of the sample is immedi-

\footnotetext{
17 Note that for an empty dot (neither conduction electron nor valence hole present) the depolarization due to the indirect interaction of Eq. 16 as used to describe nuclear spin depolarization in fields of $5 \mathrm{~T}$ in Sec.VI.C is not applicable as it requires the presence of charge carriers.
}

ately changed to uncharge the dot (to assure long nuclear spin relaxation times, as in Fig. (35), and the magnetic field is slowly varied at a rate $\gamma_{\mathrm{B}}=10 \mathrm{mT} \mathrm{s}^{-1}$ to a final value $B_{\mathrm{f}}$. To read out the remaining polarization, an electron is re-injected in the dot by applying the required voltage and a linearly-polarized optical pulse, short enough to not destroy the DNP, is used to measure the Overhauser shift from the $X^{-}$trion PL. The normalized polarization $P_{\text {nuc }}\left(B_{\mathrm{f}}\right) / P_{\text {nuc }}\left(B_{\mathrm{i}}\right)$ following the demagnetization process $B_{\mathrm{i}} \rightarrow B_{\mathrm{f}}$ is plotted in Fig. 43 (b) with $B_{\mathrm{f}}$ going from $2 \mathrm{~T}\left(B_{\mathrm{f}}=B_{\mathrm{i}}\right)$ to $-1 \mathrm{~T}$. These measurements are completed by re-magnetization experiments where the field is ramped down and up according to $B_{\mathrm{i}} \rightarrow B_{\mathrm{f}}^{\prime} \rightarrow B_{\mathrm{f}}$ with $B_{\mathrm{f}}^{\prime}=-1 \mathrm{~T}$, in order to probe the reversibility of the whole process. At first glance, the system obeys satisfactorily the Curie's law: the nuclear polarization is almost constant when the field decreases from $B_{\mathrm{i}}$ down to $\sim 0.3 \mathrm{~T}$, then decreases rapidly and changes sign when the magnetic field passes through zero, and eventually recover to a constant level for $B_{\mathrm{f}}<-0.3$ T. Significant discrepancies to the spin temperature model are yet noticeable: there is a finite remnant nuclear polarization $P_{\text {nuc }}^{\text {rem }} \simeq 0.2$ at $B_{\mathrm{f}}=0 \mathrm{~T}$, the field at which the polarization starts decreasing or recovering is much higher than the dipolar field $B_{\mathrm{L}}$, and the final polarization recovered in negative fields amounts to only $60 \%$ of its initial magnitude. When the QD is remagnetized towards positive field a similar behavior is observed so that a complete cycle evidences a drastic irreversibility. Figure 43(d) shows that the irreversibility for a round trip field ramp $B_{\mathrm{i}} \rightarrow B_{\mathrm{f}}^{\prime} \rightarrow B_{\mathrm{i}}$ develops for $\left|B_{\mathrm{f}}^{\prime}\right|<0.3 \mathrm{~T}$.

The strain-induced quadrupolar interaction of selfassembled QDs is most certainly responsible for the observed disagreements to the spin temperature description. On the one hand, the inhomogeneous dispersion of the principal axis angle $\theta$ produces anti-crossings of the nuclear levels in fields of a few $100 \mathrm{mT}$, see Fig.19(a,b). When the field ramp passes through anti-crossings of energy splitting $\hbar \omega_{\mathrm{S}} \approx \hbar \theta \omega_{\mathrm{Q}}$ between states with $\Delta m= \pm 1$ and such that $\omega_{\mathrm{S}} \gg \sqrt{\gamma_{n} \gamma_{\mathrm{B}}} \sim 300 \mathrm{~Hz}$ (Landau-Zener criterion for adiabatic anti-crossing), the population of each level is conserved while the corresponding eigenstates are progressively exchanged. One ends up with an effective exchange of the actual populations and therefore an inversion of their relative polarization, e.g. $p_{+3 / 2} \rightleftarrows p_{+1 / 2}$ for $B_{z}$ around $B_{\mathrm{Q}}$ when the $|+3 / 2\rangle$ and $|+1 / 2\rangle$ states anticross. This explains why the polarization starts decreasing in fields around $0.3 \mathrm{~T}$. On the other hand, the quadrupolar splitting quenches the effect of the dipolar interaction in zero field for states $|m|>1 / 2$. The typical dipolar splitting of $| \pm 1 / 2\rangle$ states $\hbar \gamma_{n} B_{\mathrm{L}}$ is reduced by factors $\left(B_{\mathrm{L}} / B_{\mathrm{Q}}\right)^{2 m-1} \ll 1$ for those states, so that the randomization of their polarization is suppressed yielding a finite $P_{\text {nuc }}^{\text {rem }}$ in zero field. The latter is noticeably larger than that directly created in zero field (see Sec.V.B.5), but obviously decreases with the magnitude of the initial polarization when the initial field $B_{\mathrm{i}}$ 
decreases (Fig.43(c)). Actually the pairs of levels $| \pm m\rangle$ with $|m|>1 / 2$ do cross in zero field in the sense that their splitting $\ll \sqrt{\gamma_{n} \gamma_{\mathrm{B}}}$. Therefore and in contrast to the $| \pm 1 / 2\rangle$ states, their polarization is conserved without changing sign when the field passes through zero. (a)

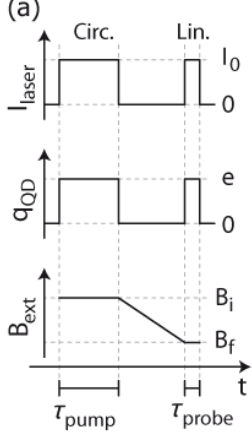

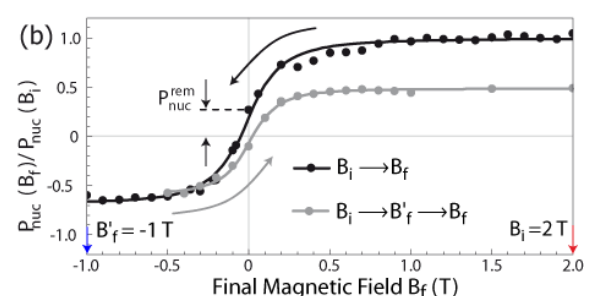

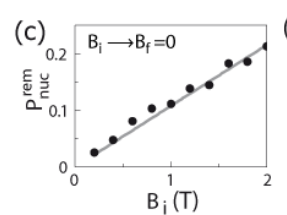

(d)

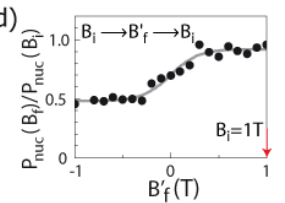

FIG. 43 (a). Schematic diagram of the experimental procedure for adiabatic demagnetization of QD nuclear spins in an charge tuneable InGaAs/GaAs QD. The nuclei are optically pumped at $B_{\text {ext }}=B_{\text {i }}$ via quasi-resonant excitation of $X^{-}$ trion while the $\mathrm{QD}$ is in charge state $q_{\mathrm{QD}}=1 e$. Immediately after the pumping pulse, the electron is ejected from the QD. $B_{\text {ext }}$ is then linearly ramped at a rate $\gamma_{\mathrm{B}}$ to a final value $B_{\mathrm{f}}$, at which the nuclear polarization $P_{\text {nuc }}$ is measured with a short linearly polarized optical pulse. (b) Demagnetization as a function of the final magnetic field $B_{\mathrm{f}}$, for a monotonic decrease from $B_{\mathrm{i}}=2 \mathrm{~T}$ (dark) and for a return from an intermediate field $B_{\mathrm{f}}^{\prime}=-1 \mathrm{~T}$ (gray). (c) Remanent polarization at zero field normalized to the polarization generated at $2 \mathrm{~T}$, when $B_{\mathrm{i}}$ is varied. (d) Nuclear polarization following a complete round trip as indicated. (adapted from Maletinsky et al. (2009))

The combination of these polarization-conserving crossings and polarization-inverting anti-crossings is mostly responsible for the reduction of the polarization which is recovered in fields $<-0.3 \mathrm{~T}$. However, the pronounced irreversibility observed when the QD is remagnetized can not be explained by these processes which are perfectly reversible. A specific mechanism leading to the increase of the system entropy is required to explain this observation. In Maletinsky et al. (2009), it has been suggested that cross-relaxation between pairs of nuclear spins should be dramatically enhanced at specific fields where a harmonic configuration of any three nuclear levels is created (Abragam, 1961). Efficient thermal relaxation involving a coupling to the environment (in particular to the unpolarized nuclei surrounding the QD) would then make these crossover transitions irreversible. This interpretation was shown to agree well with a numerical simulation of the demagnetization and re-magnetization curves relying on the adiabatic evolution of the populations as discussed above, but including this irreversible process (Maletinsky et al. 2009).

In addition, one could question if all 2-level crossings or anti-crossings of a given nucleus are truly adiabatic.
Assuming an average dipolar local field $B_{L} \sim 0.15 \mathrm{mT}$, the zero field splitting of $| \pm m\rangle$ states, $\gamma_{n} B_{\mathrm{L}} \sim 1 \mathrm{kHz}$, is roughly of the same order as $\sqrt{\gamma_{n} \gamma_{\mathrm{B}}}$ at the ramping rate $\gamma_{\mathrm{B}}$ of $10 \mathrm{mT} \mathrm{s}^{-1}$. As a result, the adiabaticity condition is probably not verified for all the nuclei in a magnetic field ramp through zero. Similarly, for Indium nuclei $(I=9 / 2)$ the quadrupolar interaction gives rise to many different anti-crossings at different magnetic fields; their typical splittings cover several orders of magnitude because for small $\theta$ they scale as $\hbar \omega_{Q} \theta^{|\Delta m|}$ where $|\Delta m| \in\{1, \ldots, 8\}$ is the difference of angular momentum. Taking into account in addition the dispersion of $\theta$ in a $\mathrm{QD}$, it seems very likely that for a large fraction of nuclei in a QD there is at least one pair of anti-crossing levels such that $\omega_{\mathrm{Q}} \theta^{|\Delta m|} \sim \sqrt{\gamma_{n} \gamma_{\mathrm{B}}}$, which would result in irreversibility. The fact that the observed irreversibility (see Fig. 43(d)) develops essentially in the field region where these quadrupole-induced anti-crossings take place, rather supports the above idea, although we note that in these experiments changes in the magnetic field ramp speed did not cause any noticeable effect on the experimental results.

\section{HOLE SPINS COUPLED TO NUCLEAR SPINS}

The interaction between valence hole spins and nuclear spins has usually been ignored in semiconductors for two main reasons. First, the $p$-symmetry of the periodic part of the valence Bloch wave function results in negligible overlap with the nuclear spins yielding vanishing Fermi contact interaction. Therefore $\hat{H}_{h f}^{f c}$ of Eq 3 , that is at the origin of the hyperfine interaction discussed for conduction electrons in sections IV, V and VI does not apply. Second, the hole spin in bulk or quantum well structures is very fragile: the hole spin relaxation time is of the order of 10 ps or less in bulk GaAs due to strong heavy-light hole mixing in the valence bands (Meier and Zakharchenya, 1984), which leads to a correlation time for the hyperfine interaction that is too short to achieve a significant dynamic nuclear polarization. In QDs the hole spin is much more robust than in bulk or quantum well structures because of the discrete energy states (Flissikowski et al., 2003, Heiss et al., 2007; Laurent et al., 2005) and significant effects linked to the hole-nuclear spin interaction have been revealed.

The hyperfine interaction of nuclear spins with an electron in the valence band is primarily dipolar. For a given nucleus, the Hamiltonian of this interaction reads (Abragam, 1961):

$$
\hat{H}_{\mathrm{hf}}^{\mathrm{dip}}=2 \mu_{B} \frac{\mu_{I}}{I} \hat{\boldsymbol{I}} \cdot\left[\frac{\hat{\boldsymbol{L}}}{\rho^{3}}-\frac{\hat{\boldsymbol{S}}}{\rho^{3}}+3 \frac{\boldsymbol{\rho}(\hat{\boldsymbol{S}} \cdot \boldsymbol{\rho})}{\rho^{5}}\right]
$$

where $\mu_{B}$ is the Bohr magneton and $\mu_{I}$ is the nuclear magnetic moment; $\hat{I}$ is the nuclear spin operator; $\boldsymbol{\rho}$ is the electron position vector with respect to the nucleus; $\hat{L}$ and $\hat{S}$ are the electron orbital momentum and spin 
operators, respectively. The dipolar hyperfine interaction depends on the valence band mixing $\beta$, which quantifies the deviation of the confined hole from the ideal, pure heavy hole case. In a simple picture, for a heavy hole $J_{z}=3 / 2$ to do a flip-flop with a nucleus $\left(\Delta J_{z}=-1\right)$, it would have to access the $J_{z}=1 / 2$ light hole state. This is energetically forbidden as heavy and light holes are separated in energy by $\Delta_{H L}$. Valence band mixing is thus required to circumvent this blockade. The valence band mixing $\beta$ may arise from anisotropic strain fields within the QD and/or shape and interface anisotropy (Belhadj et al., 2010, Krebs and Voisin, 1996: Krizhanovskii et al. 2005). This makes it necessary to consider the mixed hole states by including the light hole component as in:

$$
|\widetilde{ \pm 3 / 2}\rangle=\frac{1}{\sqrt{1+|\beta|^{2}}}(| \pm 3 / 2\rangle+\beta|\mp 1 / 2\rangle)
$$

where $|\beta|$ should be much smaller than unity, as a direct consequence of the energy splitting $\Delta_{H L}$ between heavy and light hole states (tens of meV in InAs/GaAs dots). The dipolar hole spin-nuclear spin hamiltonian can then be written as:

$\hat{H}_{\mathrm{hf}}^{\mathrm{dip}}=\nu_{0} \sum_{j} \frac{A_{j}^{h}}{1+\beta^{2}}\left|\psi\left(\boldsymbol{r}_{j}\right)\right|^{2}\left(\hat{I}_{z}^{j} \hat{S}_{z}^{h}+\frac{\alpha}{2}\left[\hat{I}_{+}^{j} \hat{S}_{-}^{h}+\hat{I}_{-}^{j} \hat{S}_{+}^{h}\right]\right)$

where $\alpha=\frac{2|\beta|}{\sqrt{3}}$ is the anisotropy factor Fischer et al. 2008, Testelin et al., 2009). In the case of moderate heavy-hole light-hole mixing and assuming a constant wavefunction $\psi(\boldsymbol{r})=\sqrt{2 / N \nu_{0}}$ an approximate, simplified hamiltonian is obtained:

$$
\hat{H}_{\mathrm{hf}}^{\mathrm{dip}}=\frac{2 \tilde{A}^{h}}{N}\left(\hat{I}_{z} \hat{S}_{z}^{h}+\alpha\left[\frac{\hat{I}_{+} \hat{S}_{-}^{h}+\hat{I}_{-} \hat{S}_{+}^{h}}{2}\right]\right) .
$$

Here $\tilde{A}^{h}$ is the average value of the dipole-dipole hyperfine constant (Gryncharova and Perel, 1977; Testelin et al. 2009), $\hat{S}_{ \pm}^{h}$ denote the heavy hole pseudo-spin $1 / 2$ operators acting on $\left|\frac{\tilde{3}}{2}\right\rangle$.

The Hamiltonians $\hat{H}_{\mathrm{hf}}^{\mathrm{fc}}$ for electrons and $\hat{H}_{\mathrm{hf}}^{\mathrm{dip}}$ for holes have a similar form (compare Eq. (6) and Eq. (34)), but there are important differences:

(i) the ratio $\left|\tilde{A}^{h}\right| / \tilde{A}$ is about $\simeq 0.1$, as has been theoretically predicted by (Fischer et al. 2008$)$ followed by experimental demostrations as in Fig.444(c) and Fig.445 Chekhovich et al. (2011a); Eble et al. (2009); Fallahi et al. (2010); Testelin et al. (2009);

(ii) the amplitude of the flip-flop term is proportional to heavy-hole light-hole mixing, which varies from dot to dot.

In the framework of the simple quantum box model, the strength of the flip-flop process $\tilde{A}$ for electrons directly depends on the materials that form the $\mathrm{QD}$, whereas the dipolar flip-flop term for holes not only depends on the chemical composition of the dot, but also on the valence band mixing $\beta$, which takes into account (a)

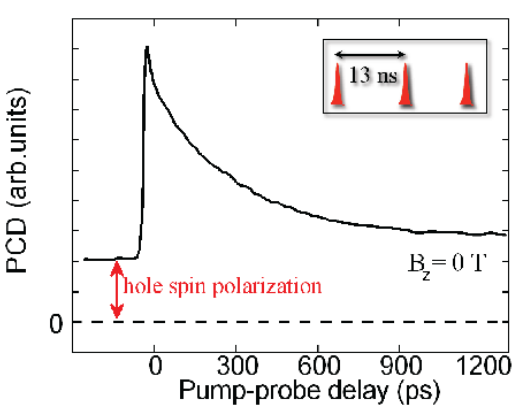

(b)

(c)
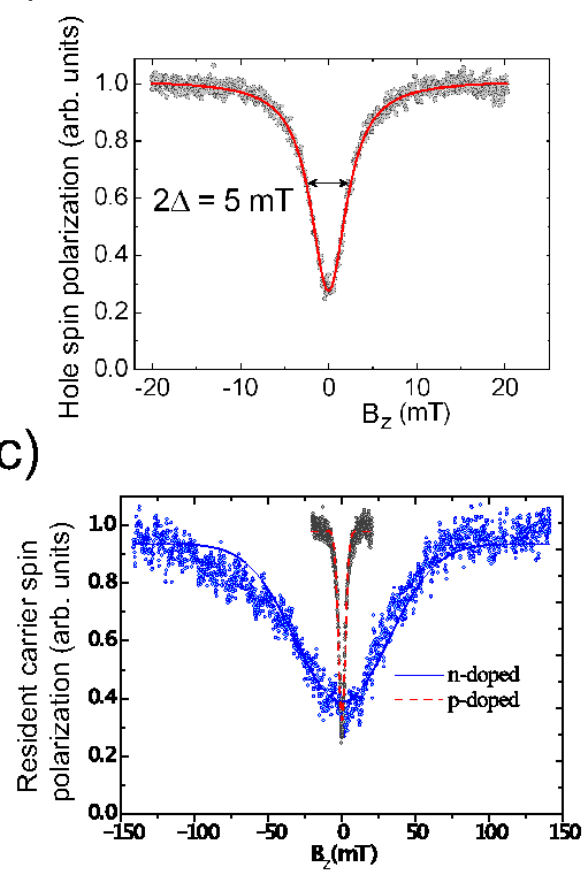

FIG. 44 (a) photo-induced circular dichroism (PCD) signal as a function of pump-probe delay, when the p-doped QDs are excited by a periodic train of ultra-short pulses. (b) normalized PCD amplitude at negative pump-probe delay $t=-130$ ps (i.e. reflecting the hole spin polarization) versus the applied longitudinal magnetic field $B_{z}$, performed on a $\mathrm{QD}$ sample with one hole per dot. The HWHM equals $2.5 \mathrm{mT}$. The solid line is a fit using the model developed in Eble et al. (2010) . (c) blue curve: normalized PCD amplitude at negative pump-probe delay $t=-130 \mathrm{ps}$ (i.e. reflecting the electron spin polarization) versus the applied longitudinal magnetic field, performed on QDs charged with one electron. The HWHM equals $47 \mathrm{mT}$. Similar measurement on QDs charged with one hole (red curve) is added on the same figure to directly compare the efficiency of hyperfine induced dephasing for electrons and holes (Desfonds et al. 2010).

fine details of the real QD spatial strain distribution and shape anisotropy. In recent measurements, $\tilde{A}^{h}$ was found to be negative for In and $\mathrm{Ga}$, and positive for As in different dot systems made out of III-V compounds (Chekhovich et al., 2011), the origin of this surprising finding is still under discussion. 


\section{A. Hole spin dephasing due to hyperfine interaction}

As the hyperfine interaction with the fluctuating nuclear field is severely limiting electron spin coherence, the hole spin with negligible Fermi contact hyperfine interaction seems a more promising candidate for achieving long spin coherence times. With this motivation in mind, detailed investigations led to promising reports (Brunner et al., 2009, Gerardot et al., 2008) under certain experimental conditions. On the other hand, surprisingly short hole spin dephasing times due to nuclear spin fluctuations were also reported (Eble et al. 2009). To clarify the limitations for hole spin coherence, an the of hole spin dynamics in the model system of singly positively charged InAs QDs is discussed below, revealing that dipole-dipole hyperfine interaction does play an important role in decoherence of the resident hole spin at cryogenic temperatures.

Figure 44(a) shows the photoinduced circular dichroism (PCD) signals versus pump-probe delay, obtained when no magnetic field is applied in an ensemble of $\mathrm{p}$ doped QDs containing a resident hole. The experiments are performed under modulated $\sigma^{+} / \sigma^{-}$helicity of the excitation laser pulse $\left(f_{\bmod }=50 \mathrm{KHz}\right)$ in order to avoid any dynamic nuclear spin polarization (here $\left\langle\hat{I}_{z}\right\rangle=0$ ). A nonzero PCD signal at negative pump-probe delays is observed, indicating that the spin polarization is not fully relaxed within the $T_{L}=13 \mathrm{~ns}$ repetition period of the laser pulses. This long-living component of the PCD signal is directly associated with the net spin polarization of the resident holes, the only species present in the sample after the radiative recombination of $X^{+}$trions $\left(\tau_{r} \sim 800 \mathrm{ps}\right)$. From this experimental observation, one can understand how the spin polarization of the resident holes in the dot is built-up by a resonant pulsed excitation, in the following sequential way : (i) the $\sigma^{+}$circularly polarized pump beam photo-creates spin oriented trions $X^{+}$with electron spin $\downarrow$. (ii) During the lifetime of the excited states, the efficient electron-nuclear hyperfine interaction leads to a coherent coupling of their spin projections along the light direction. (iii) Finally, the spontaneous decay of the trion states by emission of polarized photons leads to an unbalanced hole-spin population with $\rho_{\Uparrow}^{h}>\rho_{\Downarrow}^{h}$ (as in the hole spin pumping scheme of Fig. 17(a)).

Figure 44(b) shows the experimental data for the PCD signal at negative delays, $\operatorname{PCD}\left(0^{-}\right)$, i.e. the hole-spin polarization at $13 \mathrm{~ns}$, as a function of the applied magnetic field. The application of a small magnetic field $B_{z}$ in the milli-Tesla range has a dramatic impact on the hole spin polarization. The Lorentzian -like dependence with a half width at half maximum (HWHM) of only $2.5 \mathrm{mT}$ is interpreted as the progressive magnetic field quenching of the hyperfine-induced hole spin relaxation, just as in the case of the electron (Fig.9(a)), but at much lower field. For $\mathrm{B}=0$ the hole spin dephasing time due to the interaction with nuclear spins is of the order of $10 \mathrm{~ns}$ (Eble et al., 2009).
To give a first interpretation that only involves hyperfine-induced dephasing it is convenient to treat, $\hat{H}_{\mathrm{hf}}^{\mathrm{fc}}$ and $\hat{H}_{\mathrm{hf}}^{\mathrm{dip}}$ from Eq. (34) (see Merkulov et al. (2002) and Testelin et al. (2009)), as semi-classical magnetic fields randomly distributed from dot to dot. The orientation of the nuclear field fluctuations $\delta B_{n}$ responsible for electron spin dephasing is isotropic. In stark contrast, $\delta B_{n}^{h}$ is highly anisotropic and the corresponding Gaussian distribution of the nuclear field $B_{n}^{h}$ acting on holes at zero average nuclear field is:

$$
W\left(B_{n}^{h}\right) \propto \exp \left(-\frac{\left(B_{n, z}^{h}\right)^{2}}{2\left(\delta B_{n, \|}^{h}\right)^{2}}\right) \exp \left(-\frac{\left(B_{n, x}^{h}\right)^{2}+\left(B_{n, y}^{h}\right)^{2}}{2\left(\delta B_{n, \perp}^{h}\right)^{2}}\right)
$$

where $\delta B_{n, \perp}^{h}=\alpha \delta B_{n, \|}^{h}$ and $\delta B_{n, \perp}^{h}=\hbar /\left(g_{h}^{z} \mu_{B} T_{\Delta}^{h}\right)$, directions $\perp$ and $\|$ with respect to the $z$-axis (i.e. quantization and light propagation axis). $T_{\Delta}^{h}$ is the ensemble spin dephasing time, arising from the random hole precession directions and frequencies in the randomly distributed frozen nuclear field. Following the same approach as Merkulov et al. (2002), adapted for the anisotropic interaction of hole spins with nuclei by Testelin et al. (2009), the decay of the z-component of the hole pseudo-spin is given by ${ }^{18}$ :

$$
T_{\Delta}^{h}=\hbar \frac{1+\beta^{2}}{2 \beta / \sqrt{3}}\left(\frac{3 N}{4 \sum_{\varepsilon} I^{\varepsilon}\left(I^{\varepsilon}+1\right)\left(A_{j}^{h}\right)^{2}}\right)^{1 / 2}
$$

where $\varepsilon=$ In, As or Ga. Using a heavy-light hole mixing characterized by $|\beta|=0.4$, this equation yields a hole spin dephasing time of the order of $10 \mathrm{~ns}$, comparable to the one which has been measured in the PCD experiment. As the electron -nuclear spin dephasing time can be measured in the same p-doped sample by photoluminescence dynamics (see Fig. $9(\mathrm{a})$ ), the ratio between the electron and hole nuclear spin dephasing time can be extracted: it is found for these dots that the dephasing time of the hole with nuclear spins is about ten times longer than the dephasing time of the electron spins, but it is not negligible. Note that the exact values of $|\beta|$ are extremely dot and sample dependent. The heavy hole - light hole mixing depends on the exact dot shape and strain which can both vary significantly from dot to dot, even in the same sample. $|\beta|=0.4$ used to fit experiments as in Fig. 44(b) corresponds to a very strong mixing in the sample investigated in Eble et al. (2009), whereas for dots of the same material used in other studies (Fallahi et al., 2010, Gerardot et al., 2008) the mixing deduced from analysing optical pumping experiments is significantly smaller.

PCD measurements also allow revisiting the study performed on electron spin dynamics by PL experiments.

\footnotetext{
18 Note that in our simple approach this time diverges for $\beta \rightarrow 0$, but the dephasing time is still finite in this limit, see theory of Fischer et al. (2008) and recent experiments (Fras et al. 2011).
} 
Figure 44(c) presents the PCD evolution versus longitudinal magnetic field performed on an n-doped QD ensemble. The interaction between nuclei and the electron takes place in the ground state of the QDs after $X^{-}$recombination. The external magnetic field can efficiently cancel the effect of the hyperfine interaction on the carrier-spin dephasing time if its magnitude becomes larger than the corresponding effective nuclear field fluctuation. This field is of the order of several tens of $\mathrm{mT}$ for electrons (see Fig.10 and Fig.11 of section IV.C), as can be seen in the comparison with the values for holes shown in Fig. 44(c) (Desfonds et al., 2010): the relative strength of the hole to electron hyperfine coupling deduced from these measurements is on the order of $10 \%$. In summary, the hole spin confined to a QD does interact with the fluctuating nuclear spins through dipole-dipole coupling. The strength of this interaction depends on the degree of heavy hole-light hole mixing and is a significant source of decoherence.

There are still open questions concerning the exact link between valence band mixing and the transverse components of the hyperfine interaction: A strong in-plane hyperfine field for holes and the resulting short hole spin coherence time is a priori linked to strong heavy hole-light hole mixing (Testelin et al. 2009). Conversely, measuring strong heavy hole-light hole mixing by analysing the transverse hole g-factor, for example, does not automatically result in short hole spin decoherence times for the QDs investigated (Brunner et al., 2009). This underlines that hole spin dephasing due to the hyperfine interaction is a very recent research topic (Eble et al. 2009; Fischer et al. 2008), that will surely stimulate further innovative theoretical and experimental investigations (Godden et al. 2012, Greilich et al., 2011, Greve et al., 2011).

\section{B. Overhauser effect for holes}

In addition to a spin dephasing contribution, the nonzero interaction between hole and nuclear spins can also lead to the observation of Overhauser effect for holes. An existing nuclear polarization can split pure heavy hole spin states (Overhauser effect) due to the Ising term $\frac{2 \tilde{A}^{h}}{N} \hat{I}_{z} \hat{S}_{z}^{h}$ in Eq. (34) (see Chekhovich et al. (2011a); Fallahi et al. (2010); Fischer et al. (2008)).

The dynamic nuclear polarization created through non-resonant excitation of an InP dot was monitored by Chekhovich et al. 2011a via the emission of the neutral exciton bright states $|\Uparrow \downarrow\rangle$ and $|\Downarrow \uparrow\rangle$ and the dark states $|\Uparrow \uparrow\rangle$ and $|\Downarrow \downarrow\rangle$. Here, the dark exciton states are weakly optically active due to heavy hole - light hole mixing. It is therefore possible to compare for the same dot the effect of a finite nuclear polarization on (i) the electron spin by measuring the energy difference between the $|\Downarrow \uparrow\rangle$ and $|\Downarrow \downarrow\rangle$ state and (ii) very interestingly, the hole spin by measuring the energy difference between the $|\Uparrow \uparrow\rangle$ and $|\Downarrow \uparrow\rangle$. Figure 45 shows directly, that the hyperfine constant in the investigated InP QDs is about a factor of 10

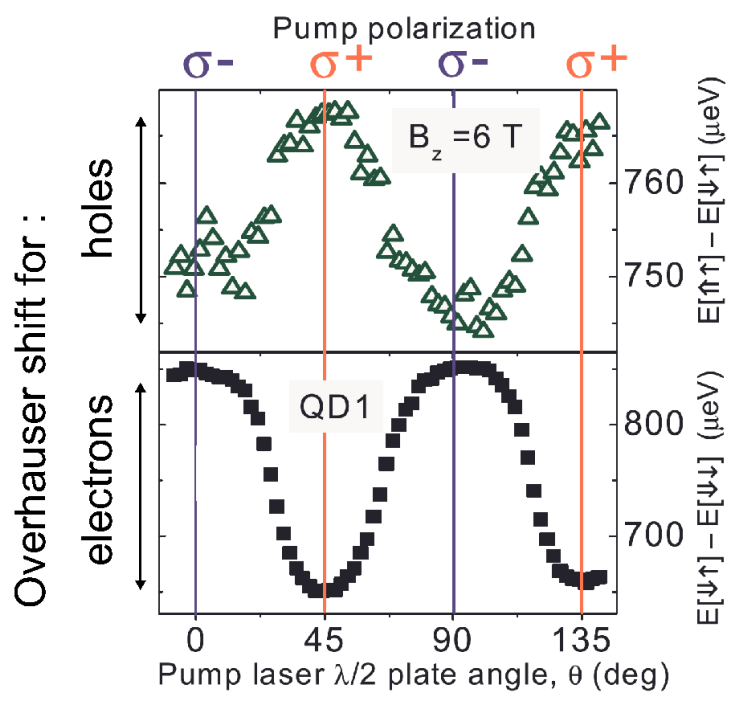

FIG. 45 Measurement of the electron- and hole nuclear spin interaction in a neutral $\mathrm{InP} \operatorname{dot}$ at $B_{z}=6 \mathrm{~T}$. The angle of a $\lambda / 2$ plate is varied to change the polarization of the pump laser resulting in a change of nuclear spin polarization (Chekhovich et al. 2011a).

stronger for electrons than for holes, and the signs are opposite (note the different vertical scales). The relative strength of the hyperfine interactions as well as the relative sign of the electron and hole Overhauser effect can vary for different nuclear spin species (Chekhovich et al. 2011). In InAs dots the strength and the sign of hyperfine interaction of the heavy hole with nuclear spins has also been measured (Fallahi et al. 2010$)$ ). By using an experiment based on the locking of the QD resonance to the incident laser frequency (see Sec.V.C.3), it has been possible to measure very accurately the Overhauser shift due to electron or exciton (including both electron and hole contributions). A ratio $\simeq-0.1$ of the heavyhole and electron hyperfine interaction has been deduced a value very similar to the one measured in InP dots (Chekhovich et al. 2011a). In both of these experiments the hole feels the nuclear field which has been dynamically created. The relative contribution of electron and hole spins to DNP is an open issue (Xu et al. 2009, Yang and Sham, 2010), as in most optical experiments a single spin of either species is present in the dot at some stage of the absorption-emission cycle. As $\tilde{A} \gg \tilde{A}^{h}$, DNP due to the electron will most likely dominate when electrons are present during some stage in the dot.

\section{PERSPECTIVES}

The physics reviewed in this article has progressed immensely over the past years due to the fruitful exchange of ideas from distinct scientific communities working on nuclear magnetism, electron spin physics in nanostructures, quantum optics and quantum dot photonics. For 
the latter, experiments without applied magnetic fields are important for potential applications. Here for example the impact of the nuclear spin bath on the carrier spin polarization and hence emitted photon polarization via the optical selection rules has recently been shown to play an important role in the context of entangled photon pair emission (Stevenson et al. 2011 ). Even at $B_{z}=0$ the screening of the nuclear field fluctuations $\delta B_{n}$ by an optically created Overhauser field $B_{n}$ has been shown to allow for tuning of the polarization states of both charged (Lai et al., 2006) and neutral excitons (Belhadj et al., 2009). Here the exact interplay between the Knight field $B_{K}$ and quadrupolar effects due to strain and alloy disorder that allows the creation of nuclear polarization is yet to be clarified. A promising route will be the investigation of strain free systems such as GaAs/AlGas droplet dots for which quadrupolar effects are far less important. This latter system has the additional advantage of containing dots truly isolated from each other as no wetting layer is formed under certain growth conditions (Sallen et al. 2011) permitting studies of nuclear spin diffusion across the AlGaAs barrier (Malinowski et al., 2001) and comparison with the physics of GaAs dots formed due to interface fluctuations in GaAs/AlGaAs quantum wells (Gammon et al. 2001).

One challenge is to prolong carrier spin dephasing times in quantum dots by eliminating the effects of the fluctuating nuclear field $\delta B_{n}$ without changing its mean value. Here sophisticated spin echo techniques in both quantum dot optics (Press et al., 2010) and transport (Bluhm et al. 2010$)$ are promising routes to decouple the electron spin from the nuclear spin bath. In electron spin resonance experiments again the interplay between electron spin dephasing and dynamic nuclear polarization can be expected to give rise to surprising, non-linear behavior and memory effects (Kroner et al., 2008).

An alternative approach is to try to polarize as many nuclear spins as possible to finally achieve a net reduction in the nuclear spin fluctuations $\delta B_{n}$ once the nuclear polarization is approaching $100 \%$. So far the maximum nuclear polarization achieved in different material systems is about $60 \%$ (Bracker et al. 2005 , Chekhovich et al., 2010a; Urbaszek et al. 2007). Future experiments should clarify if this nuclear polarization presents some fundamental limit as dark nuclear states might form (Imamoglu et al. , 2003) or if complete nuclear spin polarization is accessible in experiments in dots where each lattice nucleus has non-zero nuclear spin (Feng et al. 2007). In the regime of complete nuclear spin polarization, the possibility of using the long lived, well isolated nuclear spin system as a quantum memory (Taylor et al. 2003 Witzel and Das Sarma, 2007)) can be explored.

Another exciting approach is based on the recent observation of the locking of the QD resonance to an optical (Latta et al., 2009, Xu et al. 2009) or microwave driving (Vink et al. |2009) field (see Sec.|V.C). Here the achieved dynamical polarization is far below $100 \%$ but the amplitude of the nuclear field fluctuations $\delta B_{n}$ can be reduced significantly under certain conditions. Further reduction of the nuclear field fluctuations are the motivation of further experimental and theoretical work (Högele et al. 2012, Issler et al. 2010; Yang and Sham, 2010) that aims to clarify the exact nature of the interplay between Fermicontact and dipole-dipole type interactions for electrons and holes with nuclear spins.

The mesoscopic nuclear spin system of one QD coupled to a single electron spin may be used to study exciting cooperative effects such as phase transitions (Kessler et al. 2012, 2010). The reason for the abrupt collapse of the nuclear polarization experienced by an electron in a transverse magnetic field shown in Fig.40 is not understood (Krebs et al., 2010$)$ and one could speculate that collective phenomena play a role. If this is realistic for quantum dots with strong quadrupolar effects has to be clarified, ideally trying to do similar experiments in transverse fields in dots with vanishing quadrupolar effects (Belhadj et al. 2008, Gammon et al. 2001).

The emission of polarized photons as a result of the radiative recombination of electrons with well defined spin and (unpolarized) holes is at the heart of an important type of device called Spin-LEDs, often with QDs in the active region (Asshoff et al., 2011a, Li et al., 2005, Lombez et al. 2007b). The carriers are injected electrically, the electrons spin orientation is assured by the passage through a ferromagnetic contact. Due to the robustness of the electron spin even this highly non-resonant carrier injection leads to substantial electron spin polarization in the QD ground state and first promising results show that this carrier polarization leads in turn to measurable Overhauser fields (Asshoff et al. 2011b), paving an alternative way for electrical control of nuclear spin polarization.

The fascinating physics of one carrier spin coupled to a mesoscopic nuclear spin ensemble has been revealed through optical investigations of semiconductor QDs. Very sophisticated transport measurements on gate defined QDs have provided an alternative approach to study the coupled spin systems, resulting in an impressive level of control of spin coherence and relaxation (for example Bluhm et al. (2010); Hanson et al. (2007); Petta et al. (2005); Takahashi et al. (2011)). A promising approach for the future might be to combine fast and convenient optical techniques to manipulate spins in high quality gate defined QDs.

\section{Acknowledgments}

We would like to thank ANR QUAMOS, ITN SPINOPTRONICS and NCCR Nanoscience for support. We thank Mete Atature, Manfred Bayer, Benoit Eble, Khaled Karrai, Martin Kroner, Chih-Wei Lai, Alexander Tartakovksii and Richard Warburton for many fruitful discussions. 


\section{References}

Abragam, A., 1961, Oxford University Press .

Akimov, I. A., D. H. Feng, and F. Henneberger, 2006, Phys. Rev. Lett. 97(5), 056602.

Akimov, I. A., A. Hundt, T. Flissikowski, and F. Henneberger, 2002, Applied Physics Letters 81(25), 4730.

Akopian, N., N. H. Lindner, E. Poem, Y. Berlatzky, J. Avron, D. Gershoni, B. D. Gerardot, and P. M. Petroff, 2006, Phys. Rev. Lett. 96(13), 130501.

Alen, B., A. Hogele, M. Kroner, S. Seidl, K. Karrai, R. J. Warburton, A. Badolato, G. Medeiros-Ribeiro, and P. M. Petroff, 2006, Applied Physics Letters 89(12), 123124.

Amasha, S., K. MacLean, I. P. Radu, D. M. Zumbühl, M. A. Kastner, M. P. Hanson, and A. C. Gossard, 2008, Phys. Rev. Lett. 100, 046803.

Asshoff, P., A. Merz, H. Kalt, and M. Hetterich, 2011a, Appl. Phys. Lett. 98(11), 112106.

Asshoff, P., G. Wüst, A. Merz, D. Litvinov, D. Gerthsen, H. Kalt, and M. Hetterich, 2011b, Phys. Rev. B 84, 125302.

Atature, M., J. Dreiser, A. Badolato, A. Hogele, K. Karrai, and A. Imamoglu, 2006, Science 312(5773), 551.

Auer, T., R. Oulton, A. Bauschulte, D. R. Yakovlev, M. Bayer, S. Y. Verbin, R. V. Cherbunin, D. Reuter, and A. D. Wieck, 2009, Phys. Rev. B 80(20), 205303.

Balasubramanian, G., P. Neumann, D. Twitchen, R. Markham, M. Kolesov, N. Mizuochi, J. Isoya, J. Achard, J. Beck, J. Tissler, V. Jacques, P. R. Hemmer, F. Jelezko, et al., 2009, Nature Materials 8, 383.

Barnes, E., and S. E. Economou, 2011, Phys. Rev. Lett. 107, 047601.

Baugh, J., Y. Kitamura, K. Ono, and S. Tarucha, 2007, Phys. Rev. Lett. 99, 096804.

Bayer, M., G. Ortner, O. Stern, A. Kuther, A. A. Gorbunov, A. Forchel, P. Hawrylak, S. Fafard, K. Hinzer, T. L. Reinecke, S. N. Walck, J. P. Reithmaier, et al., 2002, Phys. Rev. B 65(19), 195315.

Belhadj, T., T. Amand, A. Kunold, C.-M. Simon, T. Kuroda, M. Abbarchi, T. Mano, K. Sakoda, S. Kunz, X. Marie, and B. Urbaszek, 2010, Applied Physics Letters 97(5), 051111 (pages 3).

Belhadj, T., T. Kuroda, C.-M. Simon, T. Amand, T. Mano, K. Sakoda, N. Koguchi, X. Marie, and B. Urbaszek, 2008, Phys. Rev. B 78(20), 205325.

Belhadj, T., C.-M. Simon, T. Amand, P. Renucci, B. Chatel, O. Krebs, A. Lemaître, P. Voisin, X. Marie, and B. Urbaszek, 2009, Phys. Rev. Lett. 103(8), 086601.

Besombes, L., K. Kheng, and D. Martrou, 2000, Phys. Rev. Lett. 85(2), 425.

Bester, G., D. Reuter, L. He, A. Zunger, P. Kailuweit, A. D. Wieck, U. Zeitler, J. C. Maan, O. Wibbelhoff, and A. Lorke, 2007, Phys. Rev. B 76, 075338.

Bester, G., and A. Zunger, 2005, Phys. Rev. B 71, 045318.

Bluhm, H., S. Foletti, I. Neder, M. Rudner, D. Mahalu, V. Umansky, and A. Yacoby, 2010, Nature Physics 7, 109.

Bracker, A. S., D. Gammon, and V. L. Korenev, 2008, Semiconductor Science and Technology 23(11), 114004.

Bracker, A. S., E. A. Stinaff, D. Gammon, M. E. Ware, J. G. Tischler, A. Shabaev, A. L. Efros, D. Park, D. Gershoni, V. L. Korenev, and I. A. Merkulov, 2005, Phys. Rev. Lett. 94(4), 047402.

Braun, P.-F., , B. Eble, L. Lombez, B. Urbaszek, T. Amand, X. Marie, P. Renucci, O. Krebs, A. Lemaitre, P. Voisin,
V. K. Kalevich, et al., 2006a, phys. stat. sol. (b) 243(15), 3917.

Braun, P.-F., X. Marie, L. Lombez, B. Urbaszek, T. Amand, P. Renucci, V. K. Kalevich, K. V. Kavokin, O. Krebs, P. Voisin, and Y. Masumoto, 2005, Phys. Rev. Lett. 94(11), 116601.

Braun, P.-F., B. Urbaszek, T. Amand, X. Marie, O. Krebs, B. Eble, A. Lemaitre, and P. Voisin, 2006b, Phys. Rev. B 74(24), 245306.

Brossel, J., A. Kastler, and J. Winter, 1952, J. de Physique et le Radium 13, 668.

Brown, S. W., T. A. Kennedy, and D. Gammon, 1998, Solid State Nuclear Magnetic Resonance 11(1-2), 49

Brunner, D., B. D. Gerardot, P. A. Dalgarno, G. Wost, K. Karrai, N. G. Stoltz, P. M. Petroff, and R. J. Warburton, 2009, Science 325(5936), 70.

Bulutay, C., 2012, Phys. Rev. B 85, 115313.

Burkard, G., D. Loss, and D. P. DiVincenzo, 1999, Phys. Rev. B 59(3), 2070.

Calarco, T., A. Datta, P. Fedichev, E. Pazy, and P. Zoller, 2003, Phys. Rev. A 68(1), 012310.

Chekhovich, E. A., A. B. Krysa, M. Hopkinson, P. Senellart, A. Lemaitre, M. S. Skolnick, and A. I. Tartakovskii, 2011, ArXiv eprint 1109.0733.

Chekhovich, E. A., A. B. Krysa, M. S. Skolnick, and A. I. Tartakovskii, 2011a, Phys. Rev. Lett. 106(2), 027402.

Chekhovich, E. A., A. B. Krysa, M. S. Skolnick, and A. I. Tartakovskii, 2011b, Phys. Rev. B 83(12), 125318.

Chekhovich, E. A., M. N. Makhonin, K. V. Kavokin, A. B. Krysa, M. S. Skolnick, and A. I. Tartakovskii, 2010a, Phys. Rev. Lett. 104(6), 066804.

Chekhovich, E. A., M. N. Makhonin, J. Skiba-Szymanska, A. B. Krysa, V. D. Kulakovskii, M. S. Skolnick, and A. I. Tartakovskii, 2010b, Phys. Rev. B 81(24), 245308.

Cherbunin, R. V., K. Flisinski, I. Y. Gerlovin, I. V. Ignatiev, M. S. Kuznetsova, M. Y. Petrov, D. R. Yakovlev, D. Reuter, A. D. Wieck, and M. Bayer, 2011, Phys. Rev. B 84(4), 041304.

Cherbunin, R. V., S. Y. Verbin, T. Auer, D. R. Yakovlev, D. Reuter, A. D. Wieck, I. Y. Gerlovin, I. V. Ignatiev, D. V. Vishnevsky, and M. Bayer, 2009, Phys. Rev. B 80(3), 035326.

Childress, L., M. V. Gurudev Dutt, J. M. Taylor, A. S. Zibrov, F. Jelezko, J. Wrachtrup, P. R. Hemmer, and M. D. Lukin, 2006, Science 314(5797), 281.

Colton, J. S., T. A. Kennedy, A. S. Bracker, and D. Gammon, 2004, Phys. Rev. B 69(12), 121307.

Cortez, S., O. Krebs, S. Laurent, M. Senes, X. Marie, P. Voisin, R. Ferreira, G. Bastard, J.-M. Gérard, and T. Amand, 2002, Phys. Rev. Lett. 89(20), 207401.

Crooker, S. A., J. Brandt, C. Sandfort, A. Greilich, D. R. Yakovlev, D. Reuter, A. D. Wieck, and M. Bayer, 2010, Phys. Rev. Lett. 104, 036601.

Cywinski, L., W. M. Witzel, and S. Das Sarma, 2009, Phys. Rev. Lett. 102(5), 057601.

Dahbashi, R., J. Hübner, F. Berski, J. Wiegand, X. Marie, K. Pierz, H. W. Schumacher, and M. Oestreich, 2012, Applied Physics Letters 100(3), 031906.

Damen, T. C., L. Via, J. E. Cunningham, J. Shah, and L. J. Sham, 1991, Phys. Rev. Lett. 67(24), 3432.

Desfonds, P., B. Eble, F. Fras, C. Testelin, F. Bernardot, M. Chamarro, B. Urbaszek, T. Amand, X. Marie, J. M. Gerard, V. Thierry-Mieg, A. Miard, et al., 2010, Appl. Phys. Lett. 96(17). 
Dou, X. M., B. Q. Sun, D. S. Jiang, H. Q. Ni, and Z. C. Niu, 2011, Phys. Rev. B 84(3), 033302.

Dousse, A., J. Suffczynski, A. Beveratos, O. Krebs, A. Lemaitre, I. Sagnes, J. Bloch, P. Voisin, and P. Senellart, 2010, Nature 466, 217.

Dreiser, J., M. Atatüre, C. Galland, T. Müller, A. Badolato, and A. Imamoglu, 2008, Phys. Rev. B 77(7), 075317.

Drexler, H., D. Leonard, W. Hansen, J. P. Kotthaus, and P. M. Petroff, 1994, Phys. Rev. Lett. 73(16), 2252.

Dutt, M. V. G., J. Cheng, B. Li, X. Xu, X. Li, P. R. Berman, D. G. Steel, A. S. Bracker, D. Gammon, S. E. Economou, R.-B. Liu, and L. J. Sham, 2005, Phys. Rev. Lett. 94(22), 227403.

Dyakonov, M., 2008, Springer Series in Solid-State Science, Springer-Verlag Berlin 157.

Dyakonov, M., and V. Perel., 1973, Zh.E ksp. Teor. Fiz. 65(362).

Dyakonov, M., and V. Perel., 1974, Sov. Phys. JETP 38(177).

Dzhioev, R. I., and V. L. Korenev, 2007, Phys. Rev. Lett. 99, 037401.

Dzhioev, R. I., V. L. Korenev, I. A. Merkulov, B. P. Zakharchenya, D. Gammon, A. L. Efros, and D. S. Katzer, 2002, Phys. Rev. Lett. 88(25), 256801.

Dzhioev, R. I., B. P. Zakharchenya, E. L. Ivchenko, V. L. Korenev, Y. G. Kusraev, N. N. Ledentsov, V. M. Ustinov, A. E. Zhukov, and A. F. Tsatsulnikov, 1998a, Phys. Sol. Sta. 40, 790.

Dzhioev, R. I., B. P. Zakharchenya, V. L. Korenev, P. E. Pak, D. A. Vinokurov, O. V. Kovalenkov, and I. S. Tarasov, 1998b, Physics of the Solid State 40(24), 1587.

Ebbens, A., D. N. Krizhanovskii, A. I. Tartakovskii, F. Pulizzi, T. Wright, A. V. Savelyev, M. S. Skolnick, and M. Hopkinson, 2005, Phys. Rev. B 72, 73307.

Eble, B., P. Desfonds, F. Bernardot, C. Testelin, and M. Chamarro, 2010, Phys. Rev. B 81(045322), 8.

Eble, B., O. Krebs, A. Lemaître, K. Kowalik, A. Kudelski, P. Voisin, B. Urbaszek, X. Marie, and T. Amand, 2006, Phys. Rev. B 74(8), 081306.

Eble, B., C. Testelin, P. Desfonds, F. Bernardot, A. Balocchi, T. Amand, A. Miard, A. Lemaître, X. Marie, and M. Chamarro, 2009, Phys. Rev. Lett. 102(14), 146601.

Elzerman, J. M., R. Hanson, L. H. W. van Beveren, B. Witkamp, L. M. K. Vandersypen, and L. P. Kouwenhoven, 2004, Nature 430, 431.

Erlingsson, S. I., Y. V. Nazarov, and V. I. Fal'ko, 2001, Phys. Rev. B 64(19), 195306.

Fallahi, P., S. T. Y ılmaz, and A. Imamoğlu, 2010, Phys. Rev. Lett. 105(25), 257402.

Feng, D. H., I. A. Akimov, and F. Henneberger, 2007, Phys. Rev. Lett. 99(3), 036604.

Fischer, J., W. A. Coish, D. V. Bulaev, and D. Loss, 2008, Phys. Rev. B 78(15), 155329.

Flisinski, K., I. Y. Gerlovin, I. V. Ignatiev, M. Y. Petrov, S. Y. Verbin, D. R. Yakovlev, D. Reuter, A. D. Wieck, and M. Bayer, 2010, Phys. Rev. B 82(8), 081308.

Flissikowski, T., I. A. Akimov, A. Hundt, and F. Henneberger, 2003, Phys. Rev. B 68(16), 161309.

Flissikowski, T., A. Hundt, M. Lowisch, M. Rabe, and F. Henneberger, 2001, Phys. Rev. Lett. 86(14), 3172.

Fras, F., B. Eble, P. Desfonds, F. Bernardot, C. Testelin, M. Chamarro, A. Miard, and A. Lemaitre, 2011, Phys. Rev. B 84, 125431.

Gammon, D., S. Brown, E. Snow, T. Kennedy, D. Katzer, and D. Park, 1997, Science 277, 85.
Gammon, D., A. L. Efros, T. A. Kennedy, M. Rosen, D. S. Katzer, D. Park, S. W. Brown, V. L. Korenev, and I. A. Merkulov, 2001, Phys. Rev. Lett. 86(22), 5176.

Gammon, D., E. S. Snow, B. V. Shanabrook, D. S. Katzer, and D. Park, 1996, Phys. Rev. Lett. 76(16), 3005.

Gerardot, B. D., D. Brunner, P. A. Dalgarno, P. Ohberg, S. Seidl, M. Kroner, K. Karrai, N. G. Stoltz, P. M. Petroff, and R. J. Warburton, 2008, Nature 451, 441.

Girard, J. C., A. Lemaître, A. Miard, C. David, and Z. Z. Wang, 2009 (AVS), volume 27, pp. 891-894.

Glazov, M. M., I. A. Yugova, and A. L. Efros, 2012, Phys. Rev. B 85, 041303.

Godden, T. M., J. H. Quilter, A. J. Ramsay, Y. Wu, P. Brereton, S. J. Boyle, I. J. Luxmoore, J. Puebla-Nunez, A. M. Fox, and M. S. Skolnick, 2012, Phys. Rev. Lett. 108, 017402.

Goldman, M., 1970, Spin Temperature and nuclear Magnetic Resonance in Solids (Oxford University Press).

Goldstein, L., F. Glas, J. Y. Marzin, M. N. Charasse, and G. L. Roux, 1985, Applied Physics Letters 47(10), 1099.

Greilich, A., S. G. Carter, D. Kim, A. S. Bracker, and D. Gammon, 2011, Nature Photonics 5, 702708.

Greilich, A., R. Oulton, E. A. Zhukov, I. A. Yugova, D. R. Yakovlev, M. Bayer, A. Shabaev, A. L. Efros, I. A. Merkulov, V. Stavarache, D. Reuter, and A. Wieck, 2006a, Phys. Rev. Lett. 96(22), 227401.

Greilich, A., A. Shabaev, D. R. Yakovlev, A. L. Efros, I. A. Yugova, D. Reuter, A. D. Wieck, and M. Bayer, 2007, Science 317(5846), 1896.

Greilich, A., D. R. Yakovlev, A. Shabaev, A. L. Efros, I. A. Yugova, R. Oulton, V. Stavarache, D. Reuter, A. Wieck, and M. Bayer, 2006b, Science 313(5785), 341.

Greve, K. D., P. L. McMahon, D. Press, T. D. L. D. Bisping, C. Schneider, M. Kamp, L. Worschech, S. Hfling, A. Forchel, and Y. Yamamoto, 2011, Nature Physics 7, 872878.

Grundmann, M., O. Stier, and D. Bimberg, 1995, Phys. Rev. B 52, 11969.

Gryncharova, E., and V. Perel, 1977, Sov. Phys. Semicond. 11(997).

Hanson, R., L. P. Kouwenhoven, J. R. Petta, S. Tarucha, and L. M. K. Vandersypen, 2007, Rev. Mod. Phys. 79(4), 1217.

Heiss, D., S. Schaeck, H. Huebl, M. Bichler, G. Abstreiter, J. J. Finley, D. V. Bulaev, and D. Loss, 2007, Phys. Rev. B 76, 241306.

Henneberger, F., and O. Benson, 2008, Pan Stanford Publishing .

Högele, A., M. Kroner, C. Latta, M. Claassen, I. Carusotto, C. Bulutay, and A. Imamoglu, 2012, Phys. Rev. Lett. 108, 197403.

Högele, A., S. Seidl, M. Kroner, K. Karrai, R. J. Warburton, B. D. Gerardot, and P. M. Petroff, 2004, Phys. Rev. Lett. 93(21), 217401.

Hours, J., P. Senellart, E. Peter, A. Cavanna, and J. Bloch, 2005, Phys. Rev. B 71(16), 161306.

Huang, C.-W., and X. Hu, 2010, Phys. Rev. B 81, 205304.

Imamoglu, A., D. D. Awschalom, G. Burkard, D. P. DiVincenzo, D. Loss, M. Sherwin, and A. Small, 1999, Phys. Rev. Lett. 83(20), 4204.

Imamoglu, A., E. Knill, L. Tian, and P. Zoller, 2003, Phys. Rev. Lett. 91(1), 017402.

Issler, M., E. M. Kessler, G. Giedke, S. Yelin, I. Cirac, M. D. Lukin, and A. Imamoglu, 2010, Phys. Rev. Lett. 105(26), 267202 . 
Kaji, R., S. Adachi, H. Sasakura, and S. Muto, 2008, Phys. Rev. B 77(11), 115345.

Kalevich, V. K., M. Paillard, K. V. Kavokin, X. Marie, A. R. Kovsh, T. Amand, A. E. Zhukov, Y. G. Musikhin, V. M. Ustinov, E. Vanelle, and B. P. Zakharchenya, 2001, Phys. Rev. B 64(4), 045309.

Karrai, K., R. J. Warburton, C. Schulhauser, A. Högele, B. Urbaszek, E. J. McGhee, A. O. Govorov, J. M. Garcia, B. D. Gerardot, and P. M. Petroff, 2004, Nature 427, 135.

Keizer, J. G., J. Bocquel, P. M. Koenraad, T. Mano, T. Noda, and K. Sakoda, 2010, Applied Physics Letters 96(6), 062101 (pages 3).

Kessler, E. M., G. Giedke, A. Imamoglu, S. F. Yelin, M. D. Lukin, and J. I. Cirac, 2012, ArXiv e-prints eprint 1205.3341.

Kessler, E. M., S. Yelin, M. D. Lukin, J. I. Cirac, and G. Giedke, 2010, Phys. Rev. Lett. 104(14), 143601.

Khaetskii, A., D. Loss, and L. Glazman, 2003, Phys. Rev. B 67, 195329.

Khaetskii, A. V., D. Loss, and L. Glazman, 2002, Phys. Rev. Lett. 88(18), 186802.

Khaetskii, A. V., and Y. V. Nazarov, 2000, Phys. Rev. B 61(19), 12639.

Klauser, D., W. A. Coish, and D. Loss, 2006, Phys. Rev. B 73, 205302.

Kloeffel, C., P. A. Dalgarno, B. Urbaszek, B. D. Gerardot, D. Brunner, P. M. Petroff, D. Loss, and R. J. Warburton, 2011, Phys. Rev. Lett. 106(4), 046802.

Klotz, F., V. Jovanov, J. Kierig, E. C. Clark, M. Bichler, G. Abstreiter, M. S. Brandt, J. J. Finley, H. Schwager, and G. Giedke, 2010, Phys. Rev. B 82(12), 121307.

Knight, W. D., 1949, Phys. Rev. 76(8), 1259.

Koguchi, N., S. Takahashi, and T. Chikyow, 1991, J. Cryst. Growth 111, 688.

Korenev, V. L., 2007, Phys. Rev. Lett. 99(25), 256405.

Koudinov, A. V., I. A. Akimov, Y. G. Kusrayev, and F. Henneberger, 2004, Phys. Rev. B 70(24), 241305.

Kozlov, G. G., 2007, JETP 105, 803.

Krebs, O., B. Eble, A. Lemaître, P. Voisin, B. Urbaszek, T. Amand, and X. Marie, 2008, C.R. Physique 9, 874.

Krebs, O., P. Maletinsky, T. Amand, B. Urbaszek, A. Lemaître, P. Voisin, X. Marie, and A. Imamoglu, 2010, Phys. Rev. Lett. 104(5), 056603.

Krebs, O., and P. Voisin, 1996, Phys. Rev. Lett. 77, 1829.

Krizhanovskii, D. N., A. Ebbens, A. I. Tartakovskii, F. Pulizzi, T. Wright, M. S. Skolnick, and M. Hopkinson, 2005, Phys. Rev. B 72(16), 161312.

Kroner, M., K. M. Weiss, B. Biedermann, S. Seidl, S. Manus, A. W. Holleitner, A. Badolato, P. M. Petroff, B. D. Gerardot, R. J. Warburton, and K. Karrai, 2008, Phys. Rev. Lett. 100(15), 156803.

Kroutvar, M., Y. Ducommun, D. Heiss, M. Bichler, D. Schuh, G. Abstreiter, and J. J. Finley, 2004, Nature 432, 81.

Lai, C. W., P. Maletinsky, A. Badolato, and A. Imamoglu, 2006, Phys. Rev. Lett. 96(16), 167403.

Lampel, G., 1968, Phys. Rev. Lett. 20(10), 491.

Larsson, L. A., E. S. Moskalenko, and P. O. Holtz, 2011, Applied Physics Letters 98(7), 071906 (pages 3).

Latta, C., F. Haupt, M. Hanl, A. Weichselbaum, M. Claassen, W. Wuester, P. Fallahi, S. Faelt, L. Glazman, J. von Delft, H. E. Tureci, and A. Imamoglu, 2011a, Nature 474, 627.

Latta, C., A. Hogele, Y. Zhao, A. N. Vamivakas, P. Maletinsky, M. Kroner, J. Dreiser, I. Carusotto,
A. Badolato, D. Schuh, W. Wegscheider, M. Atature, et al., 2009, Nature Phys. 5(8), 758.

Latta, C., A. Srivastava, and A. Imamoğlu, 2011b, Phys. Rev. Lett. 107, 167401.

Laurent, S., B. Eble, O. Krebs, A. Lemaître, B. Urbaszek, X. Marie, T. Amand, and P. Voisin, 2005, Phys. Rev. Lett. 94(14), 147401.

Laurent, S., M. Senes, O. Krebs, V. K. Kalevich, B. Urbaszek, X. Marie, T. Amand, and P. Voisin, 2006, Phys. Rev. B 73(23), 235302.

Léger, Y., L. Besombes, L. Maingault, and H. Mariette, 2007, Phys. Rev. B 76(4), 045331.

Leonard, D., K. Pond, and P. M. Petroff, 1994, Phys. Rev. B $\mathbf{5 0}(16), 11687$.

Li, C. H., G. Kioseoglou, O. M. J. van Erve, M. E. Ware, D. Gammon, R. M. Stroud, B. T. Jonker, R. Mallory, M. Yasar, and A. Petrou, 2005, Appl. Phys. Lett. 86(13), 132503.

Li, Y., N. Sinitsyn, D. L. Smith, D. Reuter, A. D. Wieck, D. R. Yakovlev, M. Bayer, and S. A. Crooker, 2012, Phys. Rev. Lett. 108, 186603.

Liu, W. K., K. M. Whitaker, A. L. Smith, K. R. Kittilstved, B. H. Robinson, and D. R. Gamelin, 2007, Phys. Rev. Lett. 98(18), 186804.

Lombez, L., P.-F. Braun, X. Marie, P. Renucci, B. Urbaszek, T. Amand, O. Krebs, and P. Voisin, 2007a, Phys. Rev. B 75(19), 195314.

Lombez, L., P. Renucci, P. F. Braun, H. Carrere, X. Marie, T. Amand, B. Urbaszek, J. L. Gauffier, P. Gallo, T. Camps, A. Arnoult, C. Fontaine, et al., 2007b, Appl. Phys. Lett. $\mathbf{9 0}(8), 081111$.

Lu, C.-Y., Y. Zhao, A. N. Vamivakas, C. Matthiesen, S. Fält, A. Badolato, and M. Atatüre, 2010, Phys. Rev. B 81(3), 035332 .

Lu, J., M. J. R. Hoch, P. L. Kuhns, W. G. Moulton, Z. Gan, and A. P. Reyes, 2006, Phys. Rev. B 74(12), 125208.

Makhonin, M. N., E. A. Chekhovich, P. Senellart, A. Lemaître, M. S. Skolnick, and A. I. Tartakovskii, 2010, Phys. Rev. B 82(16), 161309.

Maletinsky, P., 2008, Polarization and Manipulation of a Mesoscopic Nuclear Spin Ensemble Using a Single Confined Electron Spin, Ph.D. thesis, ETH Zürich, URL http: //e-collection.ethbib.ethz.ch/view/eth:30788

Maletinsky, P., A. Badolato, and A. Imamoglu, 2007a, Phys. Rev. Lett. 99(5), 056804.

Maletinsky, P., M. Kroner, and A. Imamoglu, 2009, Nature Phys. 5(6), 407.

Maletinsky, P., C. W. Lai, A. Badolato, and A. Imamoglu, 2007b, Phys. Rev. B 75(3), 035409.

Malinowski, A., M. A. Brand, and R. T. Harley, 2001, Physica E 10, 13 .

Marzin, J. Y., J. M. Gérard, A. Izraël, D. Barrier, and G. Bastard, 1994, Phys. Rev. Lett. 73(5), 716.

McNeil, J. A., and W. G. Clark, 1976, Phys. Rev. B 13, 4705.

Meier, F., and B. Zakharchenya, 1984, Modern Problems in Condensed Matter Sciences (North-Holland, Amsterdam). 8.

Merkulov, I. A., A. L. Efros, and M. Rosen, 2002, Phys. Rev. B 65(20), 205309.

Michler, P., A. Kiraz, C. Becher, W. V. Schoenfeld, P. M. Petroff, L. Zhang, E. Hu, and A. Imamoglu, 2000, Science 290(5500), 2282.

Moskalenko, E. S., L. A. Larsson, and P. O. Holtz, 2009, Phys. Rev. B 80(19), 193413. 
Muller, A., E. B. Flagg, P. Bianucci, X. Y. Wang, D. G. Deppe, W. Ma, J. Zhang, G. J. Salamo, M. Xiao, and C. K. Shih, 2007, Phys. Rev. Lett. 99(18), 187402.

Nikolaenko, A. E., E. A. Chekhovich, M. N. Makhonin, I. W. Drouzas, A. B. Van'kov, J. Skiba-Szymanska, M. S. Skolnick, P. Senellart, D. Martrou, A. Lemaître, and A. I. Tartakovskii, 2009, Phys. Rev. B 79(8), 081303.

Offermans, P., P. Koenraad, J. Wolter, K. Pierz, M. Roy, and P. Maksym, 2005, Physica E 26(1-4), 236.

Oulton, R., A. Greilich, S. Y. Verbin, R. V. Cherbunin, T. Auer, D. R. Yakovlev, M. Bayer, I. A. Merkulov, V. Stavarache, D. Reuter, and A. D. Wieck, 2007, Phys. Rev. Lett. 98(10), 107401.

Overhauser, A. W., 1953, Phys. Rev. 92(2), 411.

Paget, D., 1982, Phys. Rev. B 25, 4444.

Paget, D., T. Amand, and J.-P. Korb, 2008, Phys. Rev. B 77(24), 245201.

Paget, D., G. Lampel, B. Sapoval, and V. I. Safarov, 1977, Phys. Rev. B 15, 5780.

Paillard, M., X. Marie, P. Renucci, T. Amand, A. Jbeli, and J. M. Gérard, 2001, Phys. Rev. Lett. 86(8), 1634.

Paillard, M., X. Marie, E. Vanelle, T. Amand, V. K. Kalevich, A. R. Kovsh, A. E. Zhukov, and V. M. Ustinov, 2000, Applied Physics Letters 76(1), 76.

Pal, B., S. Y. Verbin, I. V. Ignatiev, M. Ikezawa, and Y. Masumoto, 2007, Phys. Rev. B 75(12), 125322.

Petrov, M. Y., G. G. Kozlov, I. V. Ignatiev, R. V. Cherbunin, D. R. Yakovlev, and M. Bayer, 2009, Phys. Rev. B 80, 125318.

Petta, J. R., A. C. Johnson, J. M. Taylor, E. A. Laird, A. Yacoby, M. D. Lukin, C. M. Marcus, M. P. Hanson, and A. C. Gossard, 2005, Science 309(5744), 2180.

Pines, D., J. Bardeen, and C. P. Slichter, 1957, Phys. Rev. 106, 489.

Press, D., K. De Greve, P. L. McMahon, T. D. Ladd, B. Friess, C. Schneider, M. Kamp, S. Hoefling, A. Forchel, and Y. Yamamoto, 2010, Nature Photonics 4, 367.

Rudner, M. S., L. M. K. Vandersypen, V. Vuletić, and L. S. Levitov, 2011, Phys. Rev. Lett. 107, 206806.

Sallen, G., B. Urbaszek, M. M. Glazov, E. L. Ivchenko, T. Kuroda, T. Mano, S. Kunz, M. Abbarchi, K. Sakoda, D. Lagarde, A. Balocchi, X. Marie, et al., 2011, Phys. Rev. Lett. 107, 166604.

Scheibner, M., G. Bacher, S. Weber, A. Forchel, T. Passow, and D. Hommel, 2003, Phys. Rev. B 67(15), 153302.

Schrieffer, J. R., and P. A. Wolff, 1966, Phys. Rev. 149, 491.

Schulhauser, C., 2004, Elektronische Quantenpunktzustände induziert durch Photoemission, Ph.D. thesis, LMU München, URL http://edoc.ub.uni-muenchen.de/ 2685/

Schulten, K., and P. G. Wolynes, 1978, J. Chem. Phys. 68, 3292.

Semenov, Y. G., and K. W. Kim, 2003, Phys. Rev. B 67(7), 073301.

Sénès, M., X. Marie, B. Urbaszek, P. Renucci, T. Amand, and J.-M. Gérard, 2004, phys. stat. sol.(c) 1, 594.

Sénès, M., B. Urbaszek, X. Marie, T. Amand, J. Tribollet, F. Bernardot, C. Testelin, M. Chamarro, and J.-M. Gérard, 2005, Phys. Rev. B 71(11), 115334.

Shabaev, A., E. A. Stinaff, A. S. Bracker, D. Gammon, A. L. Efros, V. L. Korenev, and I. Merkulov, 2009, Phys. Rev. B 79(3), 035322.

Simon, C.-M., T. Belhadj, B. Chatel, T. Amand, P. Renucci, A. Lemaitre, O. Krebs, P. A. Dalgarno, R. J. Warburton,
X. Marie, and B. Urbaszek, 2011, Phys. Rev. Lett. 106, 166801.

Skiba-Szymanska, J., E. A. Chekhovich, A. E. Nikolaenko, A. I. Tartakovskii, M. N. Makhonin, I. Drouzas, M. S. Skolnick, and A. B. Krysa, 2008, Phys. Rev. B 77(16), 165338. Slichter, C. P., 1990, Springer-Verlag .

Smith, J. M., P. A. Dalgarno, R. J. Warburton, A. O. Govorov, K. Karrai, B. D. Gerardot, and P. M. Petroff, 2005, Phys. Rev. Lett. 94, 197402.

Stevenson, R. M., C. L. Salter, A. Boyer de la Giroday, I. A. Farrer, C. A. Nicoll, D. A. Ritchie, and A. J. Shields, 2011, ArXiv e-prints eprint 1103.2969.

Takahashi, R., K. Kono, S. Tarucha, and K. Ono, 2011, Phys. Rev. Lett. 107(2), 026602.

Tartakovskii, A. I., T. Wright, A. Russell, V. I. Fal'ko, A. B. Van'kov, J. Skiba-Szymanska, I. Drouzas, R. S. Kolodka, M. S. Skolnick, P. W. Fry, A. Tahraoui, H.-Y. Liu, et al., 2007, Phys. Rev. Lett. 98(2), 026806.

Taylor, J. M., C. M. Marcus, and M. D. Lukin, 2003, Phys. Rev. Lett. 90(20), 206803.

Testelin, C., F. Bernardot, B. Eble, and M. Chamarro, 2009, Phys. Rev. B 79(19), 195440.

Tong, H., and M. W. Wu, 2011, Phys. Rev. B 83, 235323.

Urbaszek, B., P.-F. Braun, T. Amand, O. Krebs, T. Belhadj, A. Lemaítre, P. Voisin, and X. Marie, 2007, Phys. Rev. B 76(20), 201301.

Urbaszek, B., R. J. Warburton, K. Karrai, B. D. Gerardot, P. M. Petroff, and J. M. Garcia, 2003, Phys. Rev. Lett. 90, 247403.

Vamivakas, N. A., Y. Zhao, C.-Y. Lu, and M. Atatüre, 2009, Nature Phys. 5, 198.

Verzelen, O., G. Bastard, and R. Ferreira, 2002, Phys. Rev. B 66, 081308.

Vink, I. T., K. C. Nowack, F. H. L. Koppens, J. Danon, Y. V. Nazarov, and L. M. K. Vandersypen, 2009, Nature Phys. 5, 764 .

Warburton, R. J., B. T. Miller, C. S. Dürr, C. Bödefeld, K. Karrai, J. P. Kotthaus, G. Medeiros-Ribeiro, P. M. Petroff, and S. Huant, 1998, Phys. Rev. B 58, 16221.

Warburton, R. J., C. Schäflein, D. Haft, F. Bickel, A. Lorke, K.Karrai, J. Garcia, W. Schoenfeld, and P. M. Petroff, 2000, Nature (London) 405, 926.

Ware, M. E., E. A. Stinaff, D. Gammon, M. F. Doty, A. S. Bracker, D. Gershoni, V. L. Korenev, i. m. c. C. Bădescu, Y. Lyanda-Geller, and T. L. Reinecke, 2005, Phys. Rev. Lett. 95, 177403.

Whitaker, K. M., S. T. Ochsenbein, A. L. Smith, D. C. Echodu, B. H. Robinson, and D. R. Gamelin, 2010, J. Phys. Chem. C 114(34), 14467.

Williams, W., 1991, Clarendon Press, Oxford .

Witzel, W. M., and S. Das Sarma, 2007, Phys. Rev. B 76(4), 045218.

Xu, X., Y. Wu, B. Sun, Q. Huang, J. Cheng, D. G. Steel, A. S. Bracker, D. Gammon, C. Emary, and L. J. Sham, 2007, Phys. Rev. Lett. 99, 097401.

Xu, X., W. Yao, B. Sun, D. G. Steel, A. S. Bracker, D. Gammon, and L. J. Sham, 2009, Nature (London) 459, 1105.

Yang, W., and L. J. Sham, 2010, ArXiv e-prints eprint 1012.0060.

Yao, W., R.-B. Liu, and L. J. Sham, 2006, Phys. Rev. B 74, 195301.

Yilmaz, S. T., P. Fallahi, and A. Imamoglu, 2010, Phys. Rev. Lett. 105(3), 033601.

Yugova, I. A., A. Greilich, E. A. Zhukov, D. R. Yakovlev, 
M. Bayer, D. Reuter, and A. D. Wieck, 2007, Phys. Rev. B 75(19), 195325.

Zhang, W., V. V. Dobrovitski, K. A. Al-Hassanieh,
E. Dagotto, and B. N. Harmon, 2006, Phys. Rev. B 74 205313. 
TABLE IV Definitions

\begin{tabular}{|c|c|}
\hline symbol & meaning \\
\hline$N$ & number of nuclei in the dot \\
\hline$\Gamma$ & spontaneous emission rate \\
\hline$\tau_{r}=1 / \Gamma$ & radiative recombination time \\
\hline$\hbar \omega_{Z}^{e}$ & electron Zeeman splitting \\
\hline$\hbar \omega_{Z}^{h}$ & hole Zeeman splitting \\
\hline$\hbar \omega_{Z}^{X}$ & exciton Zeeman splitting \\
\hline$\hbar \omega_{Z}^{n}$ & nuclear Zeeman splitting \\
\hline$\hbar \omega_{O S}^{e}$ & electron Overhauser splitting \\
\hline$\hbar \omega_{O S}^{h}$ & hole Overhauser splitting \\
\hline$B_{K}$ & Knight field \\
\hline $\boldsymbol{B}_{n}=\left(B_{n, x}, B_{n, y}, B_{n, z}\right)$ & Overhauser field \\
\hline$\delta B_{n}$ & Nuclear Field Fluctuations \\
\hline $\boldsymbol{B}=\left(B_{x}, B_{y}, B_{z}\right)$ & external magnetic field \\
\hline$B_{\text {tot }}$ & total magnetic field experienced by electron \\
\hline$B_{\text {tot }}^{h}$ & total magnetic field experienced by hole \\
\hline$\underset{\sim}{A_{j}}$ & hyperfine const. for nuclear species $j$ coupling to electrons \\
\hline$\tilde{A}$ & effective hyperfine constant for coupling to electrons \\
\hline$A_{j}^{h}$ & hyperfine constant for nuclear species $j$ coupling to holes \\
\hline$\tilde{A}^{h}$ & effective hyperfine constant for coupling to holes \\
\hline$A_{i}^{\mathrm{nc}}$ & non-collinear hyperfine coupling of $i$-th nucleus \\
\hline$\uparrow$ or $\downarrow$ & electron spin state in " $z$ " basis \\
\hline$\Uparrow$ or $\Downarrow$ & hole pseudo-spin state in " $z$ " basis \\
\hline write $\sum_{j} I_{j, z}$ as $\left|I_{z}\right\rangle$ & nuclear spin $z$ projection \\
\hline$S_{z}$ & electron spin $z$ projection \\
\hline$S_{z}^{h}$ & hole 'pseudo' spin $z$ projection \\
\hline$J$ & hole angular momentum \\
\hline$J_{z}$ & hole angular momentum $z$ projection \\
\hline$T_{\Delta}$ & $\begin{array}{l}\text { electron spin dephasing time in randomly distributed frozen fluctuation of the hyper- } \\
\text { fine field }\end{array}$ \\
\hline$T_{\text {Dipole }}$ & average precession time of a nuclear spin in the local field fluctuations $\delta B_{L}$ \\
\hline$T_{1 \mathrm{e}}$ & electron-induced of nuclear spins \\
\hline$T_{d}$ & decay time of average nuclear spin \\
\hline$T_{1 \mathrm{~d}}$ & spin flip time experienced by electron due to flip-flops with nuclei \\
\hline$\tau_{c}^{e}$ & electron correlation time \\
\hline$\tau_{c}^{h}$ & hole correlation time \\
\hline$\tau_{c}^{n}$ & correlation time of nuclei \\
\hline$\tau_{s}^{e}$ & electron spin relaxation time \\
\hline$\tau_{s}^{h}$ & hole spin relaxation time \\
\hline$g_{z}^{e}$ & longitudinal electron g-factor \\
\hline$g_{z}^{h}$ & longitudinal hole electron g-factor \\
\hline$g_{\perp}^{e}$ & transverse electron g-factor \\
\hline$g_{\perp}^{\bar{h}}$ & transverse hole g-factor \\
\hline DNP & Dynamic Nuclear (Spin) Polarization \\
\hline$X^{0}$ & neutral exciton \\
\hline$X^{-}$ & negatively charged exciton \\
\hline$X^{+}$ & positively charged exciton \\
\hline$\delta_{1}$ & fine structure splitting of $\mathrm{J}=1 X^{0}$ due to anisotropic e-h Coulomb exchange interaction \\
\hline$\delta_{2}$ & fine structure splitting of $\mathrm{J}=2 X^{0}$ due to anisotropic e-h Coulomb exchange interaction \\
\hline$\delta_{0}$ & $\begin{array}{l}\text { fine structure splitting of between } \mathrm{J}=2 \text { and } \mathrm{J}=1 X^{0} \text { due to isotropic e-h Coulomb } \\
\text { exchange interaction }\end{array}$ \\
\hline$\kappa$ & electron co-tunelling rate \\
\hline$\Delta \nu$ & QD absorption linewidth \\
\hline$\omega_{\mathrm{X}}$ & QD exciton transition frequency \\
\hline$\omega_{\mathrm{L}}$ & laser frequency \\
\hline$\Omega_{\mathrm{L}}$ & Rabi frequency \\
\hline$\Delta \omega=\omega_{\mathrm{X}}-\omega_{\mathrm{L}}$ & laser detuning from QD resonance \\
\hline$\omega_{\mathrm{X}}$ & QD exciton transition frequency \\
\hline
\end{tabular}

